Supporting Information For:

\title{
Catalytic Hydroxylcyclopropanol Ring-Opening Carbonylative Lactonization to Fused Bicyclic Lactones
}

\author{
Xinpei Cai, Weida Liang, Mingxin Liu, Xiating Li, and Mingji Dai* \\ Department of Chemistry and Center for Cancer Research \\ Purdue University, West Lafayette, IN 47907 (USA)
}




\section{Table of Contents}

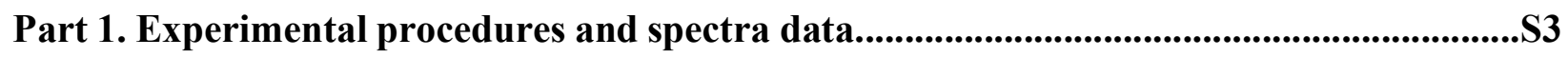

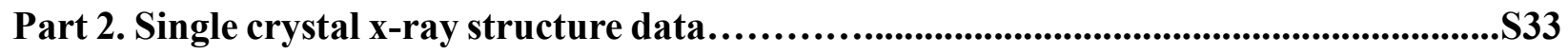

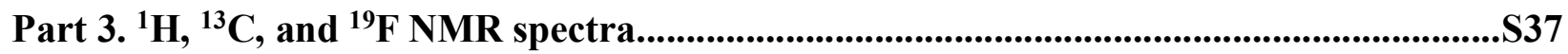




\section{Part 1. Experimental procedures and spectra data.}

General Methods: Reactions were performed using standard syringe techniques under argon unless stated otherwise. Starting materials and reagents were used as received from suppliers. Acetonitrile $\left(\mathrm{CH}_{3} \mathrm{CN}\right)$, dichloromethane $\left(\mathrm{CH}_{2} \mathrm{Cl}_{2}\right)$, methanol $(\mathrm{MeOH})$, tetrahydrofuran (THF), and toluene were purified by passing the previously degassed solvents through activated alumina columns. 1,2-Dichloroethane (DCE) was purified by distillation over calcium hydride. Benzoquinone was purified by recrystallization from hexanes. Flash chromatography was performed using silica gel (230-400 mesh). Thin layer chromatography (TLC) was performed using glass-backed silica plates (Silicycle). NMR spectra were recorded on a Bruker ARX-400 spectrometer or AV-500 spectrometer at room temperature. Chemical shifts (in ppm) are given in reference to the solvent signal $\left[{ }^{1} \mathrm{H}\right.$ NMR: $\mathrm{CDCl}_{3}$ (7.26); ${ }^{13} \mathrm{C} \mathrm{NMR} \mathrm{CDCl}_{3}$ (77.2)]. $1 \mathrm{H}$ NMR data are reported as follows: chemical shifts $(\delta \mathrm{ppm})$, multiplicity $(\mathrm{s}=$ singlet, $\mathrm{d}=$ doublet, $\mathrm{t}=$ triplet, $\mathrm{q}=$ quartet, quin $=$ quintuplet, $\mathrm{m}=$ multiplet, $\mathrm{br}=$ broad $)$, coupling constant $(\mathrm{Hz})$, and integration. ${ }^{13} \mathrm{C}$ NMR data are reported in terms of chemical shift and multiplicity. High-resolution mass measurements for compound characterization were carried out using a Waters SYNAPT G2-Si system with QuanTof analyzer or an Agilent 6550 QTOF system. IR data were recorded on a Thermo Nicolet Nexus 470 FTIR.

\section{General procedure for the synthesis of cyclopropanols: ${ }^{1}$}

A solution of titanium(IV) isopropoxide (1.1 equiv.) and the allyl alcohol (1.0 equiv.) in toluene (0.5 M) was stirred at room temperature for $1 \mathrm{~h}$, then at $40^{\circ} \mathrm{C}$ using an oil bath for $10 \mathrm{~min}$. After volatile components were removed under vacuum, THF $(0.1 \mathrm{M})$ and the corresponding ester (1.0 equiv.) were added at room temperature, followed by a solution of cyclopentylmagnesium chloride ( $2 \mathrm{M}, 4.4$ equiv.) in tetrahydrofuran, over a period of $1 \mathrm{~h}$ (with a syringe pump). The reaction mixture was then stirred for an additional $30 \mathrm{~min}$ before it was quenched by addition of water $(1 \mathrm{ml} / 1 \mathrm{mmol}$ alcohol $)$. The resulting mixture was stirred for 1 $\mathrm{h}$, dried over anhydrous sodium sulfate, and filtered. The filter cake was washed with $\mathrm{CH}_{2} \mathrm{Cl}_{2}$. The combined filtrates were concentrated under reduced pressure. The resulting residue was purified by flash chromatography.

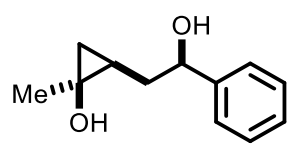

15a: $692 \mathrm{mg}$; 72\%; pale yellow oil; purified by column chromatography (hexane/ $\mathrm{Et}_{2} \mathrm{O}=2 / 1$ ). ${ }^{1} \mathbf{H}$ NMR $\left(500 \mathrm{MHz}, \mathrm{CDCl}_{3}\right) \delta 7.40-7.34(\mathrm{~m}, 4 \mathrm{H}), 7.28-7.25(\mathrm{~m}, 1 \mathrm{H}), 4.91(\mathrm{dd}, J=5.2,4.1 \mathrm{~Hz}, 1 \mathrm{H}), 2.92(\mathrm{~s}, 2 \mathrm{H})$, $2.25-2.22(\mathrm{~m}, 1 \mathrm{H}), 1.87-1.81(\mathrm{~m}, 1 \mathrm{H}), 1.30(\mathrm{~s}, 3 \mathrm{H}), 0.56-0.52(\mathrm{~m}, 1 \mathrm{H}), 0.42-0.38(\mathrm{~m}, 2 \mathrm{H}) .{ }^{13} \mathbf{C} \mathbf{~ N M R}$ $\left(125 \mathrm{MHz}, \mathrm{CDCl}_{3}\right) \delta 144.2,128.3,127.4,125.9,73.6,54.5,37.5,25.8,20.3,20.0$. IR $\left(\right.$ neat, $\left.\mathrm{cm}^{-1}\right): v=3308$, 
2952, 2916, 1451, 1377, 1270, 1045, 758, 699. HRMS (ESI): $m / z 175.1117$ calc. for $\mathrm{C}_{12} \mathrm{H}_{15} \mathrm{O}^{+}[\mathrm{M}+\mathrm{H}-$ $\left.\mathrm{H}_{2} \mathrm{O}\right]^{+}$, found 175.1119 .

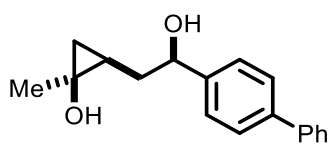

15b: $130 \mathrm{mg}$; 27\% yield; white solid; purified by column chromatography (hexane/ $\mathrm{Et}_{2} \mathrm{O}=2 / 1$ ). ${ }^{1} \mathbf{H}$ NMR $\left(500 \mathrm{MHz}, \mathrm{CDCl}_{3}\right) \delta 7.67-7.55(\mathrm{~m}, 4 \mathrm{H}), 7.47-7.40(\mathrm{~m}, 4 \mathrm{H}), 7.39-7.31(\mathrm{~m}, 1 \mathrm{H}), 5.00(\mathrm{t}, J=4.6 \mathrm{~Hz}$, $1 \mathrm{H}), 2.28(\mathrm{dt}, J=14.6,4.1 \mathrm{~Hz}, 1 \mathrm{H}), 1.92(\mathrm{ddd}, J=14.5,10.0,5.4 \mathrm{~Hz}, 1 \mathrm{H}), 1.36$ (s, 3H), 0.59 (dd, $J=9.0$, $4.7 \mathrm{~Hz}, 1 \mathrm{H}), 0.54-0.39$ (m, 2H). ${ }^{13} \mathbf{C}$ NMR $\left(125 \mathrm{MHz}, \mathrm{CDCl}_{3}\right) \delta 143.1,140.8,140.2,128.8,127.3,127.1$, 127.0, 126.2, 73.4, 54.5, 37.3, 25.7, 20.2, 20.0. IR (neat, $\mathrm{cm}^{-1}$ ): $v=3316,3028,2953,2916,1601,1488$, 1446, 1406, 1376, 1269, 1049, 1008, 971, 842, 765, 737, 697. HRMS (ESI): $\mathrm{m} / \mathrm{z} 291.1356$ calc. for $\mathrm{C}_{18} \mathrm{H}_{20} \mathrm{NaO}_{2}^{+}[\mathrm{M}+\mathrm{Na}]^{+}$, found 291.1355.

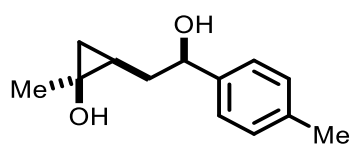

15c: $150 \mathrm{mg}$; 39\%; colorless liquid; purified by column chromatography (hexane/ $\mathrm{Et}_{2} \mathrm{O}=2 / 1$ ). ${ }^{1} \mathbf{H}$ NMR $\left(500 \mathrm{MHz}, \mathrm{CDCl}_{3}\right) \delta 7.25(\mathrm{~d}, J=8.0 \mathrm{~Hz}, 2 \mathrm{H}), 7.18-7.13(\mathrm{~m}, 2 \mathrm{H}), 4.90-4.81(\mathrm{~m}, 1 \mathrm{H}), 3.08(\mathrm{~s}, 2 \mathrm{H}), 2.35$ (s, 3H), $2.20(\mathrm{dt}, J=14.4,4.0 \mathrm{~Hz}, 1 \mathrm{H}), 1.86-1.78(\mathrm{~m}, 1 \mathrm{H}), 1.30$ (s, 3H), 0.53 (q, $J=4.3 \mathrm{~Hz}, 1 \mathrm{H}), 0.46-$ 0.35 (m, 2H). ${ }^{13} \mathbf{C}$ NMR $\left(125 \mathrm{MHz}, \mathrm{CDCl}_{3}\right) \delta 141.0,136.9,128.9,125.7,73.4,54.3,37.4,25.7,21.1,20.3$, 19.8. IR (neat, $\mathrm{cm}^{-1}$ ): $v=3358,2922,1717,1445,1409,1376,1270,1179,1047,1020,972,815$. HRMS (ESI): $m / z 189.1274$ calc. for $\mathrm{C}_{13} \mathrm{H}_{17} \mathrm{O}^{+}\left[\mathrm{M}+\mathrm{H}-\mathrm{H}_{2} \mathrm{O}\right]^{+}$, found 189.1275 .

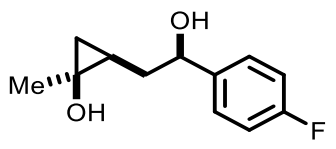

15d: $240 \mathrm{mg}$; 51\%; colorless liquid; purified by column chromatography (hexane/ $\mathrm{Et}_{2} \mathrm{O}=2 / 1$ ). ${ }^{1} \mathbf{H}$ NMR $\left(500 \mathrm{MHz}, \mathrm{CDCl}_{3}\right) \delta 7.34-7.26(\mathrm{~m}, 2 \mathrm{H}), 7.06-6.88(\mathrm{~m}, 2 \mathrm{H}), 4.87(\mathrm{t}, J=4.5 \mathrm{~Hz}, 1 \mathrm{H}), 3.80-3.68(\mathrm{~m}$, $1 \mathrm{H}), 3.68-3.53(\mathrm{~m}, 1 \mathrm{H}), 2.16$ (dt, $J=14.4,4.1 \mathrm{~Hz}, 1 \mathrm{H}), 1.80$ (ddd, $J=14.6,9.9,5.0 \mathrm{~Hz}, 1 \mathrm{H}), 1.30$ (s, 3H), $0.53(\mathrm{dd}, J=9.1,4.9 \mathrm{~Hz}, 1 \mathrm{H}), 0.43-0.24(\mathrm{~m}, 2 \mathrm{H}) .{ }^{13} \mathbf{C} \mathbf{N M R}\left(125 \mathrm{MHz}, \mathrm{CDCl}_{3}\right) \delta 161.9(\mathrm{~d}, J=274 \mathrm{~Hz})$, $139.8(\mathrm{~d}, J=3 \mathrm{~Hz}), 127.2(\mathrm{~d}, J=9 \mathrm{~Hz}), 114.9(\mathrm{~d}, J=21 \mathrm{~Hz}), 72.5,54.3,37.4,25.6,20.0,19.9 .{ }^{19} \mathbf{F}$ NMR $\left(471 \mathrm{MHz}, \mathrm{CDCl}_{3}\right) \delta-116.8$. IR (neat, $\left.\mathrm{cm}^{-1}\right): v=3330,2951,2868,1603,1508,1270,1220,1156,1049$, 1014, 835. HRMS (ESI): $m / z$ 193.1023 calc. for $\mathrm{C}_{12} \mathrm{H}_{14} \mathrm{FO}^{+}\left[\mathrm{M}+\mathrm{H}-\mathrm{H}_{2} \mathrm{O}\right]^{+}$, found 193.1025 . 


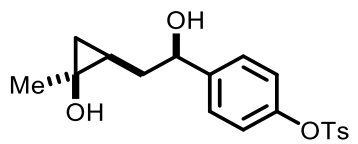

15e: $430 \mathrm{mg}$; 29\%; colorless solid; purified by column chromatography (hexane $\left./ \mathrm{Et}_{2} \mathrm{O}=2 / 1\right) .{ }^{1} \mathbf{H}$ NMR (500 $\left.\mathrm{MHz}, \mathrm{CDCl}_{3}\right) \delta 7.73-7.54(\mathrm{~m}, 2 \mathrm{H}), 7.34-7.23(\mathrm{~m}, 4 \mathrm{H}), 7.02-6.91(\mathrm{~m}, 2 \mathrm{H}), 4.89(\mathrm{t}, J=4.5 \mathrm{~Hz}, 1 \mathrm{H})$, 3.25 (s, 1H), 2.94 (s, 1H), 2.44 (s, 3H), 2.17 (dt, $J=14.6,4.2 \mathrm{~Hz}, 1 \mathrm{H}), 1.81$ (ddd, $J=14.9,10.3,5.0 \mathrm{~Hz}$, $1 \mathrm{H}), 1.29(\mathrm{~s}, 3 \mathrm{H}), 0.55(\mathrm{dd}, J=9.3,5.2 \mathrm{~Hz}, 1 \mathrm{H}), 0.38(\mathrm{t}, J=5.8 \mathrm{~Hz}, 1 \mathrm{H}), 0.35-0.24(\mathrm{~m}, 1 \mathrm{H}) .{ }^{13} \mathbf{C}$ NMR $\left(125 \mathrm{MHz}, \mathrm{CDCl}_{3}\right) \delta 148.5,145.4,143.2,132.4,129.8,128.5,126.9,122.1,72.6,54.4,37.3,25.6,21.7$, 20.0, 19.9. IR (neat, $\mathrm{cm}^{-1}$ ): $v=3324,2922,1597,1500,1370,1197,1174,1152,1093,865,815,663,552$.

HRMS (ESI): $m / z 385.1080$ calc. for $\mathrm{C}_{19} \mathrm{H}_{22} \mathrm{NaO}_{5} \mathrm{~S}^{+}[\mathrm{M}+\mathrm{Na}]^{+}$, found 385.1080 .

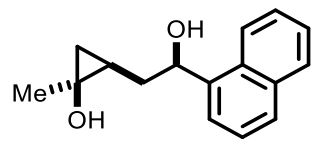

15f: $480 \mathrm{mg}$; 31\%; white solid; purified by column chromatography (hexane/ $\left.\mathrm{Et}_{2} \mathrm{O}=2 / 1\right) ;{ }^{1} \mathbf{H}$ NMR (500 $\left.\mathrm{MHz}, \mathrm{CDCl}_{3}\right) \delta 8.05(\mathrm{~d}, J=8.1 \mathrm{~Hz}, 1 \mathrm{H}), 7.89(\mathrm{dd}, J=7.6,2.1 \mathrm{~Hz}, 1 \mathrm{H}), 7.77(\mathrm{dd}, J=20.1,7.6 \mathrm{~Hz}, 2 \mathrm{H})$, $7.51(\mathrm{ddd}, J=9.7,5.4,2.9 \mathrm{~Hz}, 3 \mathrm{H}), 5.77$ (t, $J=4.5 \mathrm{~Hz}, 1 \mathrm{H}), 3.00(\mathrm{~s}, 1 \mathrm{H}), 2.77$ (s, 1H), 2.49 (dt, $J=14.5$, $4.1 \mathrm{~Hz}, 1 \mathrm{H}), 2.04(\mathrm{ddd}, J=14.9,10.5,4.8 \mathrm{~Hz}, 1 \mathrm{H}), 1.32(\mathrm{~s}, 3 \mathrm{H}), 0.52(\mathrm{dd}, J=9.4,5.1 \mathrm{~Hz}, 1 \mathrm{H}), 0.42(\mathrm{t}, J=$ $5.7 \mathrm{~Hz}, 1 \mathrm{H}), 0.35(\mathrm{dd}, J=9.7,6.2 \mathrm{~Hz}, 1 \mathrm{H}) .{ }^{13} \mathbf{C}$ NMR $\left(125 \mathrm{MHz} \mathrm{CDCl}_{3}\right) \delta 139.4,133.7,130.0,129.0$, $127.8,126.0,125.6,125.2,123.2,122.9,70.1,54.5,36.0,25.7,20.5,20.1$. IR (neat, $\mathrm{cm}^{-1}$ ): $v=3297,2952$, 2923, 1444, 1376, 1263, 1052, 1017, 963, 791, 776. HRMS (ESI): $m / z 265.1199$ calc. for $\mathrm{C}_{16} \mathrm{H}_{18} \mathrm{NaO}_{2}{ }^{+}$ $[\mathrm{M}+\mathrm{Na}]^{+}$, found 265.1202 .

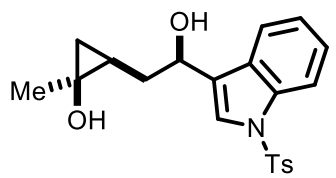

15g: 410 mg; 40\%; white solid; purified by column chromatography (hexane $\left./ \mathrm{Et}_{2} \mathrm{O}=2 / 1\right)$. ${ }^{1} \mathbf{H}$ NMR (500 $\left.\mathrm{MHz}, \mathrm{CDCl}_{3}\right) \delta 8.01(\mathrm{~d}, J=8.3 \mathrm{~Hz}, 1 \mathrm{H}), 7.76(\mathrm{~d}, J=8.4 \mathrm{~Hz}, 2 \mathrm{H}), 7.61-7.51(\mathrm{~m}, 2 \mathrm{H}), 7.36-7.29(\mathrm{~m}, 1 \mathrm{H})$, $7.27-7.15$ (m, 3H), 5.18 (t, J=4.3 Hz, 1H), 2.88 (s, 2H), $2.42-2.27$ (m, 4H), $2.01-1.92(\mathrm{~m}, 1 \mathrm{H}), 1.34$ $(\mathrm{s}, 3 \mathrm{H}), 0.55(\mathrm{dd}, J=9.2,5.1 \mathrm{~Hz}, 1 \mathrm{H}), 0.47-0.33(\mathrm{~m}, 2 \mathrm{H}) .{ }^{13} \mathbf{C}$ NMR $\left(125 \mathrm{MHz}, \mathrm{CDCl}_{3}\right) \delta 145.1,135.8$, 135.4, 130.0, 128.7, 127.0, 126.1, 125.0, 123.3, 123.1, 120.1, 114.0, 67.8, 54.7, 35.3, 25.9, 21.7, 20.4, 20.2. IR (neat, $\left.\mathrm{cm}^{-1}\right): v=3320,2968,2922,1447,1367,1271,1173,1121,748,665,591,572$. HRMS (ESI): $m / z 408.1240$ calc. for $\mathrm{C}_{21} \mathrm{H}_{23} \mathrm{NO}_{4} \mathrm{SNa}^{+}[\mathrm{M}+\mathrm{Na}]^{+}$, found 408.1240 . 


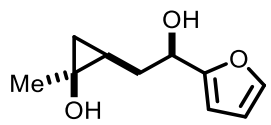

15h: 313 mg; 31\%; colorless oil; purified by column chromatography (hexane/Et $\left.t_{2} \mathrm{O}=2 / 1\right)$. ${ }^{1} \mathbf{H}$ NMR (500 $\left.\mathrm{MHz}, \mathrm{CDCl}_{3}\right) \delta 7.38(\mathrm{dd}, J=1.8,0.9 \mathrm{~Hz}, 1 \mathrm{H}), 6.35(\mathrm{dd}, J=3.2,1.8 \mathrm{~Hz}, 1 \mathrm{H}), 6.28(\mathrm{dt}, J=3.3,0.8 \mathrm{~Hz}, 1 \mathrm{H})$, 4.88 (ddd, $J=5.3,4.4,0.8 \mathrm{~Hz}, 1 \mathrm{H}), 2.79$ (s, 2H), 2.29 (dt, $J=14.3,4.4 \mathrm{~Hz}, 1 \mathrm{H}), 1.98-1.79$ (m, $1 \mathrm{H}), 1.33$ $(\mathrm{d}, J=0.7 \mathrm{~Hz}, 3 \mathrm{H}), 0.73-0.53(\mathrm{~m}, 2 \mathrm{H}), 0.44(\mathrm{ddd}, J=5.4,4.5,0.8 \mathrm{~Hz}, 1 \mathrm{H}) .{ }^{13} \mathbf{C} \mathbf{~ N M R}\left(125 \mathrm{MHz}, \mathrm{CDCl}_{3}\right)$ $\delta 156.8,141.8,110.4,106.0,68.0,54.7,34.4,25.8,20.7,20.1$. IR $\left(\right.$ neat, $\left.\mathrm{cm}^{-1}\right): v=3319,2955,2923,1445$, 1376, 1266, 1149, 1055, 1008, 738. HRMS (ESI): $\mathrm{m} / \mathrm{z} 205.0835$ calc. for $\mathrm{C}_{10} \mathrm{H}_{14} \mathrm{O}_{3} \mathrm{Na}^{+}[\mathrm{M}+\mathrm{Na}]^{+}$, found 205.0836 .

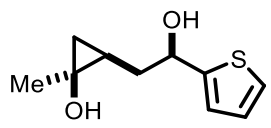

15i: $345 \mathrm{mg}$; 40\%; colorless oil; purified by column chromatography (hexane/Et $\left.{ }_{2} \mathrm{O}=2 / 1\right){ }^{1} \mathbf{H}$ NMR (500 $\left.\mathrm{MHz}, \mathrm{CDCl}_{3}\right) \delta 7.23(\mathrm{dd}, J=4.8,1.4 \mathrm{~Hz}, 1 \mathrm{H}), 7.00-6.98(\mathrm{~m}, 2 \mathrm{H}), 5.17(\mathrm{t}, J=4.4 \mathrm{~Hz}, 1 \mathrm{H}), 3.01(\mathrm{~s}, 2 \mathrm{H})$, $2.29-2.24(\mathrm{~m}, 1 \mathrm{H}), 1.95-1.89(\mathrm{~m}, 1 \mathrm{H}), 1.36(\mathrm{~s}, 3 \mathrm{H}), 0.63-0.60(\mathrm{~m}, 2 \mathrm{H}), 0.46-0.44(\mathrm{~m}, 1 \mathrm{H}) .{ }^{13} \mathbf{C} \mathbf{~ N M R}$ $\left(125 \mathrm{MHz}, \mathrm{CDCl}_{3}\right) \delta 148.5,126.8,124.2,123.3,70.3,54.7,37.8,25.8,20.4,20.1$. IR (neat, $\left.\mathrm{cm}^{-1}\right): v=3307$, 2954, 2916, 1444, 1376, 1266, 1034, 963, 697. HRMS (ESI): $\mathrm{m} / z 181.0682$ calc. for $\mathrm{C}_{10} \mathrm{H}_{13} \mathrm{OS}^{+}[\mathrm{M}+\mathrm{H}-$ $\left.\mathrm{H}_{2} \mathrm{O}\right]^{+}$, found 181.0679 .

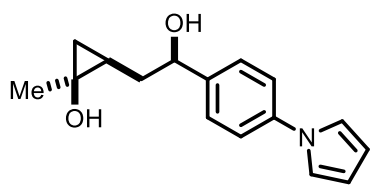

15j: $236 \mathrm{mg}$; 34\%; white solid; purified by column chromatography (hexane/ $\left.\mathrm{Et}_{2} \mathrm{O}=2 / 1\right)$. ${ }^{1} \mathbf{H}$ NMR (500 $\left.\mathrm{MHz}, \mathrm{CDCl}_{3}\right) \delta 7.46-7.35(\mathrm{~m}, 4 \mathrm{H}), 7.09(\mathrm{t}, J=2.2 \mathrm{~Hz}, 2 \mathrm{H}), 6.35(\mathrm{t}, J=2.2 \mathrm{~Hz}, 2 \mathrm{H}), 4.96(\mathrm{t}, J=4.5 \mathrm{~Hz}$, 1H), 3.14 (s, 2H), 2.24 (dt, $J=14.6,4.1 \mathrm{~Hz}, 1 \mathrm{H}), 1.89$ (ddd, $J=14.6,9.8,5.1 \mathrm{~Hz}, 1 \mathrm{H}), 1.35$ (s, 3H), 0.63 $0.53(\mathrm{~m}, 1 \mathrm{H}), 0.47-0.38(\mathrm{~m}, 2 \mathrm{H}) .{ }^{13} \mathbf{C}$ NMR $\left(125 \mathrm{MHz}, \mathrm{CDCl}_{3}\right) \delta 141.5,139.8,126.9,120.2,119.3,110.4$, 72.9, 54.4, 37.3, 25.7, 20.1. IR (neat, $\mathrm{cm}^{-1}$ ): $v=3305,2916,1521,1327,1270,1070,1049,1019,723$. HRMS (ESI): $m / z 258.1489$ calc. for $\mathrm{C}_{16} \mathrm{H}_{20} \mathrm{NO}_{2}{ }^{+}[\mathrm{M}+\mathrm{H}]^{+}$, found 258.1490 .

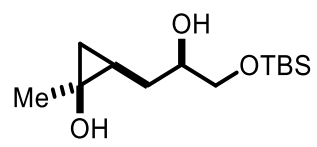

15k: $98 \mathrm{mg}$; 28\%; colorless liquid; purified by column chromatography (hexane/Et $2 \mathrm{O}=2 / 1) .{ }^{1} \mathbf{H}$ NMR (500 $\left.\mathrm{MHz}, \mathrm{CDCl}_{3}\right) \delta 3.79-3.74(\mathrm{~m}, 1 \mathrm{H}), 3.68-3.59(\mathrm{~m}, 2 \mathrm{H}), 2.84(\mathrm{~s}, 1 \mathrm{H}), 1.94(\mathrm{dt}, J=14.7,4.1 \mathrm{~Hz}, 1 \mathrm{H}), 1.60$ 
$-1.55(\mathrm{~m}, 1 \mathrm{H}), 1.36(\mathrm{~d}, J=0.7 \mathrm{~Hz}, 3 \mathrm{H}), 0.89(\mathrm{~s}, 9 \mathrm{H}), 0.59(\mathrm{dd}, J=4.0,2.2 \mathrm{~Hz}, 2 \mathrm{H}), 0.39(\mathrm{dt}, J=4.2,2.5$ $\mathrm{Hz}, 1 \mathrm{H}), 0.08(\mathrm{~d}, J=2.1 \mathrm{~Hz}, 6 \mathrm{H}) .{ }^{13} \mathbf{C}$ NMR $\left(125 \mathrm{MHz}, \mathrm{CDCl}_{3}\right) \delta 71.5,66.1,54.0,31.0,25.9(2 \mathrm{C}), 20.5$, 20.3, 18.3, -5.3, -5.4. IR (neat, $\mathrm{cm}^{-1}$ ): $v=3320,2929,2162,2021,2001,1978,1955,837,549,469$. HRMS (ESI): $m / z 243.1775$ calc. for $\mathrm{C}_{13} \mathrm{H}_{27} \mathrm{O}_{2} \mathrm{Si}^{+}\left[\mathrm{M}+\mathrm{H}-\mathrm{H}_{2} \mathrm{O}\right]^{+}$, found 243.1774 .

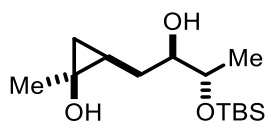

15l: $224 \mathrm{mg} ; 21 \%$; colorless oil; purified by column chromatography (hexane/ $\left.\mathrm{Et}_{2} \mathrm{O}=2 / 1\right) .{ }^{1} \mathbf{H}$ NMR (500 $\left.\mathrm{MHz}, \mathrm{CDCl}_{3}\right) \delta 3.86-3.82(\mathrm{~m}, 1 \mathrm{H}), 3.61-3.58(\mathrm{~m}, 1 \mathrm{H}), 1.86(\mathrm{ddd}, J=14.6,6.4,5.1 \mathrm{~Hz}, 1 \mathrm{H}), 1.55(\mathrm{ddd}$, $J=14.4,9.1,4.8 \mathrm{~Hz}, 1 \mathrm{H}), 1.41(\mathrm{~s}, 3 \mathrm{H}), 1.15$ (d, $J=6.3 \mathrm{~Hz}, 3 \mathrm{H}), 0.89$ (s, 9H), 0.76 (tdd, $J=9.2,6.2,5.1$ $\mathrm{Hz}, 1 \mathrm{H}), 0.62(\mathrm{dd}, J=9.3,5.3 \mathrm{~Hz}, 1 \mathrm{H}), 0.41(\mathrm{t}, J=5.8 \mathrm{~Hz}, 1 \mathrm{H}), 0.08(\mathrm{~s}, 3 \mathrm{H}), 0.08(\mathrm{~s}, 3 \mathrm{H}) ;{ }^{13} \mathbf{C} \mathbf{N M R}(125$ $\left.\mathrm{MHz}, \mathrm{CDCl}_{3}\right) \delta 75.4,71.1,54.8,30.4,26.1,26.0,21.3,19.9,18.2,-4.2,-4.7$. IR (neat, $\left.\mathrm{cm}^{-1}\right): v=3318$, 2955, 2929, 2858, 1463, 1374, 1256, 1086, 967, 834, 775. HRMS (ESI): $m / z ~ 297.1856$ calc. for $\mathrm{C}_{14} \mathrm{H}_{30} \mathrm{O}_{3} \mathrm{NaSi}^{+}[\mathrm{M}+\mathrm{Na}]^{+}$, found 297.1857.

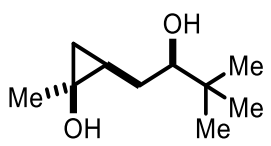

15m: $208 \mathrm{mg}$; 23\%; white solid; purified by column chromatography (hexane/ $\left.\mathrm{Et}_{2} \mathrm{O}=2 / 1\right) .{ }^{1} \mathbf{H}$ NMR (500 $\left.\mathrm{MHz}, \mathrm{CDCl}_{3}\right) \delta 3.36(\mathrm{dd}, J=9.1,2.6 \mathrm{~Hz}, 1 \mathrm{H}), 2.09(\mathrm{~s}, 1 \mathrm{H}), 1.83(\mathrm{~s}, 1 \mathrm{H}), 1.74(\mathrm{ddd}, J=14.6,8.1,2.6 \mathrm{~Hz}$, 1H), 1.62 (dd, $J=9.0,5.7 \mathrm{~Hz}, 1 \mathrm{H}), 1.43$ (d, $J=0.7 \mathrm{~Hz}, 3 \mathrm{H}), 0.93$ (s, 9H), $0.85-0.77$ (m, 1H), 0.66 (dd, $J$ $=9.2,5.2 \mathrm{~Hz}, 1 \mathrm{H}), 0.44$ (t, $J=5.7 \mathrm{~Hz}, 1 \mathrm{H}) .{ }^{13} \mathbf{C} \mathbf{~ N M R}\left(125 \mathrm{MHz}, \mathrm{CDCl}_{3}\right) \delta 80.2,54.5,35.1,30.4,26.0$, 25.9, 22.5, 19.9. IR (neat, $\mathrm{cm}^{-1}$ ): $v=3340,2954,2869,1363,1259,1238,1091,1075,1028,1018,1007$, 991, 957, 871. HRMS (ESI): $m / z 155.1430$ calc. for $\mathrm{C}_{10} \mathrm{H}_{19} \mathrm{O}^{+}\left[\mathrm{M}+\mathrm{H}-\mathrm{H}_{2} \mathrm{O}\right]^{+}$, found 155.1432 .

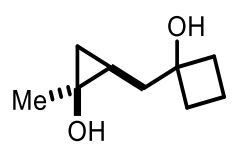

15n: $436 \mathrm{mg}$; 42\%; colorless liquid; purified by column chromatography (hexane/ $\mathrm{Et}_{2} \mathrm{O}=2 / 1$ ). ${ }^{1} \mathbf{H}$ NMR $\left(500 \mathrm{MHz}, \mathrm{CDCl}_{3}\right) \delta 3.60(\mathrm{~s}, 1 \mathrm{H}), 2.88(\mathrm{~s}, 1 \mathrm{H}), 2.26-2.12(\mathrm{~m}, 2 \mathrm{H}), 2.10-1.95(\mathrm{~m}, 3 \mathrm{H}), 1.75-1.66(\mathrm{~m}$, 1H), $1.56-1.47(\mathrm{~m}, 2 \mathrm{H}), 1.36(\mathrm{~d}, J=0.6 \mathrm{~Hz}, 3 \mathrm{H}), 0.75-0.67(\mathrm{~m}, 1 \mathrm{H}), 0.64$ (dd, $J=9.2,5.0 \mathrm{~Hz}, 1 \mathrm{H}), 0.48$ $-0.40(\mathrm{~m}, 1 \mathrm{H}) .{ }^{13} \mathbf{C}$ NMR $\left(125 \mathrm{MHz}, \mathrm{CDCl}_{3}\right) \delta$ 75.1, 54.2, 37.8, 37.6, 35.5, 25.9, 20.4, 20.1, 12.0. IR (neat, $\left.\mathrm{cm}^{-1}\right): v=3346,2933,1371,1272,1244,1165,1111,1020,956$. HRMS (ESI): $\mathrm{m} / z 139.1117$ calc. for $\mathrm{C}_{9} \mathrm{H}_{15} \mathrm{O}^{+}\left[\mathrm{M}+\mathrm{H}-\mathrm{H}_{2} \mathrm{O}\right]^{+}$, found 139.1118 . 


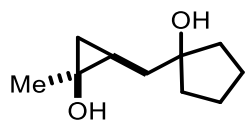

15o: $421 \mathrm{mg}$; 52\%; colorless oil; purified by column chromatography (hexane/ $\left.\mathrm{Et}_{2} \mathrm{O}=2 / 1\right)$. ${ }^{1} \mathbf{H}$ NMR (500 $\left.\mathrm{MHz}, \mathrm{CDCl}_{3}\right) \delta 2.70(\mathrm{~s}, 2 \mathrm{H}), 1.92(\mathrm{dd}, J=14.6,4.3 \mathrm{~Hz}, 1 \mathrm{H}), 1.82-1.76(\mathrm{~m}, 2 \mathrm{H}), 1.73-1.55(\mathrm{~m}, 7 \mathrm{H}), 1.39$ $(\mathrm{s}, 3 \mathrm{H}), 0.76-0.71(\mathrm{~m}, 1 \mathrm{H}), 0.64(\mathrm{dd}, J=9.2,5.1 \mathrm{~Hz}, 1 \mathrm{H}), 0.41(\mathrm{t}, J=5.6 \mathrm{~Hz}, 1 \mathrm{H}) .{ }^{13} \mathbf{C ~ N M R}(125 \mathrm{MHz}$, $\left.\mathrm{CDCl}_{3}\right) \delta 83.0,54.3,41.2,40.0,38.8,26.2,23.8,23.6,21.7,20.6$. IR (neat, $\mathrm{cm}^{-1}$ ): $v=3319,2956,2873$, 1445, 1375, 1272, 1016, 958. HRMS (ESI): $\mathrm{m} / z 153.1274$ calc. for $\mathrm{C}_{10} \mathrm{H}_{17} \mathrm{O}^{+}\left[\mathrm{M}+\mathrm{H}_{-} \mathrm{H}_{2} \mathrm{O}\right]^{+}$, found 153.1273 .

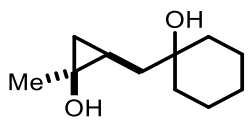

15p: $309 \mathrm{mg}$; 26\%; colorless liquid; purified by column chromatography (hexane/Et $\mathrm{E}_{2} \mathrm{O}=2 / 1$ ). ${ }^{1} \mathbf{H}$ NMR $\left(500 \mathrm{MHz}, \mathrm{CDCl}_{3}\right) \delta 4.24(\mathrm{~s}, 1 \mathrm{H}), 2.60(\mathrm{~s}, 1 \mathrm{H}), 1.99(\mathrm{dd}, J=14.8,4.4 \mathrm{~Hz}, 1 \mathrm{H}), 1.65-1.57(\mathrm{~m}, 1 \mathrm{H}), 1.57$ - $1.38(\mathrm{~m}, 8 \mathrm{H}), 1.38-1.24$ (m, 5H), 0.68 (dddd, $J=10.6,9.2,6.1,4.4 \mathrm{~Hz}, 1 \mathrm{H}), 0.59$ (dd, $J=9.3,5.0 \mathrm{~Hz}$, $1 \mathrm{H}), 0.37(\mathrm{t}, J=5.5 \mathrm{~Hz}, 1 \mathrm{H}) .{ }^{13} \mathbf{C}$ NMR $\left(125 \mathrm{MHz}, \mathrm{CDCl}_{3}\right) \delta$ 72.0, 54.0, 40.3, 39.4, 36.1, 26.0, 25.8, 22.7, 22.3, 20.6, 20.0. IR (neat, $\mathrm{cm}^{-1}$ ): $v=3342,2928,2858,1447,1375,1273,1162,1047,966$. HRMS (ESI): $m / z 207.1356$ calc. for $\mathrm{C}_{11} \mathrm{H}_{20} \mathrm{NaO}_{2}{ }^{+}[\mathrm{M}+\mathrm{Na}]^{+}$, found 207.1357 .

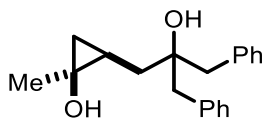

15q: $124 \mathrm{mg}$; 14\%; white solid; purified by column chromatography (hexane/Et $\left.\mathrm{Et}_{2} \mathrm{O}=2 / 1\right){ }^{1} \mathbf{H}$ NMR (500 $\left.\mathrm{MHz}, \mathrm{CDCl}_{3}\right) \delta 7.39-7.10(\mathrm{~m}, 10 \mathrm{H}), 3.03(\mathrm{~d}, J=13.6 \mathrm{~Hz}, 1 \mathrm{H}), 2.87(\mathrm{~d}, J=13.5 \mathrm{~Hz}, 1 \mathrm{H}), 2.74$ (q, $J=13.7$ $\mathrm{Hz}, 2 \mathrm{H}), 1.95$ (dd, $J=14.8,4.3 \mathrm{~Hz}, 1 \mathrm{H}), 1.43$ (dd, $J=14.9,10.5 \mathrm{~Hz}, 1 \mathrm{H}), 1.39$ (d, $J=0.6 \mathrm{~Hz}, 3 \mathrm{H}), 0.77$ (dddd, $J=10.5,9.2,6.1,4.3 \mathrm{~Hz}, 1 \mathrm{H}), 0.63(\mathrm{dd}, J=9.2,5.1 \mathrm{~Hz}, 1 \mathrm{H}), 0.33(\mathrm{t}, J=5.7 \mathrm{~Hz}, 1 \mathrm{H}) .{ }^{13} \mathbf{C} \mathbf{~ N M R}$ $\left(125 \mathrm{MHz}, \mathrm{CDCl}_{3}\right) \delta 137.4,137.1,130.7,128.4,128.1,126.7,126.5,74.5,54.3,46.7,45.4,38.1,26.0,20.6$, 20.5. IR (neat, $\mathrm{cm}^{-1}$ ): $v=3466,3028,2924,1495,1454,1243,1031,753,701$. HRMS (ESI): $\mathrm{m} / z$ 319.1669 calc. for $\mathrm{C}_{20} \mathrm{H}_{24} \mathrm{NaO}_{2}{ }^{+}[\mathrm{M}+\mathrm{Na}]^{+}$, found 319.1669 .

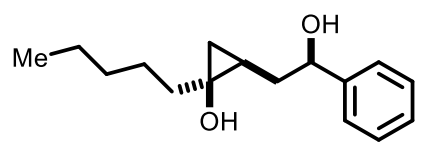

15r: $494 \mathrm{mg}$; 59\%; colorless oil; purified by column chromatography (hexane/ $\left.\mathrm{Et}_{2} \mathrm{O}=2 / 1\right) .{ }^{1} \mathbf{H}$ NMR (500 $\left.\mathrm{MHz}, \mathrm{CDCl}_{3}\right) \delta 7.40-7.34(\mathrm{~m}, 4 \mathrm{H}), 7.28-7.25(\mathrm{~m}, 1 \mathrm{H}), 4.92(\mathrm{t}, J=4.7 \mathrm{~Hz}, 1 \mathrm{H}), 2.80(\mathrm{~s}, 2 \mathrm{H}), 2.23(\mathrm{dt}, J$ $=14.5,4.3 \mathrm{~Hz}, 1 \mathrm{H}), 1.89$ (ddd, $J=14.5,10.0,5.3 \mathrm{~Hz}, 1 \mathrm{H}), 1.56-1.49(\mathrm{~m}, 1 \mathrm{H}), 1.48-1.40(\mathrm{~m}, 2 \mathrm{H}), 1.36$ $-1.23(\mathrm{~m}, 5 \mathrm{H}), 0.89(\mathrm{t}, J=7.0 \mathrm{~Hz}, 3 \mathrm{H}), 0.55(\mathrm{dd}, J=9.2,5.0 \mathrm{~Hz}, 1 \mathrm{H}), 0.49-0.42(\mathrm{~m}, 1 \mathrm{H}), 0.37(\mathrm{t}, J=5.5$ 
$\mathrm{Hz}, 1 \mathrm{H}) .{ }^{13} \mathbf{C}$ NMR $\left(125 \mathrm{MHz}, \mathrm{CDCl}_{3}\right) \delta 144.2,128.4,127.3,125.9,73.6,58.2,39.5,37.3,32.1,25.4,22.8$, 19.4, 19.1, 14.2. IR (neat, $\mathrm{cm}^{-1}$ ): $v=3318,2954,2929,1452,1249,1044,700$. HRMS (ESI): $\mathrm{m} / z 271.1669$ calc. for $\mathrm{C}_{16} \mathrm{H}_{24} \mathrm{O}_{2} \mathrm{Na}^{+}[\mathrm{M}+\mathrm{Na}]^{+}$, found 271.1670.

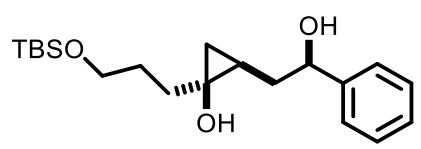

15s: $614 \mathrm{mg}$; 35\%; colorless oil; purified by column chromatography (hexane/ $\left.\mathrm{Et}_{2} \mathrm{O}=2 / 1\right)$. ${ }^{1} \mathbf{H}$ NMR (500 $\left.\mathrm{MHz}, \mathrm{CDCl}_{3}\right) \delta 7.43-7.31(\mathrm{~m}, 4 \mathrm{H}), 7.26-7.21(\mathrm{~m}, 1 \mathrm{H}), 4.92(\mathrm{t}, J=4.6 \mathrm{~Hz}, 1 \mathrm{H}), 3.79-3.63(\mathrm{~m}, 2 \mathrm{H})$, $2.22(\mathrm{dt}, J=14.6,4.6 \mathrm{~Hz}, 1 \mathrm{H}), 2.02-1.88(\mathrm{~m}, 1 \mathrm{H}), 1.84-1.76(\mathrm{~m}, 1 \mathrm{H}), 1.76-1.69(\mathrm{~m}, 2 \mathrm{H}), 1.39-1.31$ (m, 1H), $0.91(\mathrm{~s}, 9 \mathrm{H}), 0.55(\mathrm{dd}, J=9.2,4.9 \mathrm{~Hz}, 1 \mathrm{H}), 0.47-0.40(\mathrm{~m}, 1 \mathrm{H}), 0.41-0.32(\mathrm{~m}, 1 \mathrm{H}), 0.09(\mathrm{~s}, 6 \mathrm{H})$. ${ }^{13}$ C NMR (125 MHz, $\left.\mathrm{CDCl}_{3}\right) \delta 144.8,128.2,127.0,125.9,73.4,64.0,57.8,37.5,37.4,29.7,26.1,19.7$, 19.0, 18.5, -5.2, -5.3. IR (neat, $\mathrm{cm}^{-1}$ ): $v=3325,2952,2928,2856,1471,1451,1255,1096,1043,1004$, 835, 776, 80. HRMS (ESI): $m / z 351.2350$ calc. for $\mathrm{C}_{20} \mathrm{H}_{35} \mathrm{O}_{3} \mathrm{Si}^{+}[\mathrm{M}+\mathrm{H}]^{+}$, found 351.2351.

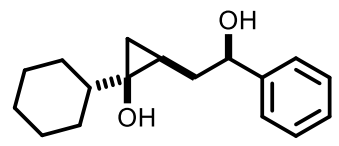

15t: $505 \mathrm{mg} ; 29 \%$; colorless solid; purified by column chromatography (hexane/Et $\left.\mathrm{E}_{2} \mathrm{O}=2 / 1\right) .{ }^{1} \mathbf{H}$ NMR (500 $\left.\mathrm{MHz}, \mathrm{CDCl}_{3}\right) \delta 7.43-7.31(\mathrm{~m}, 4 \mathrm{H}), 7.29-7.23(\mathrm{~m}, 1 \mathrm{H}), 4.91(\mathrm{t}, J=4.9 \mathrm{~Hz}, 1 \mathrm{H}), 2.33-2.19(\mathrm{~m}, 3 \mathrm{H})$, $1.96-1.88(\mathrm{~m}, 1 \mathrm{H}), 1.80-1.72(\mathrm{~m}, 3 \mathrm{H}), 1.68-1.59(\mathrm{~m}, 2 \mathrm{H}), 1.23-1.09(\mathrm{~m}, 5 \mathrm{H}), 0.86-0.78(\mathrm{~m}, 1 \mathrm{H})$, $0.60-0.46(\mathrm{~m}, 2 \mathrm{H}), 0.32(\mathrm{~d}, J=3.0 \mathrm{~Hz}, 1 \mathrm{H}) .{ }^{13} \mathbf{C}$ NMR $\left(125 \mathrm{MHz}, \mathrm{CDCl}_{3}\right) \delta 144.3,128.4,127.3,125.8$, 73.6, 62.0, 46.2, 37.3, 28.8, 28.7, 26.6, 26.5, 18.7, 18.3. IR (neat, $\left.\mathrm{cm}^{-1}\right): v=3332,2925,2852,1450,1260$, 1046, 755, 701. HRMS (ESI): $m / z 283.1669$ calc. for $\mathrm{C}_{17} \mathrm{H}_{24} \mathrm{NaO}_{2}{ }^{+}[\mathrm{M}+\mathrm{Na}]^{+}$, found 283.1668.

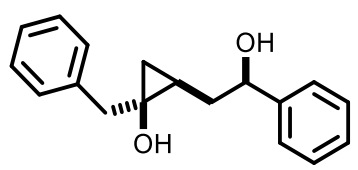

15u: $473 \mathrm{mg}$; 52\%; colorless oil; purified by column chromatography (hexane/Et $2 \mathrm{O}=2 / 1) .{ }^{1} \mathbf{H}$ NMR (500 $\left.\mathrm{MHz}, \mathrm{CDCl}_{3}\right) \delta 7.38-7.22(\mathrm{~m}, 10 \mathrm{H}), 4.87(\mathrm{t}, J=4.8 \mathrm{~Hz}, 1 \mathrm{H}), 2.87(\mathrm{~d}, J=14.1 \mathrm{~Hz}, 1 \mathrm{H}), 2.76(\mathrm{~d}, J=14.0$ $\mathrm{Hz}, 1 \mathrm{H}), 2.35$ (s, 2H), 2.23 (dt, $J=14.6,4.5 \mathrm{~Hz}, 1 \mathrm{H}), 1.90(\mathrm{ddd}, J=14.8,10.0,5.1 \mathrm{~Hz}, 1 \mathrm{H}), 0.75(\mathrm{dd}, J=$ 9.4, $5.2 \mathrm{~Hz}, 1 \mathrm{H}), 0.68-0.61(\mathrm{~m}, 1 \mathrm{H}), 0.46(\mathrm{t}, J=5.3 \mathrm{~Hz}, 1 \mathrm{H}) .{ }^{13} \mathbf{C} \mathbf{N M R}\left(125 \mathrm{MHz}, \mathrm{CDCl}_{3}\right) \delta 144.1,138.7$, 129.7, 128.5, 128.4, 127.4, 126.6, 125.8, 73.5, 58.5, 45.1, 37.2, 19.0, 18.9. IR (neat, $\mathrm{cm}^{-1}$ ): $v=3346,2923$, 1495, 1453, 1046, 1030, 755, 701. HRMS (ESI): $m / z 291.1356$ calc. for $\mathrm{C}_{18} \mathrm{H}_{20} \mathrm{O}_{2} \mathrm{Na}^{+}[\mathrm{M}+\mathrm{Na}]^{+}$, found 291.1355 . 


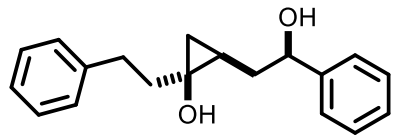

15v: $577 \mathrm{mg}$; 66\%; colorless liquid; purified by column chromatography (hexane/Et ${ }_{2} \mathrm{O}=2 / 1$ ). ${ }^{1} \mathbf{H}$ NMR $\left(500 \mathrm{MHz}, \mathrm{CDCl}_{3}\right) \delta 7.41-7.32(\mathrm{~m}, 4 \mathrm{H}), 7.32-7.26(\mathrm{~m}, 3 \mathrm{H}), 7.19(\mathrm{~d}, J=7.2 \mathrm{~Hz}, 3 \mathrm{H}), 4.95(\mathrm{t}, J=4.7 \mathrm{~Hz}$, 1H), $2.90-2.70(\mathrm{~m}, 3 \mathrm{H}), 2.62(\mathrm{~d}, J=49.9 \mathrm{~Hz}, 1 \mathrm{H}), 2.27-2.15(\mathrm{~m}, 1 \mathrm{H}), 1.94-1.80(\mathrm{~m}, 2 \mathrm{H}), 1.65-1.61$ $(\mathrm{m}, 1 \mathrm{H}), 0.58(\mathrm{dd}, J=9.2,5.0 \mathrm{~Hz}, 1 \mathrm{H}), 0.53-0.43(\mathrm{~m}, 1 \mathrm{H}), 0.42(\mathrm{ddd}, J=6.2,4.9,1.4 \mathrm{~Hz}, 1 \mathrm{H}) .{ }^{13} \mathbf{C} \mathbf{~ N M R}$ $\left(125 \mathrm{MHz}, \mathrm{CDCl}_{3}\right) \delta 143.9,142.6,128.4,128.4,128.3,127.3,125.7,125.7,73.5,57.8,41.8,37.1,32.2$, 19.6, 19.1. IR (neat, $\mathrm{cm}^{-1}$ ): $v=3338,3026,2917,1712,1495,1248,1044,749,698$. HRMS (ESI): $\mathrm{m} / z$ 305.1512 calc. for $\mathrm{C}_{19} \mathrm{H}_{22} \mathrm{NaO}_{2}^{+}[\mathrm{M}+\mathrm{Na}]^{+}$, found 305.1511 .

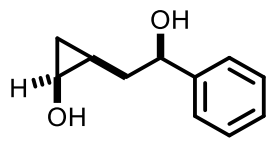

15w: $98 \mathrm{mg}$; 20\%; colorless liquid; purified by column chromatography (hexane/Et $\left.{ }_{2} \mathrm{O}=2 / 1\right)$. ${ }^{1} \mathbf{H}$ NMR (500 $\left.\mathrm{MHz} \mathrm{CDCl}_{3}\right) \delta 7.40(\mathrm{~d}, J=7.5 \mathrm{~Hz}, 2 \mathrm{H}), 7.36(\mathrm{t}, J=7.6 \mathrm{~Hz}, 2 \mathrm{H}), 7.30-7.25(\mathrm{~m}, 1 \mathrm{H}), 4.95(\mathrm{t}, J=4.8 \mathrm{~Hz}, 1 \mathrm{H})$, $3.39(\mathrm{td}, J=6.5,3.2 \mathrm{~Hz}, 1 \mathrm{H}), 2.96(\mathrm{~s}, 2 \mathrm{H}), 2.23(\mathrm{dt}, J=14.4,4.4 \mathrm{~Hz}, 1 \mathrm{H}), 1.87(\mathrm{ddd}, J=14.9,10.2,5.1 \mathrm{~Hz}, 1 \mathrm{H})$, $0.65(\mathrm{dt}, J=9.4,5.9 \mathrm{~Hz}, 1 \mathrm{H}), 0.55-0.45(\mathrm{~m}, 1 \mathrm{H}), 0.27(\mathrm{td}, J=6.1,3.2 \mathrm{~Hz}, 1 \mathrm{H}) .{ }^{13} \mathbf{C} \mathbf{~ N M R}\left(125 \mathrm{MHz}, \mathrm{CDCl}_{3}\right)$ $\delta 143.9,128.3,127.4,125.7,73.7,49.1,36.0,13.5,13.2$. IR (neat, $\mathrm{cm}^{-1}$ ): $v=3317,3027,2920,1452,1324$, 1215, 1040, 755, 701. HRMS (ESI): $m / z 177.0910$ calc. for $\mathrm{C}_{11} \mathrm{H}_{13} \mathrm{O}_{2}{ }^{+}[\mathrm{M}-\mathrm{H}]^{+}$, found 177.0890.

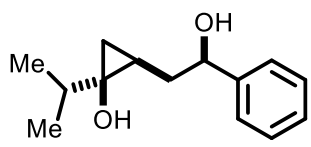

15x: $671 \mathrm{mg}$; 45\%; colorless solid; purified by column chromatography (hexane/Et $\mathrm{Et}_{2} \mathrm{O}=2 / 1$ ). ${ }^{1} \mathbf{H}$ NMR $\left(500 \mathrm{MHz}, \mathrm{CDCl}_{3}\right) \delta 7.41(\mathrm{~d}, J=7.2 \mathrm{~Hz}, 2 \mathrm{H}), 7.36(\mathrm{dd}, J=8.4,6.7 \mathrm{~Hz}, 2 \mathrm{H}), 7.30-7.23(\mathrm{~m}, 1 \mathrm{H}), 4.93(\mathrm{t}$, $J=4.8 \mathrm{~Hz}, 1 \mathrm{H}), 2.25(\mathrm{dt}, J=14.6,4.2 \mathrm{~Hz}, 1 \mathrm{H}), 2.01-1.86(\mathrm{~m}, 1 \mathrm{H}), 1.17(\mathrm{p}, J=6.8 \mathrm{~Hz}, 1 \mathrm{H}), 0.98(\mathrm{~d}, J=$ $6.8 \mathrm{~Hz}, 3 \mathrm{H}), 0.95(\mathrm{~d}, J=6.8 \mathrm{~Hz}, 3 \mathrm{H}), 0.65-0.48(\mathrm{~m}, 2 \mathrm{H}), 0.35(\mathrm{~s}, 1 \mathrm{H}) .{ }^{13} \mathbf{C} \mathbf{N M R}\left(125 \mathrm{MHz}, \mathrm{CDCl}_{3}\right) \delta$ 144.2, 128.4, 127.3, 125.8, 73.5, 62.4, 37.3, 36.2, 19.2, 18.6, 18.4, 18.3. IR (neat, $\mathrm{cm}^{-1}$ ): $v=3331,2961$, 2872, 1451, 1269, 1045, 993, 702. HRMS (ESI): $m / z 203.1430$ calc. for $\mathrm{C}_{14} \mathrm{H}_{19} \mathrm{O}^{+}\left[\mathrm{M}+\mathrm{H}-\mathrm{H}_{2} \mathrm{O}\right]^{+}$, found 203.1431. (Note: $\mathbf{1 5 x}$ and $\mathbf{1 5 y}$ were produced from the same reaction.)

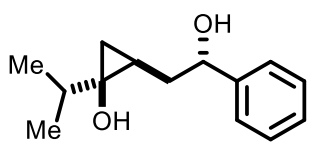


15y: $290 \mathrm{mg}$; 20\%; colorless solid; purified by column chromatography (hexane/Et ${ }_{2} \mathrm{O}=2 / 1$ ). ${ }^{1} \mathbf{H}$ NMR $\left(500 \mathrm{MHz}, \mathrm{CDCl}_{3}\right) \delta 7.38-7.31(\mathrm{~m}, 4 \mathrm{H}), 7.30-7.26(\mathrm{~m}, 1 \mathrm{H}), 4.78(\mathrm{dd}, J=10.5,2.3 \mathrm{~Hz}, 1 \mathrm{H}), 2.17$ (ddd, $J=14.9,4.2,2.3 \mathrm{~Hz}, 1 \mathrm{H}), 1.69$ (dt, $J=14.9,10.5 \mathrm{~Hz}, 1 \mathrm{H}), 1.25$ (s, 1H), 1.19 (h, $J=7.0 \mathrm{~Hz}, 1 \mathrm{H}), 1.06$ (d, $J=6.8 \mathrm{~Hz}, 3 \mathrm{H}), 1.03(\mathrm{~d}, J=6.9 \mathrm{~Hz}, 3 \mathrm{H}), 0.84$ (tdd, $J=10.1,6.0,4.1 \mathrm{~Hz}, 1 \mathrm{H}), 0.63$ (dd, $J=9.3,5.3 \mathrm{~Hz}$, $1 \mathrm{H}), 0.40(\mathrm{t}, J=5.7 \mathrm{~Hz}, 1 \mathrm{H}) .{ }^{13} \mathbf{C}$ NMR $\left(125 \mathrm{MHz}, \mathrm{CDCl}_{3}\right) \delta 145.2,128.7,127.7,125.7,75.5,62.0,39.0$, 36.8, 22.9, 19.4, 18.3, 18.2. IR (neat, $\left.\mathrm{cm}^{-1}\right): v=3338,2962,2930,1970,1451,1270,1061,1003,754,700$. HRMS (ESI): $m / z 203.1430$ calc. for $\mathrm{C}_{14} \mathrm{H}_{19} \mathrm{O}^{+}\left[\mathrm{M}+\mathrm{H}-\mathrm{H}_{2} \mathrm{O}\right]^{+}$, found 203.1432 .

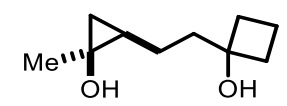

15za: $314 \mathrm{mg}$; 58\%; white solid; purified by column chromatography (hexane/ $\left.\mathrm{Et}_{2} \mathrm{O}=2 / 1\right) .{ }^{1} \mathbf{H}$ NMR (500 $\left.\mathrm{MHz}, \mathrm{CDCl}_{3}\right) \delta 2.08-1.95(\mathrm{~m}, 5 \mathrm{H}), 1.74-1.65(\mathrm{~m}, 4 \mathrm{H}), 1.52(\mathrm{dt}, J=11.3,8.8 \mathrm{~Hz}, 1 \mathrm{H}), 1.43(\mathrm{~m}, 4 \mathrm{H})$, $1.28-1.21(\mathrm{~m}, 1 \mathrm{H}), 1.05-0.95(\mathrm{~m}, 1 \mathrm{H}), 0.85(\mathrm{dd}, J=10.0,5.2 \mathrm{~Hz}, 1 \mathrm{H}), 0.09(\mathrm{dd}, J=6.4,5.2 \mathrm{~Hz}, 1 \mathrm{H})$. ${ }^{13}$ C NMR $\left(125 \mathrm{MHz}, \mathrm{CDCl}_{3}\right) \delta 75.2,55.7,39.3,36.1,36.0,25.7,24.0,20.5,20.3,12.1$. IR (neat, $\left.\mathrm{cm}^{-1}\right): v$ $=3316,2979,2931,1443,1376,1258,1224,1167,1075,1022,955,637$. HRMS (ESI): $m / z 153.1274$ calc. for $\mathrm{C}_{10} \mathrm{H}_{17} \mathrm{O}^{+}\left[\mathrm{M}+\mathrm{H}-\mathrm{H}_{2} \mathrm{O}\right]^{+}$, found 153.1274 .

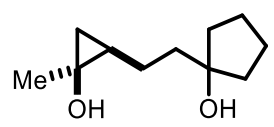

15zb: $327 \mathrm{mg}$; 53\%; colorless oil; purified by column chromatography (hexane/Et $\left.t_{2} \mathrm{O}=2 / 1\right) .{ }^{1} \mathbf{H}$ NMR (500 $\left.\mathrm{MHz}, \mathrm{CDCl}_{3}\right) \delta 1.90-1.75(\mathrm{~m}, 2 \mathrm{H}), 1.73-1.65(\mathrm{~m}, 2 \mathrm{H}), 1.65-1.55(\mathrm{~m}, 5 \mathrm{H}), 1.58-1.51(\mathrm{~m}, 1 \mathrm{H}), 1.49-$ $1.41(\mathrm{~m}, 4 \mathrm{H}), 1.34-1.21(\mathrm{~m}, 1 \mathrm{H}), 1.07-0.95(\mathrm{~m}, 1 \mathrm{H}), 0.84$ (dd, $J=10.0,5.2 \mathrm{~Hz}, 1 \mathrm{H}), 0.08$ (dd, $J=6.4$, $5.2 \mathrm{~Hz}, 1 \mathrm{H}) .{ }^{13} \mathbf{C}$ NMR $\left(125 \mathrm{MHz}, \mathrm{CDCl}_{3}\right) \delta 82.6,55.8,41.5,40.0,39.8,26.0,25.4,23.9,23.9,20.6,20.4$. IR (neat, $\mathrm{cm}^{-1}$ ): $v=3346,2955,2869,1440,1375,1230,1213$, 979. HRMS (ESI): $\mathrm{m} / z$ 207.1356 calc. for $\mathrm{C}_{11} \mathrm{H}_{20} \mathrm{O}_{2} \mathrm{Na}^{+}[\mathrm{M}+\mathrm{Na}]^{+}$, found 207.1357.

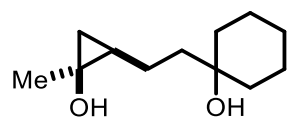

15zc: $187 \mathrm{mg}$; 30\%; white solid; purified by column chromatography (hexane/ $\left.\mathrm{Et}_{2} \mathrm{O}=2 / 1\right) .{ }^{1} \mathbf{H}$ NMR (500 $\left.\mathrm{MHz}, \mathrm{CDCl}_{3}\right) \delta 1.61-1.57(\mathrm{~m}, 1 \mathrm{H}), 1.50-1.16(\mathrm{~m}, 16 \mathrm{H}), 1.00-0.91(\mathrm{~m}, 1 \mathrm{H}), 0.83(\mathrm{dd}, J=10.1,5.2 \mathrm{~Hz}$, 1H), $0.06(\mathrm{dd}, J=6.4,5.1 \mathrm{~Hz}, 1 \mathrm{H}) .{ }^{13} \mathbf{C} \mathbf{N M R}\left(125 \mathrm{MHz}, \mathrm{CDCl}_{3}\right) \delta 71.3,55.6,42.2,37.6,37.3,25.9,25.8$, 23.4, 22.3, 20.5, 20.2. IR (neat, $\mathrm{cm}^{-1}$ ): $v=3351,2928,2859,1447,1227,1164,967$. HRMS (ESI): $\mathrm{m} / z$ 221.1512 calc. for $\mathrm{C}_{12} \mathrm{H}_{22} \mathrm{NaO}_{2}^{+}[\mathrm{M}+\mathrm{Na}]^{+}$, found 221.1527 . 


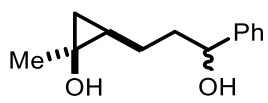

15zd: $612 \mathrm{mg}$ as a 1:1 mixture of diastereomers; $45 \%$; colorless liquid; purified by column chromatography (hexane/Et $\left.\mathrm{E}_{2} \mathrm{O}=2 / 1\right) .{ }^{1} \mathbf{H}$ NMR $\left(500 \mathrm{MHz}, \mathrm{CDCl}_{3}\right) \delta 7.38-7.26(\mathrm{~m}, 5 \mathrm{H}), 4.70$ (ddd, $J=12.1,7.7,5.7 \mathrm{~Hz}$, 1H), $2.01-1.73(\mathrm{~m}, 4 \mathrm{H}), 1.38(\mathrm{~d}, J=11.6 \mathrm{~Hz}, 3 \mathrm{H}), 1.34-1.13(\mathrm{~m}, 2 \mathrm{H}), 0.99(\mathrm{dt}, J=10.0,6.7 \mathrm{~Hz}, 1 \mathrm{H})$, $0.84(\mathrm{ddd}, J=10.2,5.2,2.6 \mathrm{~Hz}, 1 \mathrm{H}), 0.07$ (ddd, $J=8.2,6.5,5.1 \mathrm{~Hz}, 1 \mathrm{H}) .{ }^{13} \mathbf{C} \mathbf{N M R}\left(125 \mathrm{MHz}, \mathrm{CDCl}_{3}\right) \delta$ $144.8,144.7,128.5,127.6,127.6,125.9,125.9,74.4,74.3,55.7,55.6,38.9,38.9,26.2,26.1,25.4,25.3$, 20.5, 20.3, 20.2. IR (neat, $\mathrm{cm}^{-1}$ ): $v=3342,2930,2861,1453,1378,1220,1064,1027,763,701$. HRMS (ESI): $m / z 229.1199$ calc. for $\mathrm{C}_{13} \mathrm{H}_{18} \mathrm{NaO}_{2}^{+}[\mathrm{M}+\mathrm{Na}]^{+}$, found 229.1199 .

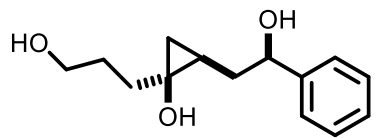

17: To a solution of $15 \mathrm{~s}$ (106 mg, $0.303 \mathrm{mmol}, 1.0$ equiv.) in THF at $0{ }^{\circ} \mathrm{C}$ was added $1.0 \mathrm{M} \mathrm{TBAF}(0.37 \mathrm{ml}$, 1.2 equiv.). The reaction was slowly warmed to room temperature. After $6 \mathrm{~h}$, the reaction was quenched with water, extracted with EtOAc, washed with brine, dried with $\mathrm{Na}_{2} \mathrm{SO}_{4}$, then concentrate under reduced pressure. The residue was purified by flash column (hexane/EtOAc $=1 / 4$ to pure EtOAc) to give triol 17 (65.0 mg, 91\%) as colorless solid. ${ }^{1} \mathbf{H}$ NMR (500 MHz, $\left.\mathrm{CDCl}_{3}\right) \delta 7.40-7.26(\mathrm{~m}, 5 \mathrm{H}), 4.97$ (t, $J=4.5 \mathrm{~Hz}$, 1H), $3.77-3.65$ (m, 2H), $3.03(\mathrm{~s}, 3 \mathrm{H}), 2.24(\mathrm{dt}, J=14.6,4.2 \mathrm{~Hz}, 1 \mathrm{H}), 1.92(\mathrm{dt}, J=9.5,4.8 \mathrm{~Hz}, 1 \mathrm{H}), 1.80$ $-1.73(\mathrm{~m}, 3 \mathrm{H}), 1.45-1.36(\mathrm{~m}, 1 \mathrm{H}), 0.58(\mathrm{dd}, J=9.2,4.9 \mathrm{~Hz}, 1 \mathrm{H}), 0.52-0.44(\mathrm{~m}, 1 \mathrm{H}), 0.42(\mathrm{ddd}, J=6.1$, 4.9, $1.3 \mathrm{~Hz}, 1 \mathrm{H}) .{ }^{13} \mathbf{C} \mathbf{N M R}\left(125 \mathrm{MHz}, \mathrm{CDCl}_{3}\right) \delta 144.1,128.3,127.2,125.7,73.4,63.2,57.8,37.3,37.0$, 29.4, 19.7, 19.0. IR (neat, $\mathrm{cm}^{-1}$ ): $v=3316,2939,1450,1305,1256,1042,1003,755,701$. HRMS (ESI): $m / z 259.1305$ calc. for $\mathrm{C}_{14} \mathrm{H}_{20} \mathrm{NaO}_{3}{ }^{+}[\mathrm{M}+\mathrm{Na}]^{+}$, found 259.1306 .

\section{A representative procedure for the synthesis of bicyclic lactone.}
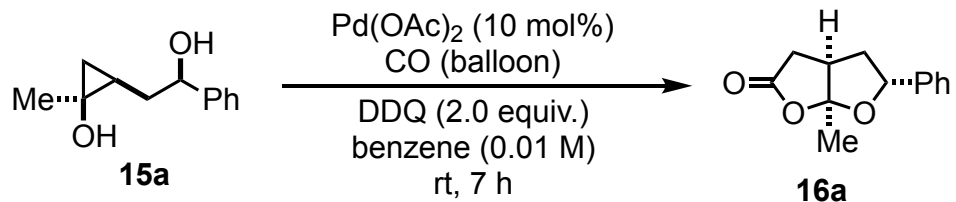

To a stirred solution of $15 \mathrm{a}$ (38.4 mg, $0.2 \mathrm{mmol}, 1.0$ equiv.) and DDQ (90.8 mg, $0.4 \mathrm{mmol}, 2.0$ equiv.) in benzene $(20 \mathrm{ml})$ under carbon monoxide (the reactor was evacuated and backfilled three times using a carbon monoxide balloon) was added $\mathrm{Pd}(\mathrm{OAc})_{2}(4.5 \mathrm{mg}, 0.02 \mathrm{mmol}, 0.1$ equiv.) in one portion. The resulting solution was stirred at room temperature for $7 \mathrm{~h}$. The reaction mixture was quenched with water. The aqueous layer was extracted with ethyl acetate three times. The combined organic layers were washed 
with brine, dried over $\mathrm{Na}_{2} \mathrm{SO}_{4}$, and concentrated under reduced pressure. The resulting residue was purified by flash chromatography (hexane/ethyl acetate $=2 / 1)$ to obtain $\mathbf{1 6 a}(32.3 \mathrm{mg}, 74 \%)$ as colorless solid. ${ }^{1} \mathbf{H}$ NMR $\left(500 \mathrm{MHz}, \mathrm{CDCl}_{3}\right) \delta 7.39-7.29(\mathrm{~m}, 5 \mathrm{H}), 5.16(\mathrm{dd}, J=10.4,5.6 \mathrm{~Hz}, 1 \mathrm{H}), 3.06-2.98(\mathrm{~m}, 1 \mathrm{H}), 2.99$ $(\mathrm{t}, J=2.4 \mathrm{~Hz}, 1 \mathrm{H}), 2.66-2.57(\mathrm{~m}, 1 \mathrm{H}), 2.25-2.10(\mathrm{~m}, 2 \mathrm{H}), 1.78(\mathrm{~s}, 3 \mathrm{H}) .{ }^{13} \mathbf{C} \mathbf{N M R}\left(125 \mathrm{MHz}, \mathrm{CDCl}_{3}\right) \delta$ 174.8, 139.2, 128.6, 128.2, 126.0, 117.2, 80.3, 43.4, 41.9, 36.6, 24.4. IR (neat, $\mathrm{cm}^{-1}$ ): $v=2987,1771,1256$, 1130, 1091, 1007, 919, 760, 701. HRMS (ESI): $\mathrm{m} / z 219.1016$ calc. for $\mathrm{C}_{13} \mathrm{H}_{15} \mathrm{O}_{3}{ }^{+}[\mathrm{M}+\mathrm{H}]^{+}$, found 219.1016

\section{Scale-up synthesis}

To a stirred solution of $\mathbf{1 5 a}$ (560 mg, $2.92 \mathrm{mmol}, 1.0$ equiv.) and DDQ (1.33 g, $5.83 \mathrm{mmol}, 2.0$ equiv.) in benzene $(290 \mathrm{ml})$ under carbon monoxide (the reactor was evacuated and backfilled three times using a carbon monoxide balloon) was added $\mathrm{Pd}(\mathrm{OAc})_{2}(65.6 \mathrm{mg}, 0.292 \mathrm{mmol}, 0.1$ equiv.) in one portion. The resulting solution was stirred at room temperature for $7 \mathrm{~h}$. The reaction mixture was quenched with water. The aqueous layer was extracted with ethyl acetate three times. The combined organic layers were washed with brine, dried over $\mathrm{Na}_{2} \mathrm{SO}_{4}$, and concentrated under reduced pressure. The resulting residue was purified by flash chromatography (hexane/ethyl acetate $=2 / 1$ ) to obtain $\mathbf{1 6 a}(414 \mathrm{mg}, 65 \%)$ as colorless solid.

\section{Asymmetric synthesis}

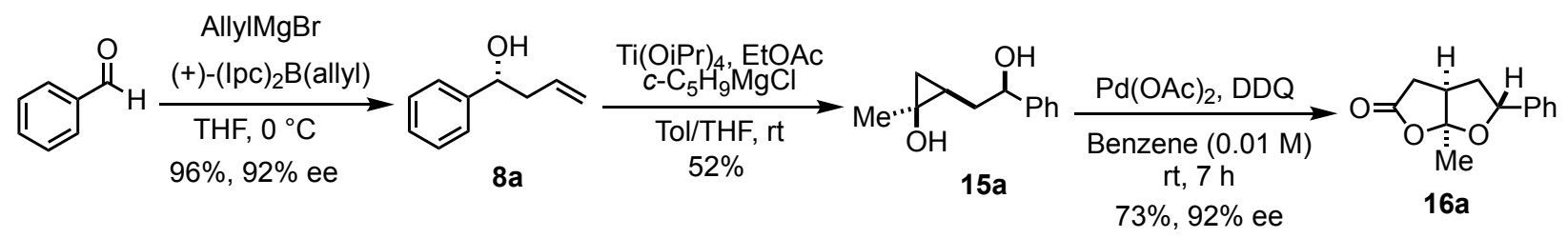

Enantioenriched homoallylic alcohol was prepared according to a reported literature procedure. ${ }^{2}$ Following the above cyclopropanol synthesis and carbonylative lactonization procedures, compound 16a was produced in $73 \%$ yield and $92 \%$ ee. 
8a: SFC conditions: CEL-1, [97:3 to 50:50 CO2:(IPA/MeOH = 1:1)], $8 \mathrm{~min}, 1.5 \mathrm{~mL} / \mathrm{min}$

\begin{tabular}{|c|c|c|c|}
\hline \multicolumn{4}{|c|}{ Racemic 8a Peak Results } \\
\hline & $\mathrm{RT} / \mathrm{min}$ & Area/ $\left(\mu \mathrm{V}^{*} \mathrm{sec}\right)$ & \% Area \\
\hline 1 & 0.853 & 16718 & 50.46 \\
\hline 2 & 0.986 & 16413 & 49.54 \\
\hline
\end{tabular}

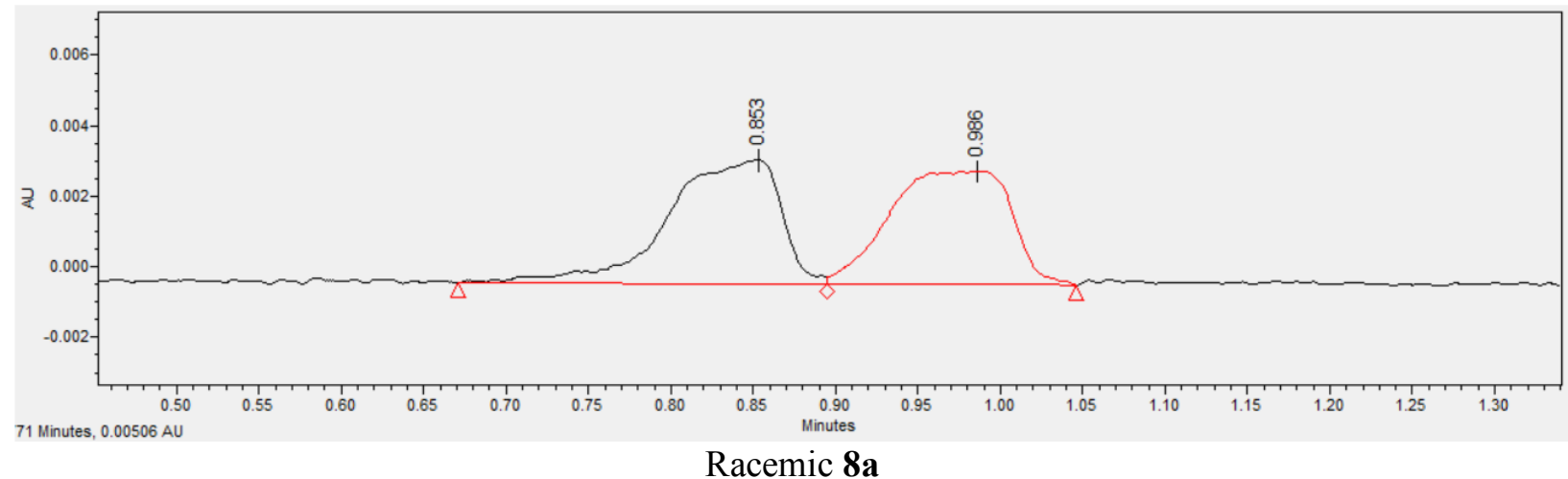

\begin{tabular}{|c|c|c|c|}
\hline \multicolumn{4}{|c|}{ Enantioenriched 8a Peak Results } \\
\hline & RT/min & Area/ $\left(\mu \mathrm{V}^{*} \mathrm{sec}\right)$ & \% Area \\
\hline 1 & 0.853 & 76259 & 96.06 \\
\hline 2 & 0.994 & 3128 & 3.94 \\
\hline
\end{tabular}

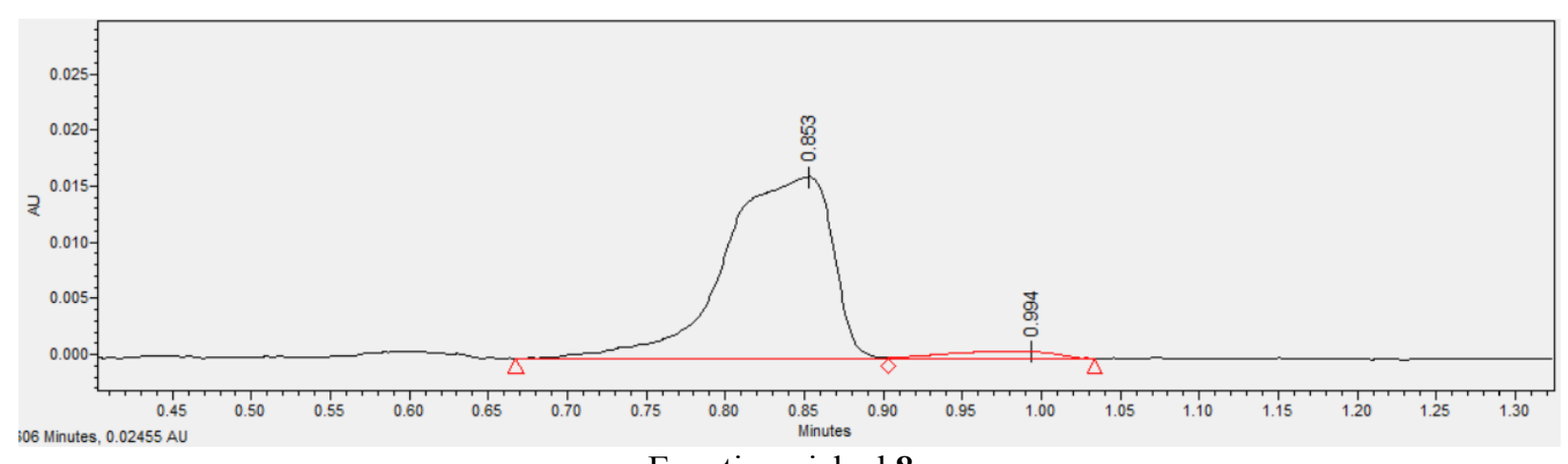

Enantioenriched 8a 
16a: SFC Conditions: CEL-1, [97:3 to 50:50 CO2:(IPA/MeOH = 1:1)], $8 \mathrm{~min}, 1.5 \mathrm{~mL} / \mathrm{min}$

\begin{tabular}{|c|c|c|c|}
\hline \multicolumn{4}{|c|}{ Racemic 16a Peak Results } \\
\hline & RT/min & Area/ $\left(\mu \mathrm{V}^{*} \mathrm{sec}\right)$ & \% Area \\
\hline 1 & 2.091 & 43081 & 51.22 \\
\hline 2 & 2.230 & 41028 & 48.78 \\
\hline
\end{tabular}

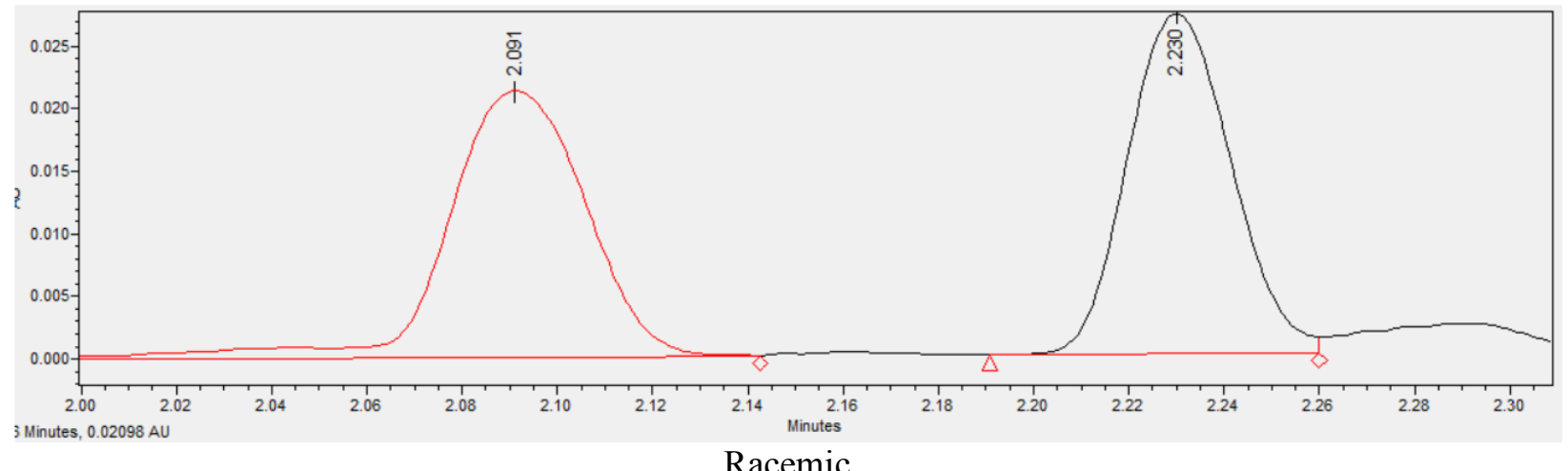

Racemic

\begin{tabular}{|c|c|c|c|}
\hline \multicolumn{4}{|c|}{ Enantioenriched 16a Peak Results } \\
\hline & RT/min & Area/ $\left(\mu \mathrm{V}^{*}\right.$ sec $)$ & \% Area \\
\hline 1 & 2.089 & 29034 & 96.04 \\
\hline 2 & 2.225 & 1196 & 3.96 \\
\hline
\end{tabular}

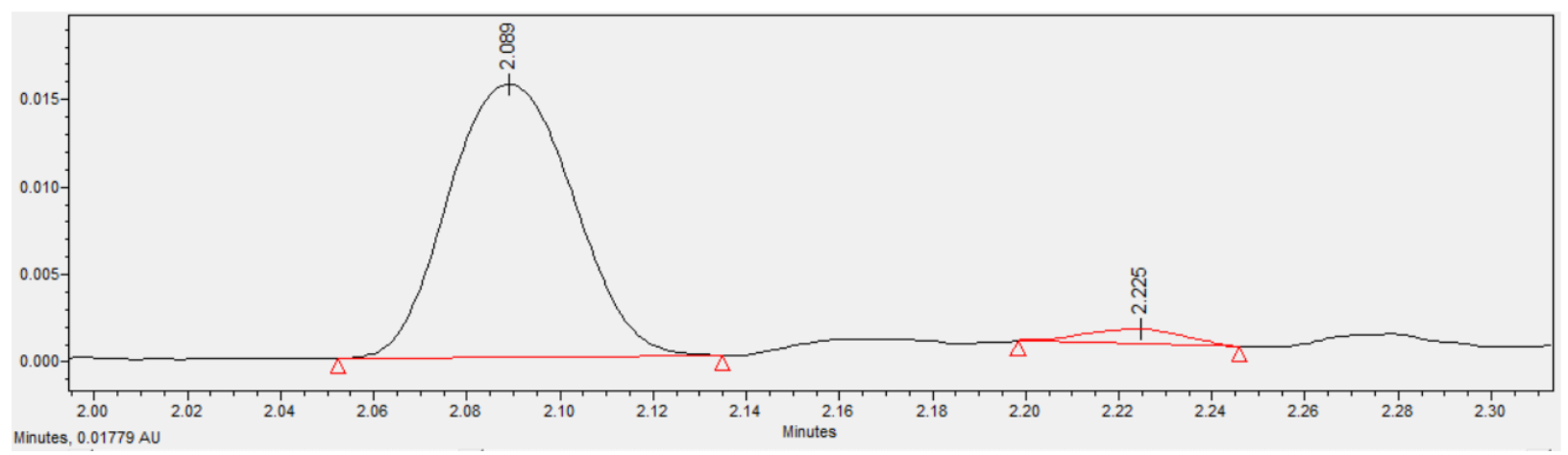

Enantioenriched 16a 


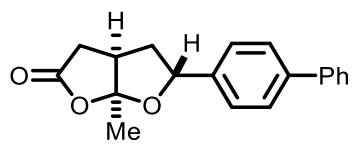

16b: $21.2 \mathrm{mg}$; 48\% (7 h); white solid; purified by column chromatography (hexane/EtOAc = 2/1). ${ }^{1} \mathbf{H}$ NMR $\left(500 \mathrm{MHz}, \mathrm{CDCl}_{3}\right) \delta 7.62-7.54(\mathrm{~m}, 4 \mathrm{H}), 7.47-7.39(\mathrm{~m}, 4 \mathrm{H}), 7.39-7.31(\mathrm{~m}, 1 \mathrm{H}), 5.22(\mathrm{dd}, J=10.4,5.6$ Hz, 1H), $3.08-2.98$ (m, 2H), 2.65 (dd, $J=15.4,7.2 \mathrm{~Hz}, 1 \mathrm{H}), 2.33-2.22(\mathrm{~m}, 1 \mathrm{H}), 2.19$ (ddd, $J=13.4,5.6$, $1.8 \mathrm{~Hz}, 1 \mathrm{H}), 1.81$ (s, 3H). ${ }^{13} \mathbf{C}$ NMR $\left(125 \mathrm{MHz}, \mathrm{CDCl}_{3}\right) \delta 174.8,141.3,140.7,138.1,128.8,127.5,127.4$, 127.1, 126.5, 117.1, 80.1, 43.5, 41.9, 36.6, 24.4. IR (neat, $\left.\mathrm{cm}^{-1}\right): v=3031,2986,2166,1974,1770,1388$, 1256, 1088, 917, 766. HRMS (ESI): $\mathrm{m} / z 295.1329$ calc. for $\mathrm{C}_{19} \mathrm{H}_{19} \mathrm{O}_{3}{ }^{+}[\mathrm{M}+\mathrm{H}]^{+}$, found 295.1328 .

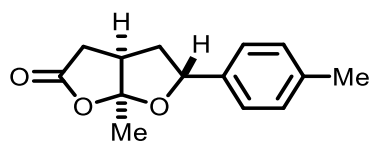

16c: $13.4 \mathrm{mg} ; 50 \%$ (10 h); colorless solid; purified by column chromatography (hexane/EtOAc=2/1). Note: $12.8 \mathrm{mg}$ product $(65 \%, 0.085 \mathrm{mmol}$ scale) was prepared according to the representative procedure with slow addition of DDQ for $1 \mathrm{~h} .{ }^{1} \mathbf{H}$ NMR $\left(500 \mathrm{MHz}, \mathrm{CDCl}_{3}\right) \delta 7.24-7.15(\mathrm{~m}, 4 \mathrm{H}), 5.13(\mathrm{dd}, J=10.4,5.5$ $\mathrm{Hz}, 1 \mathrm{H}), 3.06-2.94(\mathrm{~m}, 2 \mathrm{H}), 2.66-2.56(\mathrm{~m}, 1 \mathrm{H}), 2.35$ (s, 3H), $2.26-2.17(\mathrm{~m}, 1 \mathrm{H}), 2.12$ (ddd, $J=13.0$, 5.5, $1.9 \mathrm{~Hz}, 1 \mathrm{H}), 1.77$ (s, 3H). ${ }^{13} \mathbf{C}$ NMR $\left(125 \mathrm{MHz}, \mathrm{CDCl}_{3}\right) \delta 174.8,138.1,136.1,129.3,126.0,117.1$, 80.2, 43.4, 41.9, 36.6, 24.4, 21.2. IR (neat, $\mathrm{cm}^{-1}$ ): $v=2925,2159,1976,1770,1256,1088$, 919. HRMS (ESI): $m / z 233.1172$ calc. for $\mathrm{C}_{14} \mathrm{H}_{17} \mathrm{O}_{3}{ }^{+}[\mathrm{M}+\mathrm{H}]^{+}$, found 233.1173 .

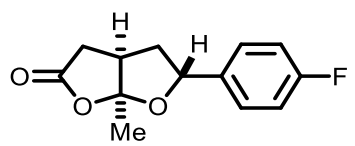

16d: $14.6 \mathrm{mg} ; 44 \%$ (10 h); colorless solid; purified by column chromatography (hexane/EtOAc = 2/1). Note: $14.0 \mathrm{mg}$ product $(62 \%, 0.0985 \mathrm{mmol}$ scale $)$ was prepared according to the representative procedure with slow addition of DDQ for $1 \mathrm{~h} .{ }^{1} \mathbf{H}$ NMR $\left(500 \mathrm{MHz}, \mathrm{CDCl}_{3}\right) \delta 7.35-7.26(\mathrm{~m}, 2 \mathrm{H}), 7.11-7.01(\mathrm{~m}, 2 \mathrm{H})$, $5.13(\mathrm{dd}, J=10.3,5.8 \mathrm{~Hz}, 1 \mathrm{H}), 3.08-2.91(\mathrm{~m}, 2 \mathrm{H}), 2.61(\mathrm{~d}, J=15.4 \mathrm{~Hz}, 1 \mathrm{H}), 2.22-2.07(\mathrm{~m}, 2 \mathrm{H}), 1.77$ (s, 3H). ${ }^{13} \mathbf{C}$ NMR $\left(125 \mathrm{MHz}, \mathrm{CDCl}_{3}\right) \delta 174.7,162.6(\mathrm{~d}, J=246 \mathrm{~Hz}), 134.9(\mathrm{~d}, J=3 \mathrm{~Hz}), 127.7(\mathrm{~d}, J=9$ $\mathrm{Hz}), 117.0,115.5(\mathrm{~d}, J=21 \mathrm{~Hz}), 79.6,43.4,42.0,36.6,24.3 .{ }^{19} \mathbf{F}$ NMR $\left(471 \mathrm{MHz}, \mathrm{CDCl}_{3}\right) \delta-115.0$. IR $\left(\right.$ neat, $\left.\mathrm{cm}^{-1}\right): v=2960,1771,1732,1513,1373,1230,1159,1128,1089,919,836$. HRMS (ESI): $\mathrm{m} / z$ 237.0921 calc. for $\mathrm{C}_{13} \mathrm{H}_{14} \mathrm{FO}_{3}{ }^{+}[\mathrm{M}+\mathrm{H}]^{+}$, found 237.0921 .

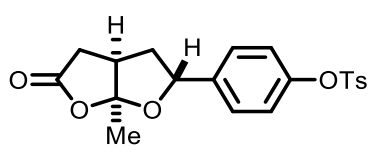


16e: $51.4 \mathrm{mg}$; $68 \%$ (7 h); 55\% with toluene as solvent; colorless solid; purified by column chromatography (hexane/EtOAc = 2/1). ${ }^{1} \mathbf{H}$ NMR $\left(500 \mathrm{MHz}, \mathrm{CDCl}_{3}\right) \delta 7.75-7.56(\mathrm{~m}, 2 \mathrm{H}), 7.33-7.28(\mathrm{~m}, 2 \mathrm{H}), 7.26-$ $7.23(\mathrm{~m}, 2 \mathrm{H}), 7.03-6.93(\mathrm{~m}, 2 \mathrm{H}), 5.10(\mathrm{dd}, J=9.4,6.7 \mathrm{~Hz}, 1 \mathrm{H}), 3.06-2.94(\mathrm{~m}, 2 \mathrm{H}), 2.64-2.53(\mathrm{~m}, 1 \mathrm{H})$, $2.44(\mathrm{~s}, 3 \mathrm{H}), 2.18-2.08(\mathrm{~m}, 2 \mathrm{H}), 1.75$ (s, 3H). ${ }^{13} \mathbf{C} \mathbf{N M R}\left(125 \mathrm{MHz}, \mathrm{CDCl}_{3}\right) \delta 174.6,149.3,145.5,138.2$, 132.3, 129.8, 128.5, 127.2, 122.6, 117.0, 79.4, 43.4, 42.0, 36.5, 24.3, 21.7. IR (neat, $\mathrm{cm}^{-1}$ ): $v=2987,2196$, 1772, 1505, 1371, 1198, 1176, 1154, 1092, 920, 866, 568, 552. HRMS (ESI): $m / z ~ 389.1053$ calc. for $\mathrm{C}_{20} \mathrm{H}_{21} \mathrm{O}_{6} \mathrm{~S}^{+}[\mathrm{M}+\mathrm{H}]^{+}$, found 389.1054 .

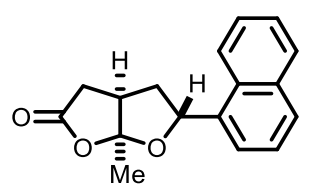

16f: $36.6 \mathrm{mg} ; 67 \%$ (7 h); white solid; purified by column chromatography (hexane/EtOAc = 2/1). ${ }^{1} \mathbf{H}$ NMR $\left(500 \mathrm{MHz}, \mathrm{CDCl}_{3}\right) \delta 8.02-7.72(\mathrm{~m}, 3 \mathrm{H}), 7.69-7.37(\mathrm{~m}, 4 \mathrm{H}), 5.89(\mathrm{t}, J=7.8 \mathrm{~Hz}, 1 \mathrm{H}), 3.13-3.01(\mathrm{~m}$, 2H), $2.76-2.67(\mathrm{~m}, 1 \mathrm{H}), 2.38(\mathrm{ddd}, J=6.9,5.2,1.8 \mathrm{~Hz}, 2 \mathrm{H}), 1.86(\mathrm{~s}, 3 \mathrm{H}) .{ }^{13} \mathbf{C} \mathbf{N M R}\left(125 \mathrm{MHz}, \mathrm{CDCl}_{3}\right)$ $\delta 174.7,134.9,133.7,130.4,128.9,128.6,126.4,125.8,125.4,123.1,122.3,117.1,77.6,43.4,40.7,36.9$, 24.2. IR (neat, $\mathrm{cm}^{-1}$ ): $v=2935,1770,1258,1089,919,836,779$. HRMS (ESI): $\mathrm{m} / z$ 269.1172 calc. for $\mathrm{C}_{17} \mathrm{H}_{17} \mathrm{O}_{3}^{+}[\mathrm{M}+\mathrm{H}]^{+}$, found 269.1172 .

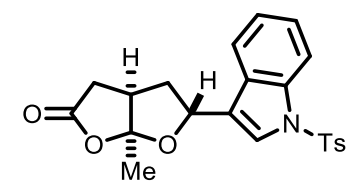

16g: $37.0 \mathrm{mg}$; 45\% (7 h); white solid; purified by column chromatography (hexane/EtOAc = 2/1). ${ }^{1} \mathbf{H}$ NMR $\left(500 \mathrm{MHz}, \mathrm{CDCl}_{3}\right) \delta 7.97(\mathrm{dt}, J=8.4,0.9 \mathrm{~Hz}, 1 \mathrm{H}), 7.80-7.71(\mathrm{~m}, 2 \mathrm{H}), 7.58-7.50(\mathrm{~m}, 2 \mathrm{H}), 7.33(\mathrm{ddd}, J=8.4$, $7.2,1.3 \mathrm{~Hz}, 1 \mathrm{H}), 7.26-7.19(\mathrm{~m}, 3 \mathrm{H}), 5.36(\mathrm{ddd}, J=10.5,5.6,1.0 \mathrm{~Hz}, 1 \mathrm{H}), 3.09-2.98(\mathrm{~m}, 2 \mathrm{H}), 2.69-2.59(\mathrm{~m}$, 1H), $2.51-2.40(\mathrm{~m}, 1 \mathrm{H}), 2.35(\mathrm{~s}, 3 \mathrm{H}), 2.18(\mathrm{ddd}, J=13.2,5.7,2.0 \mathrm{~Hz}, 1 \mathrm{H}), 1.78(\mathrm{~s}, 3 \mathrm{H}) .{ }^{13} \mathbf{C ~ N M R}(125 \mathrm{MHz}$, $\left.\mathrm{CDCl}_{3}\right) \delta 174.5,145.2,135.4,135.1,130.0,128.5,126.9,125.1,123.4,123.2,120.4,120.1,116.9,113.8$, 74.2, 43.3, 39.5, 36.7, 24.3, 21.6. IR (neat, $\mathrm{cm}^{-1}$ ): $v=2925,1771,1446,1370,1258,1174,1123,1087,990$, 917, 582, 538. HRMS (ESI): $m / z 412.1213$ calc. for $\mathrm{C}_{22} \mathrm{H}_{22} \mathrm{NO}_{5} \mathrm{~S}^{+}[\mathrm{M}+\mathrm{H}]^{+}$, found 412.1209 .

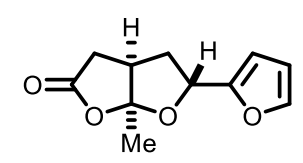

16h: $22.7 \mathrm{mg}$; 55\% (7 h); colorless liquid; purified by column chromatography (hexane/EtOAc = 2/1). ${ }^{1} \mathbf{H}$ NMR $\left(500 \mathrm{MHz}, \mathrm{CDCl}_{3}\right) \delta 7.42(\mathrm{dd}, J=1.8,1.0 \mathrm{~Hz}, 1 \mathrm{H}), 6.40-6.27(\mathrm{~m}, 2 \mathrm{H}), 5.18(\mathrm{dd}, J=9.6,6.0 \mathrm{~Hz}$, 
1H), $3.05-2.93(\mathrm{~m}, 2 \mathrm{H}), 2.65-2.54(\mathrm{~m}, 2 \mathrm{H}), 2.06-1.98(\mathrm{~m}, 1 \mathrm{H}), 1.72(\mathrm{~s}, 3 \mathrm{H}) .{ }^{13} \mathbf{C}$ NMR $(125 \mathrm{MHz}$, $\left.\mathrm{CDCl}_{3}\right) \delta 174.4,151.0,143.2,116.9,110.4,109.1,73.8,43.2,37.3,36.5,24.1$. IR (neat, $\left.\mathrm{cm}^{-1}\right): v=2926$, 1769, 1257, 1088, 999, 916, 745. HRMS (ESI): $m / z 209.0808$ calc. for $\mathrm{C}_{11} \mathrm{H}_{13} \mathrm{O}_{4}{ }^{+}[\mathrm{M}+\mathrm{H}]^{+}$, found 209.0809 .

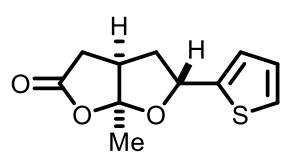

16i: $18.5 \mathrm{mg}$; 42\% (4 h); colorless liquid; purified by column chromatography (hexane/EtOAc $=2 / 1) .{ }^{1} \mathbf{H}$ NMR $\left(500 \mathrm{MHz}, \mathrm{CDCl}_{3}\right) \delta 7.30(\mathrm{dd}, J=5.1,1.2 \mathrm{~Hz}, 1 \mathrm{H}), 7.07-6.94(\mathrm{~m}, 2 \mathrm{H}), 5.42(\mathrm{dd}, J=10.0,5.6 \mathrm{~Hz}$, $1 \mathrm{H}), 3.04-2.94(\mathrm{~m}, 2 \mathrm{H}), 2.64-2.54(\mathrm{~m}, 1 \mathrm{H}), 2.44-2.36(\mathrm{~m}, 1 \mathrm{H}), 2.20(\mathrm{ddd}, J=13.6,5.6,2.2 \mathrm{~Hz}, 1 \mathrm{H})$, $1.75(\mathrm{~s}, 3 \mathrm{H}) .{ }^{13} \mathbf{C}$ NMR $\left(125 \mathrm{MHz}, \mathrm{CDCl}_{3}\right) \delta 174.5,142.1,126.8,125.7,116.8,76.4,43.4,42.0,36.5,24.4$. IR (neat, $\mathrm{cm}^{-1}$ ): $v=2935,1772,1256,1127,1090,919,708$. HRMS (ESI): $\mathrm{m} / \mathrm{z} 225.0580$ calc. for $\mathrm{C}_{11} \mathrm{H}_{13} \mathrm{O}_{3} \mathrm{~S}^{+}[\mathrm{M}+\mathrm{H}]^{+}$, found 225.0581 .

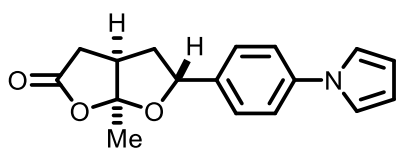

16j: $22.0 \mathrm{mg} ; 40 \%$ (7 h); colorless liquid; purified by column chromatography (hexane/EtOAc $=2 / 1) .{ }^{1} \mathbf{H}$ NMR $\left(500 \mathrm{MHz}, \mathrm{CDCl}_{3}\right) \delta 7.39(\mathrm{~s}, 4 \mathrm{H}), 7.08(\mathrm{t}, J=2.2 \mathrm{~Hz}, 2 \mathrm{H}), 6.35(\mathrm{t}, J=2.2 \mathrm{~Hz}, 2 \mathrm{H}), 5.18(\mathrm{dd}, J=$ 10.4, $5.7 \mathrm{~Hz}, 1 \mathrm{H}), 3.08-2.98(\mathrm{~m}, 2 \mathrm{H}), 2.68-2.59(\mathrm{~m}, 1 \mathrm{H}), 2.27-2.14(\mathrm{~m}, 2 \mathrm{H}), 1.80(\mathrm{~s}, 3 \mathrm{H}) .{ }^{13} \mathbf{C} \mathbf{N M R}$ $\left(125 \mathrm{MHz}, \mathrm{CDCl}_{3}\right) \delta 174.6,140.6,136.4,127.3,120.6,119.3,117.0,110.6,79.7,43.4,41.9,36.6,24.4$. IR (neat, $\mathrm{cm}^{-1}$ ): $v=2935,1769,1524,1328,1256,1124,1088,917,731$. HRMS (ESI): $\mathrm{m} / z$ 284.1281 calc. for $\mathrm{C}_{17} \mathrm{H}_{18} \mathrm{NO}_{3}^{+}[\mathrm{M}+\mathrm{H}]^{+}$, found 284.1281 .

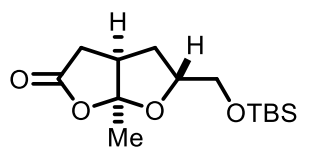

16k: 20.2 mg; 36\% (4 h); colorless liquid; purified by column chromatography (hexane/EtOAc = 2/1). ${ }^{1} \mathbf{H}$ NMR $\left(500 \mathrm{MHz}, \mathrm{CDCl}_{3}\right) \delta 4.28(\mathrm{ddt}, J=7.9,6.5,3.7 \mathrm{~Hz}, 1 \mathrm{H}), 3.74(\mathrm{dd}, J=11.1,3.8 \mathrm{~Hz}, 1 \mathrm{H}), 3.65(\mathrm{dd}, J$ $=11.1,3.6 \mathrm{~Hz}, 1 \mathrm{H}), 2.92-2.78(\mathrm{~m}, 2 \mathrm{H}), 2.51(\mathrm{dd}, J=17.4,1.8 \mathrm{~Hz}, 1 \mathrm{H}), 2.28-2.18(\mathrm{~m}, 1 \mathrm{H}), 1.77(\mathrm{ddd}, J$ $=12.8,6.6,4.1 \mathrm{~Hz}, 1 \mathrm{H}), 1.64(\mathrm{~s}, 3 \mathrm{H}), 0.89(\mathrm{~s}, 9 \mathrm{H}), 0.06(\mathrm{~s}, 6 \mathrm{H}) \cdot{ }^{13} \mathbf{C} \mathbf{~ N M R}\left(125 \mathrm{MHz}, \mathrm{CDCl}_{3}\right) \delta 174.6$, 117.9, 80.1, 64.2, 43.3, 36.8, 34.6, 25.9, 23.8, 18.3, -5.3, -5.4. IR (neat, $\mathrm{cm}^{-1}$ ): $v=2954,2929,2857,1775$, 1253, 1136, 1096, 921, 836, 778. HRMS (ESI): $m / z 287.1673$ calc. for $\mathrm{C}_{14} \mathrm{H}_{27} \mathrm{O}_{4} \mathrm{Si}^{+}[\mathrm{M}+\mathrm{H}]^{+}$, found 287.1674 . 


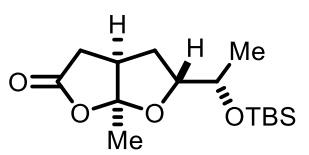

161: $39.1 \mathrm{mg}$; $65 \%$ (6 h); colorless liquid; purified by column chromatography (hexane/EtOAc $=2 / 1) .{ }^{1} \mathbf{H}$ NMR $\left(500 \mathrm{MHz}, \mathrm{CDCl}_{3}\right) \delta 4.03-3.91(\mathrm{~m}, 2 \mathrm{H}), 2.90(\mathrm{dd}, J=18.2,9.6 \mathrm{~Hz}, 1 \mathrm{H}), 2.78(\mathrm{tt}, J=9.7,2.9 \mathrm{~Hz}$, 1H), 2.50 (dd, $J=18.3,2.7 \mathrm{~Hz}, 1 \mathrm{H}), 2.29$ (dt, $J=12.9,9.5 \mathrm{~Hz}, 1 \mathrm{H}), 1.66$ (ddd, $J=12.9,6.0,3.0 \mathrm{~Hz}, 1 \mathrm{H}$ ), $1.62(\mathrm{~s}, 3 \mathrm{H}), 1.09$ (d, $J=6.3 \mathrm{~Hz}, 3 \mathrm{H}), 0.87(\mathrm{~s}, 9 \mathrm{H}), 0.05$ (d, $J=7.9 \mathrm{~Hz}, 6 \mathrm{H}) .{ }^{13} \mathbf{C} \mathbf{N M R}\left(125 \mathrm{MHz}, \mathrm{CDCl}_{3}\right)$ $\delta 174.8,117.6,83.5,68.2,43.0,37.0,32.7,25.8,23.8,20.8,18.0,-4.5,-4.6$. IR (neat, $\mathrm{cm}^{-1}$ ): $v=2955,2930$, 2886, 2857, 1774, 1254, 1091, 1005, 939, 916, 835, 776. HRMS (ESI): $m / z 301.1830$ calc. for $\mathrm{C}_{15} \mathrm{H}_{29} \mathrm{O}_{4} \mathrm{Si}^{+}$ $[\mathrm{M}+\mathrm{H}]^{+}$, found 301.1829 .

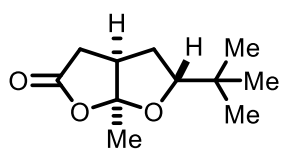

16m: $21.6 \mathrm{mg}$; 55\% (3 h); white solid; purified by column chromatography (hexane/EtOAc $=2 / 1) .{ }^{1} \mathbf{H}$ NMR $\left(500 \mathrm{MHz}, \mathrm{CDCl}_{3}\right) \delta 3.87(\mathrm{dd}, J=10.4,5.8 \mathrm{~Hz}, 1 \mathrm{H}), 2.91(\mathrm{dd}, J=18.4,9.9 \mathrm{~Hz}, 1 \mathrm{H}), 2.77(\mathrm{tt}, J=$ 9.6, $2.7 \mathrm{~Hz}, 1 \mathrm{H}), 2.49$ (dd, $J=18.4,3.2 \mathrm{~Hz}, 1 \mathrm{H}), 2.01(\mathrm{dt}, J=12.9,9.9 \mathrm{~Hz}, 1 \mathrm{H}), 1.62(\mathrm{~s}, 3 \mathrm{H}), 1.62-1.57$ (m, 1H), 0.89 (s, 9H). ${ }^{13} \mathbf{C}$ NMR (125 MHz, $\left.\mathrm{CDCl}_{3}\right) \delta 175.0,117.4,86.7,43.1,37.0,33.8,32.8,25.6,24.1$. IR (neat, $\mathrm{cm}^{-1}$ ): $v=2958,2871,1770,1387,1258,1091,1000$, 917. HRMS (ESI): $\mathrm{m} / z$ 199.1329 calc. for $\mathrm{C}_{11} \mathrm{H}_{19} \mathrm{O}_{3}{ }^{+}[\mathrm{M}+\mathrm{H}]^{+}$, found 199.1329 .

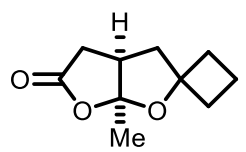

16n: $20.5 \mathrm{mg} ; 57 \%$ (5 h); 46\% with toluene as solvent; colorless liquid; purified by column chromatography (hexane/EtOAc = 2/1). ${ }^{1} \mathbf{H}$ NMR $\left(500 \mathrm{MHz}, \mathrm{CDCl}_{3}\right) \delta 2.94-2.79(\mathrm{~m}, 2 \mathrm{H}), 2.51(\mathrm{dd}, J=17.8,2.1 \mathrm{~Hz}, 1 \mathrm{H})$, $2.44-2.33(\mathrm{~m}, 2 \mathrm{H}), 2.28(\mathrm{dd}, J=13.0,9.2 \mathrm{~Hz}, 1 \mathrm{H}), 2.08-1.97(\mathrm{~m}, 3 \mathrm{H}), 1.77$ (tddd, $J=10.9,6.4,3.2,0.9$ $\mathrm{Hz}, 1 \mathrm{H}), 1.61(\mathrm{~d}, J=0.9 \mathrm{~Hz}, 3 \mathrm{H}), 1.59-1.52(\mathrm{~m}, 1 \mathrm{H}) .{ }^{13} \mathbf{C} \mathbf{N M R}\left(125 \mathrm{MHz}, \mathrm{CDCl}_{3}\right) \delta 174.4,117.3,85.2$, 43.4, 43.3, 37.0, 36.4, 34.7, 24.5, 13.0. IR (neat, $\mathrm{cm}^{-1}$ ): $v=2935,1771,1387,1272,1140,1080,925$. HRMS (ESI): $m / z 183.1016$ calc. for $\mathrm{C}_{10} \mathrm{H}_{15} \mathrm{O}_{3}{ }^{+}[\mathrm{M}+\mathrm{H}]^{+}$, found 183.1016 .

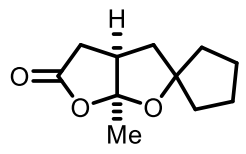


160: $26.6 \mathrm{mg}$; 68\% (3 h); colorless liquid; purified by column chromatography (hexane/EtOAc $=2 / 1) .{ }^{1} \mathbf{H}$ NMR $\left(500 \mathrm{MHz}, \mathrm{CDCl}_{3}\right) \delta 2.93-2.81(\mathrm{~m}, 2 \mathrm{H}), 2.56-2.48(\mathrm{~m}, 1 \mathrm{H}), 2.35-2.27(\mathrm{~m}, 1 \mathrm{H}), 2.02-1.93(\mathrm{~m}$, $1 \mathrm{H}), 1.88-1.71(\mathrm{~m}, 4 \mathrm{H}), 1.65-1.57(\mathrm{~m}, 7 \mathrm{H}) .{ }^{13} \mathbf{C} \mathbf{N M R}\left(125 \mathrm{MHz}, \mathrm{CDCl}_{3}\right) \delta 174.5,117.7,94.6,44.0$, 43.1, 39.6, 39.0, 37.1, 25.0, 24.3, 23.6. IR (neat, $\mathrm{cm}^{-1}$ ): $v=2959,2872,1769,1738,1258,1084,906$. HRMS (ESI): $m / z 197.1172$ calc. for $\mathrm{C}_{11} \mathrm{H}_{17} \mathrm{O}_{3}{ }^{+}[\mathrm{M}+\mathrm{H}]^{+}$, found 197.1173.

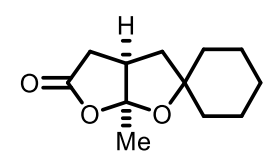

16p: $30.1 \mathrm{mg}$; 72\% (4 h); colorless liquid; purified by column chromatography (hexane/EtOAc $=2 / 1) .{ }^{1} \mathbf{H}$ NMR (500 MHz, $\left.\mathrm{CDCl}_{3}\right) \delta 2.91-2.70(\mathrm{~m}, 2 \mathrm{H}), 2.53-2.43(\mathrm{~m}, 1 \mathrm{H}), 2.27-2.16(\mathrm{~m}, 1 \mathrm{H}), 1.71-1.51(\mathrm{~m}$, 9H), $1.47-1.30(\mathrm{~m}, 5 \mathrm{H}) .{ }^{13} \mathbf{C}$ NMR $\left(125 \mathrm{MHz}, \mathrm{CDCl}_{3}\right) \delta 174.2,117.6,87.2,43.7,42.8,38.7,38.1,37.2$, 25.1, 24.7, 23.4. IR (neat, $\mathrm{cm}^{-1}$ ): $v=2933,2859,1767,1449,1385,1273,1250,1148,983$, 902. HRMS (ESI): $m / z 211.1329$ calc. for $\mathrm{C}_{12} \mathrm{H}_{19} \mathrm{O}_{3}{ }^{+}[\mathrm{M}+\mathrm{H}]^{+}$, found 211.1330 .

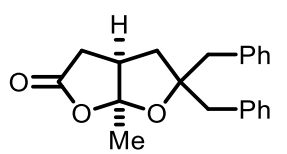

16q: $36.4 \mathrm{mg}$; 57\% (17 h); colorless liquid; purified by column chromatography (hexane/EtOAc $=2 / 1) .{ }^{1} \mathbf{H}$ NMR $\left(500 \mathrm{MHz}, \mathrm{CDCl}_{3}\right) \delta 7.38-7.14(\mathrm{~m}, 10 \mathrm{H}), 3.13(\mathrm{~d}, J=13.7 \mathrm{~Hz}, 1 \mathrm{H}), 2.94(\mathrm{~d}, J=13.7 \mathrm{~Hz}, 1 \mathrm{H}), 2.86$ $(\mathrm{d}, J=13.8 \mathrm{~Hz}, 1 \mathrm{H}), 2.62-2.49(\mathrm{~m}, 2 \mathrm{H}), 2.44-2.36(\mathrm{~m}, 1 \mathrm{H}), 2.33(\mathrm{~d}, J=17.7 \mathrm{~Hz}, 1 \mathrm{H}), 1.77-1.67$ (m, 2H), $1.41(\mathrm{~s}, 3 \mathrm{H}) .{ }^{13} \mathbf{C}$ NMR $\left(125 \mathrm{MHz}, \mathrm{CDCl}_{3}\right) \delta 173.7,136.9,136.8,131.0,130.8,128.1,128.0,126.7$, 126.7, 118.6, 89.8, 48.9, 44.9, 44.2, 40.6, 36.9, 22.8. IR (neat, $\mathrm{cm}^{-1}$ ): $v=3061,3028,2934,1969,1766$, 1454, 1263, 1151, 1093, 895, 751, 702. HRMS (ESI): $m / z 323.1642$ calc. for $\mathrm{C}_{21} \mathrm{H}_{23} \mathrm{O}_{3}{ }^{+}[\mathrm{M}+\mathrm{H}]^{+}$, found 323.1641 .

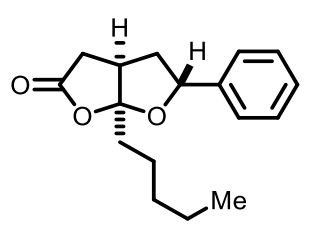

16r: $39.7 \mathrm{mg}$; 73\% (6 h); colorless liquid; purified by column chromatography (hexane/EtOAc $=2 / 1) .{ }^{1} \mathbf{H}$ NMR $\left(500 \mathrm{MHz}, \mathrm{CDCl}_{3}\right) \delta 7.40-7.28(\mathrm{~m}, 5 \mathrm{H}), 5.15(\mathrm{dd}, J=9.6,6.5 \mathrm{~Hz}, 1 \mathrm{H}), 3.08-2.94(\mathrm{~m}, 2 \mathrm{H}), 2.60$ $(\mathrm{dd}, J=18.0,2.7 \mathrm{~Hz}, 1 \mathrm{H}), 2.18-2.10(\mathrm{~m}, 2 \mathrm{H}), 2.04-1.97(\mathrm{~m}, 2 \mathrm{H}), 1.53(\mathrm{pd}, J=5.8,3.2 \mathrm{~Hz}, 2 \mathrm{H}), 1.39-$ $1.31(\mathrm{~m}, J=3.4 \mathrm{~Hz}, 4 \mathrm{H}), 0.91(\mathrm{td}, J=5.8,4.6,2.5 \mathrm{~Hz}, 3 \mathrm{H}) .{ }^{13} \mathbf{C} \mathbf{N M R}\left(125 \mathrm{MHz}, \mathrm{CDCl}_{3}\right) \delta 175.0,139.2$, 128.6, 128.2, 126.0, 119.2, 79.9, 42.3, 41.7, 37.5, 36.7, 31.7, 23.3, 22.5, 14.0. IR (neat, $\left.\mathrm{cm}^{-1}\right): v=2930$, 
2870, 1768, 1251, 1126, 987, 921, 902, 756, 699. HRMS (ESI): $m / z 275.1642$ calc. for $\mathrm{C}_{17} \mathrm{H}_{23} \mathrm{O}_{3}{ }^{+}[\mathrm{M}+\mathrm{H}]^{+}$, found 275.1642 .

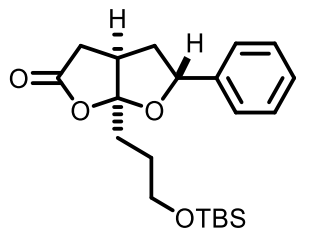

16s: $51.5 \mathrm{mg}$; 68\% (6 h); colorless liquid; purified by column chromatography (hexane/EtOAc $=2 / 1) .{ }^{1} \mathbf{H}$ NMR $\left(500 \mathrm{MHz}, \mathrm{CDCl}_{3}\right) \delta 7.36-7.31(\mathrm{~m}, 5 \mathrm{H}), 5.16(\mathrm{dd}, J=9.9,6.2 \mathrm{~Hz}, 1 \mathrm{H}), 3.74-3.63(\mathrm{~m}, 2 \mathrm{H}), 3.08$ $-2.92(\mathrm{~m}, 2 \mathrm{H}), 2.61(\mathrm{dd}, J=18.2,2.9 \mathrm{~Hz}, 1 \mathrm{H}), 2.19-2.02(\mathrm{~m}, 4 \mathrm{H}), 1.79-1.69(\mathrm{~m}, 2 \mathrm{H}), 0.90(\mathrm{~s}, 9 \mathrm{H}), 0.06$ (s, 6H). ${ }^{13} \mathbf{C}$ NMR $\left(125 \mathrm{MHz}, \mathrm{CDCl}_{3}\right) \delta$ 175.0, 139.2, 128.6, 128.2, 126.0, 119.1, 80.0, 62.6, 42.3, 41.8, 36.6, 34.0, 27.1, 26.0, 18.4, -5.3. IR (neat, $\left.\mathrm{cm}^{-1}\right): v=2955,2929,2856,2032,1974,1776,1257,1099,835$, 777, 699. HRMS (ESI): $m / z 377.2143$ calc. for $\mathrm{C}_{21} \mathrm{H}_{33} \mathrm{O}_{4} \mathrm{Si}^{+}[\mathrm{M}+\mathrm{H}]^{+}$, found 377.2142 .

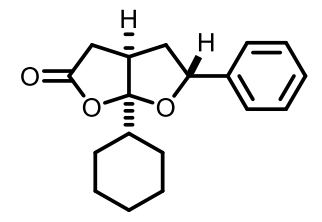

16t: $35.0 \mathrm{mg}$; $60 \%$ (5 h); colorless liquid; purified by column chromatography (hexane/EtOAc $=2 / 1) .{ }^{1} \mathbf{H}$ NMR $\left(500 \mathrm{MHz}, \mathrm{CDCl}_{3}\right) \delta 7.40-7.27(\mathrm{~m}, 5 \mathrm{H}), 5.13(\mathrm{dd}, J=10.6,5.7 \mathrm{~Hz}, 1 \mathrm{H}), 3.11$ (tdd, $J=10.7,3.3$, $1.9 \mathrm{~Hz}, 1 \mathrm{H}), 2.94$ (dd, $J=18.8,10.7 \mathrm{~Hz}, 1 \mathrm{H}), 2.58$ (dd, $J=18.8,3.4 \mathrm{~Hz}, 1 \mathrm{H}), 2.14-2.03$ (m, 2H), $2.00-$ $1.88(\mathrm{~m}, 3 \mathrm{H}), 1.87-1.78(\mathrm{~m}, 2 \mathrm{H}), 1.76-1.69(\mathrm{~m}, 1 \mathrm{H}), 1.31-1.14(\mathrm{~m}, 5 \mathrm{H}) .{ }^{13} \mathbf{C} \mathbf{N M R}\left(125 \mathrm{MHz}, \mathrm{CDCl}_{3}\right)$ $\delta 175.2,139.2,128.6,128.2,126.0,121.0,79.7,44.4,42.7,39.6,36.9,26.9,26.2,25.8$. IR (neat, $\left.\mathrm{cm}^{-1}\right): v$ $=2929,2854,1772,1450,1257,1217,867,918,904,760,699$. HRMS (ESI): $m / z 287.1642$ calc. for $\mathrm{C}_{18} \mathrm{H}_{23} \mathrm{O}_{3}{ }^{+}[\mathrm{M}+\mathrm{H}]^{+}$, found 287.1641.

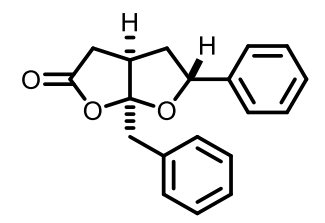

16u: $35.3 \mathrm{mg}$; 60\% (21 h); colorless liquid; purified by column chromatography (hexane/EtOAc $=2 / 1) .{ }^{1} \mathbf{H}$ NMR $\left(500 \mathrm{MHz}, \mathrm{CDCl}_{3}\right) \delta 7.40-7.27$ (m, 10H), $5.16(\mathrm{dd}, J=10.3,5.9 \mathrm{~Hz}, 1 \mathrm{H}), 3.37$ (d, $\left.J=13.9 \mathrm{~Hz}, 1 \mathrm{H}\right)$, 3.29 (d, $J=13.9 \mathrm{~Hz}, 1 \mathrm{H}), 3.08$ (dddd, $J=9.2,6.7,4.2,2.0 \mathrm{~Hz}, 1 \mathrm{H}), 2.52-2.38$ (m, 2H), 2.05 - 1.93 (m, 2H). ${ }^{13} \mathbf{C}$ NMR $\left(125 \mathrm{MHz}, \mathrm{CDCl}_{3}\right) \delta 174.8,139.0,134.3,130.6,128.6,128.3,127.4,126.2,118.2,80.3$, 
43.2, 41.8, 40.9, 36.7. IR (neat, $\left.\mathrm{cm}^{-1}\right): v=3031,2927,1772,1455,1262,983,906,760,700$. HRMS (ESI): $m / z 295.1329$ calc. for $\mathrm{C}_{19} \mathrm{H}_{19} \mathrm{O}_{3}{ }^{+}[\mathrm{M}+\mathrm{H}]^{+}$, found 295.1329 .

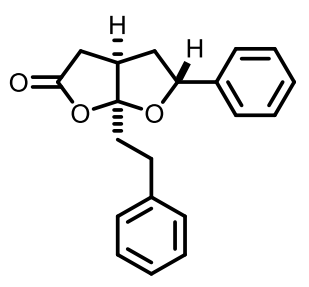

16v: 40.1 mg; 65\% (17 h); colorless liquid; purified by column chromatography (hexane/EtOAc $=2 / 1) .{ }^{1} \mathbf{H}$ NMR (500 MHz, $\left.\mathrm{CDCl}_{3}\right) \delta 7.40-7.29(\mathrm{~m}, 7 \mathrm{H}), 7.28-7.21(\mathrm{~m}, 3 \mathrm{H}), 5.19(\mathrm{dd}, J=9.6,6.5 \mathrm{~Hz}, 1 \mathrm{H}), 3.05$ $-2.87(\mathrm{~m}, 4 \mathrm{H}), 2.68-2.55(\mathrm{~m}, 1 \mathrm{H}), 2.35(\mathrm{q}, J=8.0 \mathrm{~Hz}, 2 \mathrm{H}), 2.22-2.12(\mathrm{~m}, 2 \mathrm{H}) .{ }^{13} \mathbf{C} \mathbf{N M R}(125 \mathrm{MHz}$, $\left.\mathrm{CDCl}_{3}\right) \delta 174.9,140.8,139.0,128.6,128.3,128.3,126.3,126.0,118.4,80.0,42.2,41.9,39.3,36.5,30.0$. IR (neat, $\mathrm{cm}^{-1}$ ): $v=3028,2936,1770,1497,1454,1265,1243,1177,1029,922,902,754,700$. HRMS (ESI): $m / z$ 309.1485 calc. for $\mathrm{C}_{20} \mathrm{H}_{21} \mathrm{O}_{3}{ }^{+}[\mathrm{M}+\mathrm{H}]^{+}$, found 309.1482 .

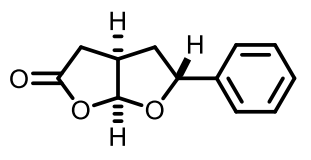

16w: $26.5 \mathrm{mg}$; 65\% (10 h); colorless liquid; purified by column chromatography (hexane/EtOAc $=2 / 1) .{ }^{1} \mathbf{H}$ NMR $\left(500 \mathrm{MHz}, \mathrm{CDCl}_{3}\right) \delta 7.39-7.31(\mathrm{~m}, 5 \mathrm{H}), 6.29(\mathrm{~d}, J=5.6 \mathrm{~Hz}, 1 \mathrm{H}), 5.18(\mathrm{dd}, J=9.8,6.0 \mathrm{~Hz}, 1 \mathrm{H})$, $3.38-3.31(\mathrm{~m}, 1 \mathrm{H}), 2.95(\mathrm{dd}, J=18.8,10.6 \mathrm{~Hz}, 1 \mathrm{H}), 2.56(\mathrm{dd}, J=18.8,4.0 \mathrm{~Hz}, 1 \mathrm{H}), 2.21-2.12(\mathrm{~m}, 2 \mathrm{H})$. ${ }^{13}$ C NMR $\left(125 \mathrm{MHz}, \mathrm{CDCl}_{3}\right) \delta 175.3,138.9,128.6,128.3,126.0,108.1,80.0,41.2,38.9,35.1$. IR (neat, $\left.\mathrm{cm}^{-1}\right): v=2924,2160,1782,1177,1103,975,897,759,701$. HRMS (ESI): $\mathrm{m} / z$ 203.0703 calc. for $\mathrm{C}_{12} \mathrm{H}_{11} \mathrm{O}_{3}{ }^{+}[\mathrm{M}-\mathrm{H}]^{+}$, found 203.0704.

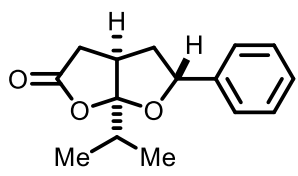

16x: $28.4 \mathrm{mg}$; 58\% (4 h); colorless liquid; purified by column chromatography (hexane/EtOAc = 2/1). ${ }^{1} \mathbf{H}$ NMR $\left(500 \mathrm{MHz}, \mathrm{CDCl}_{3}\right) \delta 7.39-7.29(\mathrm{~m}, 5 \mathrm{H}), 5.20-5.06(\mathrm{~m}, 1 \mathrm{H}), 3.14-3.04(\mathrm{~m}, 1 \mathrm{H}), 2.96(\mathrm{dd}, J=$ $18.8,10.7 \mathrm{~Hz}, 1 \mathrm{H}), 2.60(\mathrm{dd}, J=18.8,3.4 \mathrm{~Hz}, 1 \mathrm{H}), 2.32-2.22(\mathrm{~m}, 1 \mathrm{H}), 2.14-2.03(\mathrm{~m}, 2 \mathrm{H}), 1.11(\mathrm{dd}, J=$ 11.0, 6.8 Hz, 6H). ${ }^{13} \mathbf{C}$ NMR $\left(125 \mathrm{MHz}, \mathrm{CDCl}_{3}\right) \delta 175.3,139.1,128.6,128.2,126.0,121.5,79.8,42.8,39.4$, 37.0, 34.9, 16.9, 16.9. IR (neat, $\mathrm{cm}^{-1}$ ): $v=2969,1769,1245,1227,1098,973,949,923,904,700$. HRMS (ESI): $m / z 247.1329$ calc. for $\mathrm{C}_{15} \mathrm{H}_{19} \mathrm{O}_{3}{ }^{+}[\mathrm{M}+\mathrm{H}]^{+}$, found 247.1327 . 


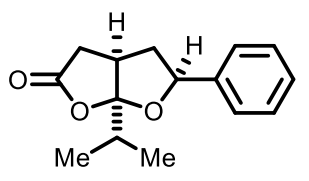

16y: $27.6 \mathrm{mg}$; 56\% (30 h); colorless liquid; purified by column chromatography (hexane/EtOAc $=2 / 1) .{ }^{1} \mathbf{H}$ NMR (500 MHz, $\left.\mathrm{CDCl}_{3}\right) \delta 7.43-7.30(\mathrm{~m}, 4 \mathrm{H}), 7.31-7.25(\mathrm{~m}, 1 \mathrm{H}), 5.17(\mathrm{dd}, J=9.0,6.5 \mathrm{~Hz}, 1 \mathrm{H}), 3.02$ (qd, $J=8.7,1.8 \mathrm{~Hz}, 1 \mathrm{H}), 2.81-2.68(\mathrm{~m}, 2 \mathrm{H}), 2.39(\mathrm{dd}, J=18.2,1.8 \mathrm{~Hz}, 1 \mathrm{H}), 2.19$ (p, $J=6.8 \mathrm{~Hz}, 1 \mathrm{H})$, $1.82(\mathrm{dt}, J=13.0,8.9 \mathrm{~Hz}, 1 \mathrm{H}), 1.08(\mathrm{dd}, J=14.6,6.8 \mathrm{~Hz}, 6 \mathrm{H}) .{ }^{13} \mathbf{C} \mathbf{N M R}\left(125 \mathrm{MHz}, \mathrm{CDCl}_{3}\right) \delta 174.2,141.0$, 128.6, 127.9, 125.6, 121.5, 83.2, 41.8, 40.3, 36.9, 35.3, 16.9, 16.6. IR (neat, $\mathrm{cm}^{-1}$ ): $v=2970,2159,2022$, 1972, 1775, 1061, 998, 945. HRMS (ESI): $m / z 247.1329$ calc. for $\mathrm{C}_{15} \mathrm{H}_{19} \mathrm{O}_{3}{ }^{+}[\mathrm{M}+\mathrm{H}]^{+}$, found 247.1326.

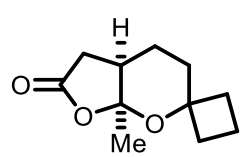

16za: $19.4 \mathrm{mg}$; 50\% (5 h); colorless liquid; purified by column chromatography (hexane/EtOAc $=2 / 1) .{ }^{1} \mathbf{H}$ NMR $\left(500 \mathrm{MHz}, \mathrm{CDCl}_{3}\right) \delta 2.73(\mathrm{dd}, J=17.3,7.4 \mathrm{~Hz}, 1 \mathrm{H}), 2.33(\mathrm{dd}, J=17.3,2.7 \mathrm{~Hz}, 1 \mathrm{H}), 2.30-2.16(\mathrm{~m}$, $3 \mathrm{H}), 2.02-1.90(\mathrm{~m}, 2 \mathrm{H}), 1.89-1.72(\mathrm{~m}, 3 \mathrm{H}), 1.64-1.57(\mathrm{~m}, 2 \mathrm{H}), 1.54-1.49(\mathrm{~m}, 4 \mathrm{H}) .{ }^{13} \mathbf{C}$ NMR $(125$ $\left.\mathrm{MHz}, \mathrm{CDCl}_{3}\right) \delta 174.2,108.7,76.0,37.2,37.1,35.0,34.0,29.6,26.4,22.4,13.2$. IR (neat, $\left.\mathrm{cm}^{-1}\right): v=2988$, 2938, 1770, 1277, 1244, 1111, 1065, 942, 897. HRMS (ESI): $m / z 197.1172$ calc. for $\mathrm{C}_{11} \mathrm{H}_{17} \mathrm{O}_{3}{ }^{+}[\mathrm{M}+\mathrm{H}]^{+}$, found 197.1173 .

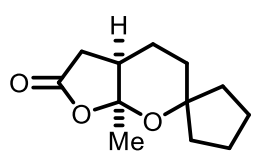

16zb: $20.2 \mathrm{mg}$; 48\% (4 h); white solid; purified by column chromatography (hexane/EtOAc $=2 / 1$ ). ${ }^{1} \mathbf{H}$ NMR $\left(500 \mathrm{MHz}, \mathrm{CDCl}_{3}\right) \delta 2.83(\mathrm{dd}, J=17.4,7.6 \mathrm{~Hz}, 1 \mathrm{H}), 2.31(\mathrm{dd}, J=17.4,1.4 \mathrm{~Hz}, 1 \mathrm{H}), 2.25(\mathrm{dddd}, J$ $=9.9,7.8,6.5,1.4 \mathrm{~Hz}, 1 \mathrm{H}), 2.15-2.07(\mathrm{~m}, 1 \mathrm{H}), 1.90-1.74(\mathrm{~m}, 4 \mathrm{H}), 1.69-1.63(\mathrm{~m}, 1 \mathrm{H}), 1.62-1.56(\mathrm{~m}$, $3 \mathrm{H}), 1.54-1.41(\mathrm{~m}, 6 \mathrm{H}) .{ }^{13} \mathbf{C}$ NMR $\left(125 \mathrm{MHz}, \mathrm{CDCl}_{3}\right) \delta 174.1,109.6,84.9,41.0,37.9,37.3,36.7,31.6$, 27.6, 24.1, 24.0, 22.9. IR (neat, $\mathrm{cm}^{-1}$ ): $v=2941,2870,1767,1250,1179,1117,1022,896,868$. HRMS (ESI): $m / z 211.1329$ calc. for $\mathrm{C}_{12} \mathrm{H}_{19} \mathrm{O}_{3}{ }^{+}[\mathrm{M}+\mathrm{H}]^{+}$, found 211.1328 .

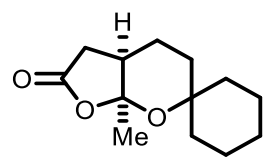

16zc: $23.8 \mathrm{mg}$; 53\% (4 h); white solid; purified by column chromatography (hexane/EtOAc $=2 / 1$ ). ${ }^{1} \mathbf{H}$ NMR $\left(500 \mathrm{MHz}, \mathrm{CDCl}_{3}\right) \delta 2.84(\mathrm{dd}, J=17.6,8.0 \mathrm{~Hz}, 1 \mathrm{H}), 2.35-2.23(\mathrm{~m}, 2 \mathrm{H}), 1.92-1.84(\mathrm{~m}, 1 \mathrm{H}), 1.82$ 
- $1.76(\mathrm{~m}, 1 \mathrm{H}), 1.74-1.64(\mathrm{~m}, 2 \mathrm{H}), 1.64-1.57(\mathrm{~m}, 3 \mathrm{H}), 1.53-1.46(\mathrm{~m}, 5 \mathrm{H}), 1.44-1.27(\mathrm{~m}, 5 \mathrm{H}) .{ }^{13} \mathrm{C}$ NMR $\left(125 \mathrm{MHz}, \mathrm{CDCl}_{3}\right) \delta 174.4,109.6,74.9,39.4,37.8,37.5,35.6,31.0,28.0,25.8,22.4,22.0$. IR (neat, $\left.\mathrm{cm}^{-1}\right): v=2933,2860,1766,1270,1249,1139,1048.896,883$. HRMS (ESI): $\mathrm{m} / z 225.1485$ calc. for $\mathrm{C}_{13} \mathrm{H}_{21} \mathrm{O}_{3}{ }^{+}[\mathrm{M}+\mathrm{H}]^{+}$, found 225.1485 .

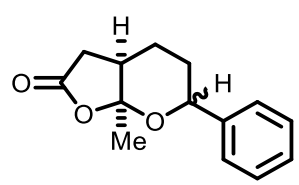

16zd: $35.0 \mathrm{mg}$ as a mixture of $1: 1.3$ diastereomers from a 1:1 mixture of starting materials; $75 \%(4 \mathrm{~h})$; colorless liquid; purified by column chromatography (hexane/EtOAc $=2 / 1) .{ }^{1} \mathbf{H} \mathbf{N M R}\left(500 \mathrm{MHz}, \mathrm{CDCl}_{3}\right)$ $\delta 7.35$ (t, $J=4.4 \mathrm{~Hz}, 9 \mathrm{H}), 7.29$ (dddd, $J=7.1,6.3,3.4,2.0 \mathrm{~Hz}, 2 \mathrm{H}), 4.76$ (dd, $J=11.2,2.3 \mathrm{~Hz}, 1 \mathrm{H}), 4.58$ $(\mathrm{dd}, J=11.0,3.0 \mathrm{~Hz}, 1 \mathrm{H}), 2.90(\mathrm{dd}, J=17.2,6.9 \mathrm{~Hz}, 1 \mathrm{H}), 2.84-2.74(\mathrm{~m}, 1 \mathrm{H}), 2.51-2.43(\mathrm{~m}, 3 \mathrm{H}), 2.40$ - 2.30 (m, 2H), $2.14-2.04$ (m, 3H), $2.01-1.94(\mathrm{~m}, 1 \mathrm{H}), 1.91-1.84(\mathrm{~m}, 1 \mathrm{H}), 1.83-1.51(\mathrm{~m}, 14 \mathrm{H}) .{ }^{13} \mathbf{C}$ NMR $\left(125 \mathrm{MHz}, \mathrm{CDCl}_{3}\right) \delta 175.6,173.9,141.3,141.1,128.5,127.9,127.8,126.1,125.6,109.2,107.0$, 75.6, 74.0, 38.6, 37.7, 36.7, 31.4, 30.4, 27.1, 26.8, 26.4, 22.4, 20.7. IR (neat, $\mathrm{cm}^{-1}$ ): $v=2989,2938,2864$, 1788, 1452, 1385, 1250, 1222, 1176, 1157, 1089, 1075, 1047, 943, 899, 701. HRMS (ESI): $m / z$ 233.1172 calc. for $\mathrm{C}_{14} \mathrm{H}_{17} \mathrm{O}_{3}{ }^{+}[\mathrm{M}+\mathrm{H}]^{+}$, found 233.1172 .

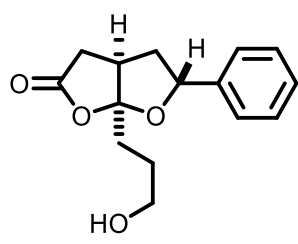

21: $14.0 \mathrm{mg}$; 47\% (5 h); colorless solid; purified by column chromatography (hexane/EtOAc $=2 / 1$ ). ${ }^{1} \mathbf{H}$ NMR $\left(500 \mathrm{MHz}, \mathrm{CDCl}_{3}\right) \delta 7.40-7.29(\mathrm{~m}, 5 \mathrm{H}), 5.17(\mathrm{dd}, J=10.1,6.0 \mathrm{~Hz}, 1 \mathrm{H}), 3.75(\mathrm{td}, J=6.3,1.7 \mathrm{~Hz}$, 2H), $3.09-2.95(\mathrm{~m}, 2 \mathrm{H}), 2.62(\mathrm{dd}, J=18.1,2.7 \mathrm{~Hz}, 1 \mathrm{H}), 2.22-2.08(\mathrm{~m}, 4 \mathrm{H}), 1.84(\mathrm{tt}, J=8.2,6.3 \mathrm{~Hz}$, 2H). ${ }^{13} \mathbf{C}$ NMR $\left(125 \mathrm{MHz}, \mathrm{CDCl}_{3}\right) \delta 174.9,139.0,128.6,128.3,126.0,118.7,80.2,62.4,42.1,42.1,36.6$, 34.1, 26.8. IR (neat, $\left.\mathrm{cm}^{-1}\right): v=3446,2928,2006,1769,1320,1264,1124,1059$, 964, 904, 701. HRMS (ESI): $m / z 285.1097$ calc. for $\mathrm{C}_{15} \mathrm{H}_{18} \mathrm{NaO}_{4}{ }^{+}[\mathrm{M}+\mathrm{Na}]^{+}$, found 285.1096 .

\section{Total Synthesis of Paeonilide}

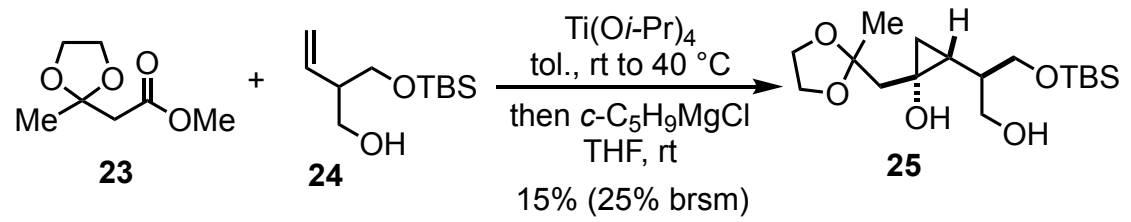


A solution of titanium(IV) isopropoxide ( $0.04 \mathrm{ml}, 0.13 \mathrm{mmol}, 1.1$ equiv.) and alcohol $24^{3}$ (26.4 $\mathrm{mg}, 0.12$ mmol, 1.0 equiv.) in toluene $(0.24 \mathrm{ml})$ was stirred at room temperature for $1 \mathrm{~h}$ and then at $40{ }^{\circ} \mathrm{C}$ (oil bath) for $10 \mathrm{~min}$. After volatile components were removed under vacuum, THF (1.2 mL) and methyl ester $\mathbf{2 3}^{\mathbf{4}}$ (28.8 mg, $0.18 \mathrm{mmol}, 1.5$ equiv.) were added at room temperature, followed by a $2 \mathrm{M}$ solution of cyclopentylmagnesium chloride $(0.31 \mathrm{ml}, 0.61 \mathrm{mmol}, 5.0$ equiv. $)$ in tetrahydrofuran, over a period of $1 \mathrm{~h}$ (with a syringe pump). The reaction mixture was stirred for an additional $14 \mathrm{~h}$ and quenched by addition of water. The resulting mixture was stirred for $1 \mathrm{~h}$, dried over anhydrous sodium sulfate, and filtered. The filter cake was washed with $\mathrm{CH}_{2} \mathrm{Cl}_{2}$, and the combined filtrates were concentrated under reduced pressure. The resulting residue was purified by flash chromatography (pure ether) to give cyclopropanol 25 (4.1 mg, $15 \%, 25 \%$ brsm) as colorless oil.

25: ${ }^{1} \mathbf{H}$ NMR (500 MHz, $\left.\mathrm{CDCl}_{3}\right) \delta 4.00(\mathrm{qd}, J=3.3,1.3 \mathrm{~Hz}, 4 \mathrm{H}), 3.91-3.80(\mathrm{~m}, 3 \mathrm{H}), 3.78-3.72(\mathrm{~m}, 2 \mathrm{H})$, 2.75 (s, 1H), 1.91 (d, $J=2.5 \mathrm{~Hz}, 2 \mathrm{H}), 1.73$ (dddt, $J=8.8,5.3,3.6,1.8 \mathrm{~Hz}, 1 \mathrm{H}), 1.43$ (s, 3H), 0.91 (s, 9H), $0.72(\mathrm{dd}, J=9.5,5.2 \mathrm{~Hz}, 1 \mathrm{H}), 0.64(\mathrm{td}, J=9.8,6.3 \mathrm{~Hz}, 1 \mathrm{H}), 0.51-0.45(\mathrm{~m}, 1 \mathrm{H}), 0.09$ (d, $J=1.9 \mathrm{~Hz}, 6 \mathrm{H})$. ${ }^{13}$ C NMR (125 MHz, $\left.\mathrm{CDCl}_{3}\right) \delta 110.9,67.1,66.5,64.4,55.6,46.4,42.0,25.9,24.4,22.1,18.2,17.3,-5.5$, 5.6. IR (neat, $\mathrm{cm}^{-1}$ ): $v=3462,2953,2929,2884,2857,1472,1380,1253,1146,1093,1051,948,836,778$. HRMS (ESI): $m / z 369.2068$ calc. for $\mathrm{C}_{17} \mathrm{H}_{34} \mathrm{NaO}_{5} \mathrm{Si}^{+}[\mathrm{M}+\mathrm{Na}]^{+}$, found 369.2067.

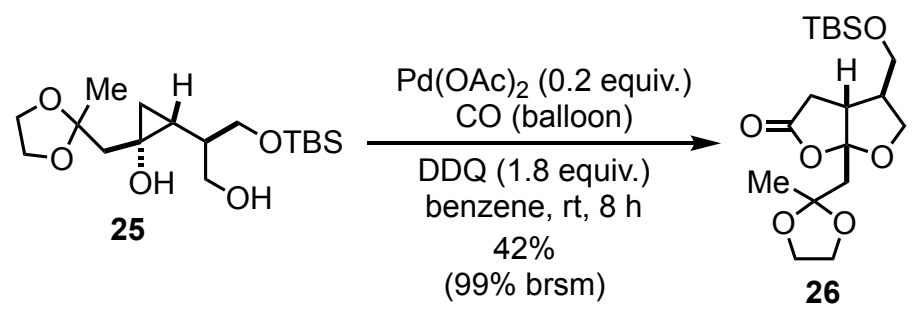

To a stirred solution of 25 (50.0 mg, 0.144 mmol, 1.0 equiv.) and DDQ (59.0 mg, 0.26 mmol, 1.8 equiv.) in benzene $(14.4 \mathrm{ml}, 0.01 \mathrm{M})$ under carbon monoxide (the reactor was evacuated and backfilled three times using a carbon monoxide balloon) was added $\mathrm{Pd}(\mathrm{OAc})_{2}(6.5 \mathrm{mg}, 0.029 \mathrm{mmol}, 0.2$ equiv.) in one portion. The resulting solution was stirred at room temperature for $8 \mathrm{~h}$ before the reaction was quenched with water. The aqueous layer was extracted with ethyl acetate three times. The combined organic layer was washed with brine, dried over $\mathrm{Na}_{2} \mathrm{SO}_{4}$ and concentrated under reduced pressure. The resulting residue was purified by flash chromatography (hexane/ethyl acetate $=2 / 1)$ to obtain $\mathbf{2 6}$ as yellow oil $(22.7 \mathrm{mg}, 42 \%$; $28.5 \mathrm{mg}$ starting material was recovered; 99\% yield brsm).

26: ${ }^{1} \mathbf{H}$ NMR $\left(500 \mathrm{MHz}, \mathrm{CDCl}_{3}\right) \delta 4.05(\mathrm{dd}, J=9.0,7.1 \mathrm{~Hz}, 1 \mathrm{H}), 3.97-3.91(\mathrm{~m}, 4 \mathrm{H}), 3.71-3.58(\mathrm{~m}, 3 \mathrm{H})$, $3.23(\mathrm{ddd}, J=10.4,9.2,3.9 \mathrm{~Hz}, 1 \mathrm{H}), 2.75(\mathrm{dd}, J=18.8,3.9 \mathrm{~Hz}, 1 \mathrm{H}), 2.70-2.57$ (m, 2H), $2.40(\mathrm{~d}, J=15.1$ $\mathrm{Hz}, 1 \mathrm{H}), 2.28(\mathrm{~d}, J=15.1 \mathrm{~Hz}, 1 \mathrm{H}), 1.40(\mathrm{~s}, 3 \mathrm{H}), 0.88(\mathrm{~s}, 9 \mathrm{H}), 0.05(\mathrm{~d}, J=0.7 \mathrm{~Hz}, 6 \mathrm{H}) .{ }^{13} \mathbf{C} \mathbf{N M R}(125$ $\left.\mathrm{MHz}, \mathrm{CDCl}_{3}\right) \delta 175.3,117.3,108.0,68.6,64.4,64.2,60.2,45.2,43.4,43.4,29.9,25.8,25.3,18.1$, -5.5, - 
5.6. IR (neat, $\mathrm{cm}^{-1}$ ): $v=2917,2849,1978,1738,1463,1253,1044,778,697$. HRMS (ESI): $\mathrm{m} / z$ 395.1860 calc. for $\mathrm{C}_{18} \mathrm{H}_{32} \mathrm{NaO}_{6} \mathrm{Si}^{+}[\mathrm{M}+\mathrm{Na}]^{+}$, found 395.1861 .

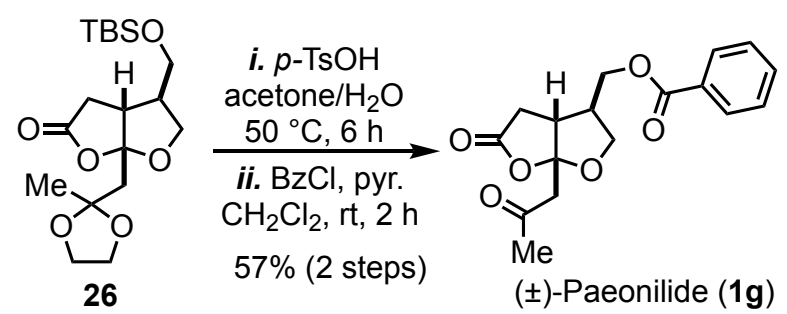

To a solution of $c i s$-fused lactone 26 (13.9 mg, $0.037 \mathrm{mmol}, 1.0$ equiv.) in acetone: $\mathrm{H}_{2} \mathrm{O}=15: 1$ (1.75 ml:0.12 $\mathrm{ml})$ at room temperature was added $\mathrm{TsOH} \cdot \mathrm{H}_{2} \mathrm{O}(3.5 \mathrm{mg}, 0.019 \mathrm{mmol}, 0.5$ equiv. $)$. The reaction was heated at $50{ }^{\circ} \mathrm{C}$ with an oil bath for $6 \mathrm{~h}$. It was then cooled down, quenched with aqueous $\mathrm{NaHCO}_{3}$ and extracted with EtOAc. The combined organic layer was washed with brine, dried with $\mathrm{Na}_{2} \mathrm{SO}_{4}$, and concentrated under reduced pressure. The resulting residue was used in the next step without purification.

To a solution of alcohol $(9.5 \mathrm{mg})$ in $\mathrm{CH}_{2} \mathrm{Cl}_{2}$ at room temperature was added pyridine $(0.03 \mathrm{ml})$ and $\mathrm{BzCl}$ (6.9 $\mu \mathrm{l}, 0.06 \mathrm{mmol}, 2.0$ equiv.). After $2 \mathrm{~h}$, the reaction was concentrated under reduced pressure. The resulting residue was purified by flash chromatography (hexane/ethyl acetate $=2 / 1)$ to obtain $\mathbf{1 g}(6.7 \mathrm{mg}$, $57 \%, 2$ steps) as colorless solid.

${ }^{1} \mathbf{H}$ NMR $\left(500 \mathrm{MHz}, \mathrm{CDCl}_{3}\right) \delta 8.02(\mathrm{~d}, 2 \mathrm{H}, J=7.8 \mathrm{~Hz}), 7.60(\mathrm{t}, 1 \mathrm{H}, J=7.6 \mathrm{~Hz}), 7.47$ (t, 2H, $\left.J=7.6 \mathrm{~Hz}\right), 4.29$ (dd, $1 \mathrm{H}, J=7.2 \mathrm{~Hz}, 11.0 \mathrm{~Hz}), 4.19$ (dd, 1H, $J=8.0 \mathrm{~Hz}, 11.0 \mathrm{~Hz}), 4.00-4.06(\mathrm{~m}, 2 \mathrm{H}), 3.41$ (d, 1H, $J=17.8 \mathrm{~Hz}), 3.35$ (dd, $1 \mathrm{H}, J=10.6 \mathrm{~Hz}, 18.6 \mathrm{~Hz}$ ), 2.94-2.98 (m, 1H), 2.96 (d, 1H, $J=17.6 \mathrm{~Hz}), 2.55$ (dd, 1H, $J=2.8 \mathrm{~Hz}, 18.4 \mathrm{~Hz}), 2.53$ (m, 1H), 2.20 (s, 3H). ${ }^{13} \mathbf{C}$ NMR $\left(125 \mathrm{MHz}, \mathrm{CDCl}_{3}\right) \delta 204.4,174.5,166.4,133.5,129.6,129.5,128.6,115.0$, 67.9, 64.9, 49.5, 46.8, 44.4, 36.6, 31.0. IR (neat, cm-1): $v=2923,2853,1781,1716,1451,1371,1314$, 1271, 1173, 1110, 1071, 1040, 1026, 950, 713. HRMS (ESI): $m / z 319.1176$ calc. for $\mathrm{C}_{17} \mathrm{H}_{19} \mathrm{O}_{6}{ }^{+}[\mathrm{M}+\mathrm{H}]^{+}$, found 319.1177 .

\begin{tabular}{|c|c|c|c|}
\hline \multicolumn{2}{|c|}{${ }^{1} \mathbf{H}$ NMR $\left(500 \mathrm{MHz}, \mathrm{CDCl}_{3}\right)$} & \multicolumn{2}{|c|}{${ }^{13} \mathbf{C}$ NMR $\left(125 \mathrm{MHz}, \mathrm{CDCl}_{3}\right)$} \\
\hline Natural Product & Synthetic Sample & Natural Product & Synthetic Sample \\
\hline $2.20(\mathrm{~s}, 3 \mathrm{H})$ & $2.20(\mathrm{~s}, 3 \mathrm{H})$ & 30.9 & 31.0 \\
\hline $2.54(\mathrm{~m}, 1 \mathrm{H})$ & $2.53(\mathrm{~m}, 1 \mathrm{H})$ & 36.6 & 36.6 \\
\hline $\begin{array}{c}2.55(\mathrm{dd}, 1 \mathrm{H}, \mathrm{J}=2.8 \\
\mathrm{Hz}, 10.5 \mathrm{~Hz})\end{array}$ & $\begin{array}{c}2.55(\mathrm{dd}, 1 \mathrm{H}, \mathrm{J}=2.8 \\
\mathrm{Hz}, 18.4 \mathrm{~Hz})\end{array}$ & 44.4 & 44.4 \\
\hline $\begin{array}{c}2.96(\mathrm{~d}, 1 \mathrm{H}, \mathrm{J}=17.8 \\
\mathrm{Hz})\end{array}$ & $\begin{array}{c}2.96(\mathrm{~d}, 1 \mathrm{H}, \mathrm{J}=17.6 \\
\mathrm{Hz})\end{array}$ & 46.7 & 46.8 \\
\hline $2.97(\mathrm{~m}, 1 \mathrm{H})$ & $2.94-2.98(\mathrm{~m}, 1 \mathrm{H})$ & 49.5 & 49.5 \\
\hline $\begin{array}{c}3.34(\mathrm{dd}, 1 \mathrm{H}, \mathrm{J}=8.0 \\
\mathrm{Hz}, 10.5 \mathrm{~Hz})\end{array}$ & $\begin{array}{c}3.35(\mathrm{dd}, 1 \mathrm{H}, \\
J=10.6 \mathrm{~Hz}, 18.6 \mathrm{~Hz})\end{array}$ & 64.9 & 64.9 \\
\hline $\begin{array}{c}3.40(\mathrm{~d}, 1 \mathrm{H}, J=17.8 \\
\mathrm{Hz})\end{array}$ & $\begin{array}{c}3.41(\mathrm{~d}, 1 \mathrm{H}, \mathrm{J}=17.8 \\
\mathrm{Hz})\end{array}$ & 67.9 & 67.9 \\
\hline
\end{tabular}




\begin{tabular}{|c|c|c|c|}
\hline $4.03(\mathrm{~m}, 2 \mathrm{H})$ & $4.00-4.06(\mathrm{~m}, 2 \mathrm{H})$ & 114.9 & 115.0 \\
\hline $\begin{array}{c}4.19(\mathrm{dd}, 1 \mathrm{H}, J=8.0 \\
\mathrm{Hz}, 11.0 \mathrm{~Hz})\end{array}$ & $\begin{array}{c}4.19(\mathrm{dd}, 1 \mathrm{H}, J=8.0 \\
\mathrm{Hz}, 11.0 \mathrm{~Hz})\end{array}$ & 128.5 & 128.6 \\
\hline $\begin{array}{c}4.30(\mathrm{dd}, 1 \mathrm{H}, J=7.3 \\
\mathrm{Hz}, 11.0 \mathrm{~Hz})\end{array}$ & $\begin{array}{c}4.29(\mathrm{dd}, 1 \mathrm{H}, J=7.2 \\
\mathrm{Hz}, 11.0 \mathrm{~Hz})\end{array}$ & 129.6 & 129.6 \\
\hline $\begin{array}{c}7.47(\mathrm{t}, 2 \mathrm{H}, \mathrm{J}=8.5 \\
\mathrm{Hz})\end{array}$ & $\begin{array}{c}7.47(\mathrm{t}, 2 \mathrm{H}, J=7.6 \\
\mathrm{Hz})\end{array}$ & 129.6 & 133.5 \\
\hline $7.60(\mathrm{brt.}, 1 \mathrm{H})$ & $\begin{array}{c}7.60(\mathrm{t}, 1 \mathrm{H}, J=7.6 \\
\mathrm{Hz})\end{array}$ & 166.3 & 166.4 \\
\hline $\begin{array}{c}8.02(\mathrm{dd}, 2 \mathrm{H}, J=1.2 \\
\mathrm{Hz}, 8.5 \mathrm{~Hz})\end{array}$ & $\begin{array}{c}8.02(\mathrm{~d}, 2 \mathrm{H}, J=7.8 \\
\mathrm{Hz})\end{array}$ & 174.4 & 174.5 \\
\hline & & 207.1 & 204.4 \\
\hline
\end{tabular}

\section{Diversification}

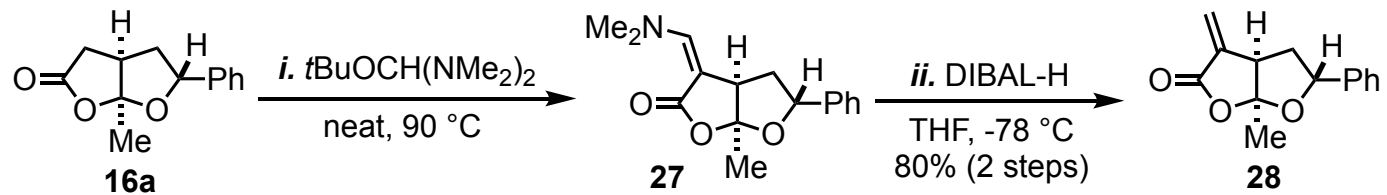

To a solution of cis-fused lactone $16 \mathrm{a}(55 \mathrm{mg}, 0.252 \mathrm{mmol}, 1.0$ equiv.) was added Bredereck's reagent (219.6 mg, $1.26 \mathrm{mmol}, 5.0$ equiv.). The reaction was heated to $90{ }^{\circ} \mathrm{C}$ using an oil bath. After $4 \mathrm{~h}$, the reaction was cooled to room temperature, the crude was purified by flash column $(\mathrm{Hex} / \mathrm{EA}=1 / 3)$ directly without work-up to give $66 \mathrm{mg}$ crude product.

To a solution of the above crude enamine $(61.5 \mathrm{mg}, 0.225 \mathrm{mmol}, 1.0$ equiv.) in THF ( $4.5 \mathrm{ml}, 0.05 \mathrm{M})$ at $78^{\circ} \mathrm{C}$ was added DIBAL-H (2.4 equiv.) dropwise. After $1 \mathrm{~h}$, the reaction was quenched with a saturated aqueous solution of $\mathrm{NH}_{4} \mathrm{Cl}$, extracted with EtOAc, washed with brine, dried with $\mathrm{Na}_{2} \mathrm{SO}_{4}$, and concentrated. The crude residue was purified by flash column $(\mathrm{Hex} / \mathrm{EA}=5 / 1)$ to give $\alpha$-methylene $\gamma$-butyrolactone 28 (42.8 mg, $80 \%$, over 2 steps) as white solid.

28: ${ }^{1} \mathrm{H}$ NMR $\left(500 \mathrm{MHz}, \mathrm{CDCl}_{3}\right) \delta 7.40-7.28(\mathrm{~m}, 5 \mathrm{H}), 6.42(\mathrm{~d}, J=2.6 \mathrm{~Hz}, 1 \mathrm{H}), 5.78(\mathrm{~d}, J=2.2 \mathrm{~Hz}, 1 \mathrm{H})$, $5.03(\mathrm{dd}, J=11.1,5.0 \mathrm{~Hz}, 1 \mathrm{H}), 3.49(\mathrm{dd}, J=8.8,1.8 \mathrm{~Hz}, 1 \mathrm{H}), 2.39-2.21(\mathrm{~m}, 2 \mathrm{H}), 1.79(\mathrm{~s}, 3 \mathrm{H}) .{ }^{13} \mathbf{C} \mathbf{N M R}$ $\left(125 \mathrm{MHz}, \mathrm{CDCl}_{3}\right) \delta 168.7,139.6,138.7,128.6,128.3,126.0,124.4,113.9,80.3,48.7,43.6,24.6$. IR (neat, $\left.\mathrm{cm}^{-1}\right): v=2937,1762,1276,1125,1067,927,913,872,759,700$. HRMS (ESI): $\mathrm{m} / z 231.1016$ calc. for $\mathrm{C}_{14} \mathrm{H}_{15} \mathrm{O}_{3}^{+}[\mathrm{M}+\mathrm{H}]^{+}$, found 231.1014 .

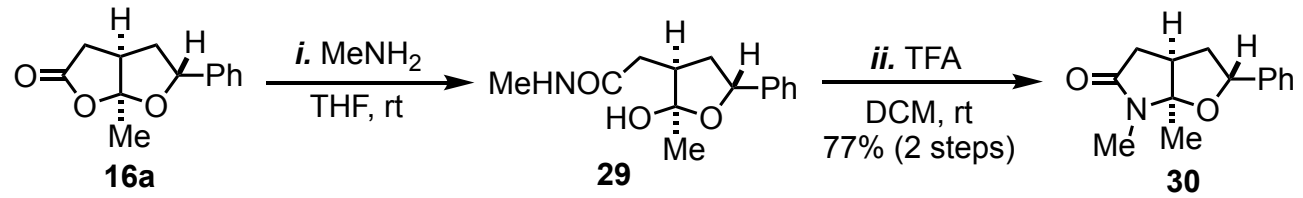


A solution of $\mathrm{MeNH}_{2}(64.0 \mathrm{mg}, 40 \mathrm{wt} \%$ in water, $2.06 \mathrm{mmol}, 15.0$ equiv) was added to cis-fused lactone 16a (30.0 mg, $0.138 \mathrm{mmol}, 1.0$ equiv.) in THF $(1.4 \mathrm{ml}, 0.1 \mathrm{M})$ at room temperature. The reaction mixture was stirred at room temperature for $1 \mathrm{~h}$. The resulting mixture was evaporated to dry under reduced pressure. The crude is proceeded to the next step without purification.

To a solution of the above crude $(0.138 \mathrm{mmol}, 1.0$ equiv. $)$ in DCM $(1.4 \mathrm{ml}, 0.1 \mathrm{M})$ was added TFA $(21 \mu 1$, $0.275 \mathrm{mmol}, 2.0$ equiv.) in one portion at room temperature. After the reaction was stirred at room temperature for $30 \mathrm{~min}$, it was quenched with a saturated aqueous solution of $\mathrm{NH}_{4} \mathrm{Cl}$, extracted with EtOAc, washed with brine, dried with $\mathrm{Na}_{2} \mathrm{SO}_{4}$, and concentrated. The crude was purified by flash column (Hex/EA $=6 / 1)$ to give lactam $30(24.5 \mathrm{mg}, 77 \%$, over 2 steps $)$ as colorless liquid.

30: ${ }^{1} \mathrm{H}$ NMR $\left(500 \mathrm{MHz}, \mathrm{CDCl}_{3}\right) \delta 7.43-7.26(\mathrm{~m}, 5 \mathrm{H}), 4.78(\mathrm{dd}, J=10.9,5.0 \mathrm{~Hz}, 1 \mathrm{H}), 2.84(\mathrm{~s}, 3 \mathrm{H}), 2.80$ $(\mathrm{d}, J=6.5 \mathrm{~Hz}, 2 \mathrm{H}), 2.39$ (d, $J=14.7 \mathrm{~Hz}, 1 \mathrm{H}), 2.21-2.11(\mathrm{~m}, 1 \mathrm{H}), 2.03(\mathrm{dd}, J=12.6,5.0 \mathrm{~Hz}, 1 \mathrm{H}), 1.64$ (s, 3H). ${ }^{13} \mathrm{C}$ NMR $\left(125 \mathrm{MHz}, \mathrm{CDCl}_{3}\right) \delta 139.7,128.6,128.1,126.1,100.6,79.0,43.3,41.5,37.4,24.6,23.4$. IR (neat, $\left.\mathrm{cm}^{-1}\right): v=2939,1674,1602,1423,1204,1149,1131,1066,801,756,723,699$. HRMS (ESI): $m / z 232.1332$ calc. for $\mathrm{C}_{14} \mathrm{H}_{18} \mathrm{NO}_{2}{ }^{+}[\mathrm{M}+\mathrm{H}]^{+}$, found 232.1331 .

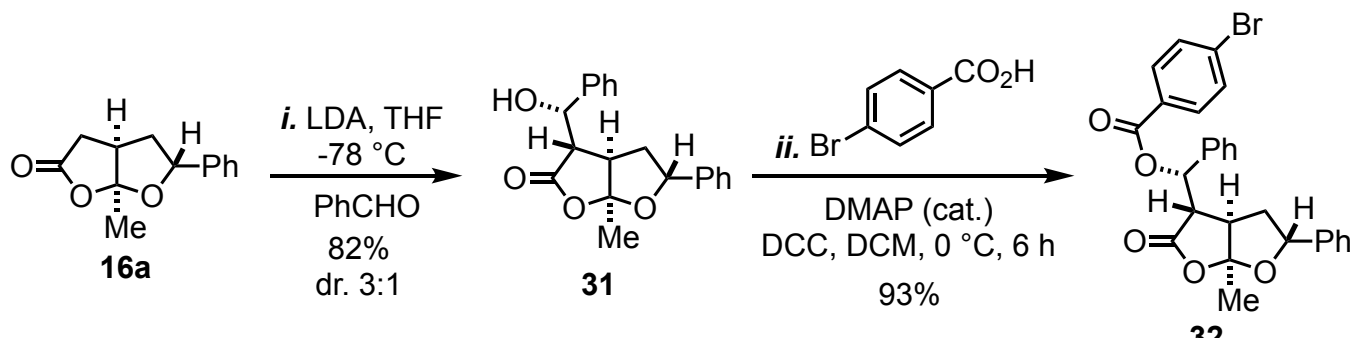

To a solution of cis-fused lactone $\mathbf{1 6 a}(26.4 \mathrm{mg}, 0.121 \mathrm{mmol}, 1.0$ equiv. $)$ in THF $(1.2 \mathrm{ml}, 0.1 \mathrm{M})$ at $-78{ }^{\circ} \mathrm{C}$ was added $1.0 \mathrm{M}$ LDA $(0.145 \mathrm{ml}, 0.145 \mathrm{mmol}, 1.2$ equiv. $)$ dropwise. After $30 \mathrm{~min}$, benzaldehyde $(15 \mu \mathrm{l}$, $0.145 \mathrm{mmol}, 1.2$ equiv.) was added dropwise at $-78^{\circ} \mathrm{C}$ and the reaction was stirred at this temperature for $1.5 \mathrm{~h}$. The reaction was quenched with a saturated aqueous solution of $\mathrm{NH}_{4} \mathrm{Cl}$ and extracted with EtOAc. The combined organic layers were washed with brine and evaporated. The residue was purified via flash column (Hex:EA = 5:1) to give aldol product 31 (dr. 3:1, separable, total $32.0 \mathrm{mg}, 82 \%)$ as white solid.

31, major diastereomer: ${ }^{1} \mathbf{H}$ NMR $\left(500 \mathrm{MHz}, \mathrm{CDCl}_{3}\right) \delta 7.43-7.36(\mathrm{~m}, 4 \mathrm{H}), 7.32-7.24(\mathrm{~m}, 6 \mathrm{H}), 5.49$ $(\mathrm{dd}, J=4.8,2.4 \mathrm{~Hz}, 1 \mathrm{H}), 5.03(\mathrm{dd}, J=11.5,4.5 \mathrm{~Hz}, 1 \mathrm{H}), 3.10-2.97(\mathrm{~m}, 2 \mathrm{H}), 2.70(\mathrm{~d}, J=4.7 \mathrm{~Hz}, 1 \mathrm{H})$, $2.00-1.87(\mathrm{~m}, 1 \mathrm{H}), 1.73(\mathrm{~s}, 3 \mathrm{H}), 1.61(\mathrm{dd}, J=12.9,4.6 \mathrm{~Hz}, 1 \mathrm{H}) .{ }^{13} \mathbf{C} \mathbf{N M R}\left(125 \mathrm{MHz}, \mathrm{CDCl}_{3}\right) \delta 176.3$, 141.0, 138.8, 128.8, 128.5, 128.2, 128.0, 126.0, 125.3, 116.4, 80.4, 71.6, 56.9, 43.7, 42.1, 24.5. IR (neat, $\left.\mathrm{cm}^{-1}\right): v=3478,3033,2934,1759,1453,1389,1266,1128,1091,905,760,700$. HRMS (ESI): $\mathrm{m} / z$ 347.1254 calc. for $\mathrm{C}_{20} \mathrm{H}_{20} \mathrm{NaO}_{4}{ }^{+}[\mathrm{M}+\mathrm{Na}]^{+}$, found 347.1250 . 
31a, minor diastereomer: ${ }^{1} \mathbf{H}$ NMR $\left(500 \mathrm{MHz}, \mathrm{CDCl}_{3}\right) \delta 7.47-7.36(\mathrm{~m}, 4 \mathrm{H}), 7.36-7.26(\mathrm{~m}, 5 \mathrm{H}), 7.25$ (s, 1H), 5.07 (td, $J=10.0,8.7,2.9 \mathrm{~Hz}, 2 \mathrm{H}), 3.65$ (d, $J=1.7 \mathrm{~Hz}, 1 \mathrm{H}), 3.10$ (dd, $J=7.8,5.6 \mathrm{~Hz}, 1 \mathrm{H}), 2.70$ (dd, $J=8.4,5.5 \mathrm{~Hz}, 1 \mathrm{H}), 1.96(\mathrm{ddd}, J=13.0,11.4,8.3 \mathrm{~Hz}, 1 \mathrm{H}), 1.77$ (dd, $J=13.0,4.6 \mathrm{~Hz}, 1 \mathrm{H}), 1.45$ (s, $3 \mathrm{H}) .{ }^{13} \mathrm{C}$ NMR $\left(125 \mathrm{MHz}, \mathrm{CDCl}_{3}\right) \delta 176.6,139.6,138.6,128.9,128.8,128.6,128.3,126.7,126.0,116.0$, 80.6, 74.4, 55.3, 46.3, 41.7, 24.8. IR (neat, $\mathrm{cm}^{-1}$ ): $v=3478,3033,2934,1759,1453,1389,1266,1128$, 1091, 905, 760, 700. HRMS (ESI): $m / z 347.1254$ calc. for $\mathrm{C}_{20} \mathrm{H}_{20} \mathrm{NaO}_{4}{ }^{+}[\mathrm{M}+\mathrm{Na}]^{+}$, found 347.1250 .

To a mixture of the major diastereomer of aldol product 31 (30.0 mg, $0.093 \mathrm{mmol}, 1.0$ equiv.), DMAP (1.0 mg), 4-bromo benzoic acid (20.5 mg, $0.102 \mathrm{mmol}, 1.1$ equiv.) was added $\mathrm{CH}_{2} \mathrm{Cl}_{2}(0.93 \mathrm{ml}, 0.1 \mathrm{M})$. DCC ( $22.0 \mathrm{mg}, 0.107 \mathrm{mmol}, 1.15$ equiv.) were sequentially added to the solution at $0{ }^{\circ} \mathrm{C}$. The resulting mixture was stirred at $0{ }^{\circ} \mathrm{C}$ for $6 \mathrm{~h}$ before it was quenched with water. The layers were separated, and the aqueous layer was extracted with $\mathrm{CH}_{2} \mathrm{Cl}_{2}$. The combined organic layers were dried with $\mathrm{Na}_{2} \mathrm{SO}_{4}$ and concentrated under reduced pressure. The residue was purified by flash column $(\mathrm{Hex} / \mathrm{EA}=8 / 1)$ to give $32(43.5 \mathrm{mg}$, $93 \%$ ) as white solid.

32: ${ }^{1} \mathbf{H}$ NMR (500 MHz, $\left.\mathrm{CDCl}_{3}\right) \delta 7.99-7.87(\mathrm{~m}, 2 \mathrm{H}), 7.68-7.59(\mathrm{~m}, 2 \mathrm{H}), 7.42-7.26(\mathrm{~m}, 10 \mathrm{H}), 6.55(\mathrm{~d}$, $J=3.5 \mathrm{~Hz}, 1 \mathrm{H}), 5.07(\mathrm{dd}, J=11.4,4.4 \mathrm{~Hz}, 1 \mathrm{H}), 3.29(\mathrm{dd}, J=5.7,3.5 \mathrm{~Hz}, 1 \mathrm{H}), 3.17$ (dd, $J=8.2,5.8 \mathrm{~Hz}$, $1 \mathrm{H}), 2.05(\mathrm{ddd}, J=12.9,11.4,8.3 \mathrm{~Hz}, 1 \mathrm{H}), 1.77(\mathrm{dd}, J=13.0,4.5 \mathrm{~Hz}, 1 \mathrm{H}), 1.66(\mathrm{~s}, 3 \mathrm{H}) .{ }^{13} \mathbf{C}$ NMR $(125$ $\left.\mathrm{MHz}, \mathrm{CDCl}_{3}\right) \delta 173.6,164.3,138.4,137.0,132.0,131.1,129.1,128.8,128.6,128.4,128.4,126.0,125.7$, 115.4, 80.4, 74.2, 54.6, 45.2, 42.1, 25.1. IR (neat, $\left.\mathrm{cm}^{-1}\right): v=2243,2072,1974,1771,1728,1590,1300$, 1266, 1092, 1012, 755, 699. HRMS (ESI): $m / z$ 529.0621/531.0601 calc. for $\mathrm{C} 27 \mathrm{H} 23 \mathrm{BrNaO}^{+}[\mathrm{M}+\mathrm{Na}]^{+}$, found $529.0620 / 531.0603$.

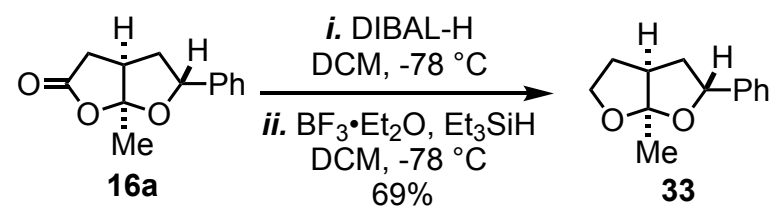

To a solution of cis-fused lactone $16 \mathbf{a}\left(41.5 \mathrm{mg}, 0.19 \mathrm{mmol}, 1.0\right.$ equiv.) in anhydrous $\mathrm{CH}_{2} \mathrm{Cl}_{2}(1.9 \mathrm{ml}, 0.1$ M) was added 1.0 M DIBAL-H solution in toluene $(0.21 \mathrm{~mL}, 0.21 \mathrm{mmol}, 1.1$ equiv. $)$ at $-78^{\circ} \mathrm{C}$. The reaction mixture was stirred for $30 \mathrm{~min}$ and the progress of the reaction was monitored by TLC. After the reaction was over, a saturated solution of sodium potassium tartrate was added. The reaction was allowed to stir for 4-6 $\mathrm{h}$ till a clear separation of organic and aqueous layers resulted. The organic layer was separated and aqueous layer was further extracted with $\mathrm{CH}_{2} \mathrm{Cl}_{2}$. The combined organic layers were dried over $\mathrm{Na}_{2} \mathrm{SO}_{4}$ and evaporated under vacuum. The residue obtained was purified by column chromatography $(\mathrm{Hex} / \mathrm{EA}=1 / 1)$ to yield a hemiacetal (43.3 mg, quant.) as colorless oil. 
To a solution of the above hemiacetal $\left(15.9 \mathrm{mg}, 0.723 \mathrm{mmol}, 1.0\right.$ equiv.) in anhydrous $\mathrm{CH}_{2} \mathrm{Cl}_{2}(5 \mathrm{~mL})$ at $0{ }^{\circ} \mathrm{C}$, triethylsilane ( $35 \mu 1,0.217 \mathrm{mmol}, 3.0$ equiv.) was added and the reaction mixture was cooled to $78{ }^{\circ} \mathrm{C}$. A solution of $\mathrm{BF}_{3}$ etherate $(18 \mu 1,0.145 \mathrm{mmol}, 2.0$ equiv.) was added slowly to the reaction mixture and the reaction was allowed to raise to room temperature. After completion, a saturated solution of sodium bicarbonate was added. The mixture was extracted with $\mathrm{CH}_{2} \mathrm{Cl}_{2}$. The combined organic layers were washed with brine, dried over $\mathrm{Na}_{2} \mathrm{SO}_{4}$. The organic solvent was removed by rotatory evaporation. The residue was purified by column chromatography on silica gel with $(\mathrm{Hex} / \mathrm{EA}=2: 1)$ to yield $33(10.1 \mathrm{mg}, 69 \%)$ as colorless oil.

33: ${ }^{1} \mathbf{H}$ NMR ${ }^{1} \mathrm{H}$ NMR $\left(500 \mathrm{MHz}, \mathrm{CDCl}_{3}\right) \delta 7.35-7.26(\mathrm{~m}, 5 \mathrm{H}), 5.16(\mathrm{dd}, J=10.6,5.6 \mathrm{~Hz}, 1 \mathrm{H}), 4.09$ (td, $J=8.6,6.7 \mathrm{~Hz}, 1 \mathrm{H}), 3.94(\mathrm{ddd}, J=9.0,7.9,4.4 \mathrm{~Hz}, 1 \mathrm{H}), 2.69$ (d, $J=4.1 \mathrm{~Hz}, 1 \mathrm{H}), 2.34-2.25(\mathrm{~m}, 1 \mathrm{H})$, $2.16(\mathrm{ddd}, J=12.8,5.6,1.7 \mathrm{~Hz}, 1 \mathrm{H}), 2.12-2.05(\mathrm{~m}, 1 \mathrm{H}), 1.91-1.85(\mathrm{~m}, 1 \mathrm{H}), 1.63(\mathrm{~s}, 3 \mathrm{H}) .{ }^{13} \mathbf{C} \mathbf{N M R}(125$ $\left.\mathrm{MHz}, \mathrm{CDCl}_{3}\right) \delta 141.8,128.4,127.5,125.8,116.7,81.0,68.2,47.1,42.4,33.1,24.5$. IR (neat, $\left.\mathrm{cm}^{-1}\right): v=$ 2926, 2854, 1452, 1380, 1108, 1016, 755, 699. HRMS (ESI): $m / z 203.1068$ calc. for $\mathrm{C}_{13} \mathrm{H}_{15} \mathrm{O}_{2}{ }^{+}[\mathrm{M}-\mathrm{H}]^{+}$, found 203.1067 .

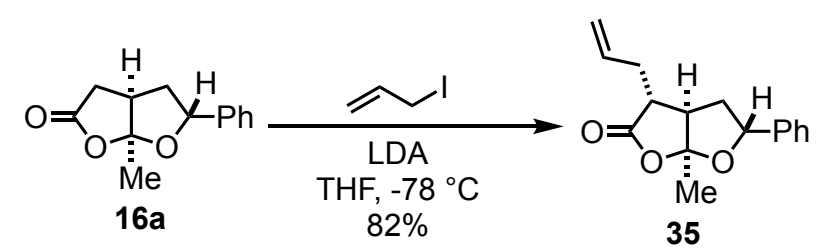

To a solution of $c i s$-fused lactone $16 \mathrm{a}\left(24.0 \mathrm{mg}, 0.11 \mathrm{mmol}, 1.0\right.$ equiv.) in THF $(0.1 \mathrm{M})$ at $-78{ }^{\circ} \mathrm{C}$ was added 1.0 M LDA (0.22 mmol, 2.0 equiv.) dropwise. After $1 \mathrm{~h}$, allyl iodide ( $25 \mu \mathrm{l}, 0.275 \mathrm{mmol}, 2.5$ equiv.) was added over $5 \mathrm{~min}$ at $-78{ }^{\circ} \mathrm{C}$ and the reaction was stirred at this temperature for $1 \mathrm{~h}$ before it was quenched with a saturated solution of $\mathrm{NH}_{4} \mathrm{Cl}$ and extracted with EtOAc. The combined organic layer was washed with brine and evaporated. The residue was purified via flash column $(\mathrm{Hex} / \mathrm{EA}=3 / 1)$ to give $35(23.4 \mathrm{mg}$, $82 \%)$ as colorless liquid.

35: ${ }^{1} \mathbf{H}$ NMR $\left(500 \mathrm{MHz}, \mathrm{CDCl}_{3}\right) \delta 7.40-7.29(\mathrm{~m}, 5 \mathrm{H}), 5.85-5.71(\mathrm{~m}, 1 \mathrm{H}), 5.24-5.14(\mathrm{~m}, 2 \mathrm{H}), 5.12(\mathrm{dd}$, $J=10.6,5.3 \mathrm{~Hz}, 1 \mathrm{H}), 2.84-2.70(\mathrm{~m}, 2 \mathrm{H}), 2.70-2.59(\mathrm{~m}, 1 \mathrm{H}), 2.48-2.39(\mathrm{~m}, 1 \mathrm{H}), 2.24-2.09(\mathrm{~m}, 2 \mathrm{H})$, $1.76(\mathrm{~s}, 3 \mathrm{H}) .{ }^{13} \mathrm{C}$ NMR $\left(125 \mathrm{MHz}, \mathrm{CDCl}_{3}\right) \delta 176.9,139.0,133.8,128.6,128.3,126.0,118.9,115.4,80.5$, 48.7, 48.0, 42.0, 36.0, 25.4. IR (neat, $\mathrm{cm}^{-1}$ ): $v=2936,2040,2025,1769,1388,1289,1264,1134,1093$, 998, 920, 700. HRMS (ESI): $m / z 259.1329$ calc. for $\mathrm{C}_{16} \mathrm{H}_{19} \mathrm{O}_{3}{ }^{+}[\mathrm{M}+\mathrm{H}]^{+}$, found 259.1328.

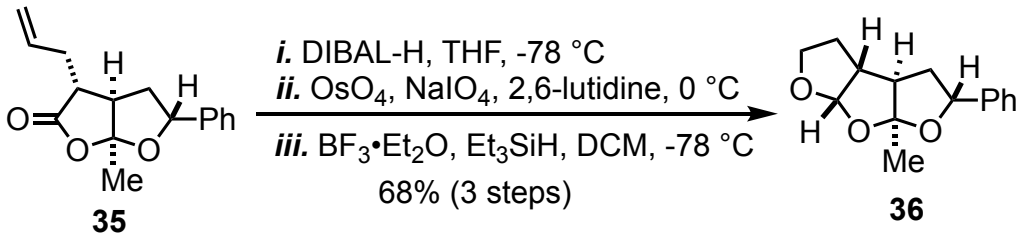


To a solution of 35 (55.3 mg, 0.214 mmol, 1.0 equiv.) in DCM (2.1 ml) was added 1.0 M DIBAL-H (1.15 equiv.) in toluene at $-78^{\circ} \mathrm{C}$. The reaction mixture was stirred for $1 \mathrm{~h}$ followed by quenching with a saturated solution of sodium potassium tartrate. After stirring another $2 \mathrm{~h}$, the organic layer was separated and the aqueous layer was further extracted with DCM. The combined organic layer was washed with brine and dried over $\mathrm{Na}_{2} \mathrm{SO}_{4}$, and evaporated. The crude was used in the next step without purification.

To a solution of the above crude product in dioxane: water mixture $(3: 1, \mathrm{v} / \mathrm{v} ; 3 \mathrm{~mL})$ at $0{ }^{\circ} \mathrm{C}$ was added 2,6lutidine ( $25 \mu \mathrm{l}, 0.214 \mathrm{mmol}, 1.0$ equiv.) and an $\mathrm{OsO}_{4}$ solution in water $(5 \mathrm{mg} / \mathrm{ml}, 0.00214 \mathrm{mmol}, 0.01$ equiv.) followed by $\mathrm{NaIO}_{4}(137 \mathrm{mg}, 0.214 \mathrm{mmol}, 3.0$ equiv.) at room temperature. After $5 \mathrm{~h}$, the reaction was completed and quenched with aqueous sodium thiosulfate solution. The reaction mixture was exhaustively extracted with EtOAc. The combined organic layer was dried over $\mathrm{Na}_{2} \mathrm{SO}_{4}$, filtered through a celite-pad, and evaporated under vacuum to yield a crude diastereomeric tricyclic hemiacetal, which was subjected to next reaction without purification.

To a solution of the above crude $(21 \mathrm{mg}, 1.0$ equiv. $)$ in anhydrous DCM $(0.8 \mathrm{ml})$ at $-78{ }^{\circ} \mathrm{C}$, triethylsilane (39 $\mu$ l, $0.24 \mathrm{mmol}, 3.0$ equiv.) and $\mathrm{BF}_{3}$ etherate (20 $\mu 1,0.16 \mathrm{mmol}, 2.0$ equiv.) was added slowly. After 2 $\mathrm{h}$, a saturated solution of sodium bicarbonate was added to it followed by extraction with DCM. The combined organic layer was washed with brine and dried over $\mathrm{Na}_{2} \mathrm{SO}_{4}$. The solvent was removed by rotatory evaporation. The residue was purified via flash column (Hex:EA = 4:1) to give $\mathbf{3 6}(13.4 \mathrm{mg}, 68 \%$, 3 steps) as colorless oil.

36: ${ }^{1} \mathbf{H}$ NMR $\left(500 \mathrm{MHz}, \mathrm{CDCl}_{3}\right) \delta 7.36$ - $7.32(\mathrm{~m}, 5 \mathrm{H}), 5.97$ (d, $\left.J=5.1 \mathrm{~Hz}, 1 \mathrm{H}\right), 5.00(\mathrm{dd}, J=11.2,4.8 \mathrm{~Hz}$, $1 \mathrm{H}), 3.99(\mathrm{td}, J=8.4,2.5 \mathrm{~Hz}, 1 \mathrm{H}), 3.92(\mathrm{ddd}, J=10.4,8.8,5.9 \mathrm{~Hz}, 1 \mathrm{H}), 2.78(\mathrm{ddt}, J=8.8,5.3,2.7 \mathrm{~Hz}, 1 \mathrm{H})$, $2.53(\mathrm{dd}, J=8.1,3.4 \mathrm{~Hz}, 1 \mathrm{H}), 2.19-2.13(\mathrm{~m}, 2 \mathrm{H}), 2.07(\mathrm{ddd}, J=12.6,11.1,8.3 \mathrm{~Hz}, 2 \mathrm{H}), 1.71(\mathrm{~s}, 3 \mathrm{H}) .{ }^{13} \mathrm{C}$ NMR $\left(125 \mathrm{MHz}, \mathrm{CDCl}_{3}\right) \delta 140.5,128.4,127.8,126.0,117.0,109.4,79.5,66.7,53.9,50.5,43.0,33.2,25.3$. IR (neat, $\left.\mathrm{cm}^{-1}\right): v=2935,2871,1449,1380,1134,1026,989,945,895,759,700$. HRMS (ESI): $\mathrm{m} / \mathrm{z}$ 247.1329 calc. for $\mathrm{C}_{15} \mathrm{H}_{19} \mathrm{O}_{3}{ }^{+}[\mathrm{M}+\mathrm{H}]^{+}$, found 247.1327 .

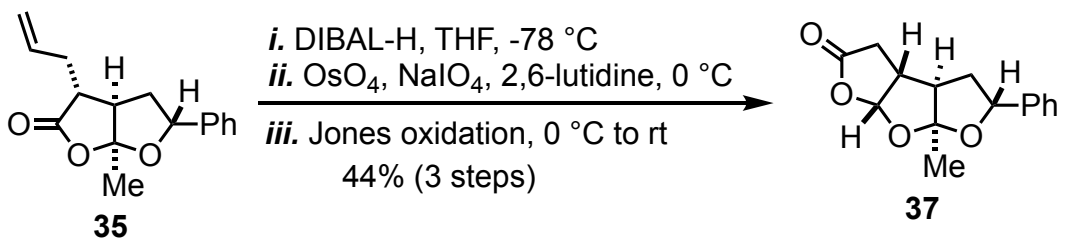

To a solution of 35 (55.3 mg, $0.214 \mathrm{mmol}, 1.0$ equiv.) in DCM (2.1 ml) was added 1.0 M DIBAL-H (1.15 equiv.) in toluene at $-78^{\circ} \mathrm{C}$. The reaction mixture was stirred for $1 \mathrm{~h}$ followed by quenching with a saturated solution of sodium potassium tartrate. After stirring another $2 \mathrm{~h}$, the organic layer was separated and the 
aqueous layer was further extracted with DCM. The combined organic layer was washed with brine and dried over $\mathrm{Na}_{2} \mathrm{SO}_{4}$ and evaporated. The crude was used to the next step without purification.

To a solution of the above crude product in dioxane/water mixture $(3: 1, \mathrm{v} / \mathrm{v} ; 3 \mathrm{~mL})$ at $0{ }^{\circ} \mathrm{C}$ was added 2,6lutidine ( $25 \mu \mathrm{l}, 0.214 \mathrm{mmol}, 1.0$ equiv.) and $5 \mathrm{mg} / \mathrm{ml} \mathrm{OsO}_{4}$ solution in water ( $0.00214 \mathrm{mmol}, 0.01$ equiv.) followed by $\mathrm{NaIO}_{4}(137 \mathrm{mg}, 0.214 \mathrm{mmol}, 3.0$ equiv.) at room temperature. After $5 \mathrm{~h}$, the reaction was quenched with an aqueous sodium thiosulfate solution and exhaustively extracted with EtOAc. The combined organic layer was dried over $\mathrm{Na}_{2} \mathrm{SO}_{4}$, filtered through a celite-pad, and evaporated under vacuum to yield a crude diastereomeric tricyclic hemiacetal, which was subjected to next reaction without purification.

To a solution of the above crude product $(23.0 \mathrm{mg}, 0.088 \mathrm{mmol}, 1.0$ equiv.) in acetone $(1 \mathrm{~mL})$ was added dropwise $2.5 \mathrm{M}$ Jones reagent ( $35 \mu 1,0.088 \mathrm{mmol}, 1.0$ equiv.) at $0{ }^{\circ} \mathrm{C}$ and the reaction mixture was gradually allowed to warm to room temperature. After $10 \mathrm{~h}$, the reaction was quenched with a sat. $\mathrm{NaHCO}_{3}$ solution and extracted with EtOAc. The combined organic layer was washed with brine and dried over $\mathrm{Na}_{2} \mathrm{SO}_{4}$, evaporated and subjected to column chromatography (Hex:EA $=3: 1$ to pure EtOAc) to yield tricyclic lactone 37 (9.9 mg, 44\%, 3 steps) as colorless oil.

37: ${ }^{1} \mathbf{H}$ NMR $\left(500 \mathrm{MHz}, \mathrm{CDCl}_{3}\right) \delta 7.38-7.29(\mathrm{~m}, 5 \mathrm{H}), 6.21$ (d, $\left.J=5.4 \mathrm{~Hz}, 1 \mathrm{H}\right), 5.06(\mathrm{dd}, J=9.1,6.8 \mathrm{~Hz}$, $1 \mathrm{H}), 3.05$ (tdd, $J=6.9,3.4,1.8 \mathrm{~Hz}, 1 \mathrm{H}), 2.93$ (dd, $J=18.1,9.3 \mathrm{~Hz}, 1 \mathrm{H}), 2.63$ (dd, $J=18.1,2.5 \mathrm{~Hz}, 1 \mathrm{H}$ ), $2.54(\mathrm{q}, J=4.5 \mathrm{~Hz}, 1 \mathrm{H}), 2.19-2.13(\mathrm{~m}, 2 \mathrm{H}), 1.74(\mathrm{~s}, 3 \mathrm{H}) .{ }^{13} \mathbf{C}$ NMR $\left(125 \mathrm{MHz}, \mathrm{CDCl}_{3}\right) \delta{ }^{13} \mathrm{C}$ NMR $(125$ $\left.\mathrm{MHz}, \mathrm{CDCl}_{3}\right) \delta 174.0,139.3,128.6,128.1,126.0,119.6,107.4,79.9,53.6,46.6,42.2,36.3,26.1$. IR (neat, $\left.\mathrm{cm}^{-1}\right): v=2918,2026,2010,1782,1174,1102,1005,944,886,701$. HRMS (ESI): $\mathrm{m} / z 261.1121$ calc. for $\mathrm{C}_{15} \mathrm{H}_{17} \mathrm{O}_{4}^{+}[\mathrm{M}+\mathrm{H}]^{+}$, found 261.1119 . 


\section{Part 2. X-Ray structure and analysis data.}

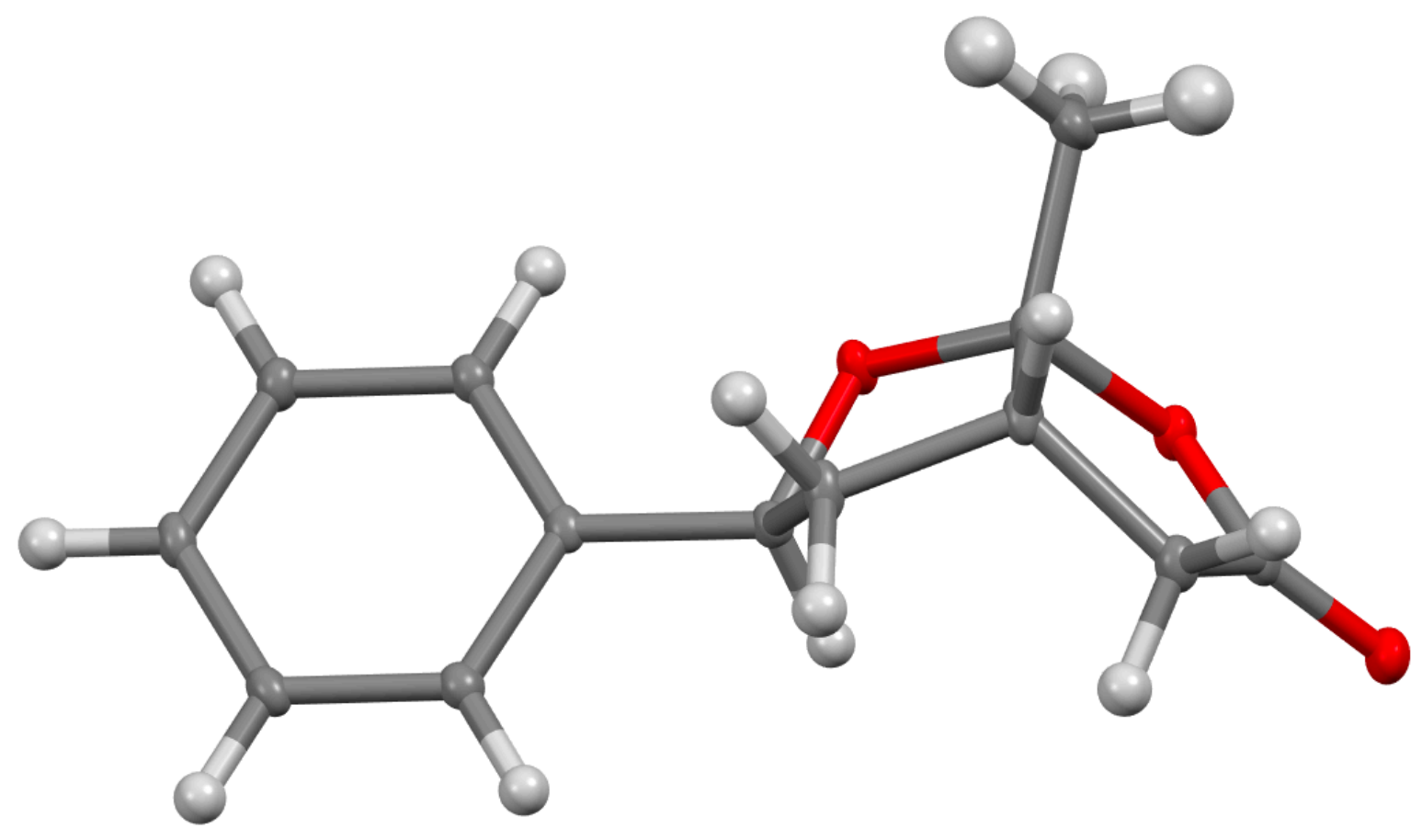

Figure S1. X-ray Structure of 16a

A colorless plate shaped crystal of $\mathbf{1 6 a}$ for X-ray diffraction was obtained by slow evaporation of a Hexanes/EtOAc solution of 16a. The data were collected at 150(2) K on a Bruker AXS D8 Quest CMOS diffractometer with Mo sealed tube and curved triumph monochromator with a $10 \mathrm{~cm}$ x $10 \mathrm{~cm}$ Photon-100 detector and fixed chi angle. The supplementary crystallographic data was deposited in The Cambridge Crystallographic Data Centre. CCDC1997763.

\section{X-ray analysis data:}

Bond precision: $\mathrm{C}-\mathrm{C}=0.0022$ A Wavelength $=1.54178$

Cell: $a=11.8558(4) b=13.1276(4) c=7.1585(2)$

alpha $=90$ beta $=101.6907(10)$ gamma $=90$

Temperature: 150 K Calculated Reported

Volume 1091.02(6) 1091.02(6)

Space group P 21/c P 21/c

Hall group -P 2ybc-P 2ybc

Moiety formula C13 H14 O3 ?

Sum formula C13 H14 O3 C13 H14 O3

Mr 218.24 218.24 
Dx,g cm-3 1.3291 .329

Z 44

$\mathrm{Mu}(\mathrm{mm}-1) 0.7660 .766$

F000 464.0464 .0

F000' 465.48

h,k,lmax 14,16,8 14,16,8

Nref 21542113

Tmin,Tmax 0.752,0.948 0.510,0.754

Tmin' 0.708

Correction method $=\#$ Reported T Limits: Tmin $=0.510$

Tmax $=0.754$ AbsCorr $=$ MULTI-SCAN

Data completeness $=0.981$ Theta $(\max )=72.204$

$\mathrm{R}($ reflections $)=0.0516(1968) \mathrm{wR} 2$ (reflections $)=0.1600(2113)$

$\mathrm{S}=1.107$ Npar $=147$ 


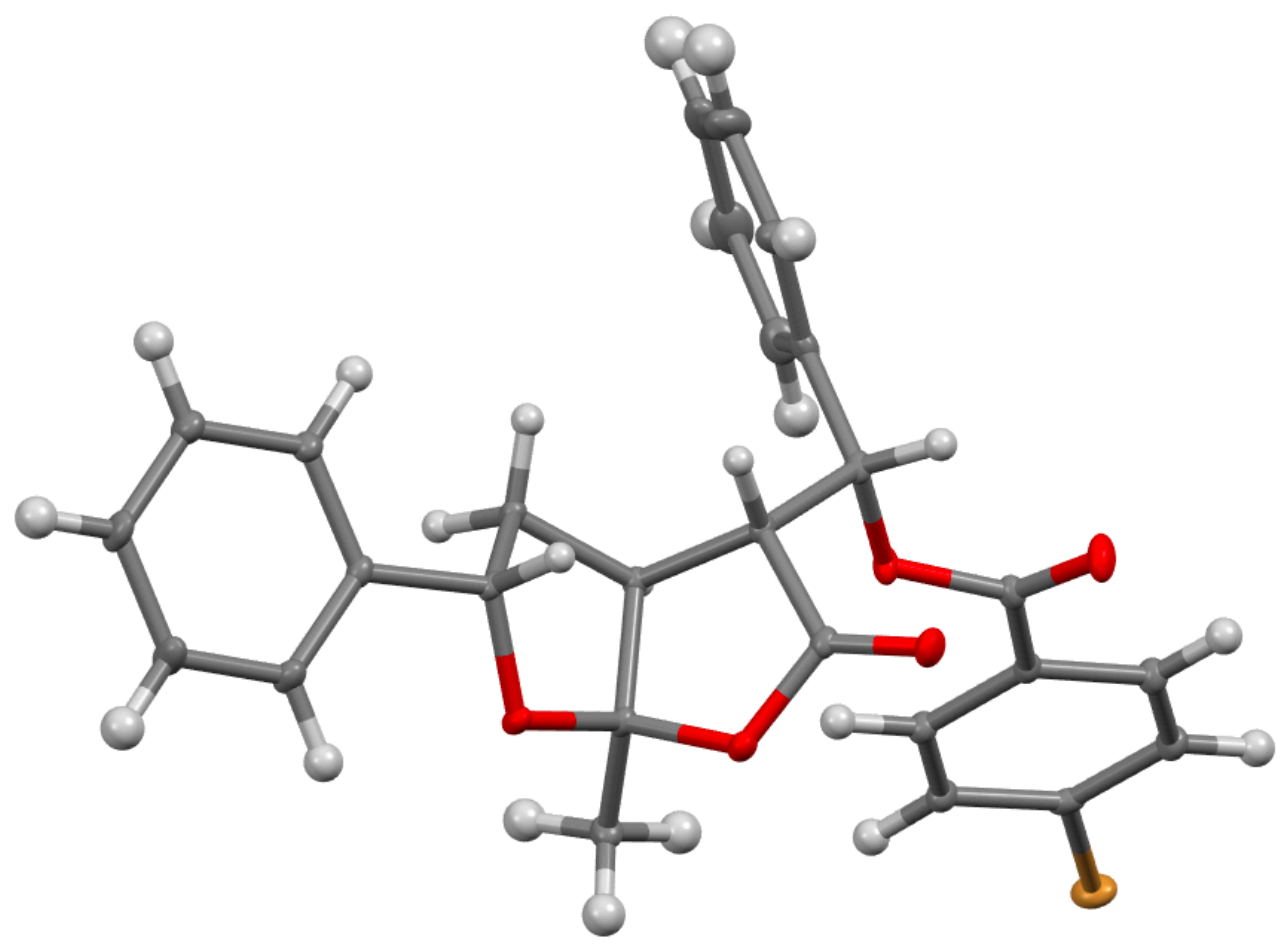

Figure S2. X-ray Structure of $\mathbf{3 2}$

A colorless plate shaped crystal of $\mathbf{3 2}$ for X-ray diffraction was obtained by slow evaporation of a isopropyl alcohol/EtOAc solution of 32. The data were collected at 150(2) K on a Bruker AXS D8 Quest CMOS diffractometer with Mo sealed tube and curved triumph monochromator with a $10 \mathrm{~cm}$ x $10 \mathrm{~cm}$ Photon-100 detector and fixed chi angle. The supplementary crystallographic data was deposited in The Cambridge Crystallographic Data Centre. CCDC 2016932.

Bond precision: $\mathrm{C}-\mathrm{C}=0.0048$ A Wavelength $=1.54178$

Cell: $\mathrm{a}=10.1739(3) \mathrm{b}=10.3086(3) \mathrm{c}=11.0993(3)$

alpha $=90$ beta $=95.0877(14)$ gamma $=90$

Temperature: $150 \mathrm{~K}$

Calculated Reported

Volume $\quad 1159.49(6)$ 1159.49(11)

Space group P $21 \quad$ P 21

Hall group $\quad \mathrm{P} 2 \mathrm{yb} \quad \mathrm{P} 2 \mathrm{yb}$

Moiety formula $\mathrm{C} 27 \mathrm{H} 23 \mathrm{Br} \mathrm{O} 5$ ?

Sum formula $\mathrm{C} 27 \mathrm{H} 23 \mathrm{Br}$ O5 $\mathrm{C} 27 \mathrm{H} 23 \mathrm{Br} \mathrm{O} 5$

$\mathrm{Mr} \quad 507.35 \quad 507.36$

Dx,g cm-3 $\quad 1.453 \quad 1.453$

$\begin{array}{lll}\mathrm{Z} & 2 & 2\end{array}$ 


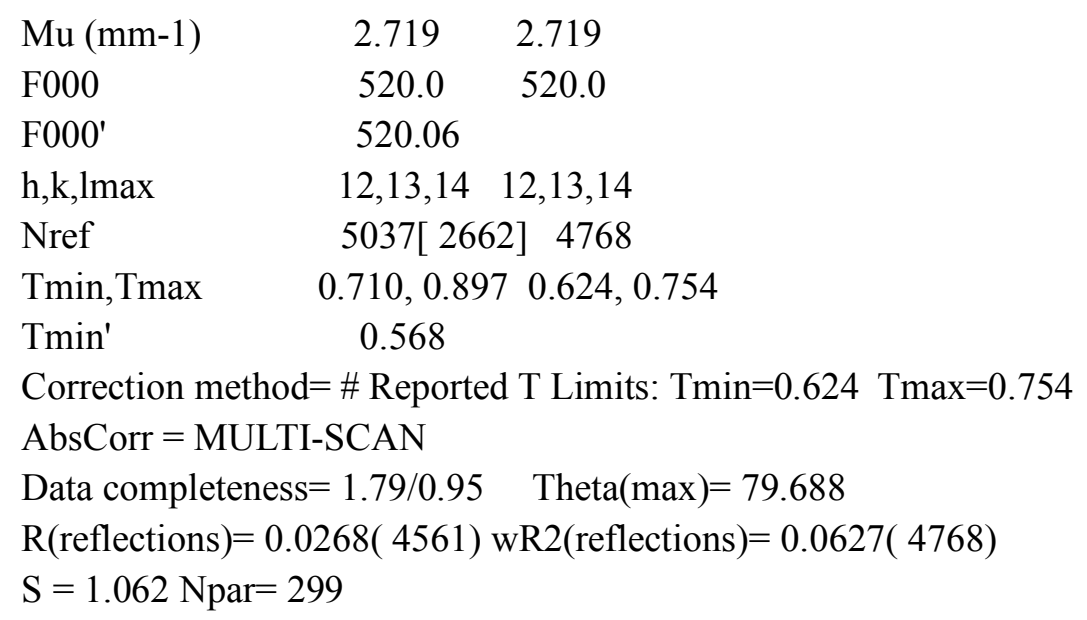

\section{Reference:}

1. Quan, L. G.; Kim, S.-H.; Lee, J. C.; Cha, J. K. Diastereoselective Synthesis of trans-1,2Dialkylcyclopropanols by the Kulinkovich Hydroxycyclopropanation of Homoallylic Alcohols. Angew. Chem. Int. Ed. 2002, 41, 2160-2162.

2. Sun, H.; Roush, W. S.; Hughes, D. Synthesis of (+)-B-Allyldiisopinocampheylborane and its Reaction with Aldehydes. Org. Synth. 2011, 88, 87-101.

3. Lohse-Fraefel, N.; Carreira, E. M. Polyketide Building Blocks via Diastereoselective Nitrile Oxide Cycloadditions with Homoallylic Alcohols and Monoprotected Homoallylic Diols. Chem. Eur. J. 2009, 15, 12065-12081.

4. Ueda, Y.; Abe, H.; Iguchi, K.; Ito, H. Synthetic Study of Yonarolide: Stereoselective Construction of the Tricyclic Core. Tetrahedron Lett. 2011, 52, 3379-3381. 
Part 3. ${ }^{1} \mathrm{H},{ }^{13} \mathrm{C}$, and ${ }^{19} \mathrm{~F}$ NMR spectra

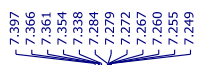<smiles>C[C@@]1(O)C[C@@H]1CC(O)c1ccccc1</smiles>

${ }^{1} \mathrm{H}$ NMR (500 MHz, $\mathrm{CDCl}_{3}$ )
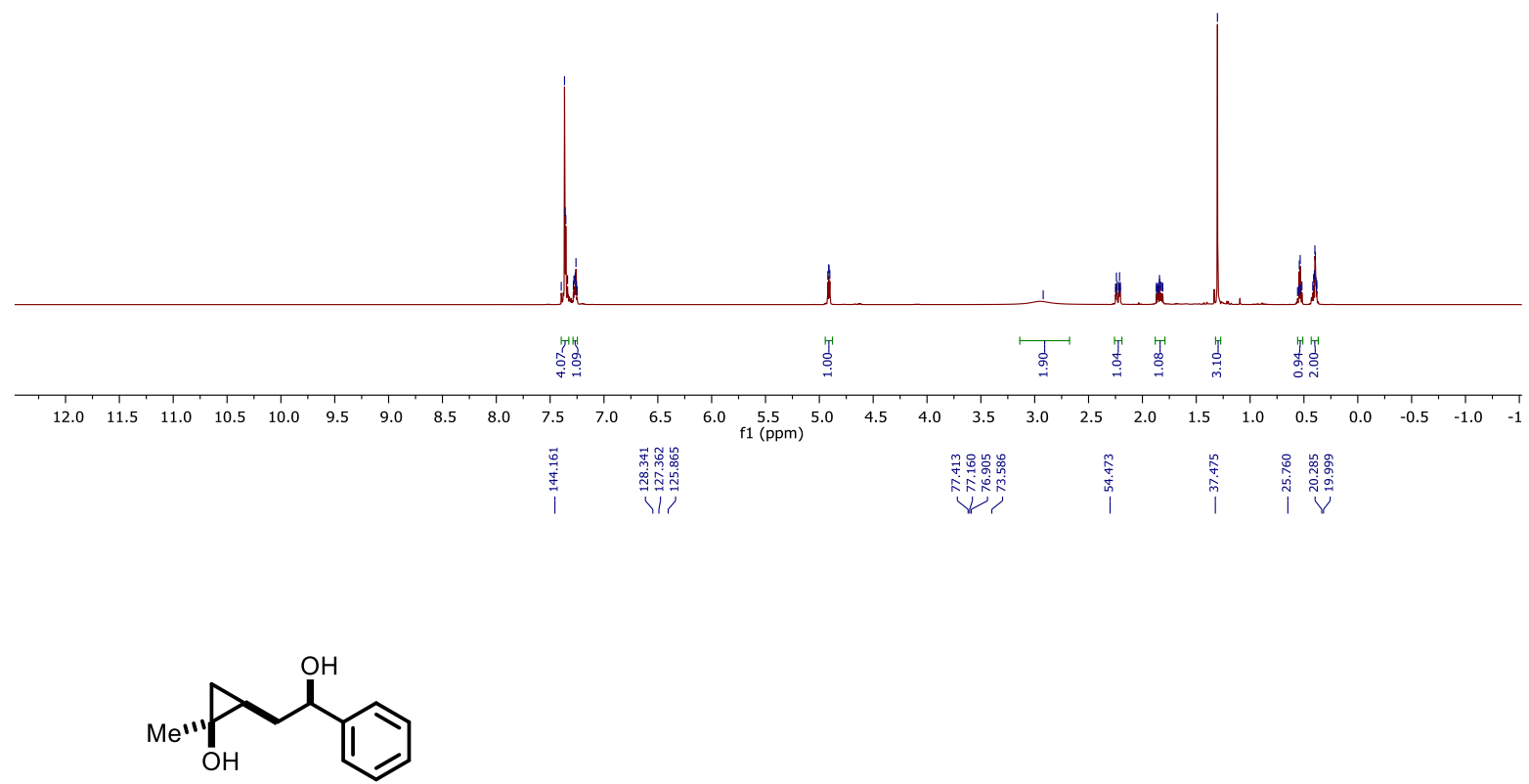

${ }^{13} \mathrm{C}$ NMR $\left(125 \mathrm{MHz}, \mathrm{CDCl}_{3}\right)$

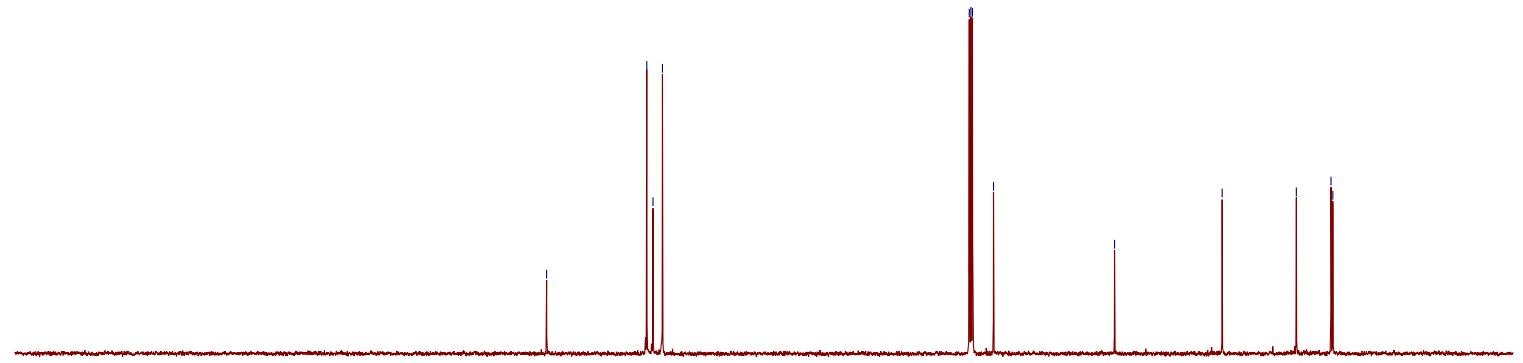

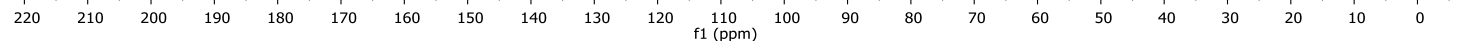




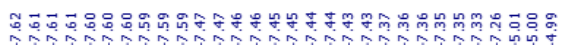

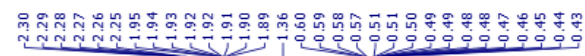

(N)

${ }^{1} \mathrm{H}$ NMR (500 MHz, $\mathrm{CDCl}_{3}$ )
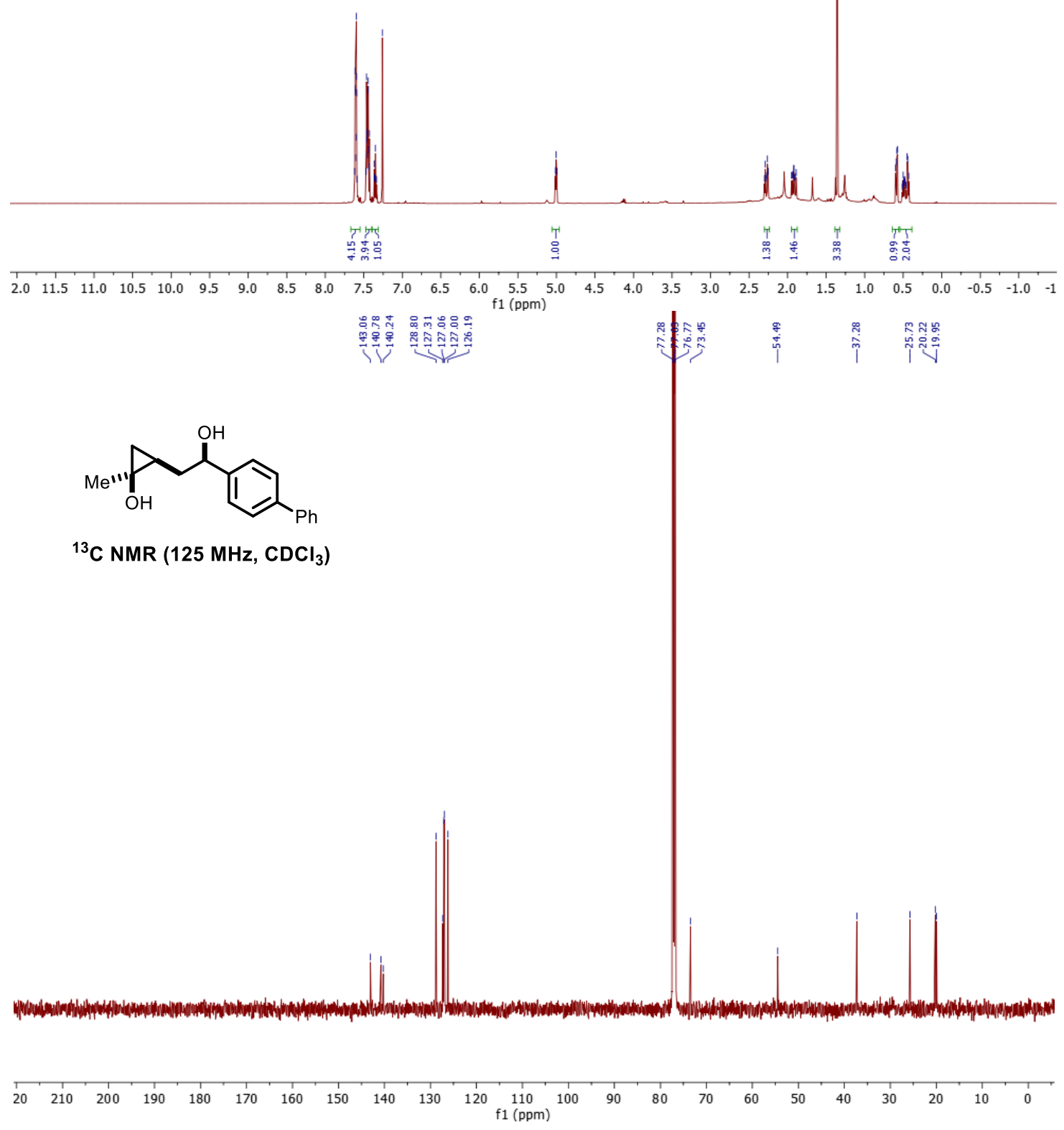

S38 
<smiles>C[C@]1(O)C[C@@H]1CC(O)c1ccc([N+](=O)[O-])cc1</smiles>

${ }^{1} \mathrm{H}$ NMR $\left(500 \mathrm{MHz}, \mathrm{CDCl}_{3}\right)$

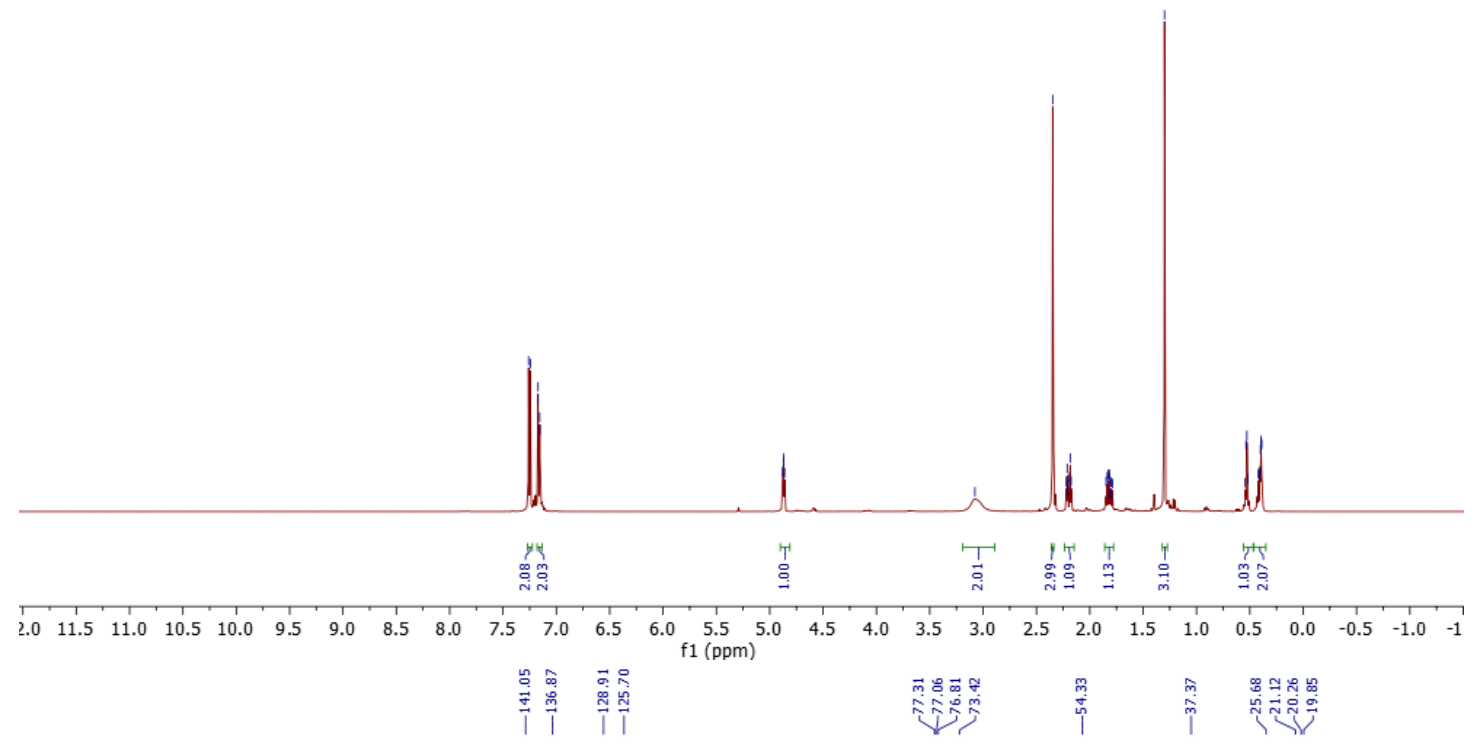<smiles>C[C@]1(O)C[C@@H]1CC(O)c1ccc([N+](=O)[O-])cc1</smiles>

${ }^{13} \mathrm{C}$ NMR (125 MHz, $\left.\mathrm{CDCl}_{3}\right)$

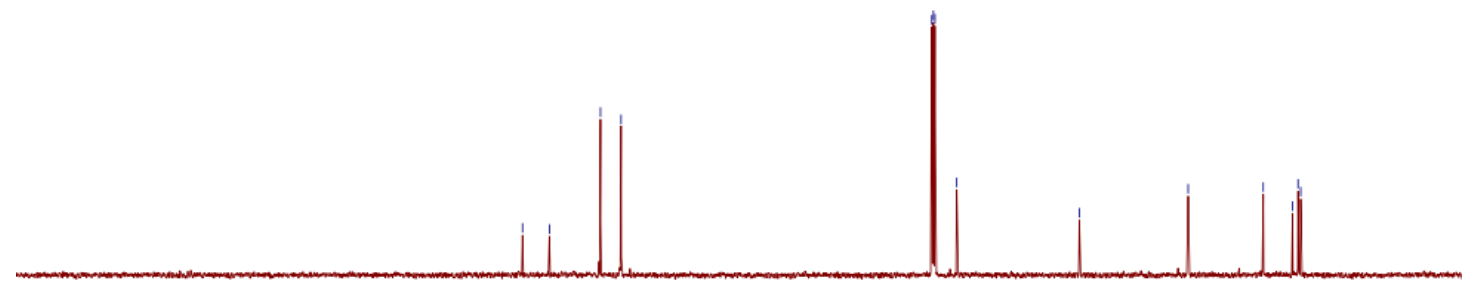

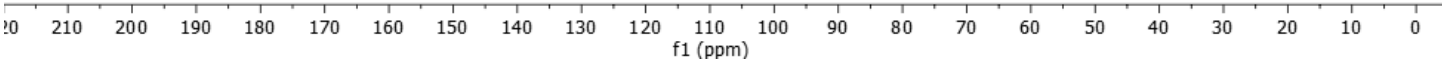




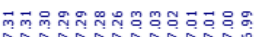

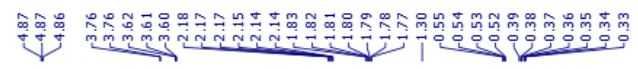

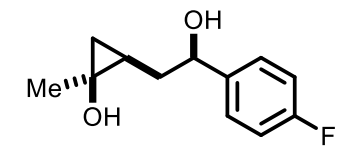

${ }^{1} \mathrm{H}$ NMR (500 MHz, $\mathrm{CDCl}_{3}$ )
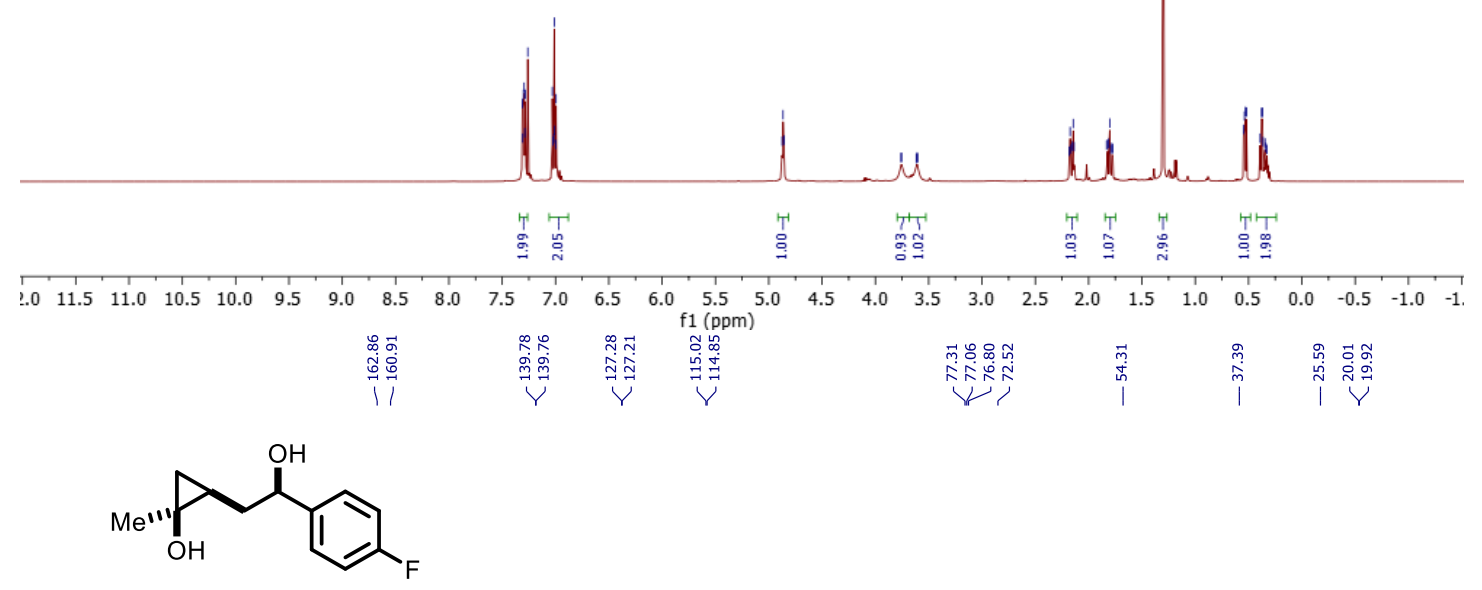

${ }^{13} \mathrm{C}$ NMR (125 MHz, $\mathrm{CDCl}_{3}$ )

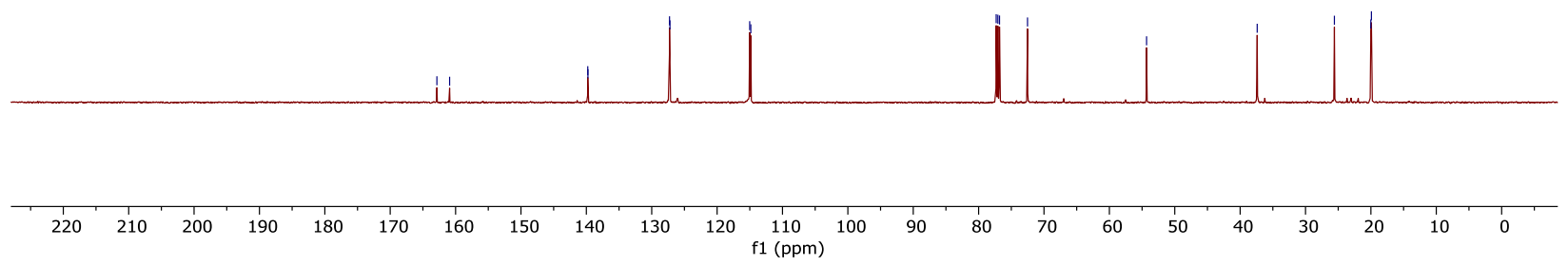

S40 


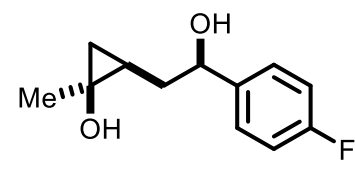

${ }^{19} \mathrm{~F}$ NMR $\left(471 \mathrm{MHz}, \mathrm{CDCl}_{3}\right)$

$\begin{array}{rllllllllllllllllllllllllllll}10 & 0 & -10 & -20 & -30 & -40 & -50 & -60 & -70 & -80 & -90 & -100 & -110 & -120 & -130 & -140 & -150 & -160 & -170 & -180 & -190 & -200 & -210 & -220\end{array}$




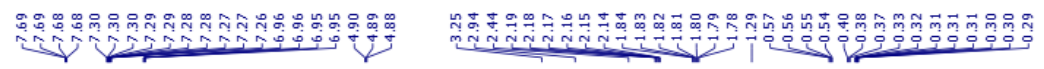

OTs

${ }^{1} \mathrm{H}$ NMR (500 MHz, $\mathrm{CDCl}_{3}$ )
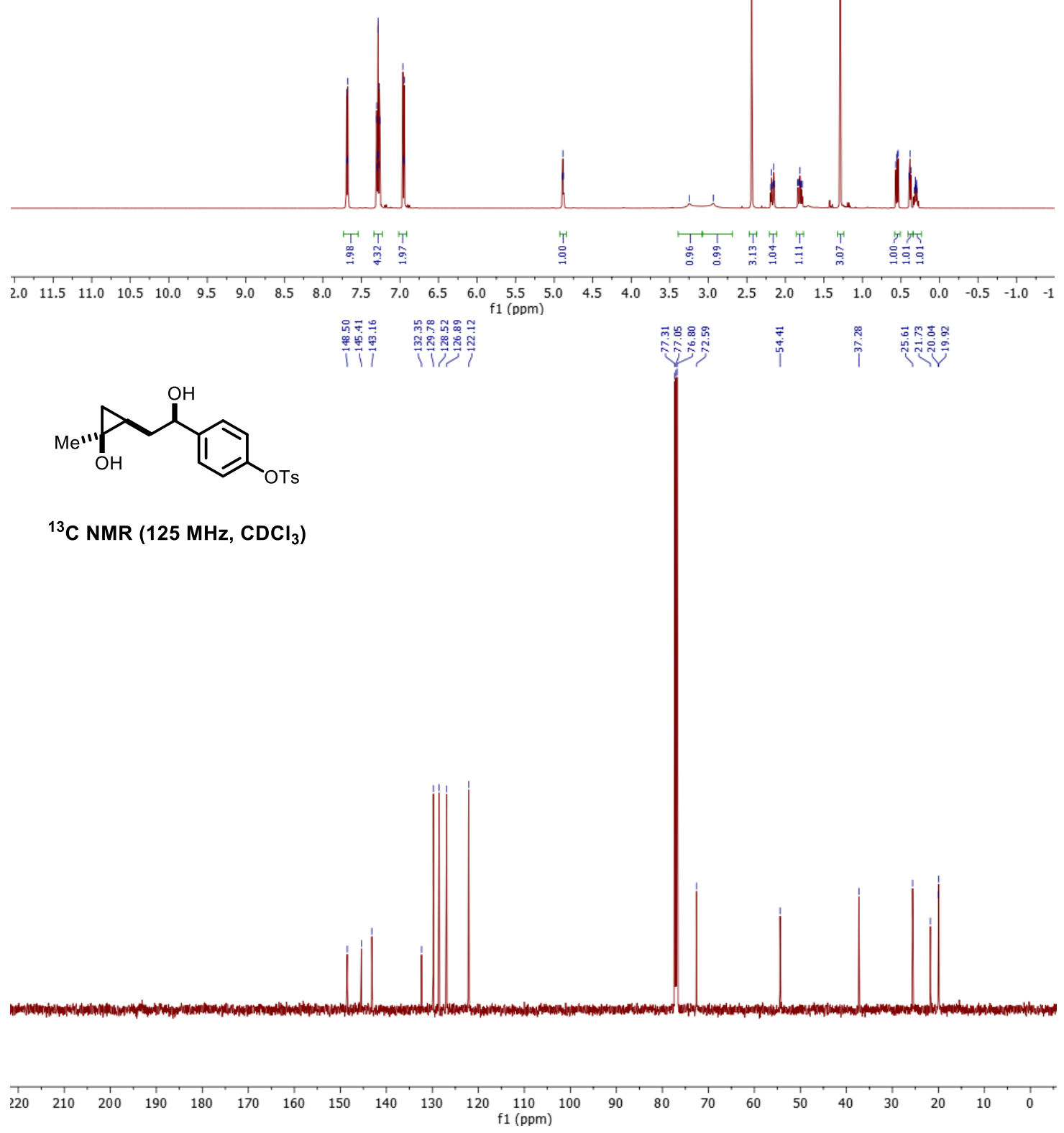

S42 


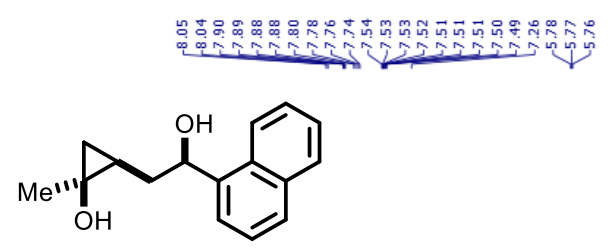

${ }^{1} \mathrm{H}$ NMR (500 MHz, $\left.\mathrm{CDCl}_{3}\right)$

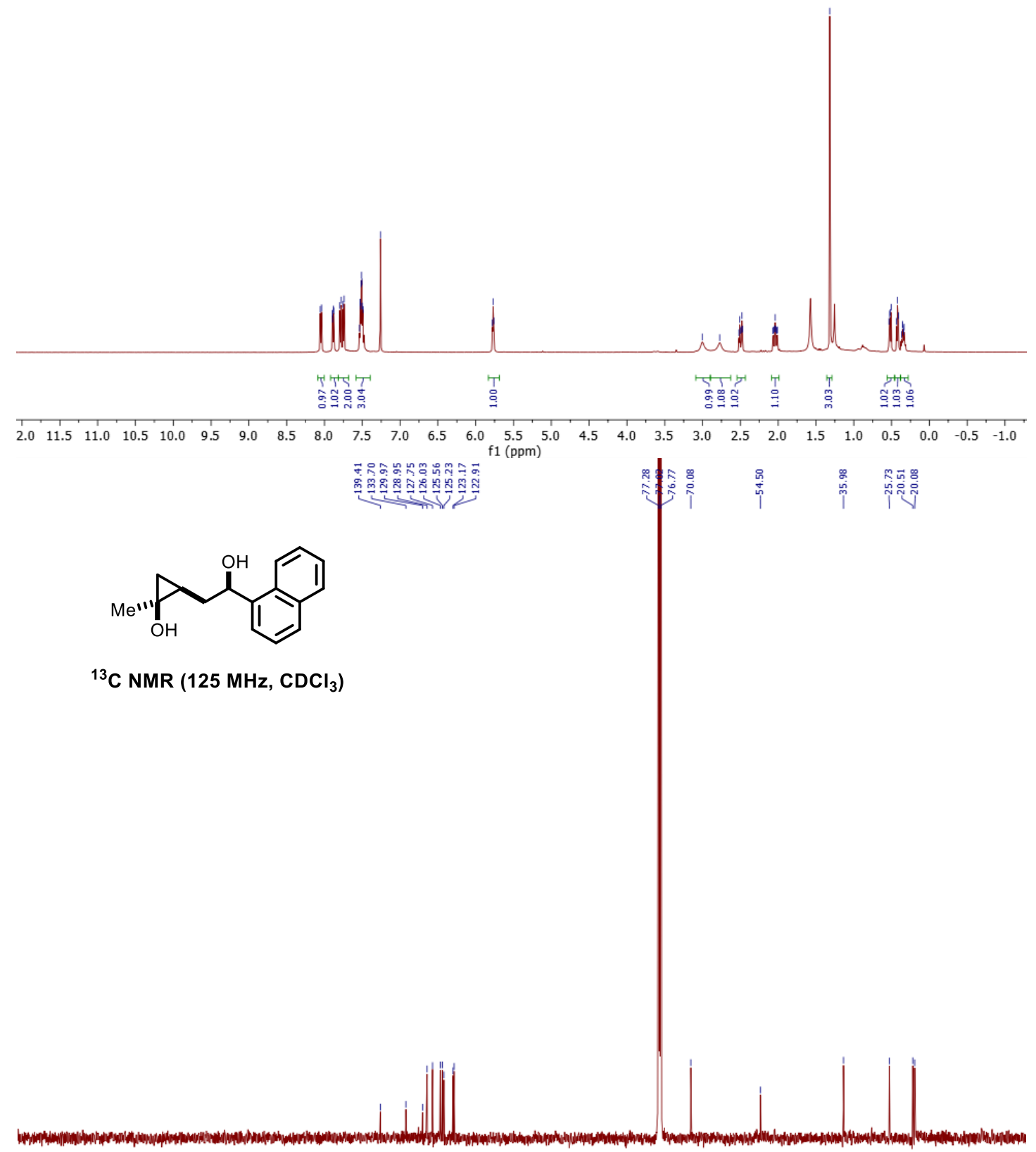

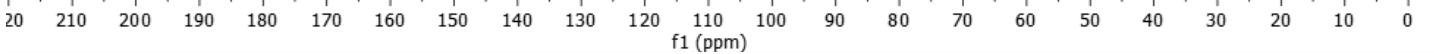




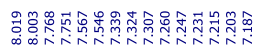

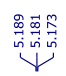

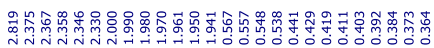

19t12

(1)

${ }^{1} \mathrm{H}$ NMR (500 MHz, $\mathrm{CDCl}_{3}$ )

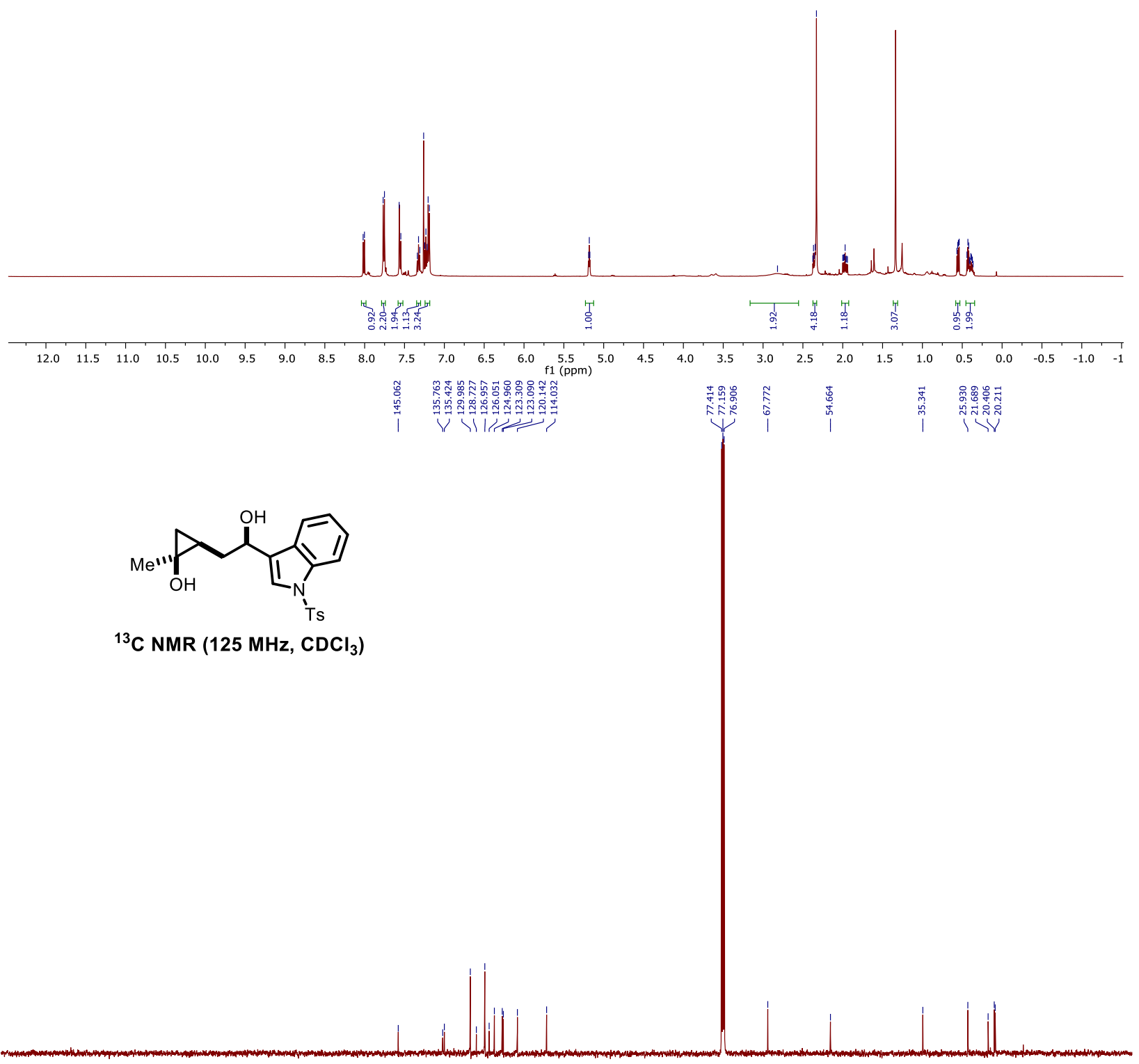

(1) 
<smiles></smiles>

${ }^{1} \mathrm{H}$ NMR (500 MHz, $\mathrm{CDCl}_{3}$ )

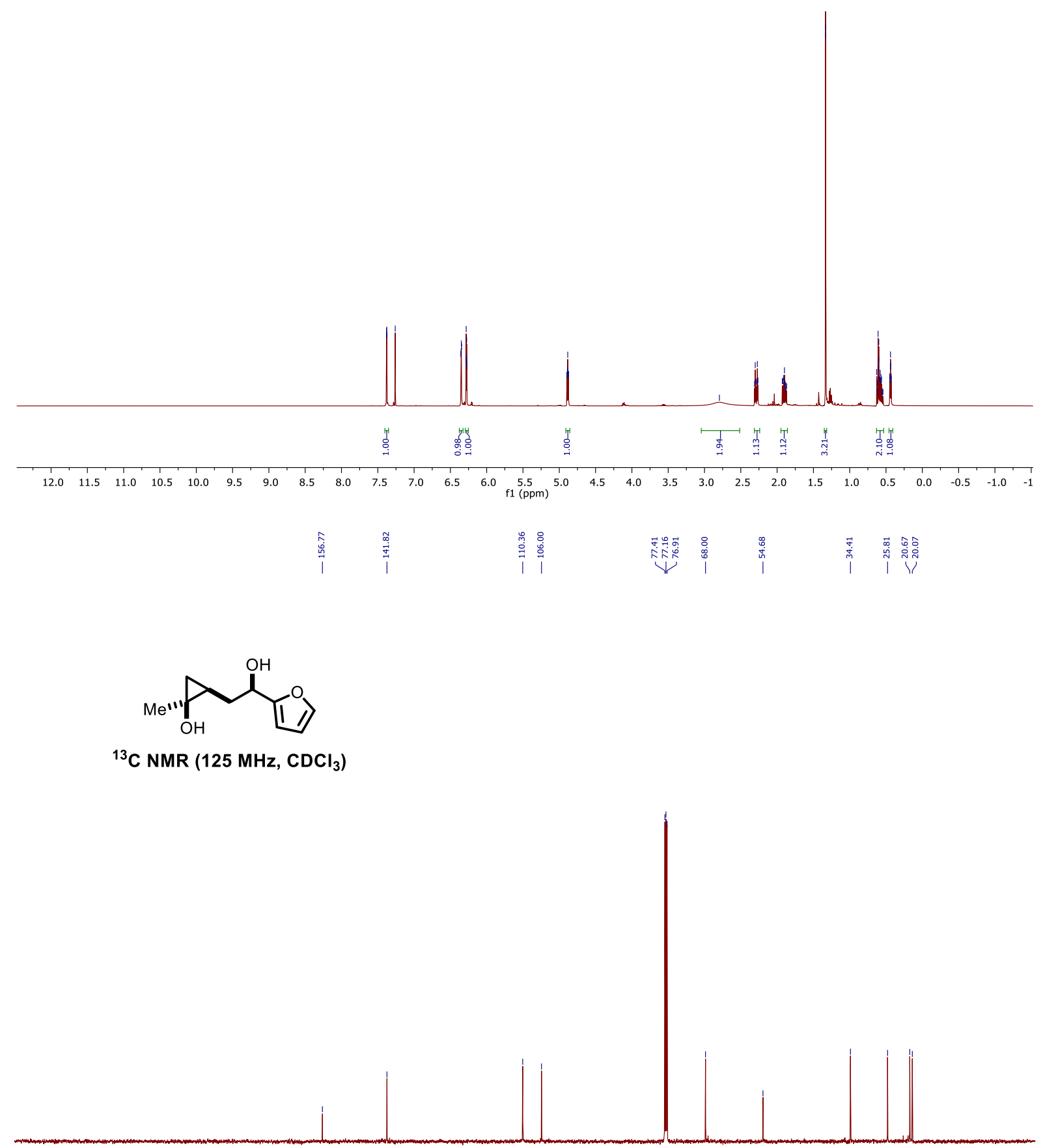



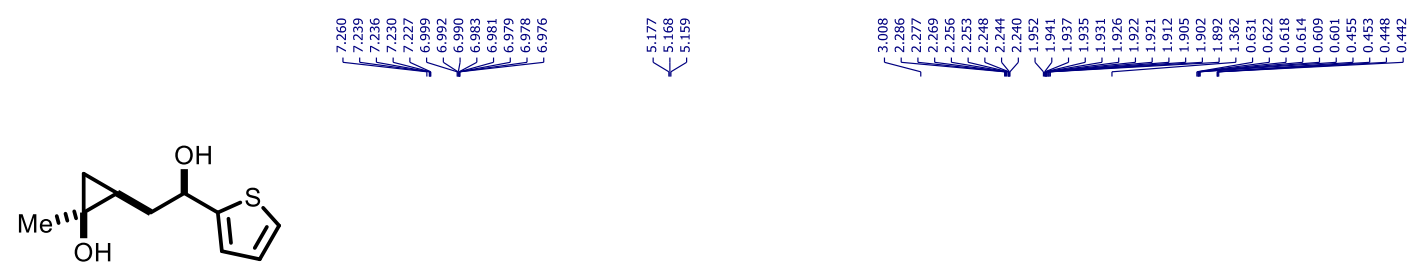

${ }^{1} \mathrm{H}$ NMR (500 MHz, $\mathrm{CDCl}_{3}$ )

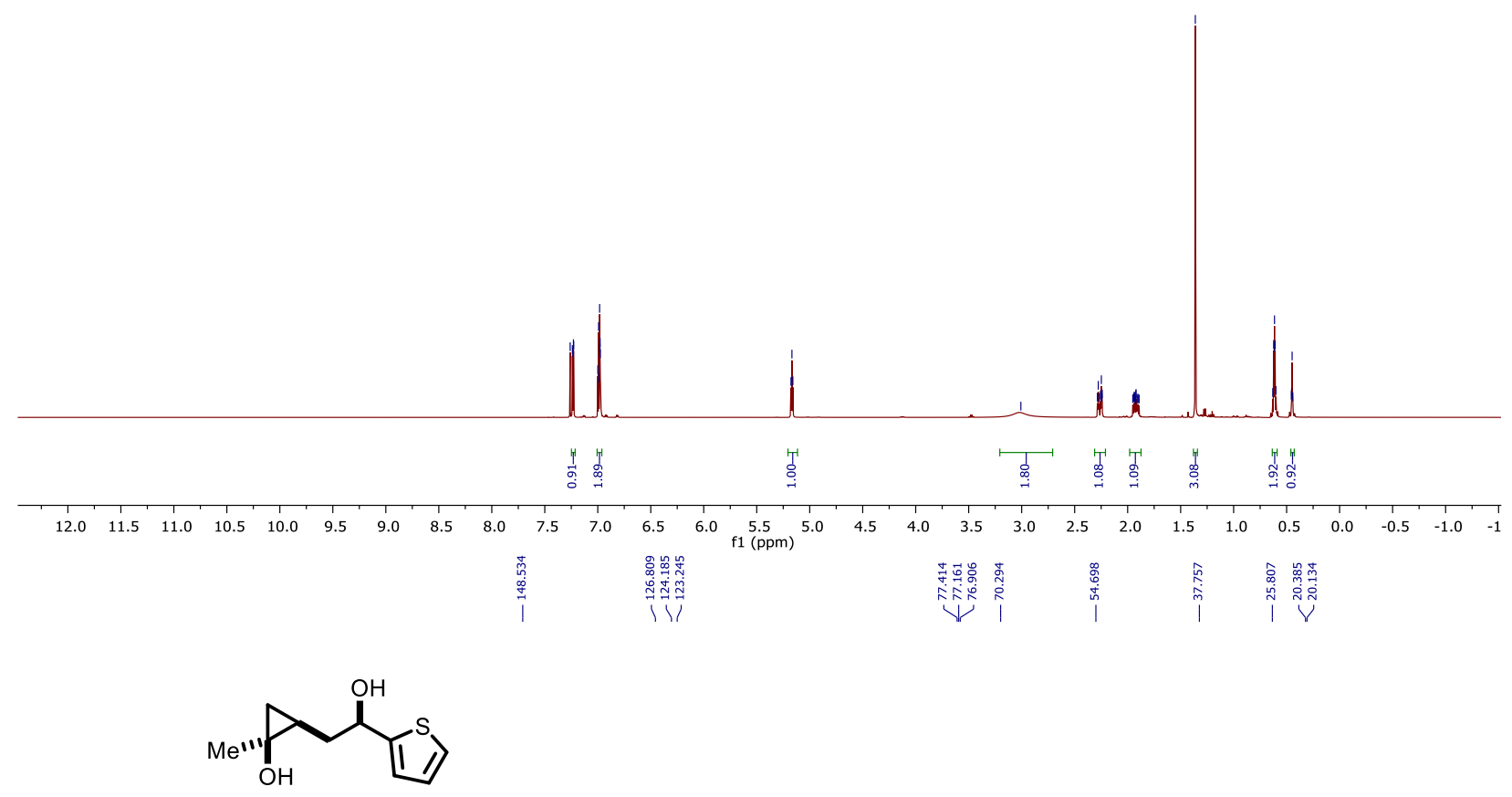

${ }^{13} \mathrm{C}$ NMR (125 MHz, $\mathrm{CDCl}_{3}$ )

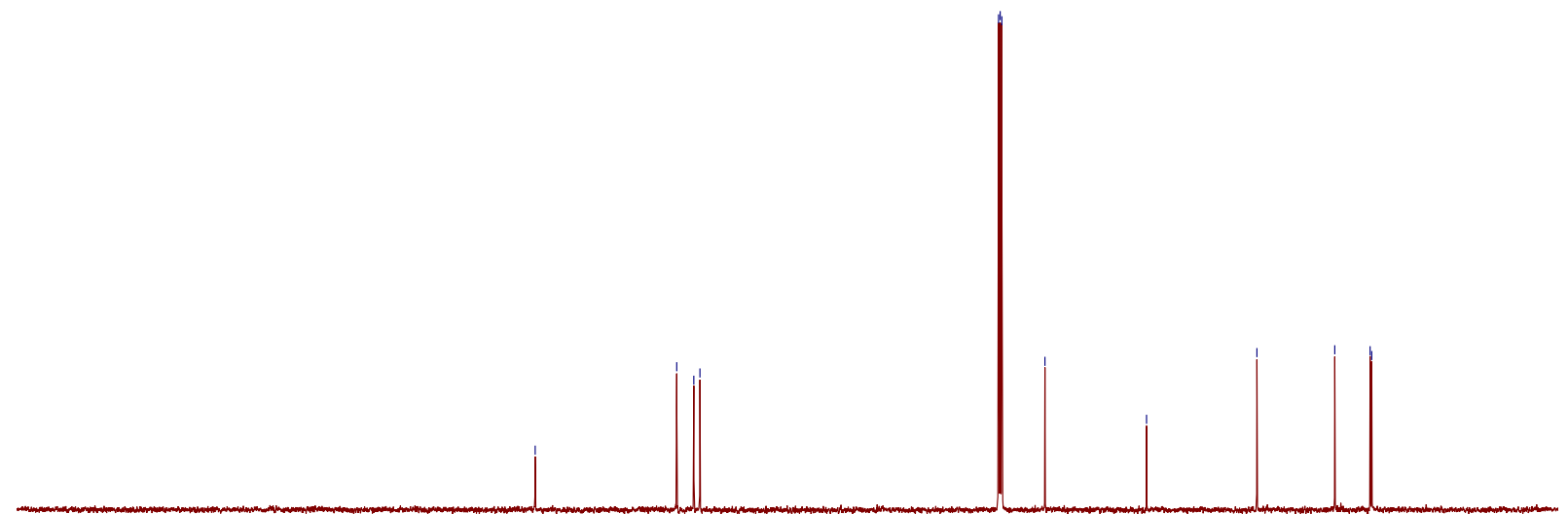

$\begin{array}{llllllllllllllllllllllll}1 & 1 \\ 220 & 210 & 200 & 190 & 180 & 170 & 160 & 150 & 140 & 130 & 120 & 110 & 100 & 90 & 80 & 70 & 60 & 50 & 40 & 30 & 20 & 10 & 0 & 10\end{array}$ 


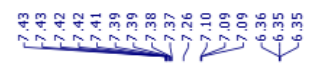

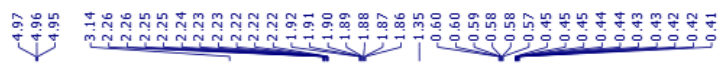

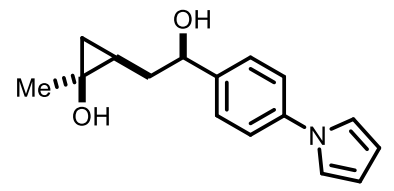

${ }^{1} \mathrm{H}$ NMR (500 MHz, $\left.\mathrm{CDCl}_{3}\right)$
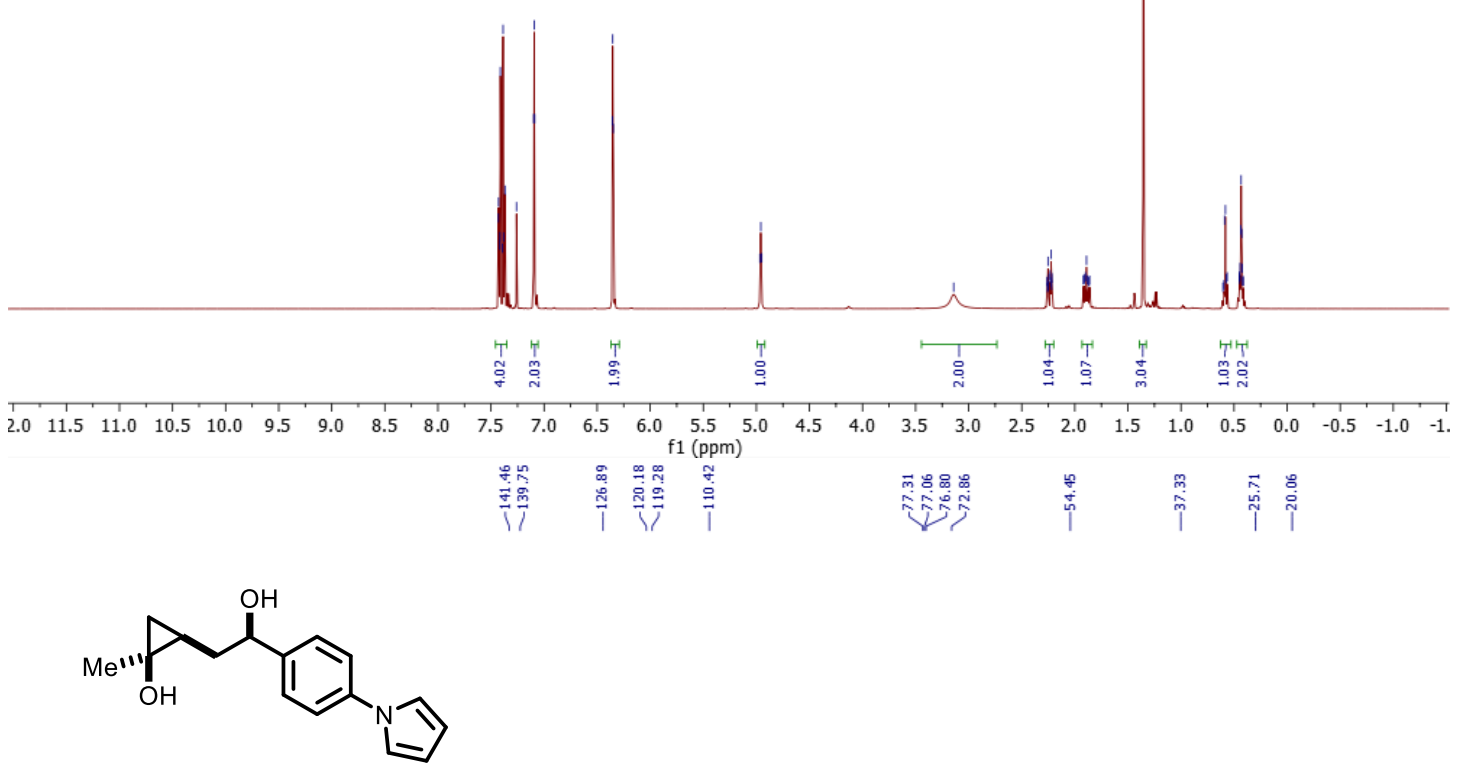

${ }^{13} \mathrm{C}$ NMR $\left(125 \mathrm{MHz}, \mathrm{CDCl}_{3}\right)$
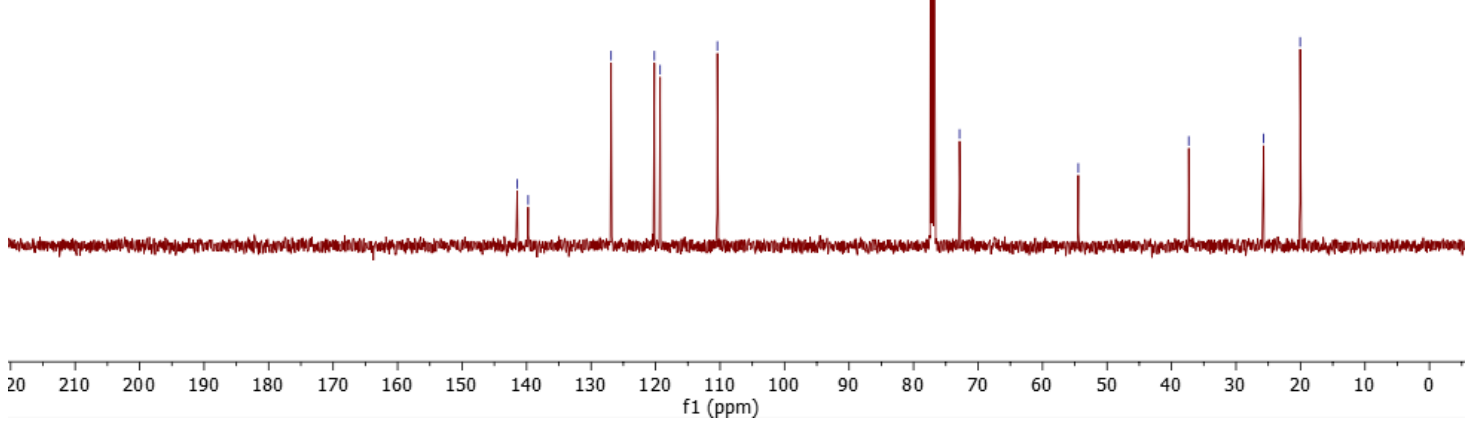

S47 
<smiles>C[C@@H]1CC1CC(O)CO[Mg]</smiles>

${ }^{1} \mathrm{H}$ NMR (500 MHz, $\left.\mathrm{CDCl}_{3}\right)$

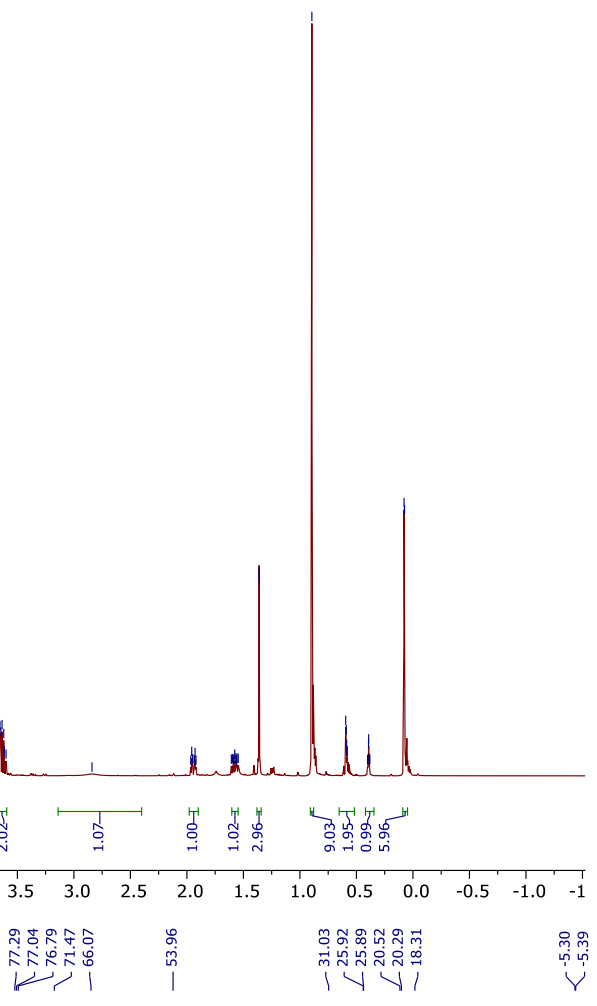<smiles>C[C@@H]1CC1CC(O)CO[Mg]</smiles>

${ }^{13} \mathrm{C} \mathrm{NMR}\left(125 \mathrm{MHz}, \mathrm{CDCl}_{3}\right)$

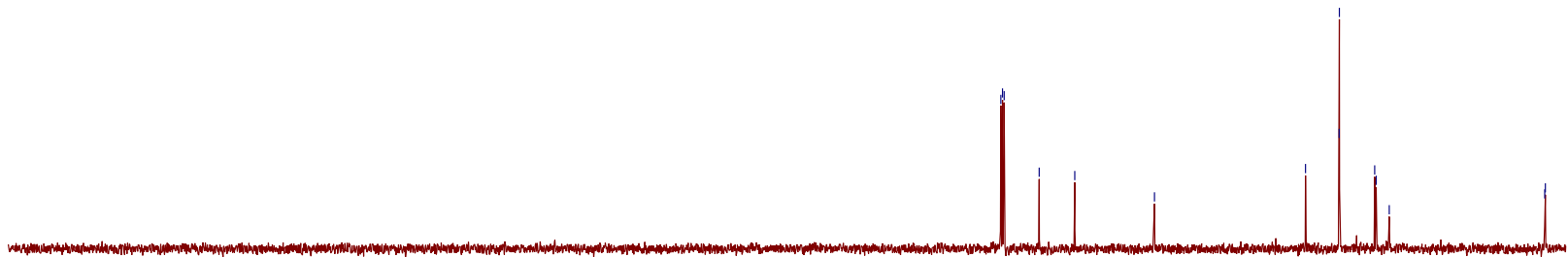




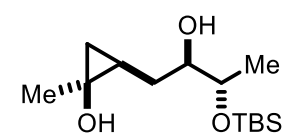

${ }^{1} \mathrm{H}$ NMR $\left(500 \mathrm{MHz}, \mathrm{CDCl}_{3}\right.$ )
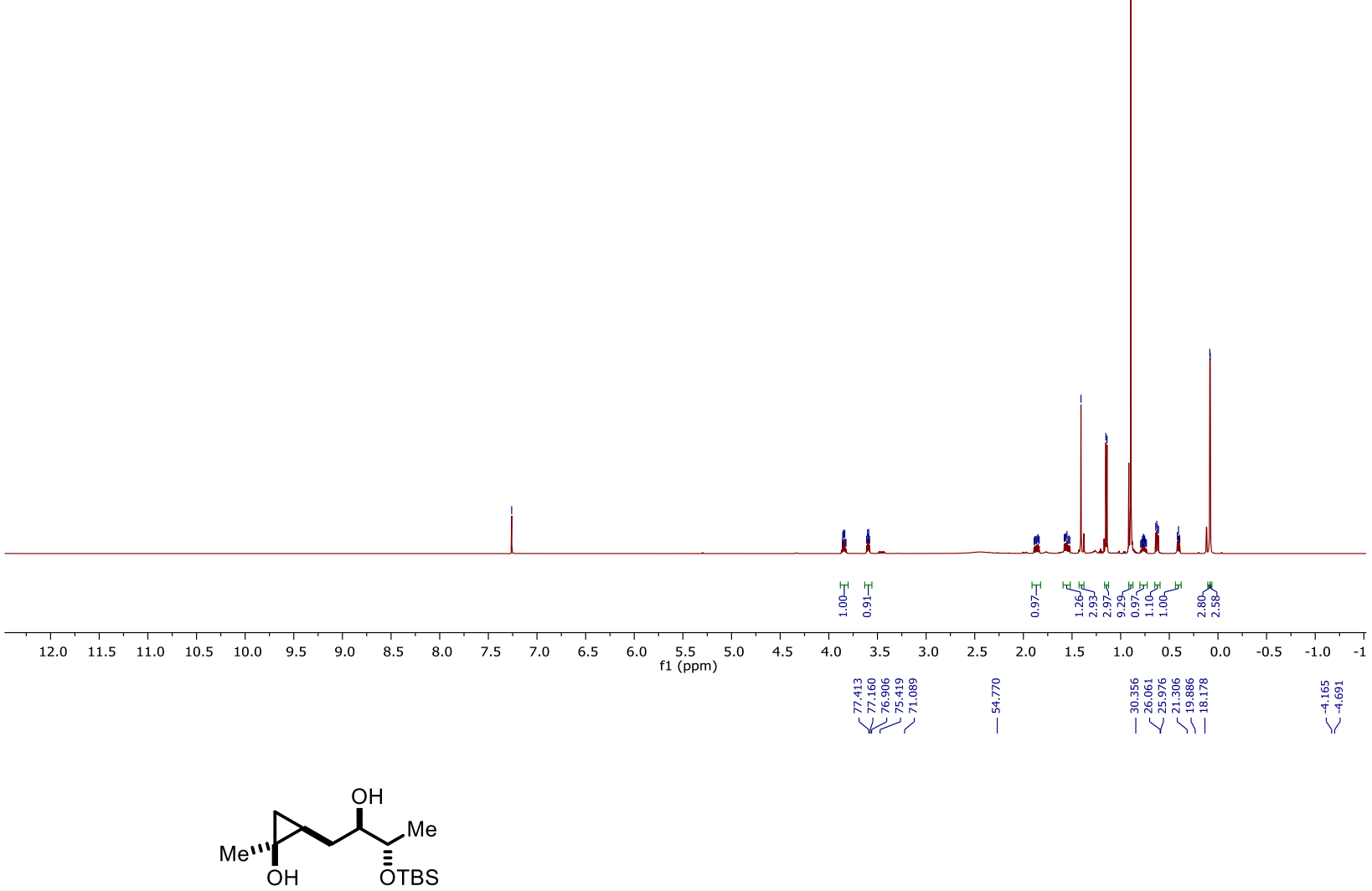

$\left.{ }^{13} \mathrm{C} \mathrm{NMR} \mathrm{(125} \mathrm{MHz,} \mathrm{CDCl}_{3}\right)$

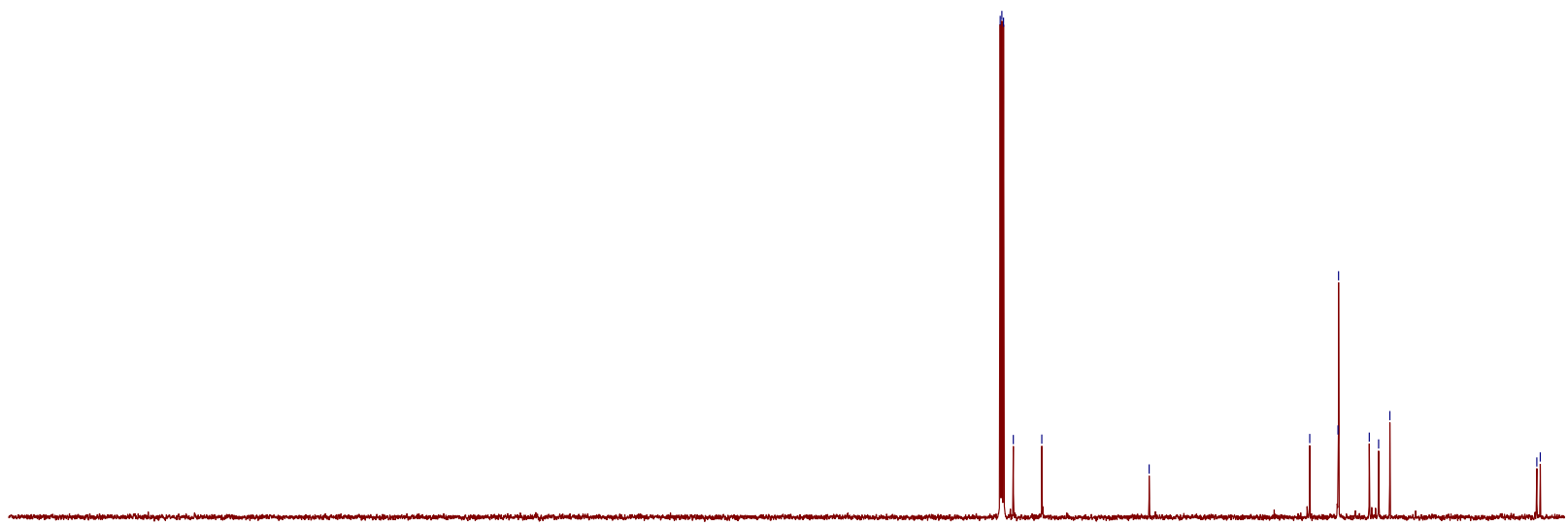

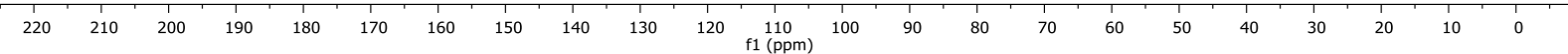


<smiles>C[C@@H]1C[C@H]1CC(O)C(C)(C)C</smiles>

${ }^{1} \mathrm{H}$ NMR (500 MHz, $\mathrm{CDCl}_{3}$ )

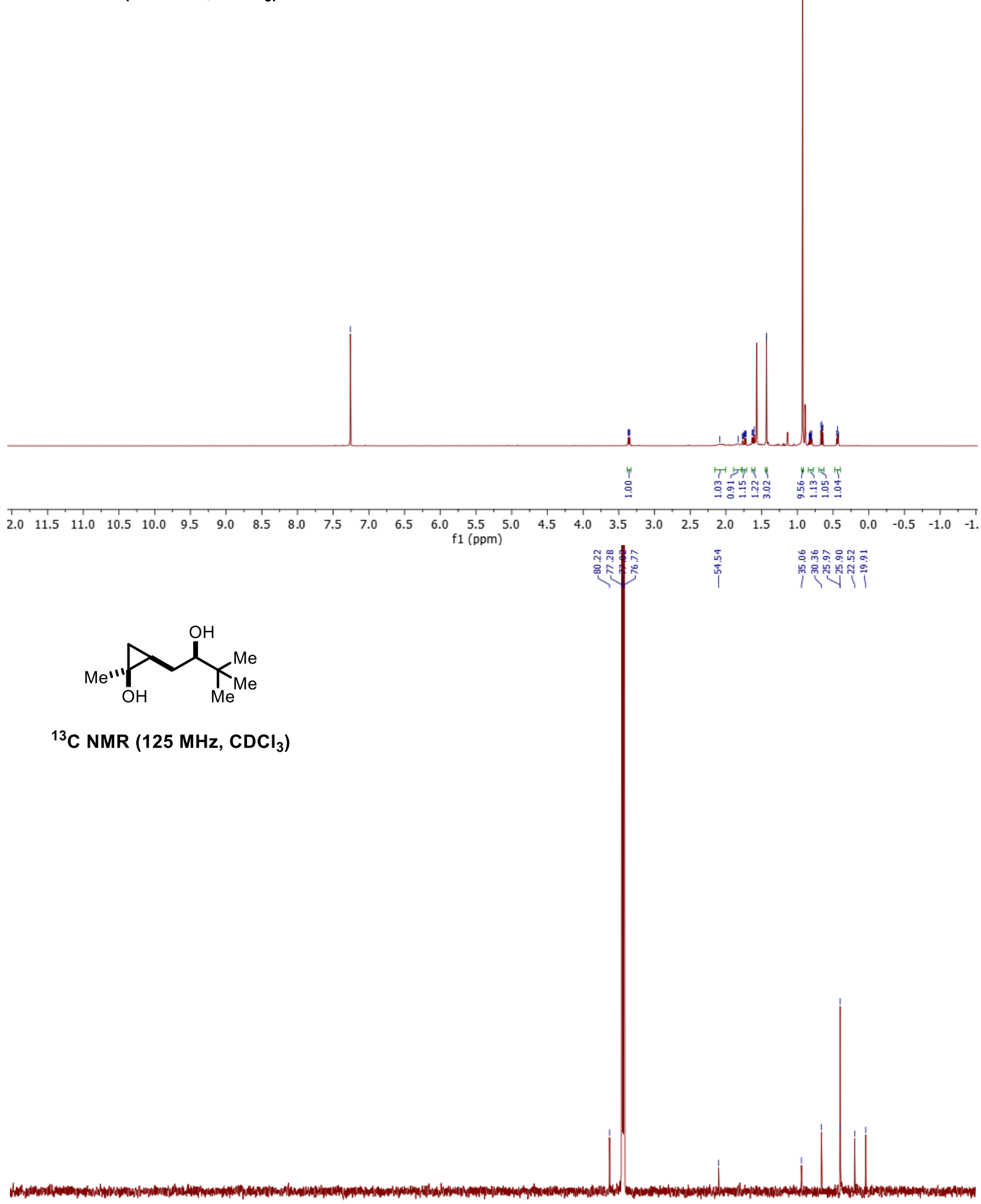

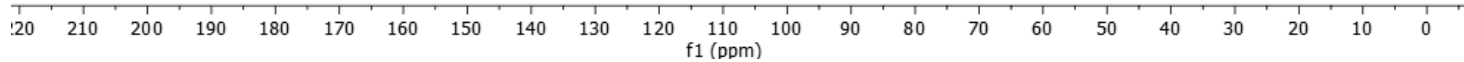


<smiles>C[C@@H]1CC1CC1(O)CCC1</smiles>

$\mathrm{OH}$

${ }^{1} \mathrm{H}$ NMR $\left(500 \mathrm{MHz}, \mathrm{CDCl}_{3}\right)$

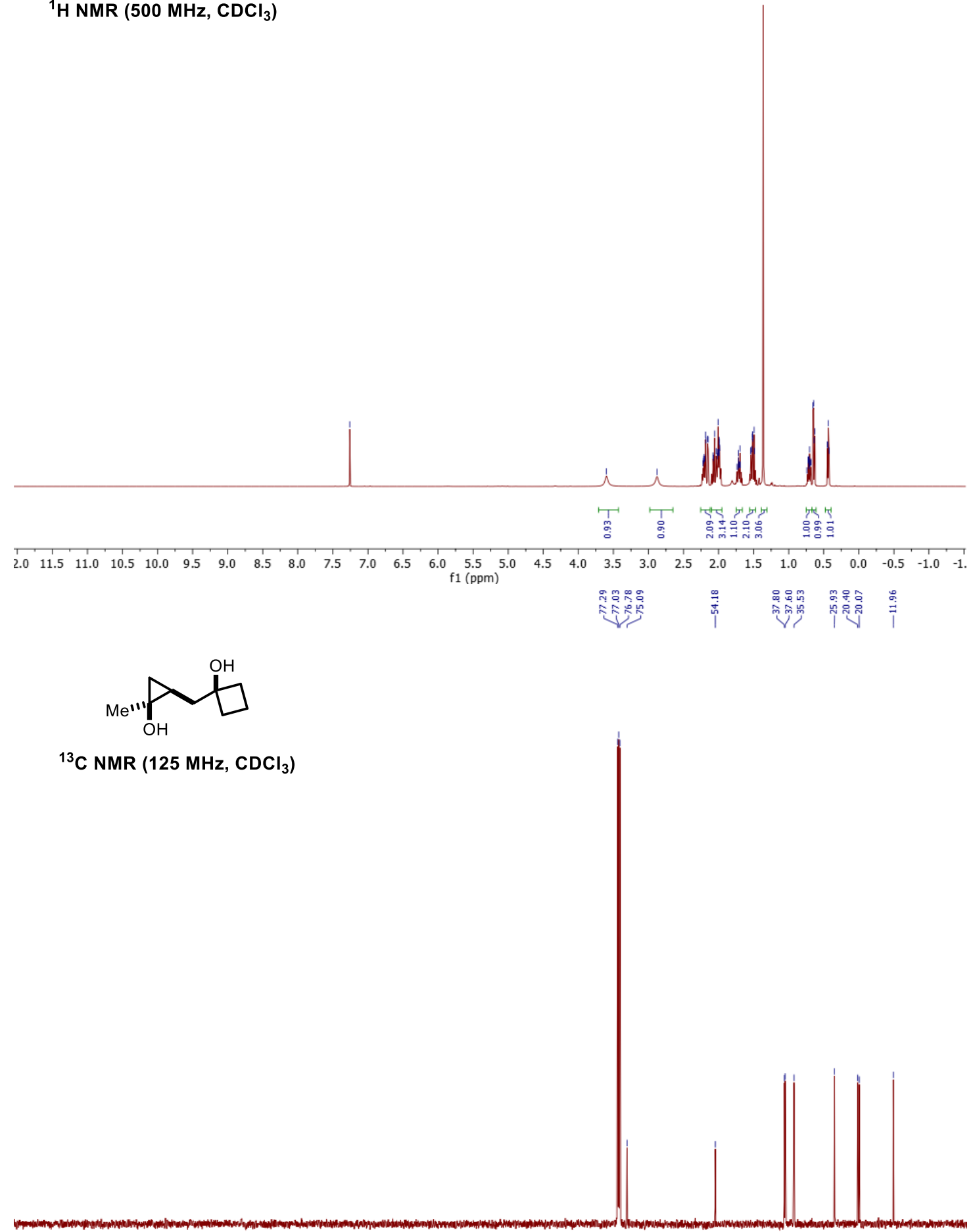

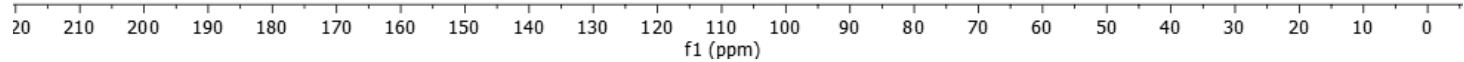




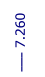

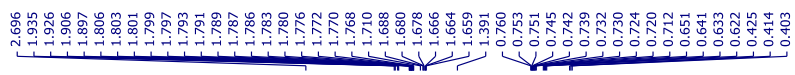
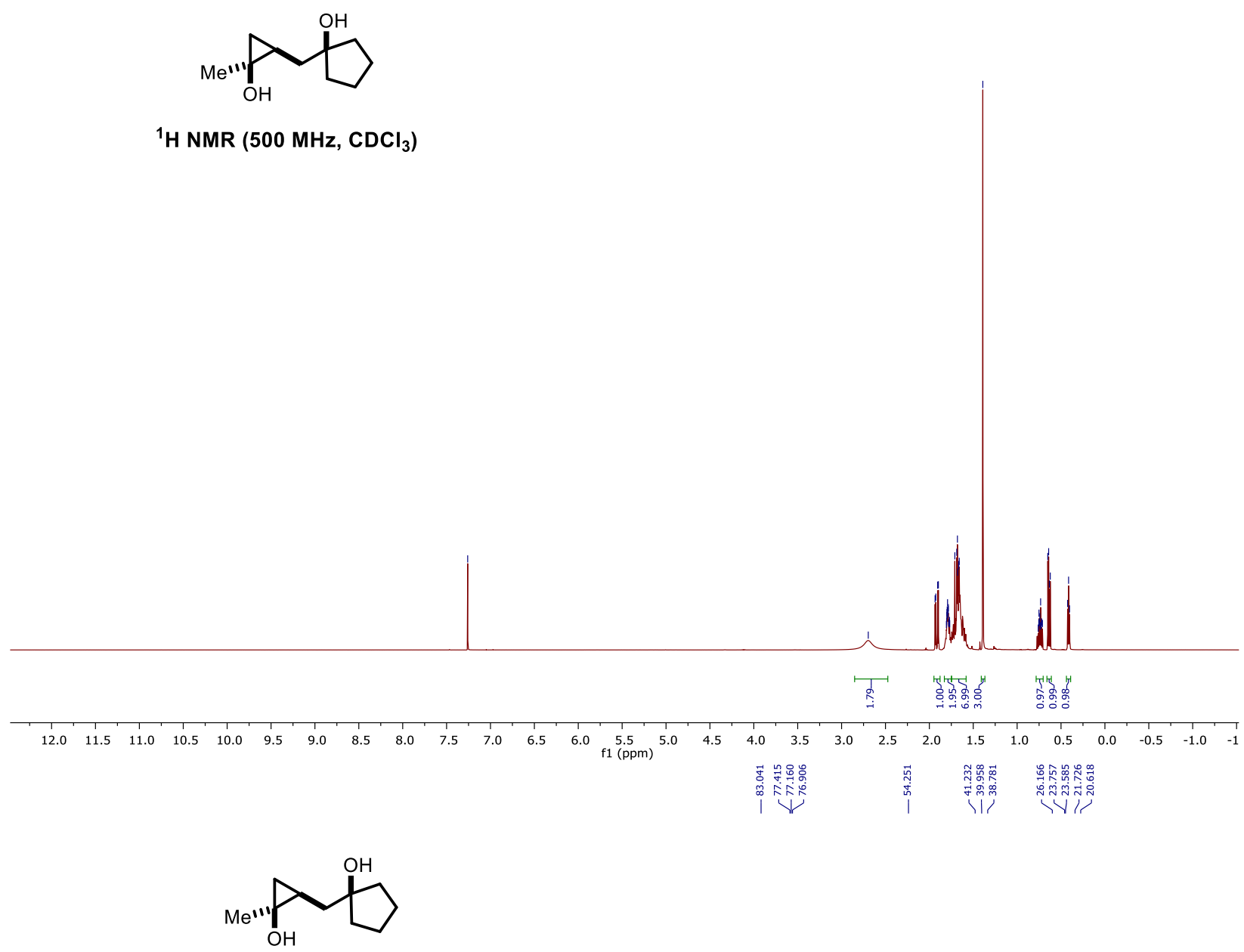

${ }^{13} \mathrm{C} \mathrm{NMR}\left(125 \mathrm{MHz}, \mathrm{CDCl}_{3}\right)$
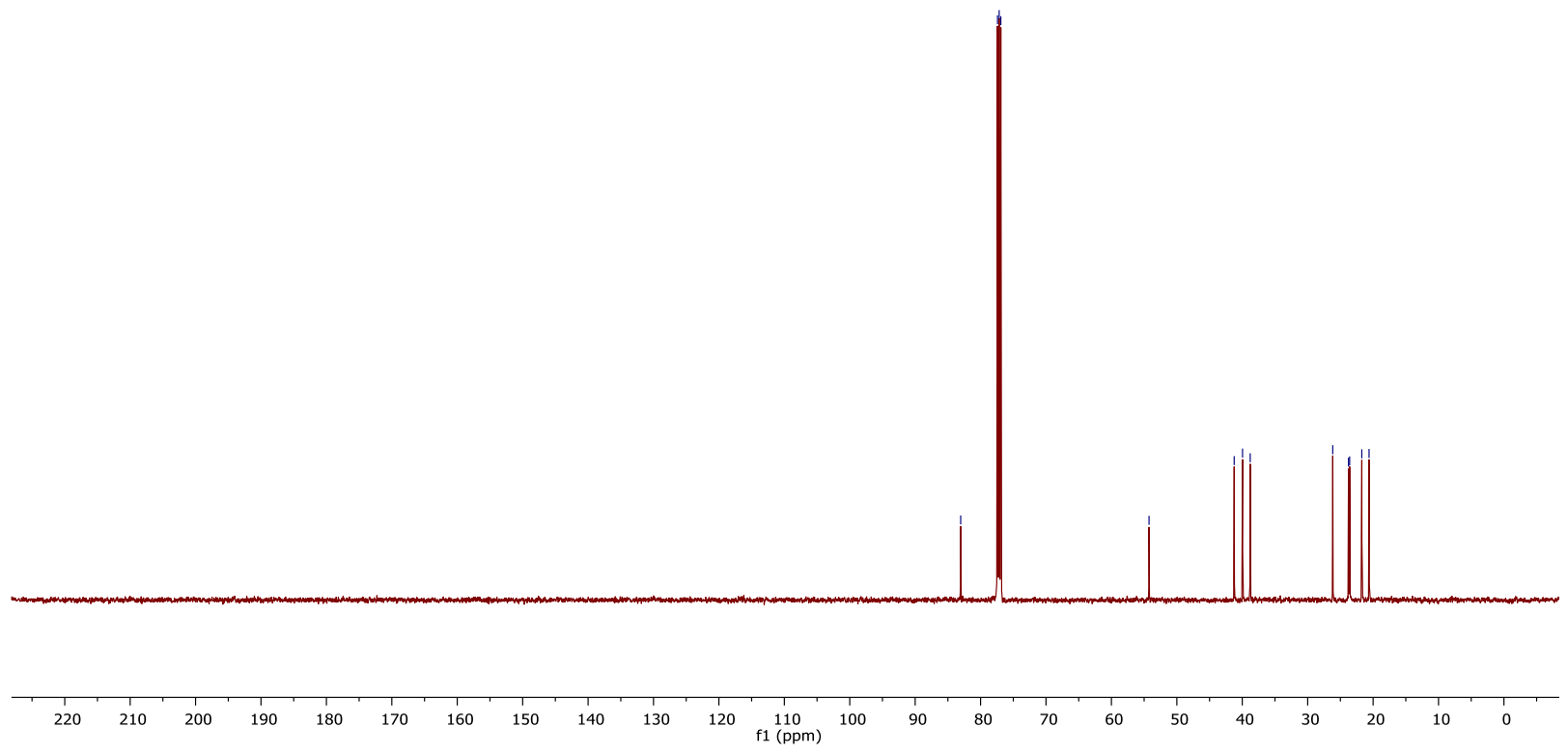

S52 


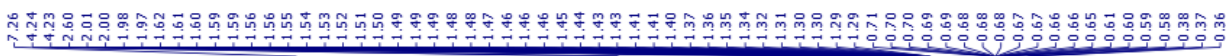

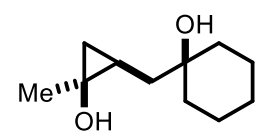

${ }^{1} \mathrm{H}$ NMR (500 MHz, $\left.\mathrm{CDCl}_{3}\right)$
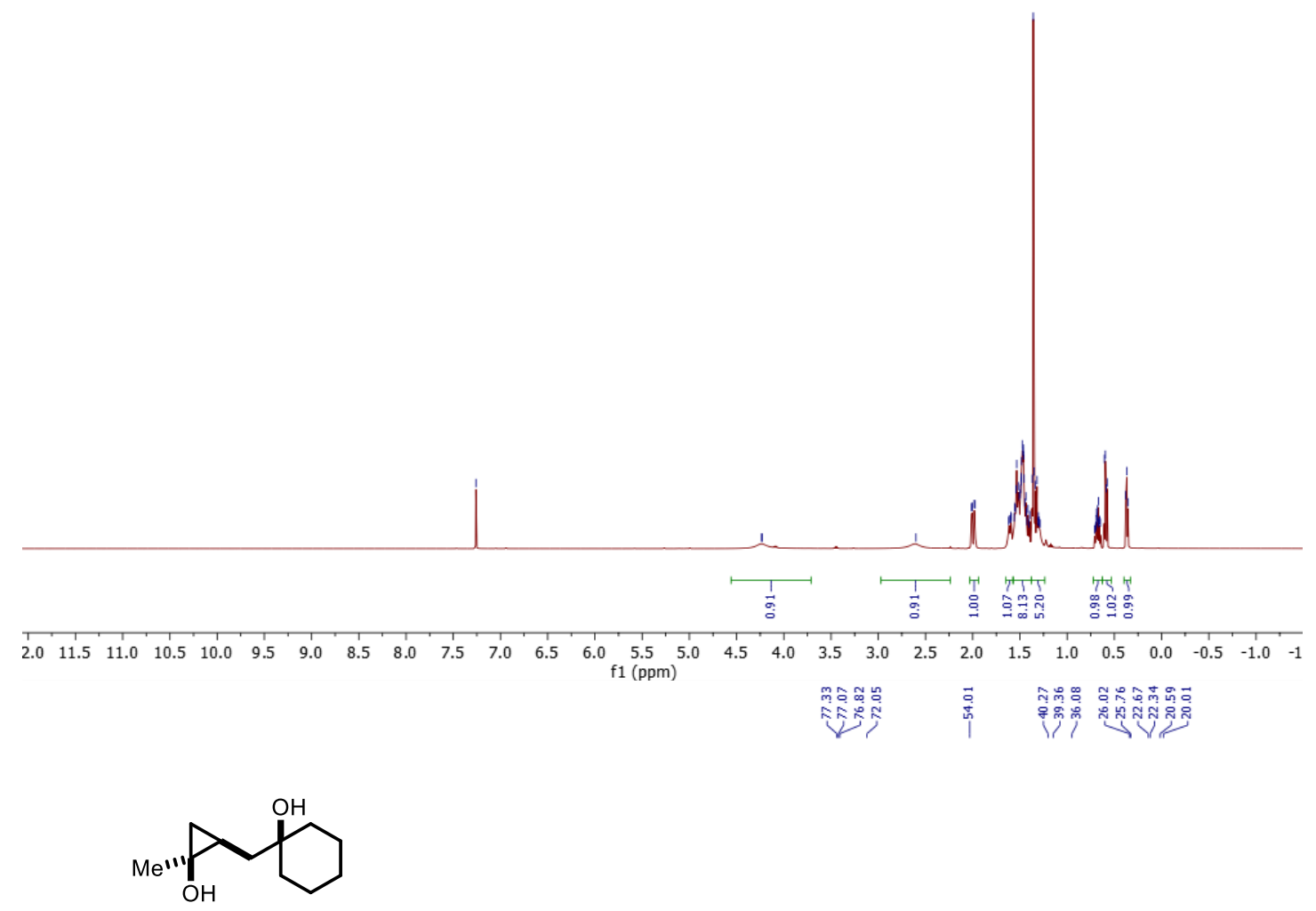

${ }^{13} \mathrm{C}$ NMR $\left(125 \mathrm{MHz}, \mathrm{CDCl}_{3}\right)$

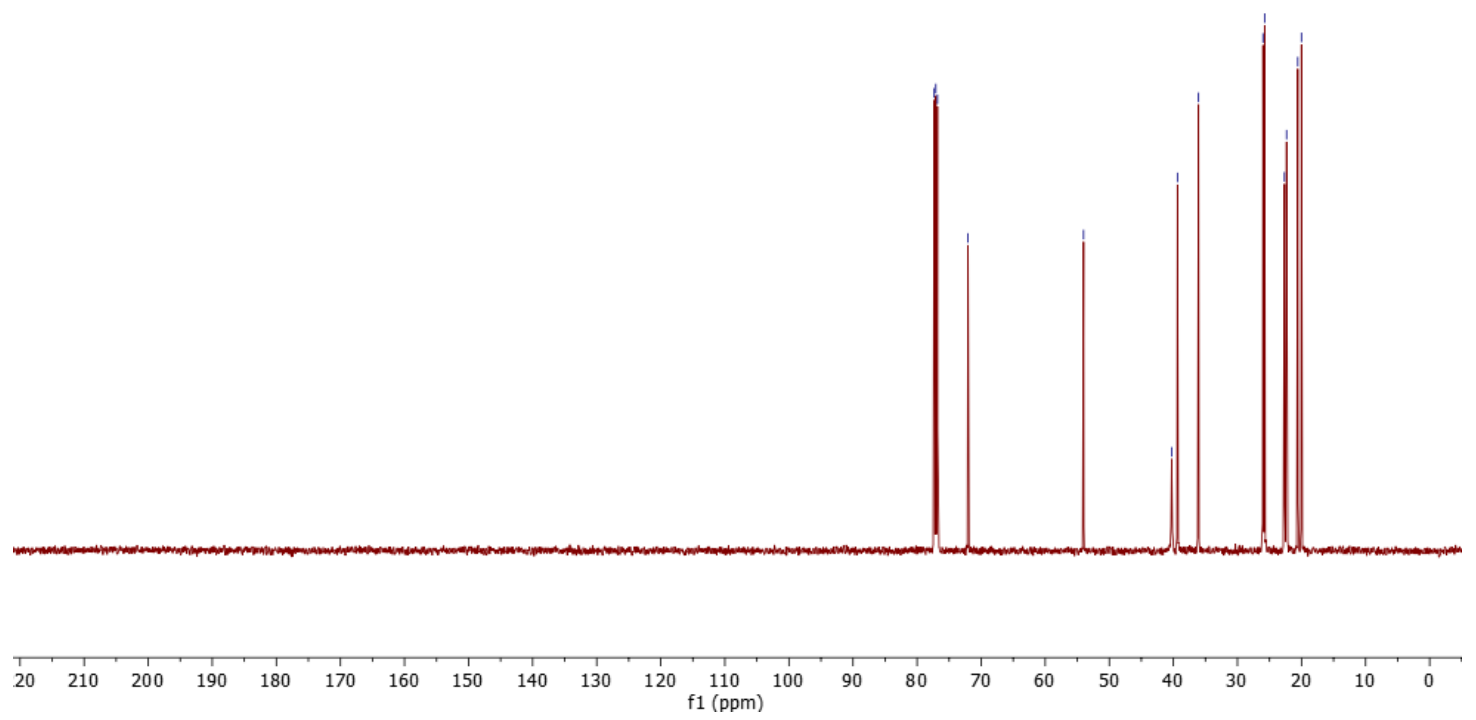

S53 
<smiles>C[C@]1(O)C[C@@H]1CC(O)(Cc1ccccc1)Cc1ccccc1</smiles>

${ }^{1} \mathrm{H}$ NMR (500 MHz, $\mathrm{CDCl}_{3}$ )
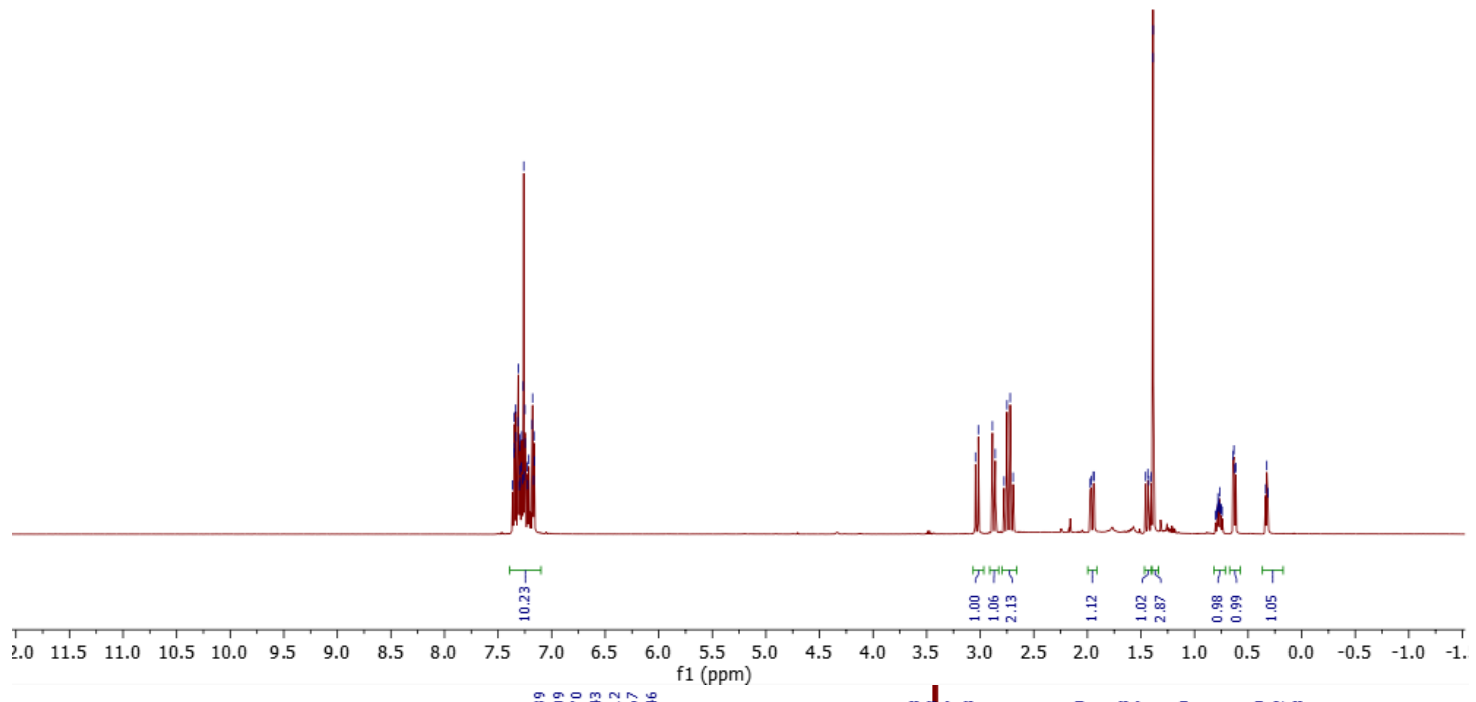

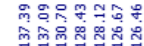

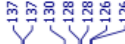<smiles>C[C@@]1(O)C[C@@H]1CC(O)(Cc1ccccc1)Cc1ccccc1</smiles>

${ }^{13} \mathrm{C}$ NMR (125 MHz, $\mathrm{CDCl}_{3}$ )

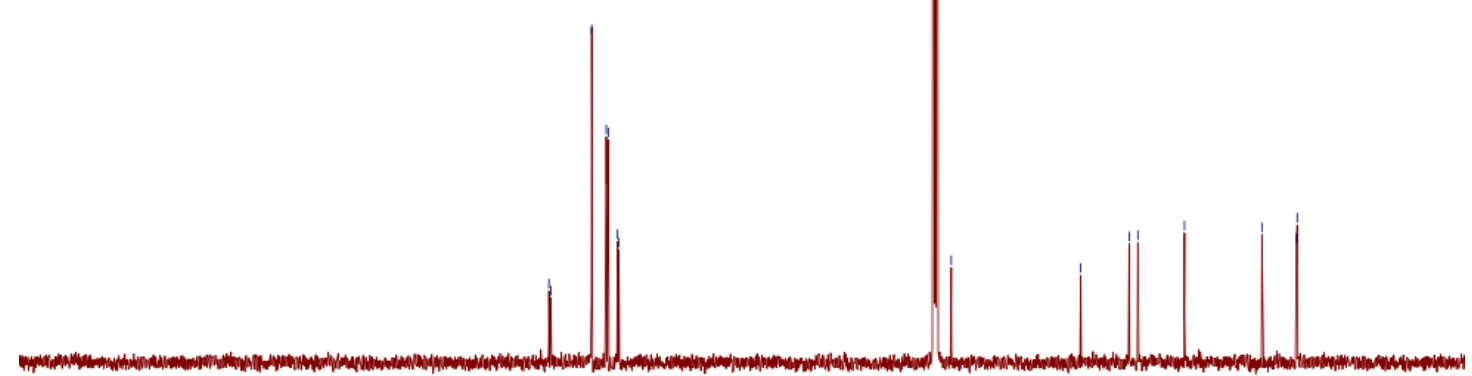

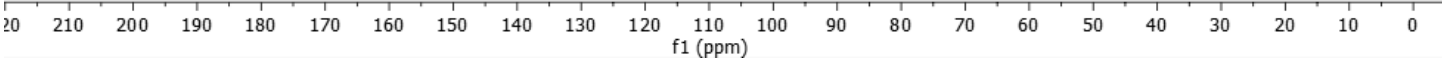



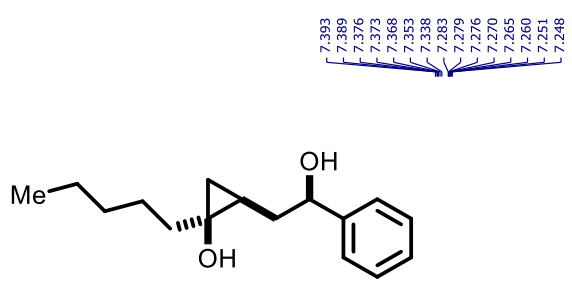

${ }^{1} \mathrm{H}$ NMR (500 MHz, $\mathrm{CDCl}_{3}$ )

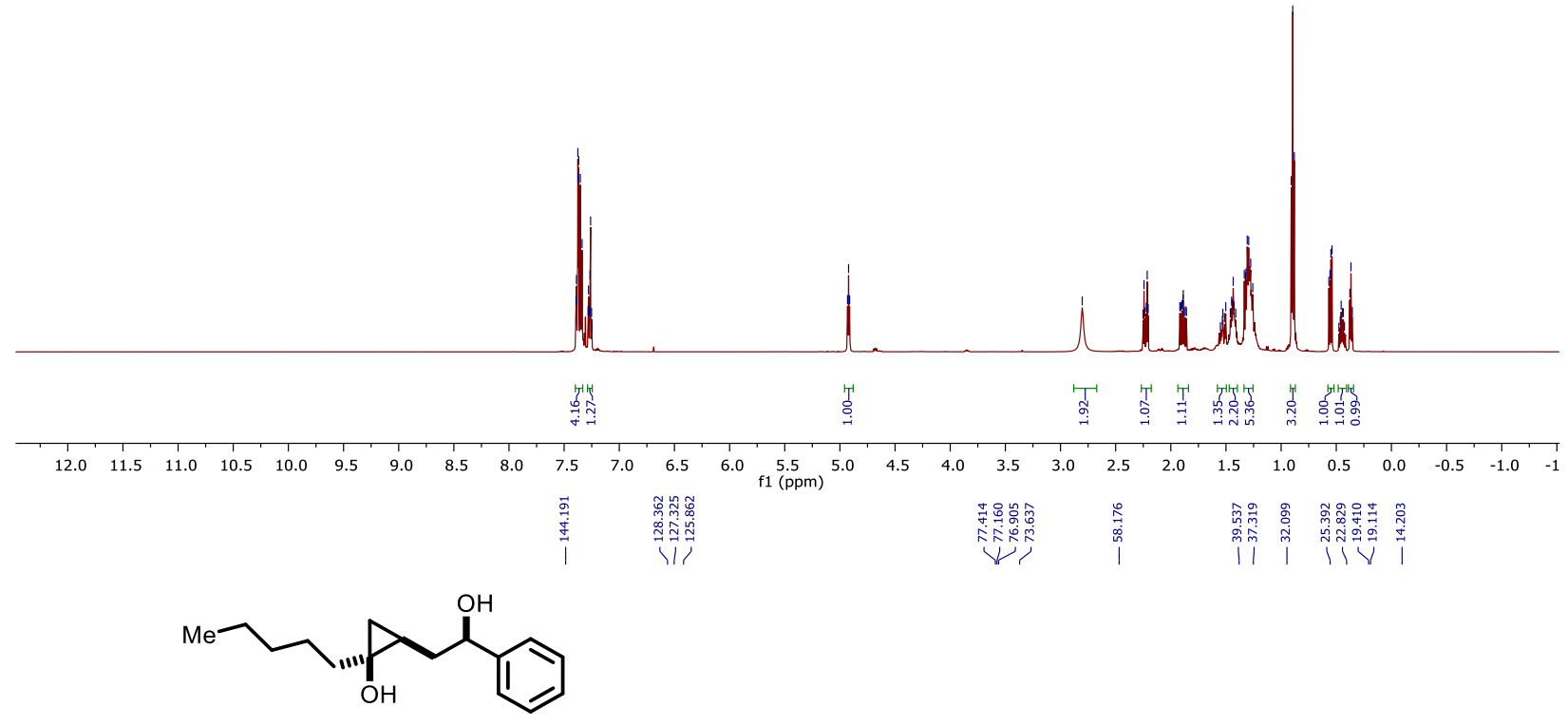

${ }^{13} \mathrm{C}$ NMR (125 MHz, $\mathrm{CDCl}_{3}$ )
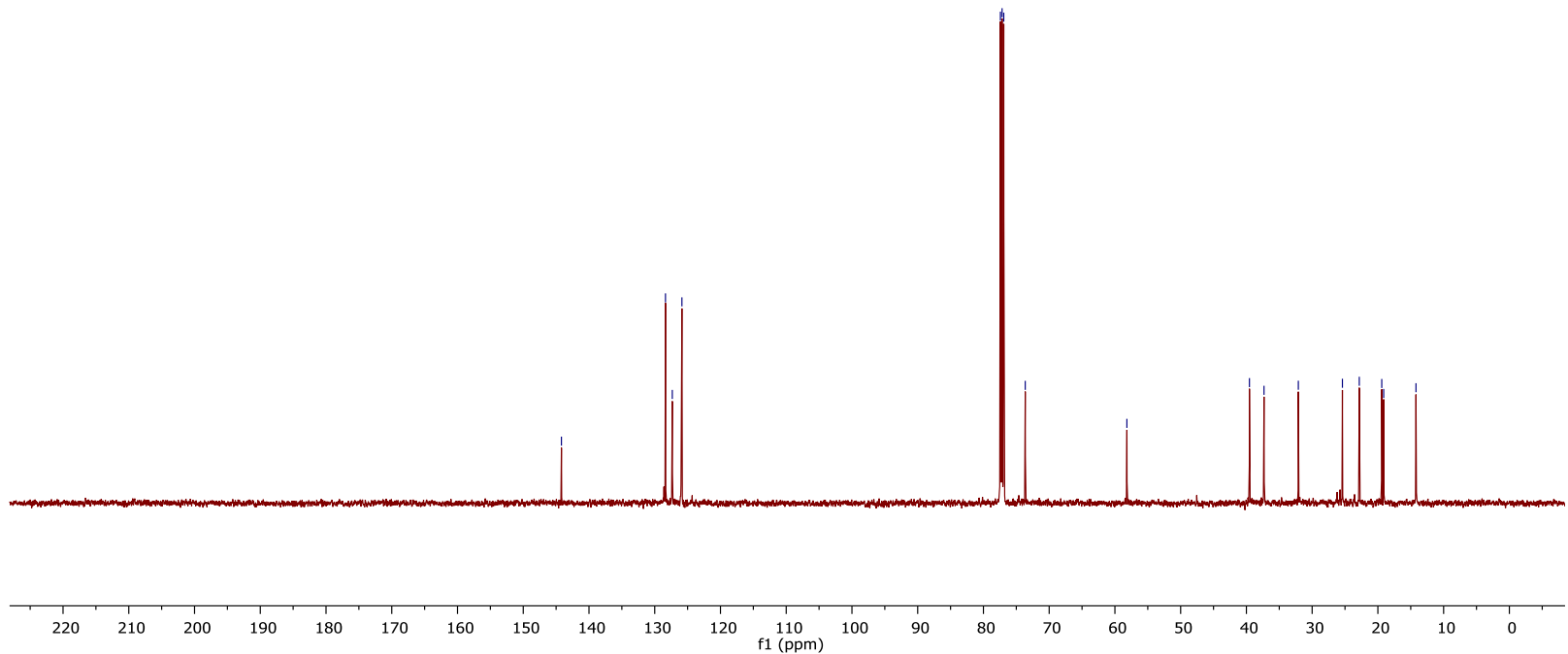


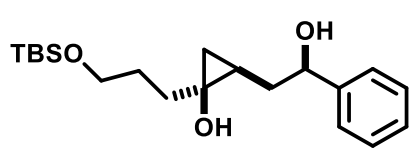

${ }^{1} \mathrm{H}$ NMR (500 MHz, $\mathrm{CDCl}_{3}$ )
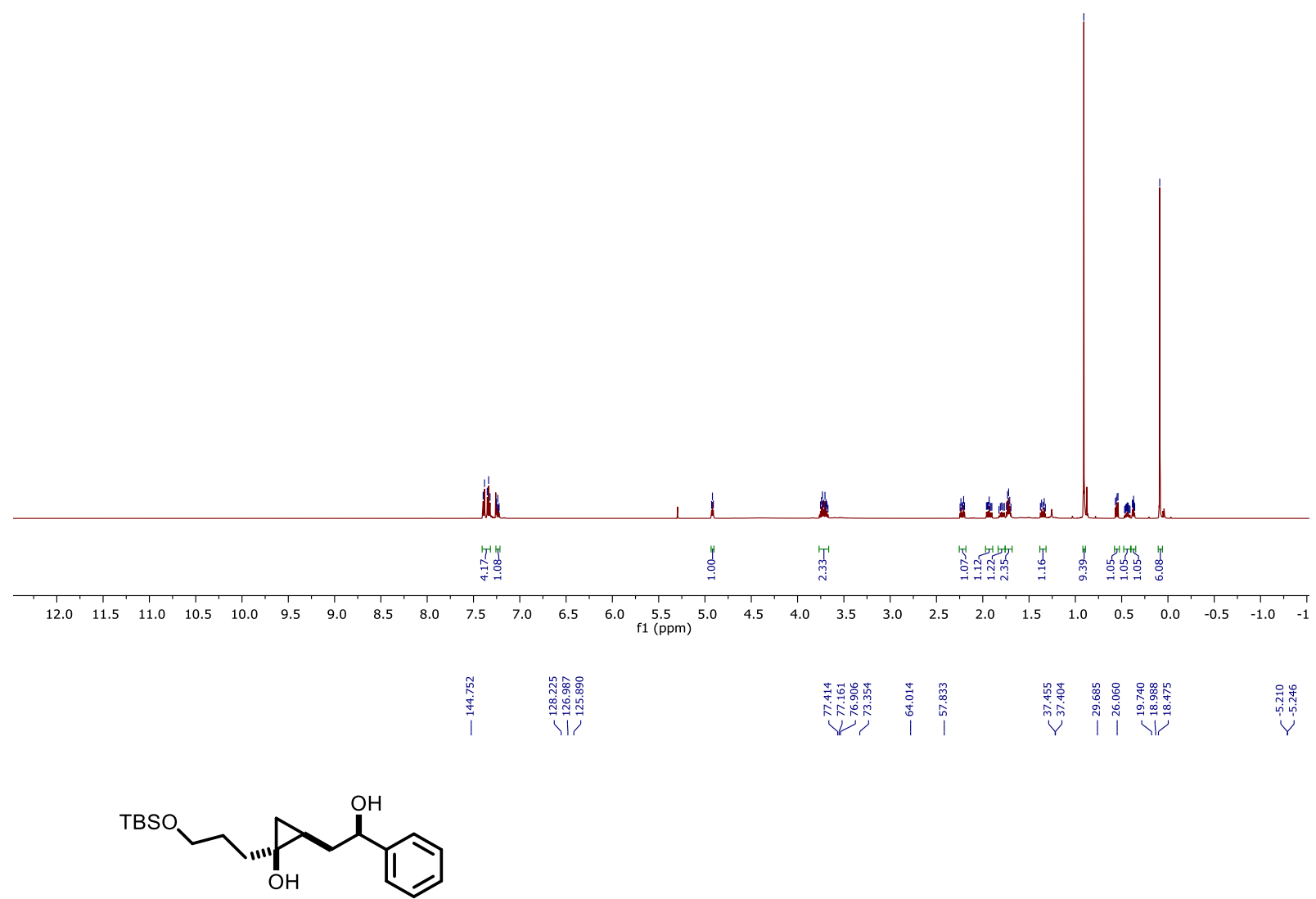

${ }^{13} \mathrm{C}$ NMR $\left(125 \mathrm{MHz}, \mathrm{CDCl}_{3}\right)$

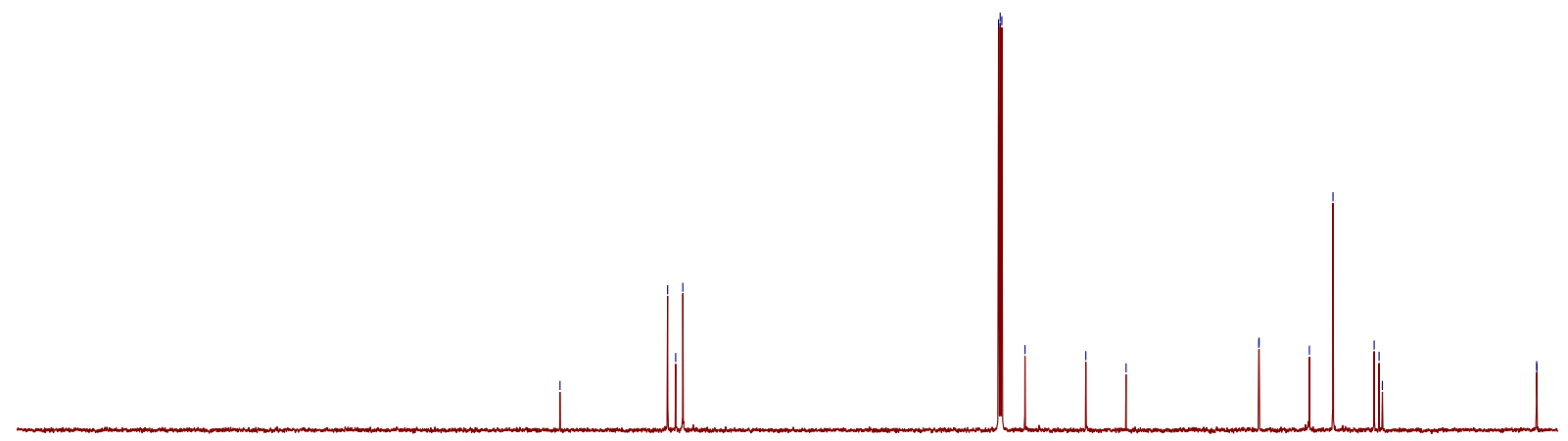

$\begin{array}{lllllllllllllllllllllllllll}220 & 210 & 200 & 190 & 180 & 170 & 160 & 150 & 140 & 130 & 120 & 110 & 100 & 90 & 80 & 70 & 60 & 50 & 40 & 30 & 20 & 10 & 0\end{array}$ 


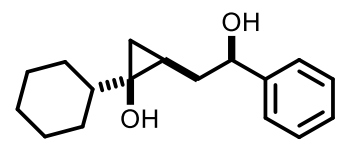

${ }^{1} \mathrm{H}$ NMR (500 MHz, $\left.\mathrm{CDCl}_{3}\right)$
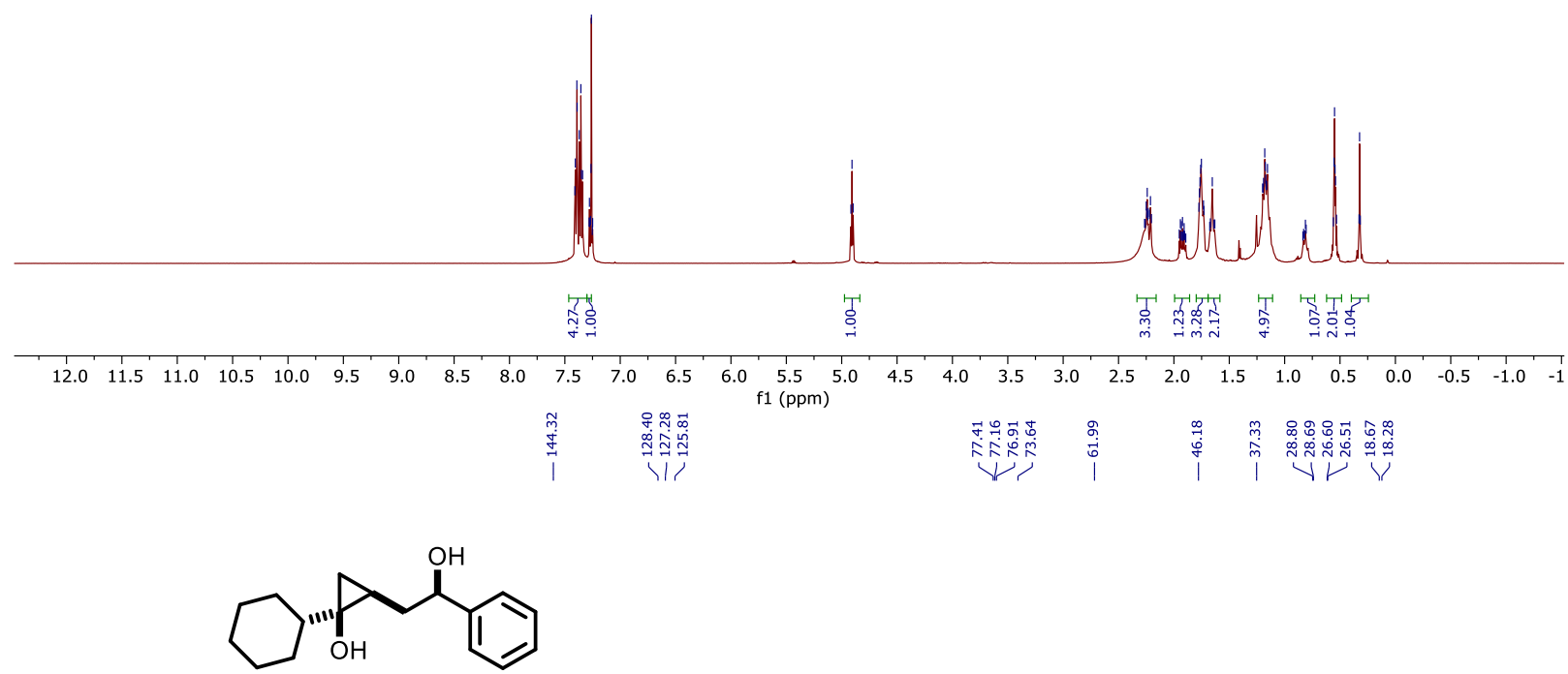

${ }^{13} \mathrm{C}$ NMR $\left(125 \mathrm{MHz}, \mathrm{CDCl}_{3}\right)$

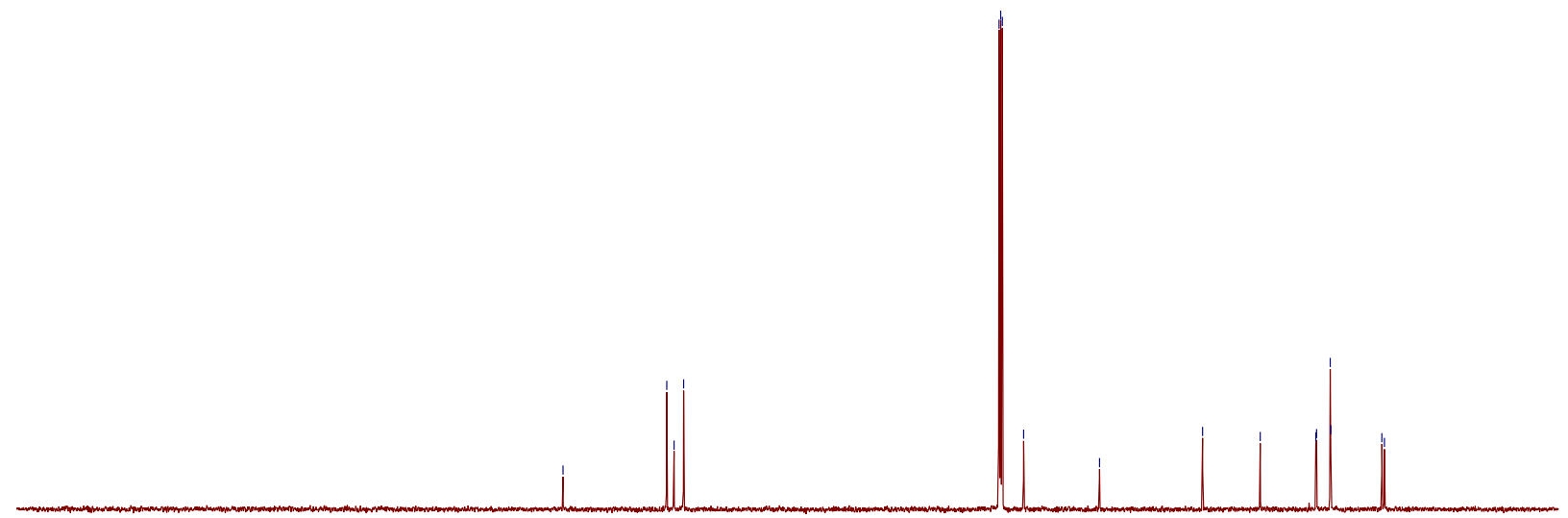

$\begin{array}{lllllllllllllllllllllllllll}220 & 210 & 200 & 190 & 180 & 170 & 160 & 150 & 140 & 130 & 120 & 110 & 100 & 90 & 80 & 70 & 60 & 50 & 40 & 30 & 20 & 10 & 0\end{array}$ 
<smiles>OC(CC1C[C@]1(O)Cc1ccccc1)c1ccccc1</smiles>

${ }^{1} \mathrm{H}$ NMR (500 MHz, $\mathrm{CDCl}_{3}$ )
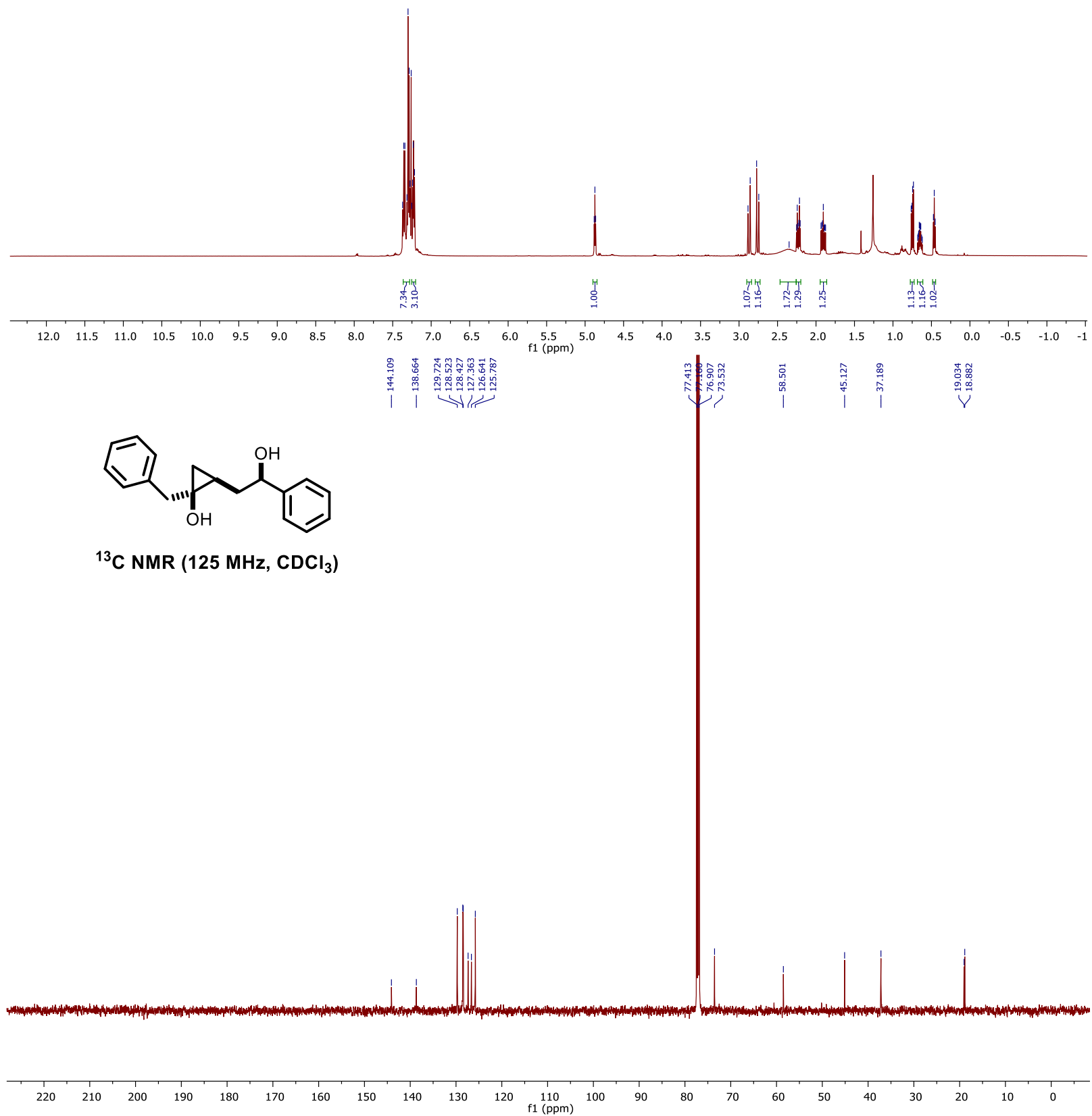


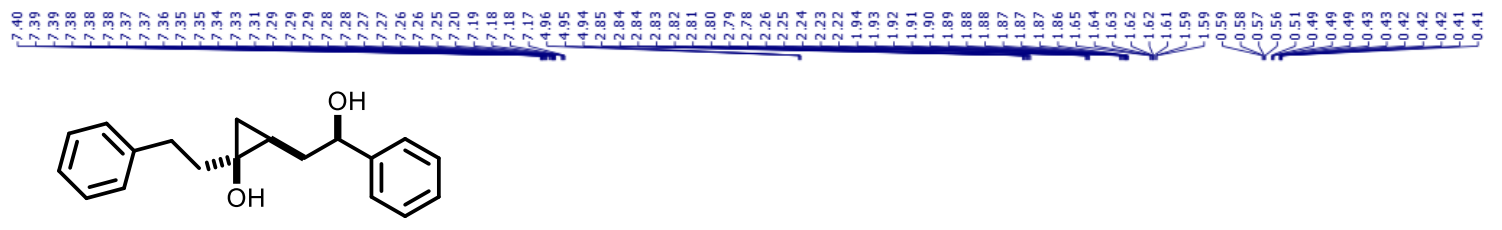

${ }^{1} \mathrm{H}$ NMR (500 MHz, $\left.\mathrm{CDCl}_{3}\right)$

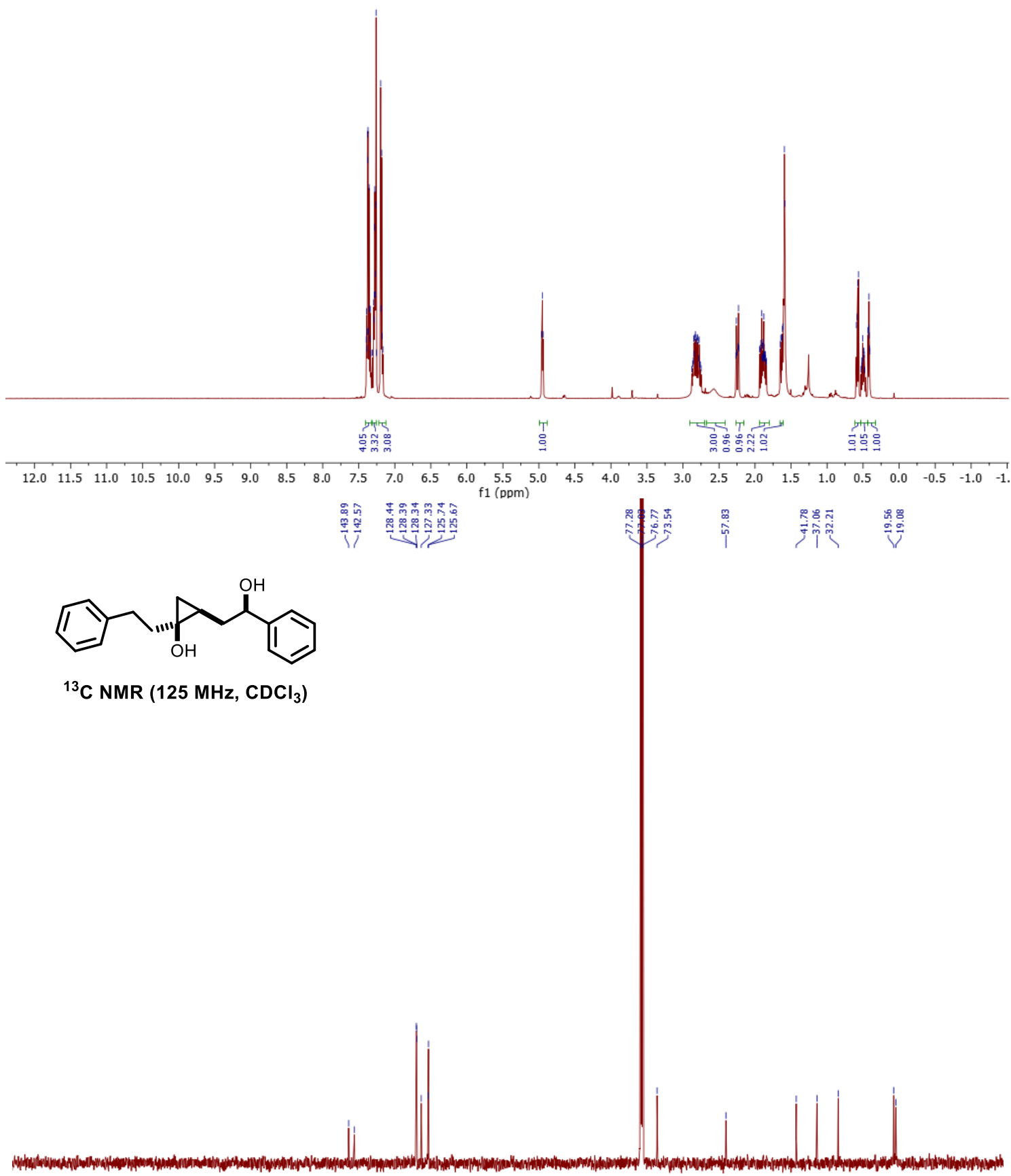

\begin{tabular}{lllllllllllllllllllllllllllll}
\hline 20 & 210 & 200 & 190 & 180 & 170 & 160 & 150 & 140 & 130 & 120 & 110 & 100 & 90 & 80 & 70 & 60 & 50 & 40 & 30 & 20 & 10 & 0
\end{tabular} 

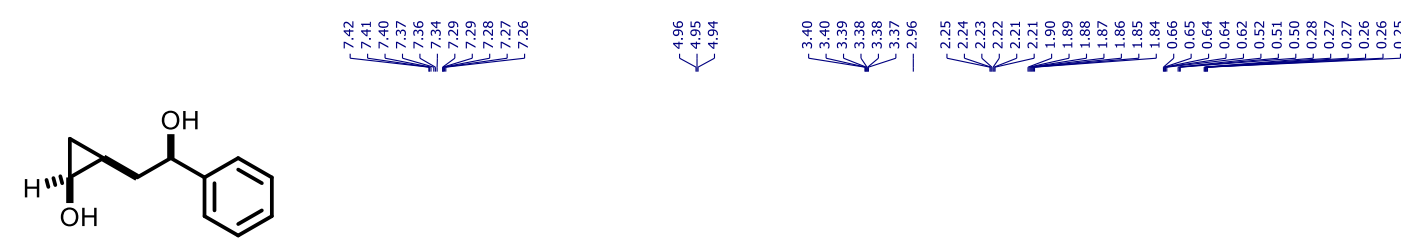

${ }^{1} \mathrm{H}$ NMR $\left(500 \mathrm{MHz}, \mathrm{CDCl}_{3}\right)$

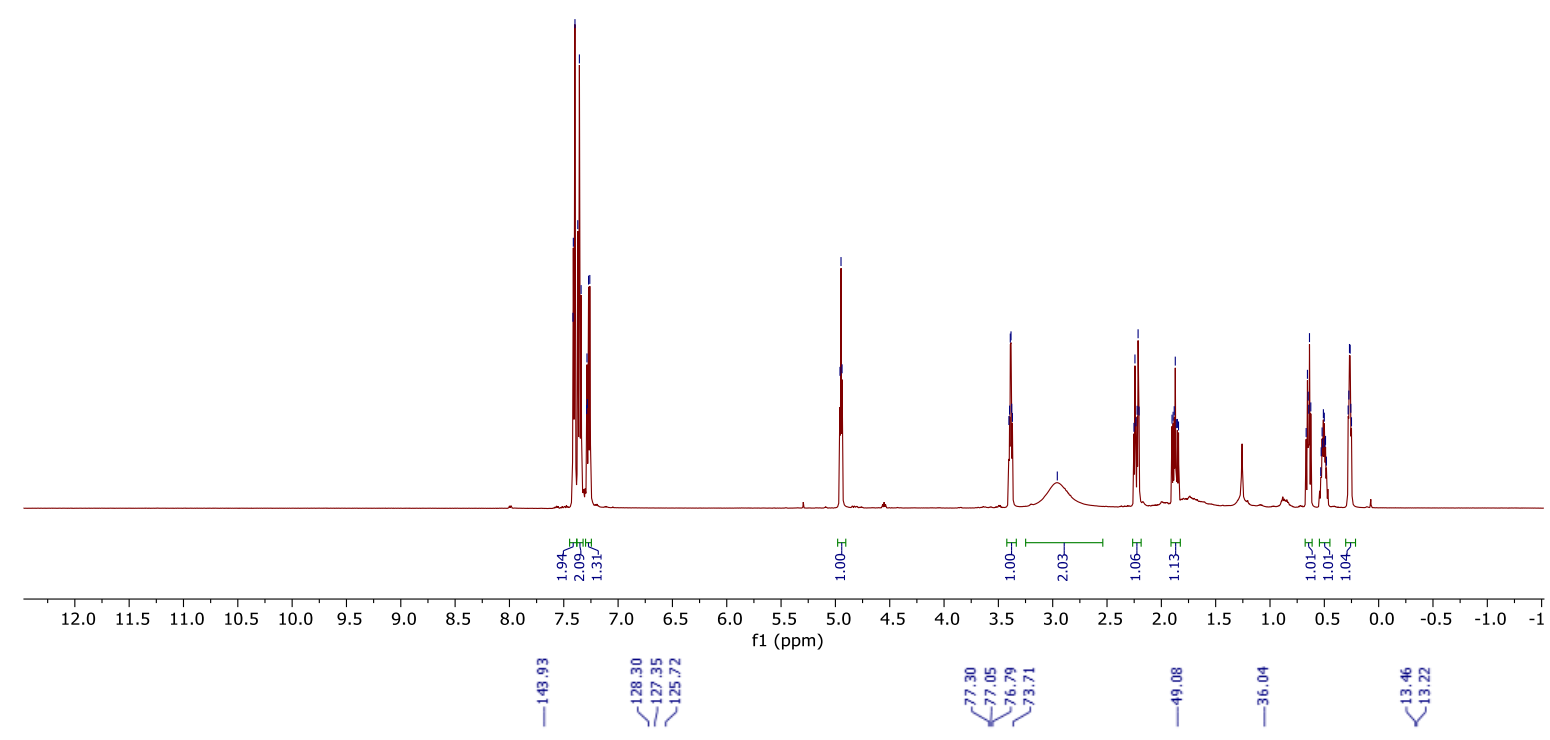<smiles>OC(C[C@@H]1C[C@H]1O)c1ccccc1</smiles>

${ }^{13} \mathrm{C}$ NMR $\left(125 \mathrm{MHz}, \mathrm{CDCl}_{3}\right)$

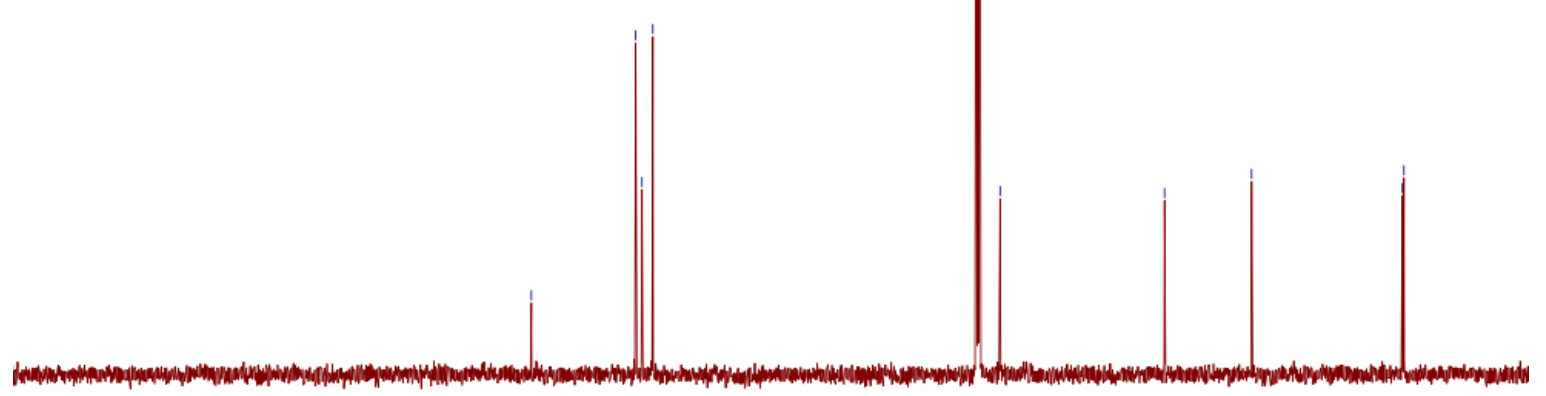

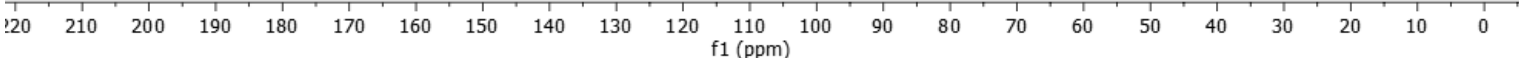




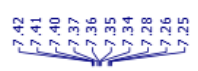

畒前<smiles>CC(C)[C@]1(O)CC1CC(O)c1ccccc1</smiles>

${ }^{1} \mathrm{H}$ NMR (500 MHz, $\left.\mathrm{CDCl}_{3}\right)$

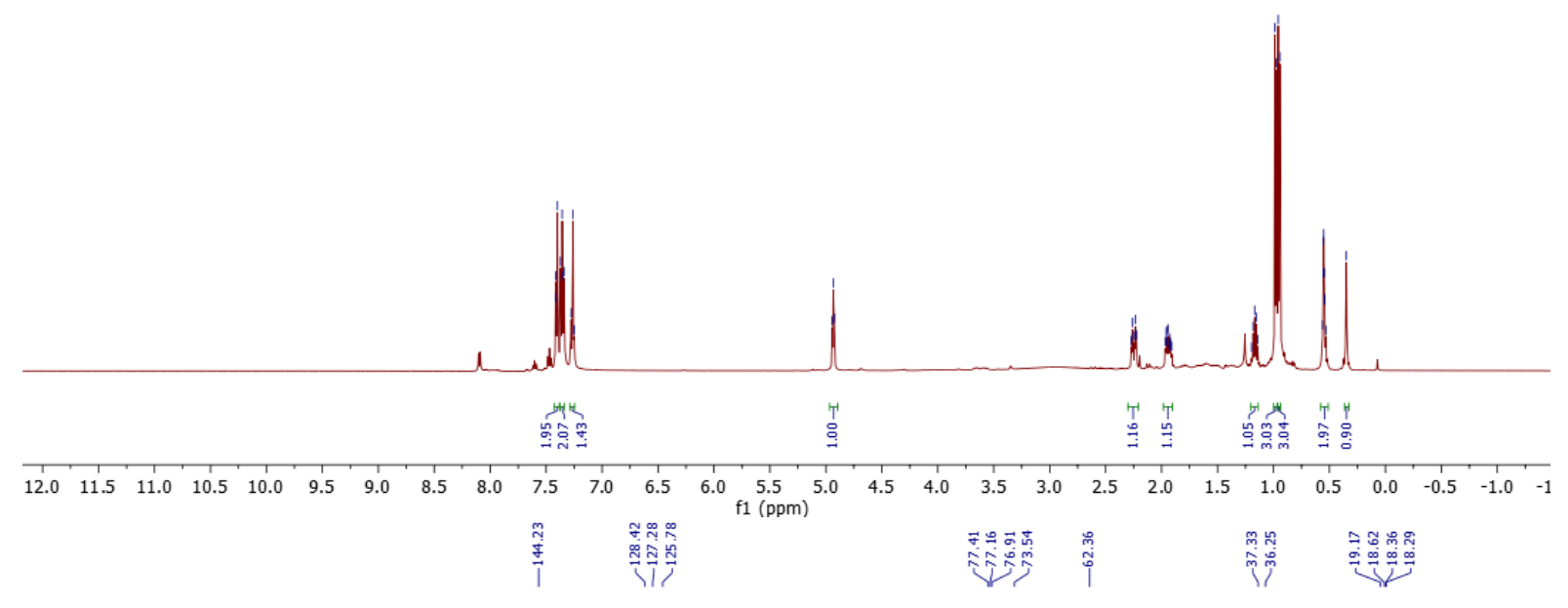<smiles>CC(C)[C@]1(O)CC1CC(O)c1ccccc1</smiles>

${ }^{13} \mathrm{C}$ NMR (125 MHz, $\left.\mathrm{CDCl}_{3}\right)$
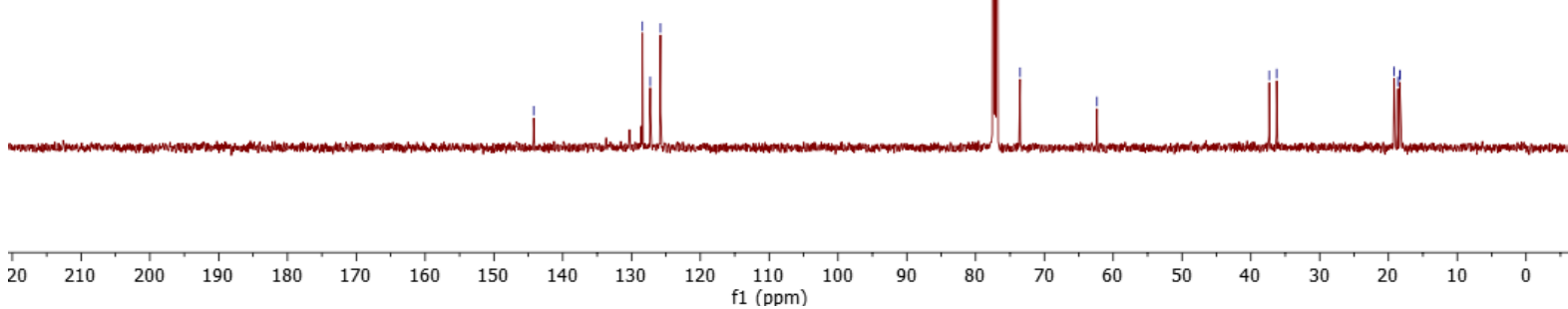

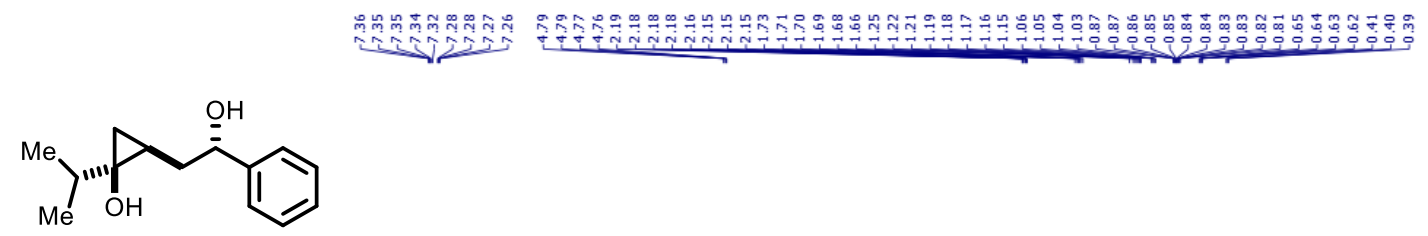

${ }^{1} \mathrm{H}$ NMR $\left(500 \mathrm{MHz}, \mathrm{CDCl}_{3}\right)$

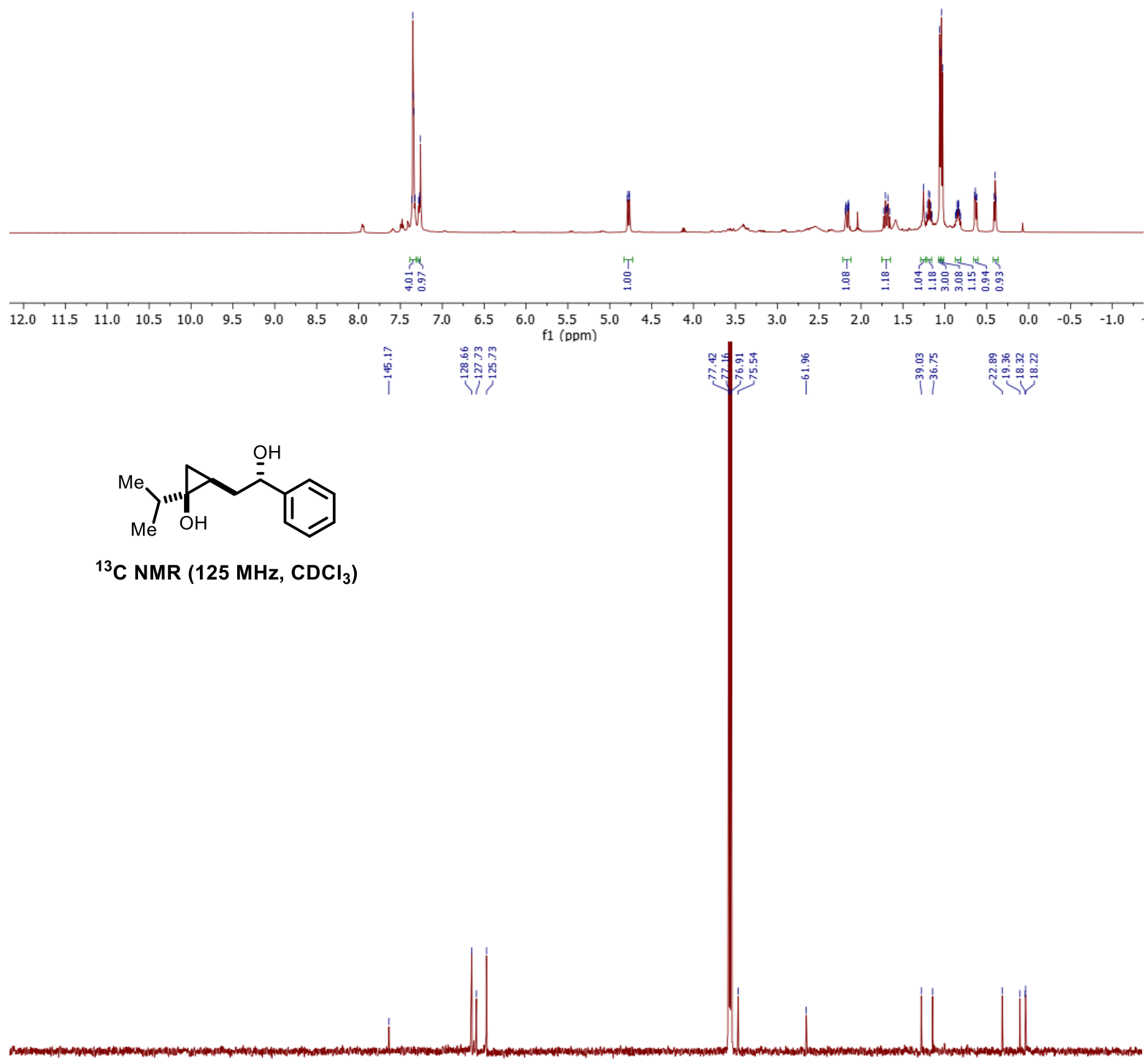

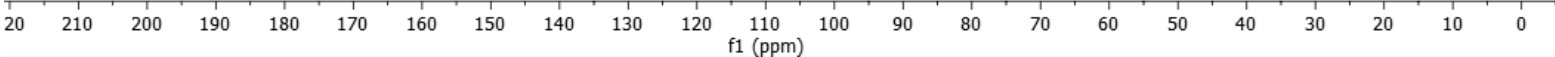


<smiles>C[C@@]1(O)CC1CCC1(O)CCC1</smiles>

${ }^{1} \mathrm{H}$ NMR (500 MHz, $\mathrm{CDCl}_{3}$ )

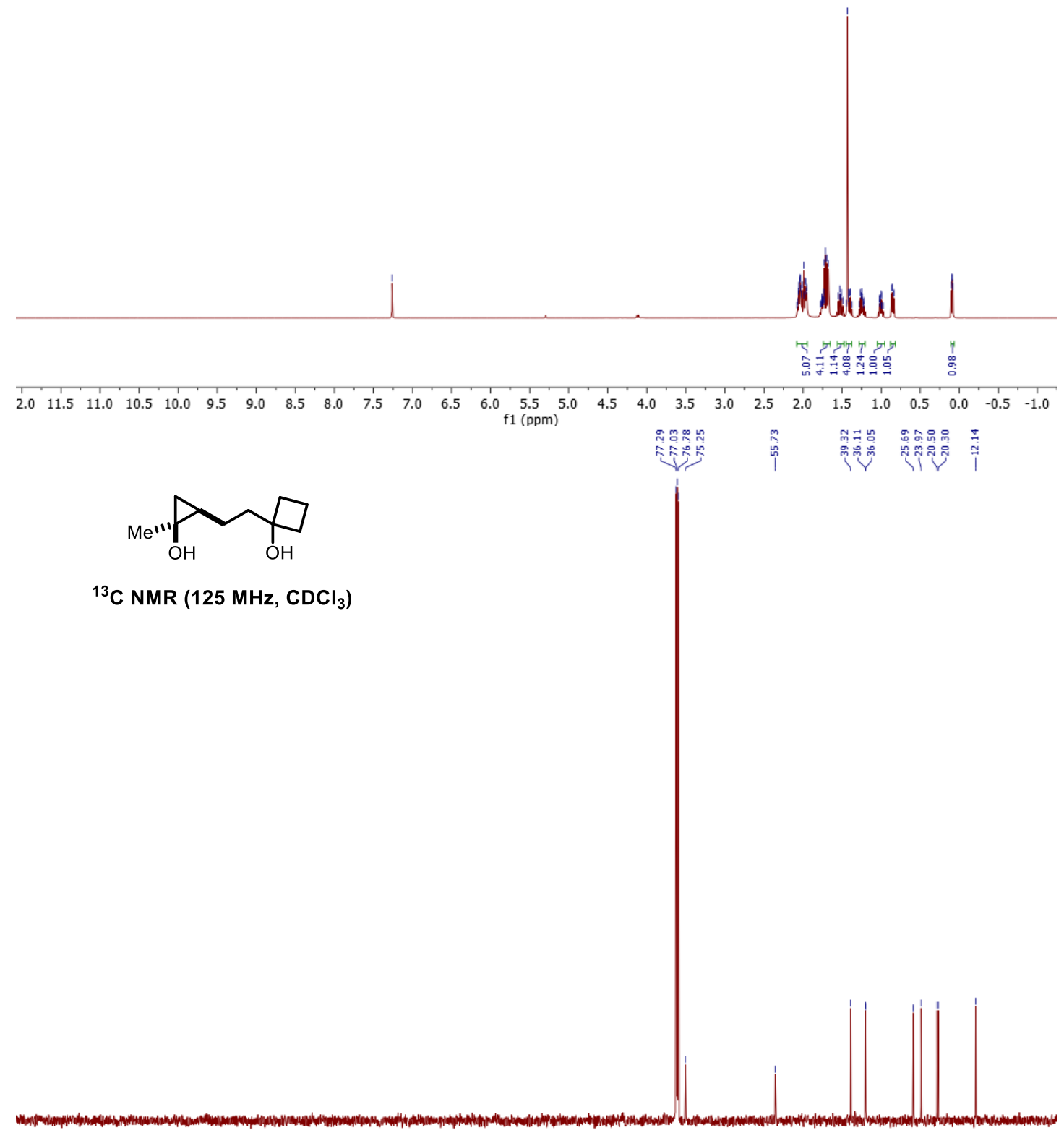

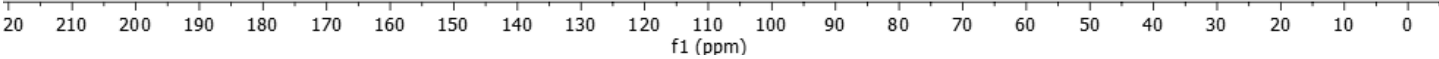




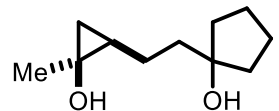

${ }^{1} \mathrm{H}$ NMR (500 MHz, $\left.\mathrm{CDCl}_{3}\right)$

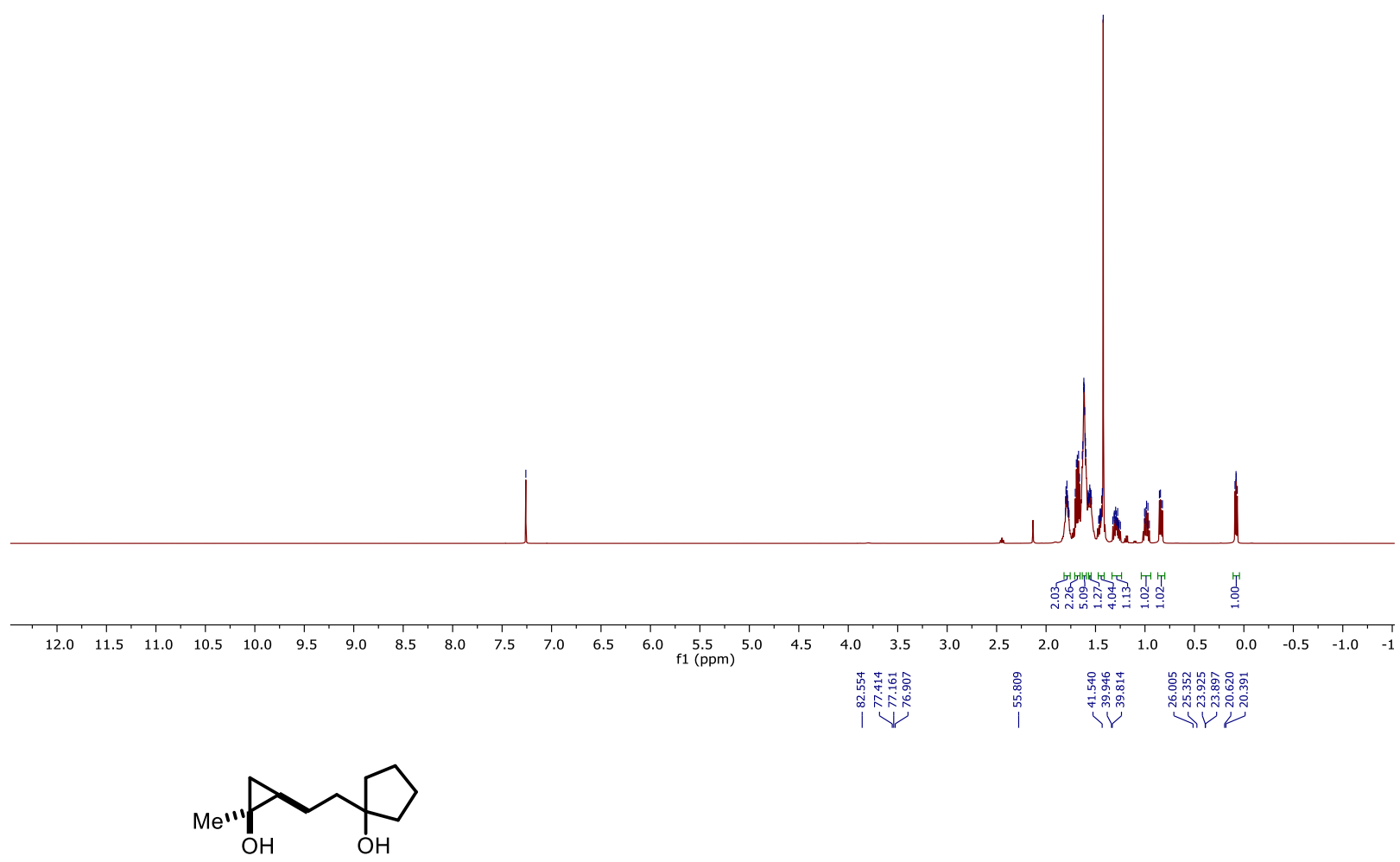

${ }^{1} \mathrm{H}$ NMR (500 MHz, $\left.\mathrm{CDCl}_{3}\right)$

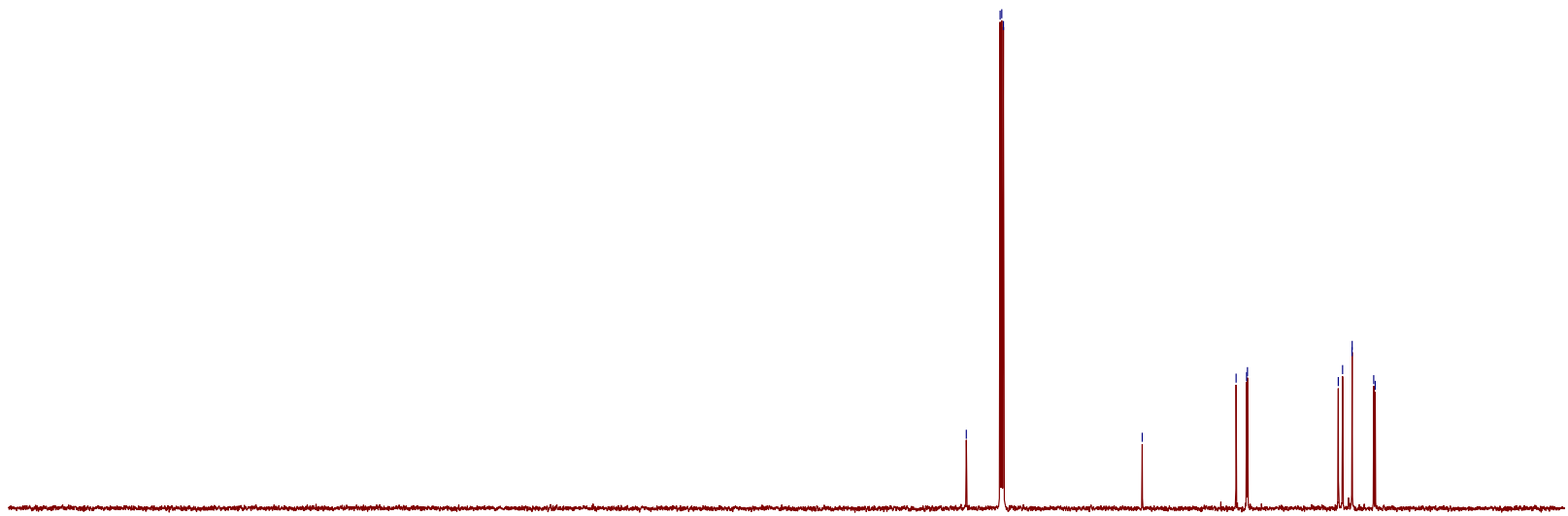

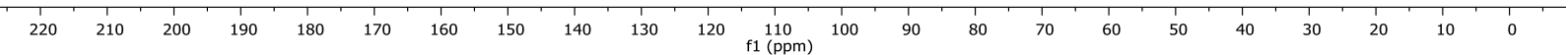


<smiles>C[C@@]1(O)C[C@@H]1CCC1(O)CCCCC1</smiles>

${ }^{1} \mathrm{H}$ NMR $\left(500 \mathrm{MHz}, \mathrm{CDCl}_{3}\right)$

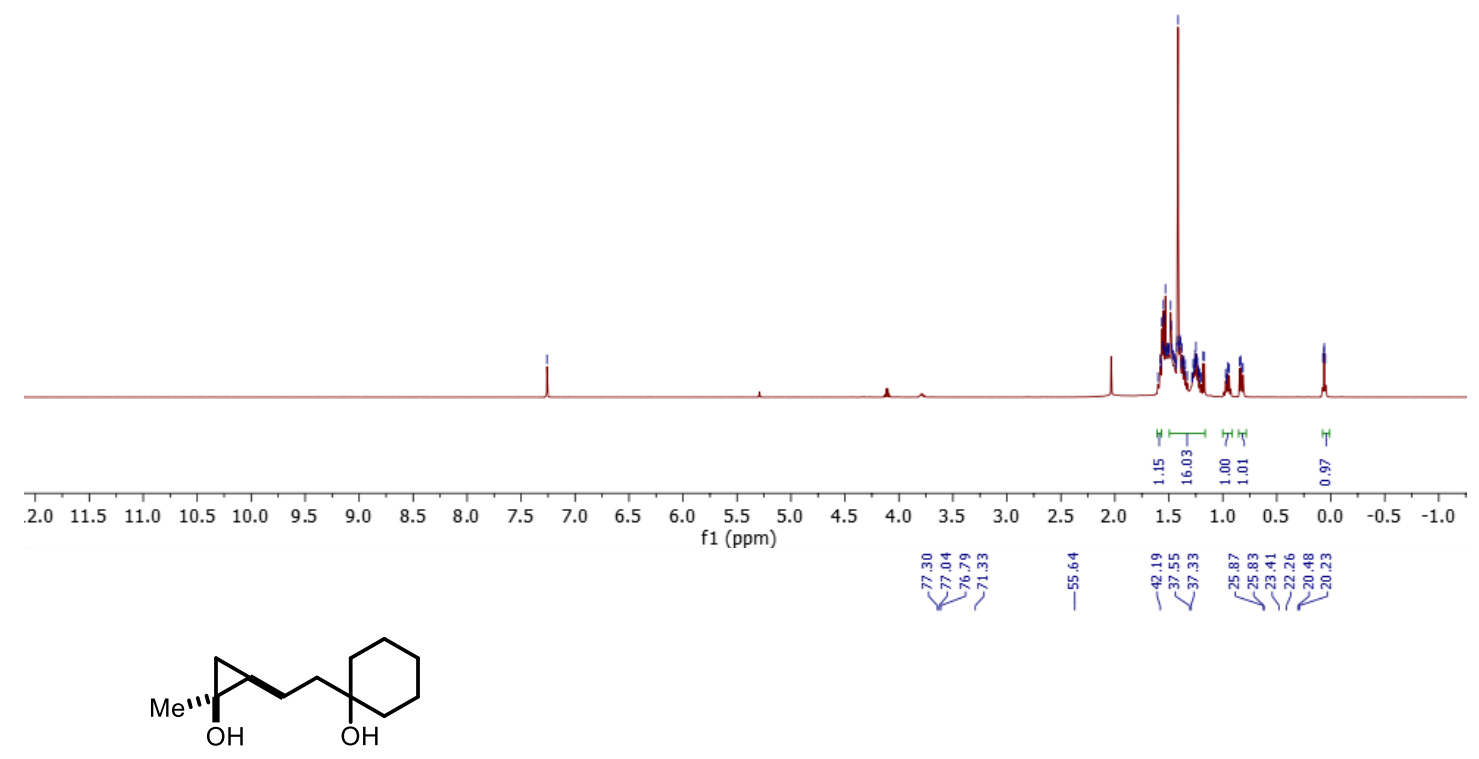

${ }^{13} \mathrm{C}$ NMR $\left(125 \mathrm{MHz}, \mathrm{CDCl}_{3}\right)$

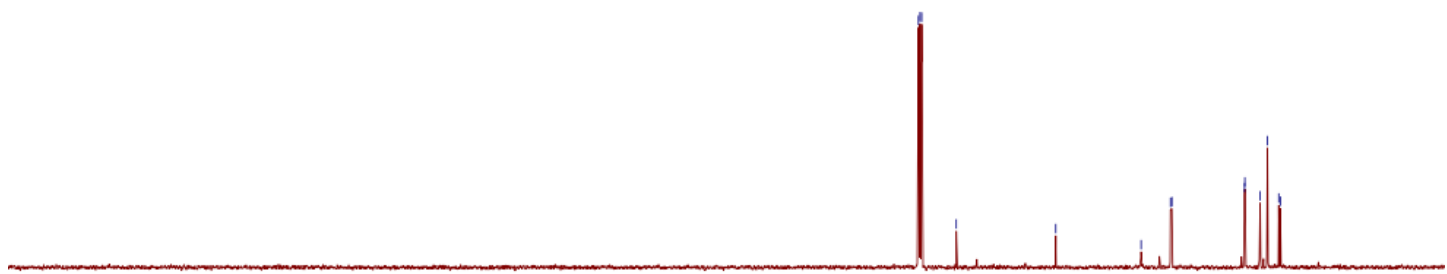

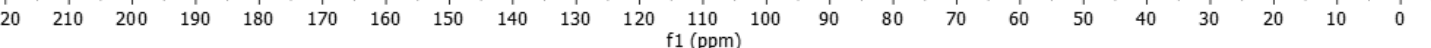


<smiles>C[C@@]1(O)CC1CCC[C@H](O)c1ccccc1</smiles>

${ }^{1} \mathrm{H}$ NMR (500 MHz, $\left.\mathrm{CDCl}_{3}\right)$

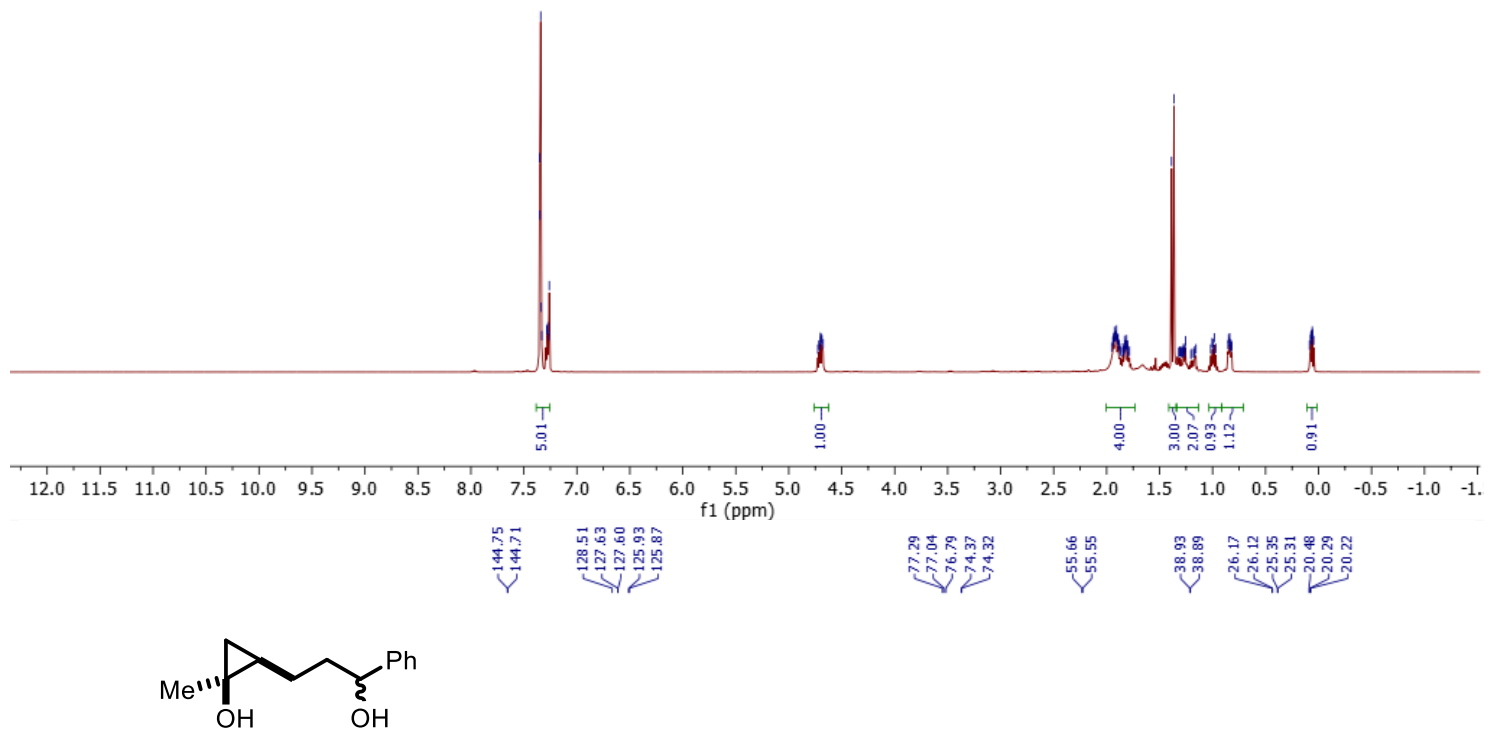

${ }^{13} \mathrm{C}$ NMR $\left(125 \mathrm{MHz}, \mathrm{CDCl}_{3}\right)$

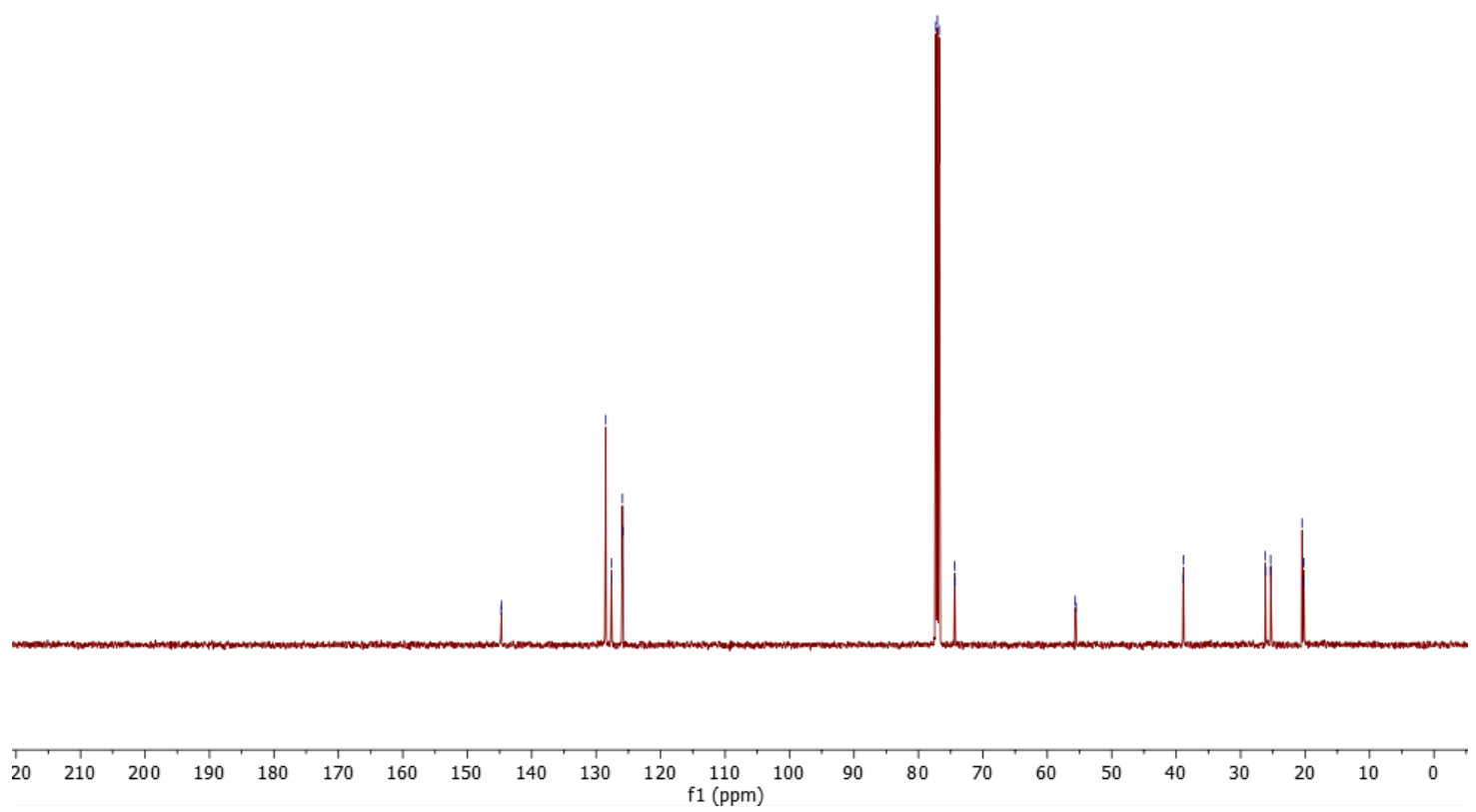


<smiles>OCCC[C@]1(O)CC1CC(O)c1ccccc1</smiles>

${ }^{1} \mathrm{H}$ NMR (500 MHz, $\mathrm{CDCl}_{3}$ )

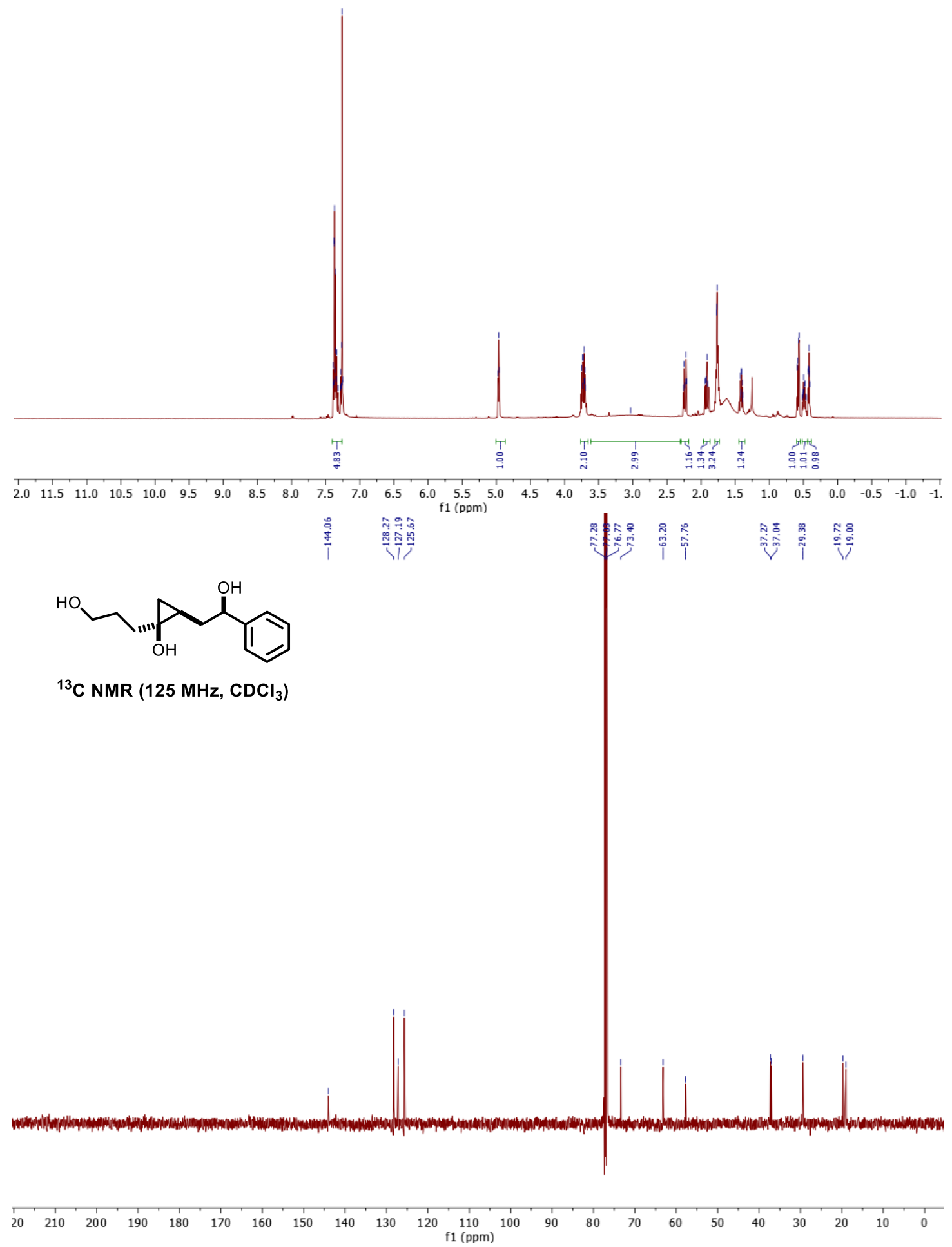




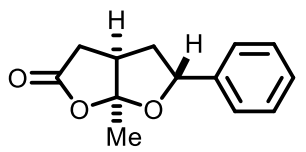

${ }^{1} \mathrm{H}$ NMR $\left(500 \mathrm{MHz}, \mathrm{CDCl}_{3}\right)$

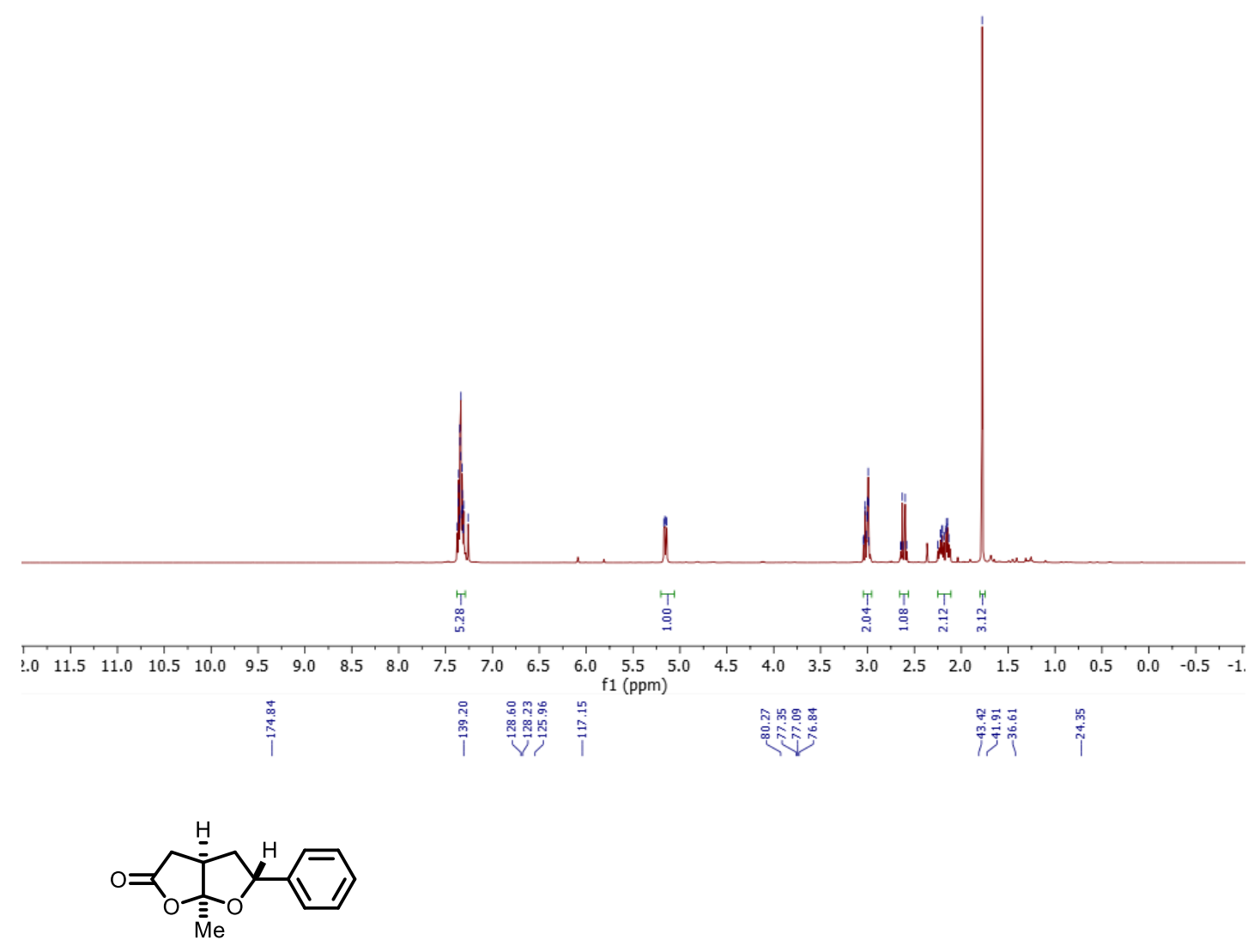

$\left.{ }^{13} \mathrm{C} \mathrm{NMR} \mathrm{(125} \mathrm{MHz,} \mathrm{CDCl}_{3}\right)$

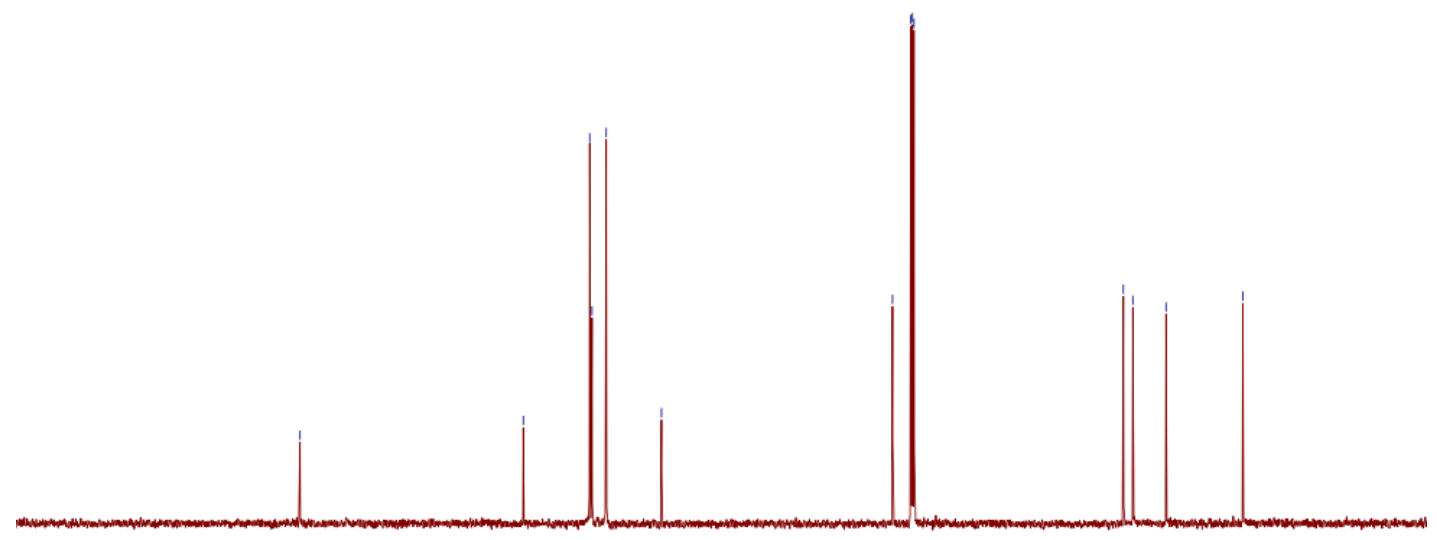

$\begin{array}{lllllllllllllllllllllll}20 & 210 & 200 & 190 & 180 & 170 & 160 & 150 & 140 & 130 & 120 & \begin{array}{l}110 \\ \mathrm{f} 1(\mathrm{ppm})\end{array} & 100 & 90 & 80 & 70 & 60 & 50 & 40 & 30 & 20 & 10 & 0\end{array}$ 
<smiles>C[C@]12OC(=O)C[C@H]1C[C@H](c1ccc(-c3ccccc3)cc1)O2</smiles>

${ }^{1} \mathrm{H}$ NMR $\left(500 \mathrm{MHz}, \mathrm{CDCl}_{3}\right)$

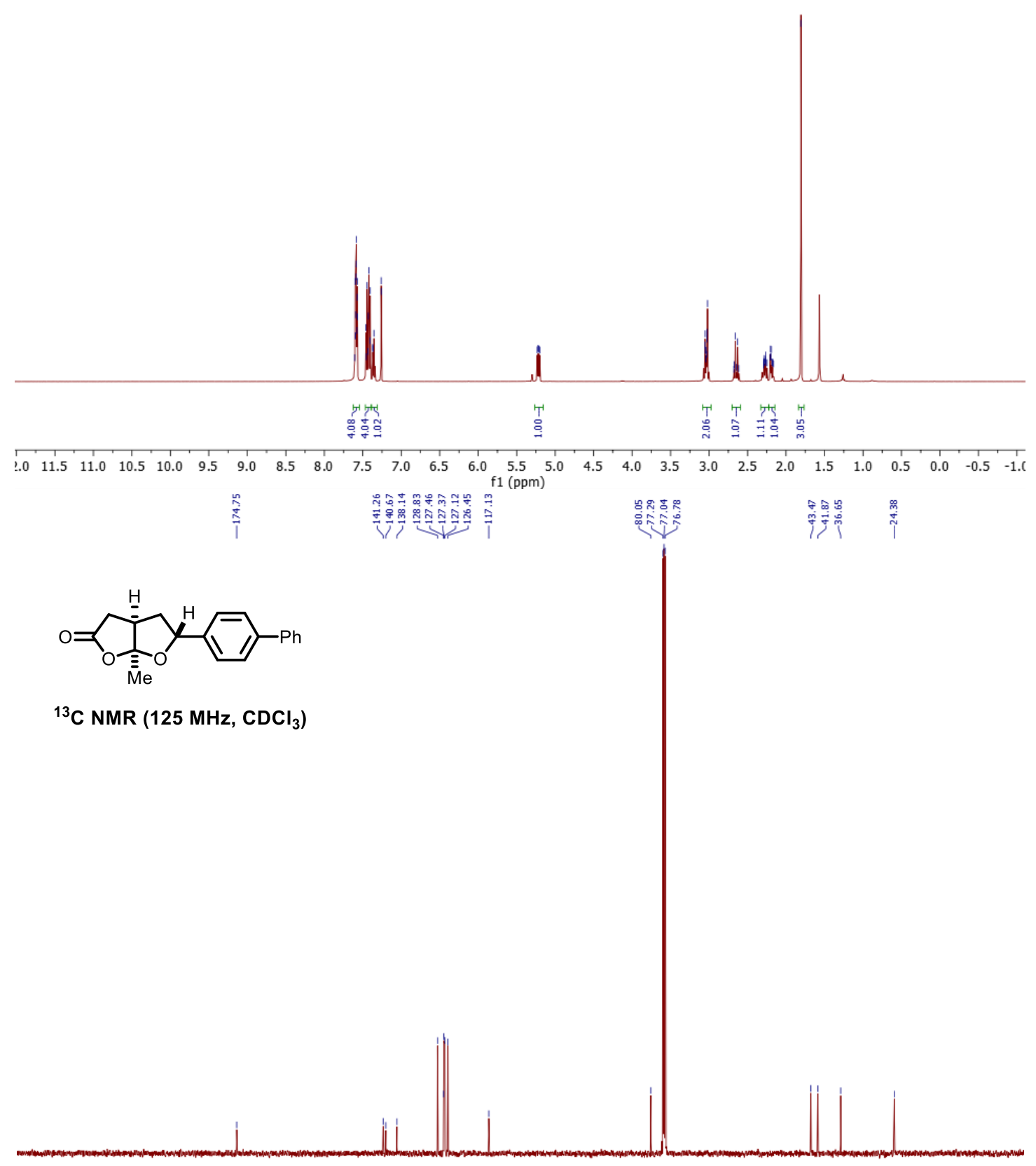

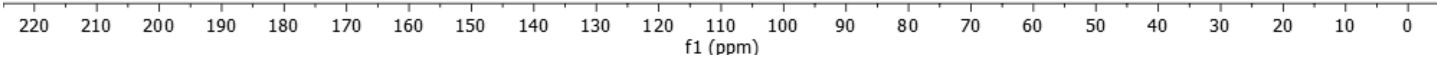



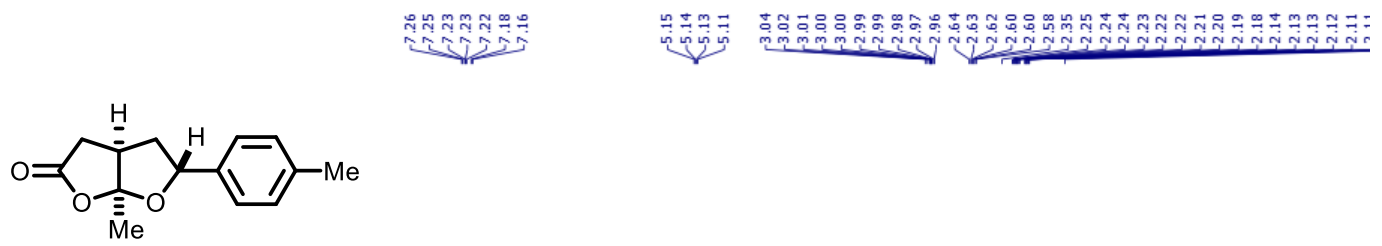

${ }^{1} \mathrm{H}$ NMR (500 MHz, $\mathrm{CDCl}_{3}$ )
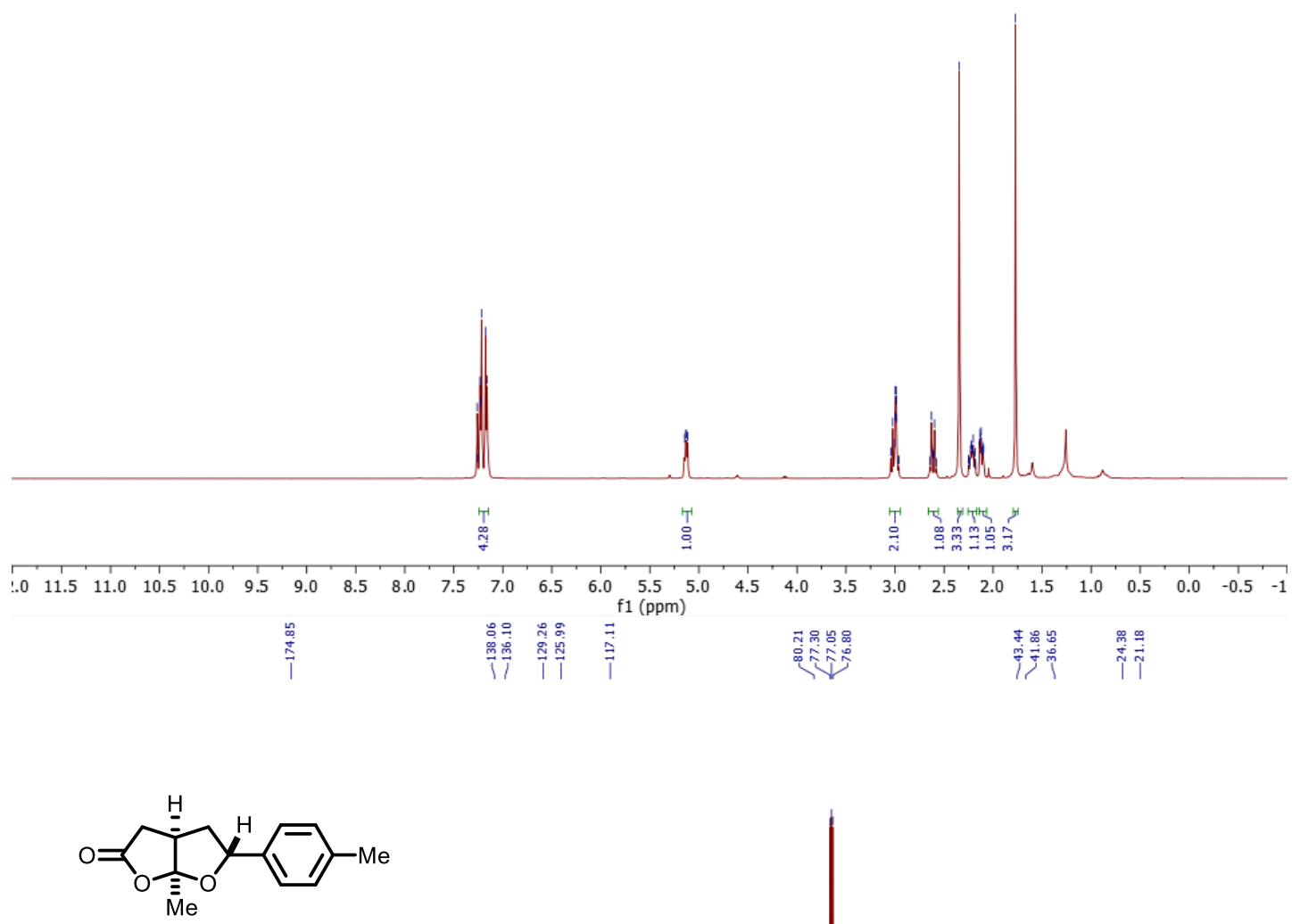

${ }^{13} \mathrm{C}$ NMR $\left(125 \mathrm{MHz}, \mathrm{CDCl}_{3}\right)$

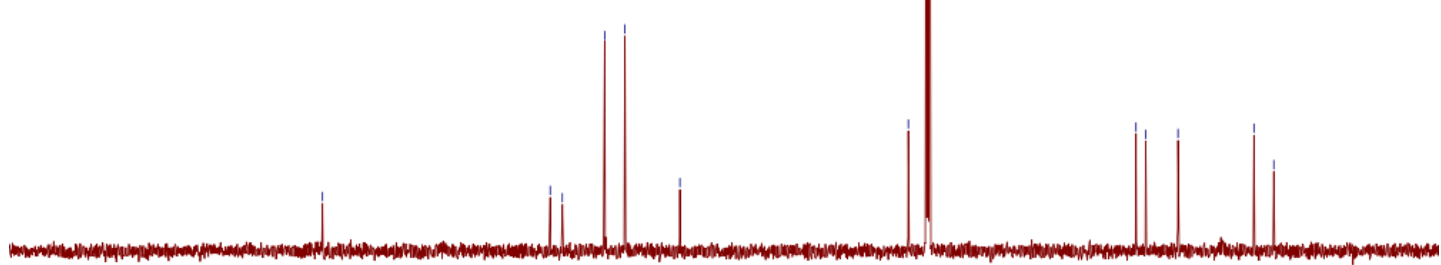

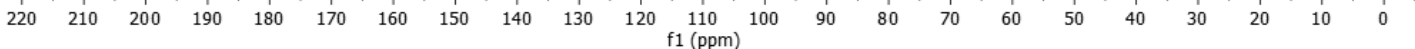




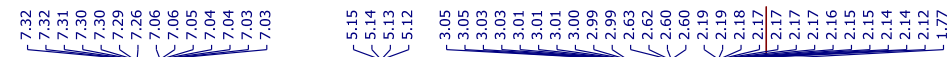

(1)

${ }^{1} \mathrm{H}$ NMR $\left(500 \mathrm{MHz}, \mathrm{CDCl}_{3}\right)$
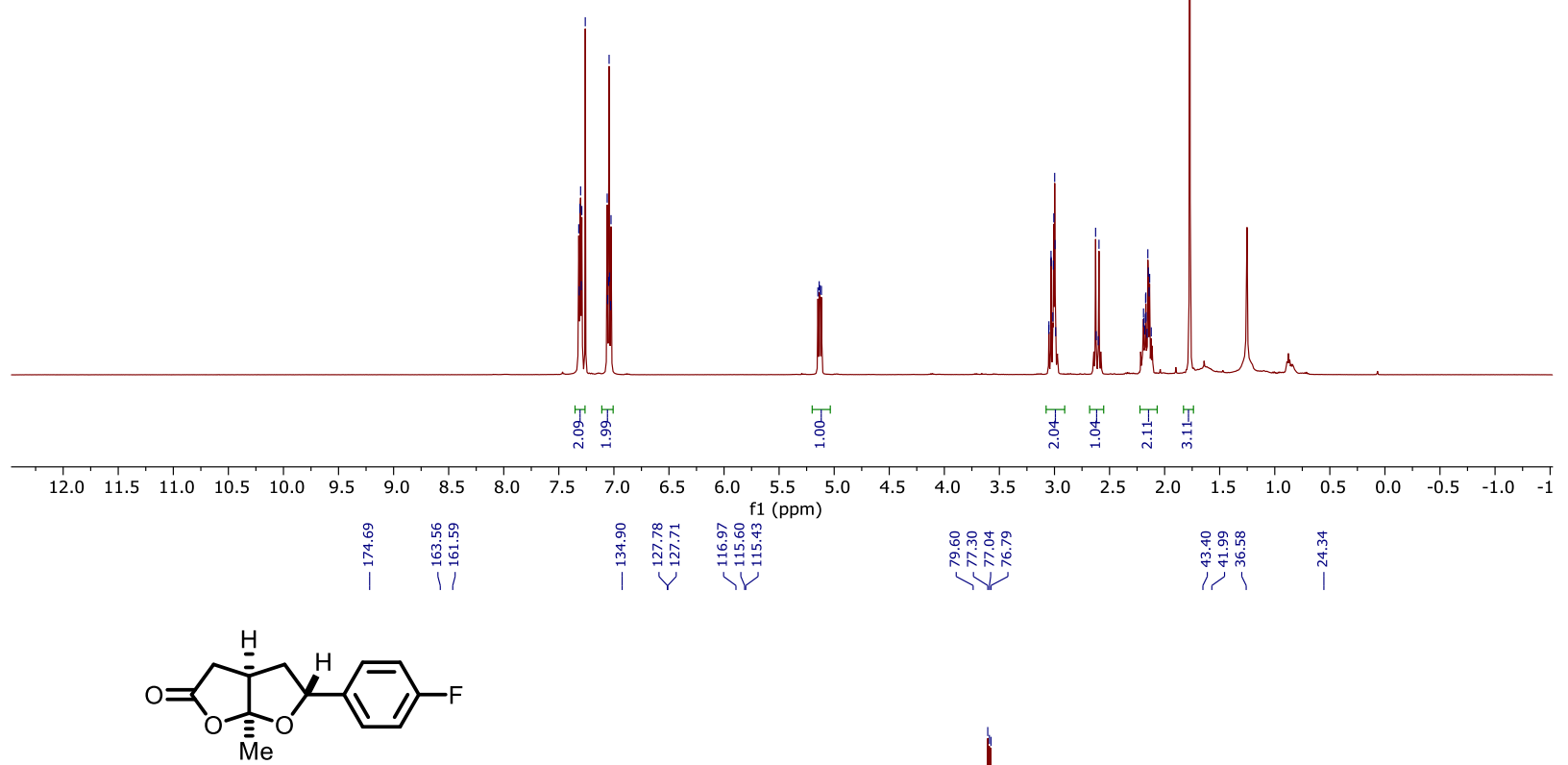

${ }^{13} \mathrm{C}$ NMR $\left(125 \mathrm{MHz}, \mathrm{CDCl}_{3}\right)$
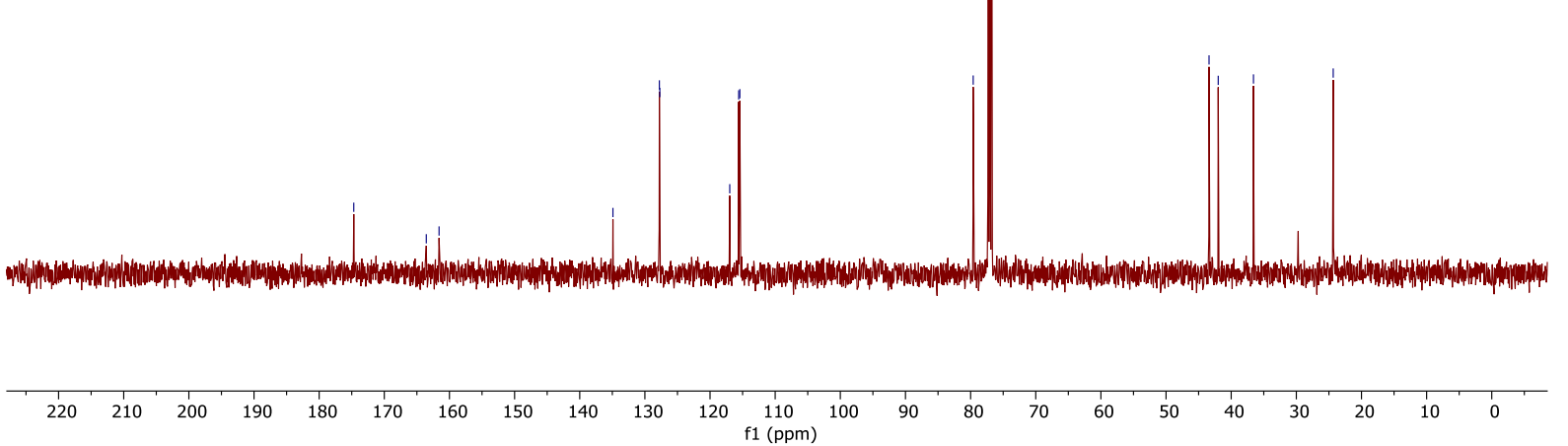


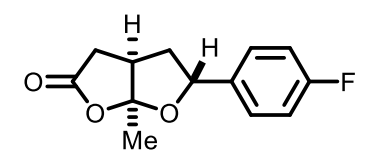

${ }^{19}$ F NMR (471 $\left.\mathrm{MHz}, \mathrm{CDCl}_{3}\right)$

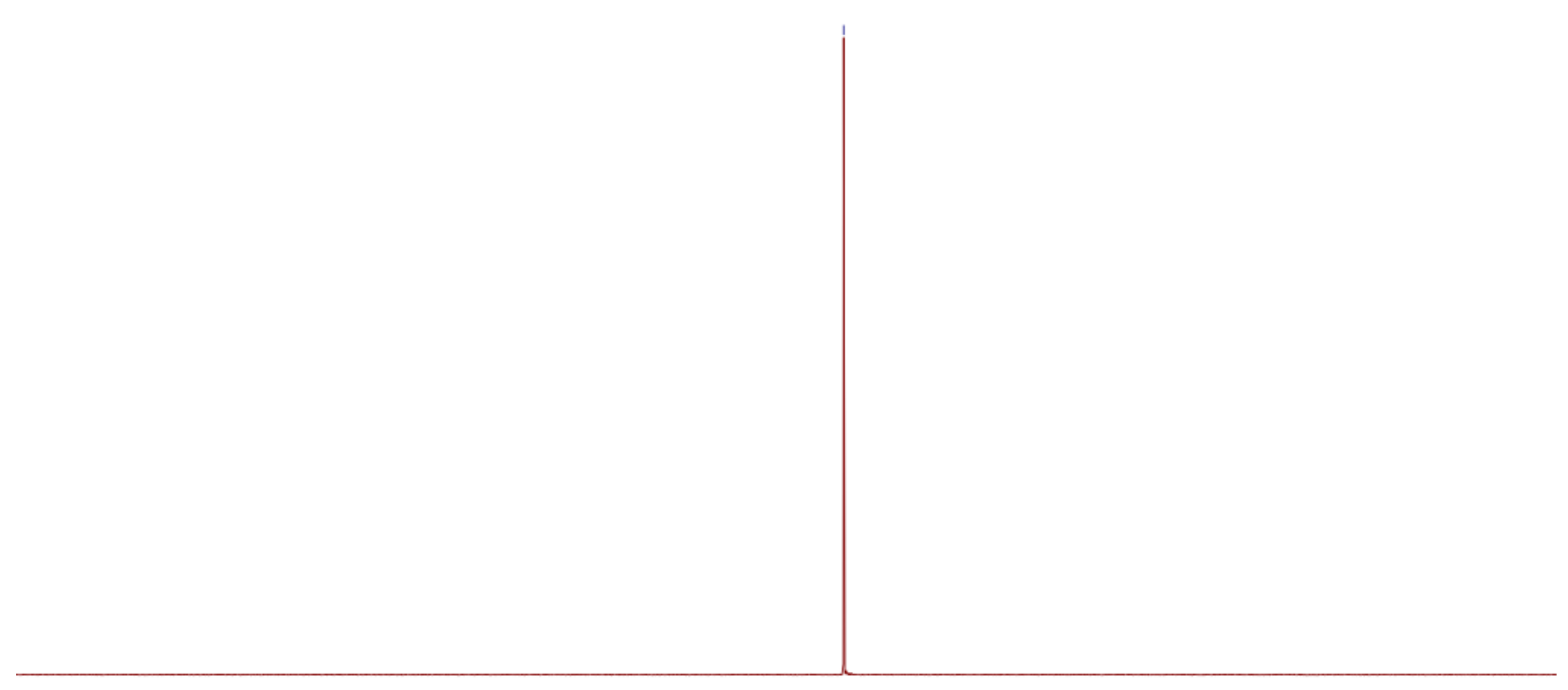

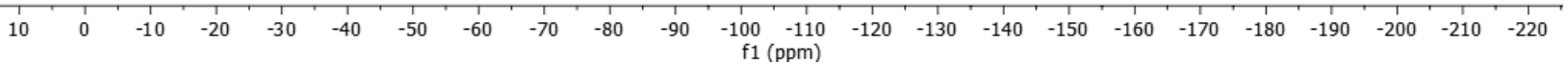



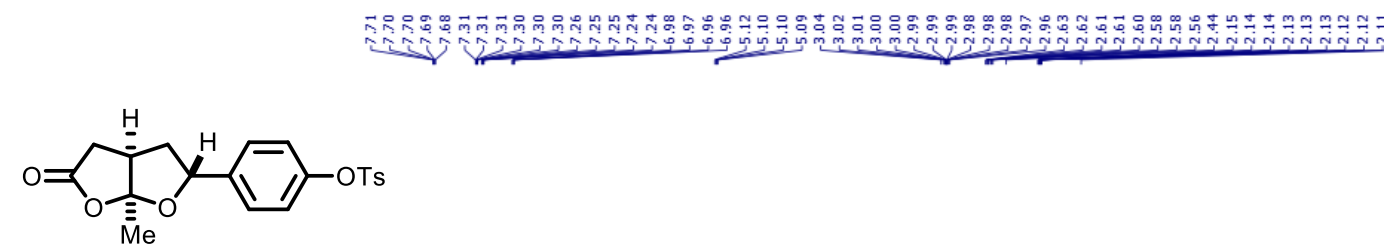

${ }^{1} \mathrm{H}$ NMR (500 MHz, $\left.\mathrm{CDCl}_{3}\right)$

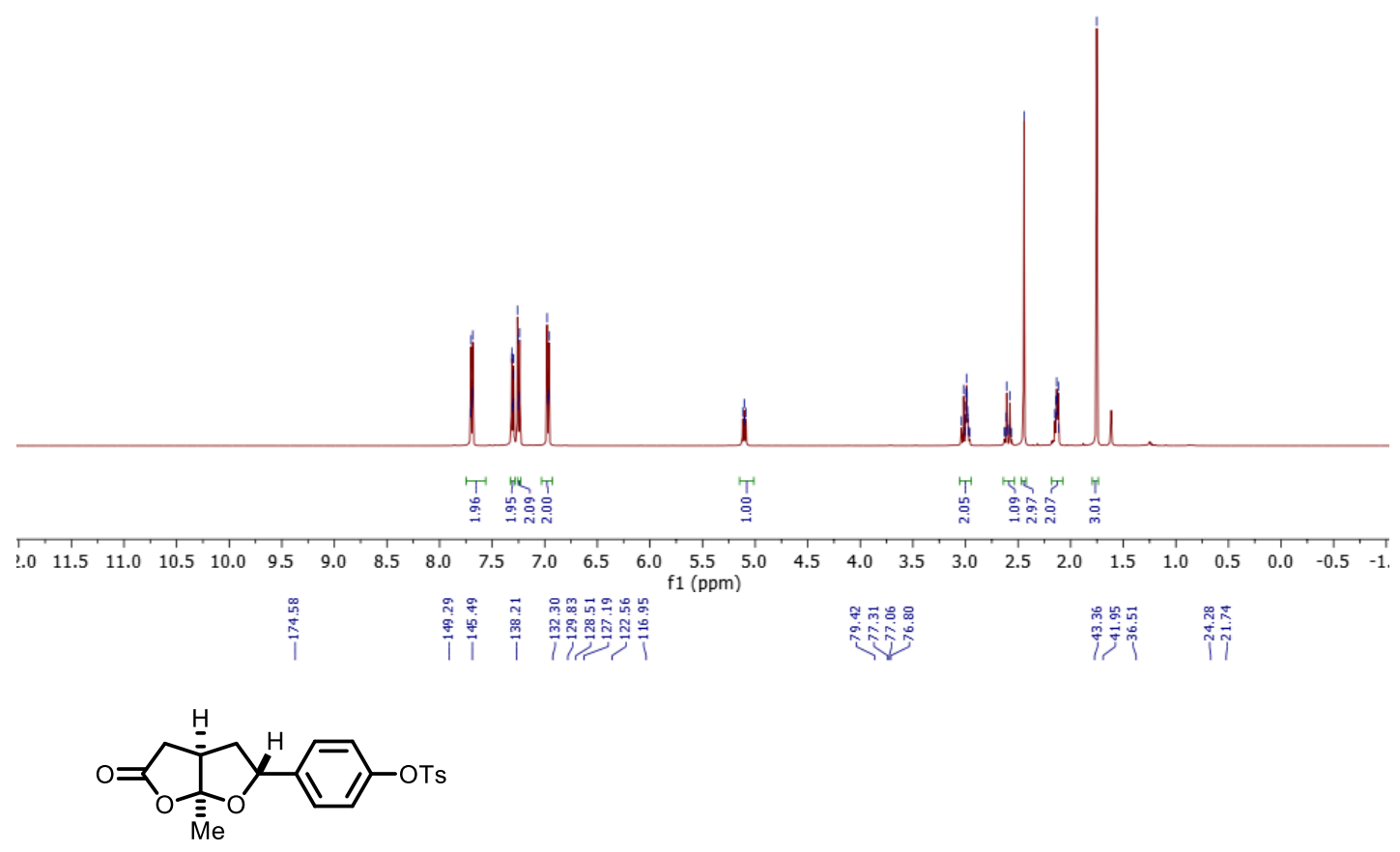

${ }^{13} \mathrm{C}$ NMR $\left(125 \mathrm{MHz}, \mathrm{CDCl}_{3}\right)$

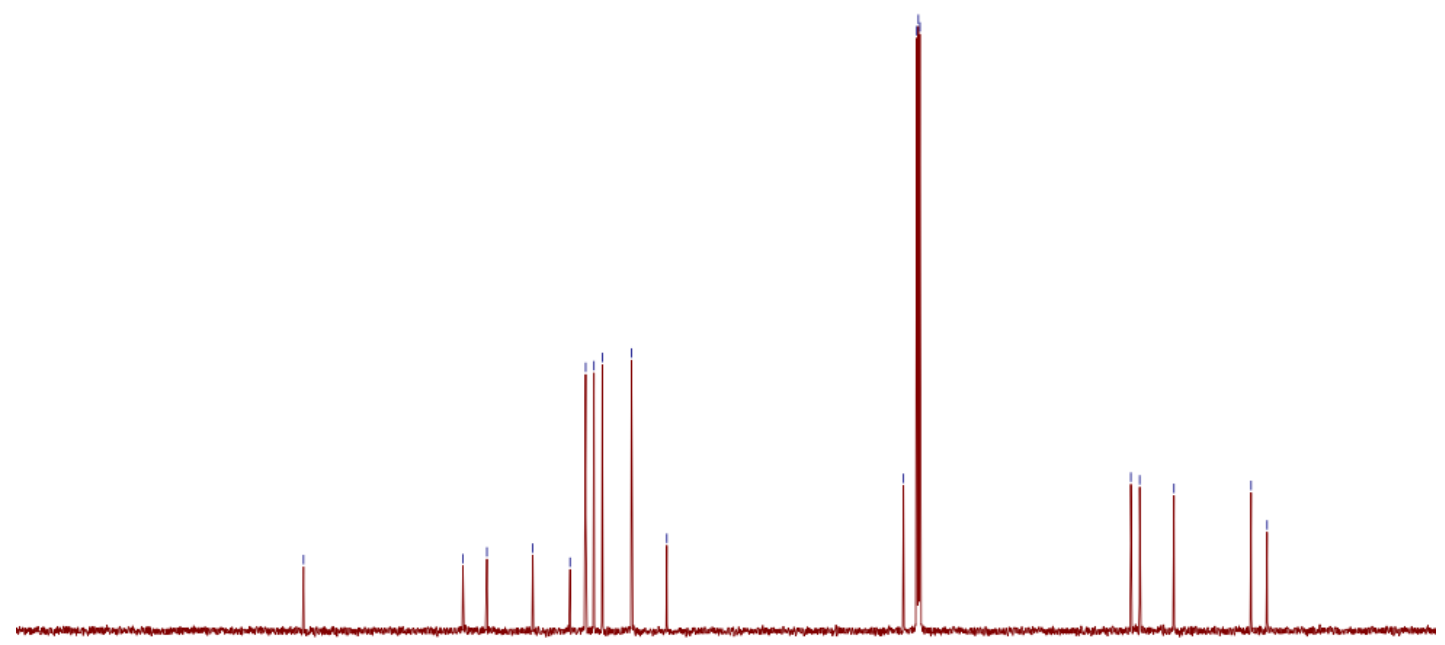

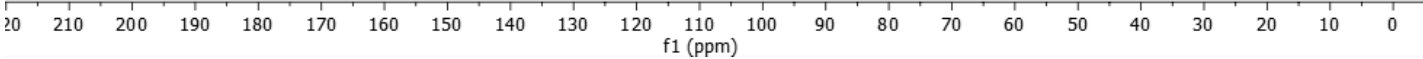




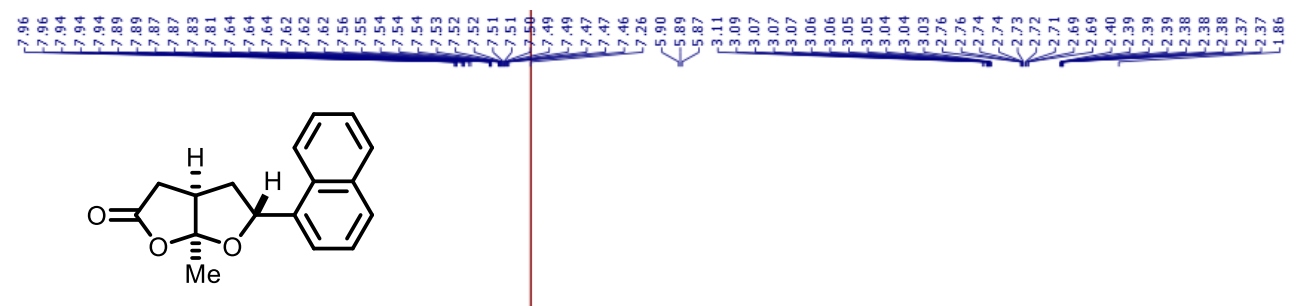

${ }^{1} \mathrm{H}$ NMR (500 MHz, $\mathrm{CDCl}_{3}$ )

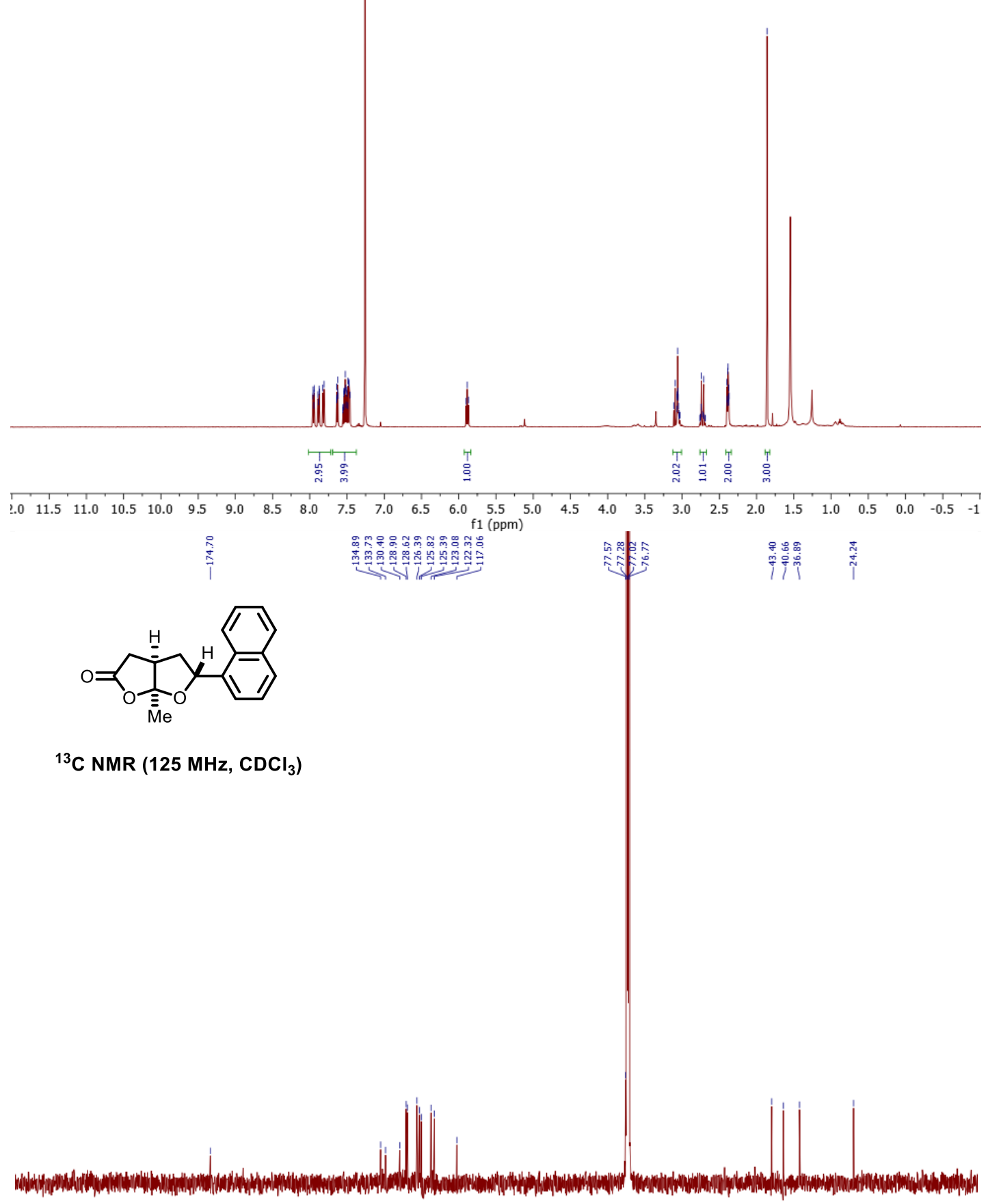

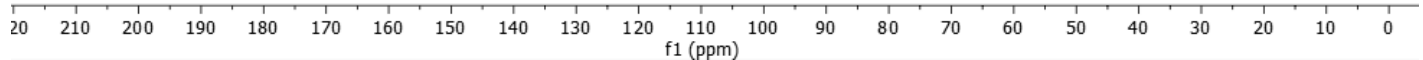




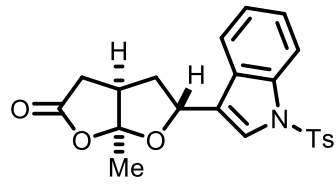

${ }^{1} \mathrm{H}$ NMR $\left(500 \mathrm{MHz}, \mathrm{CDCl}_{3}\right)$

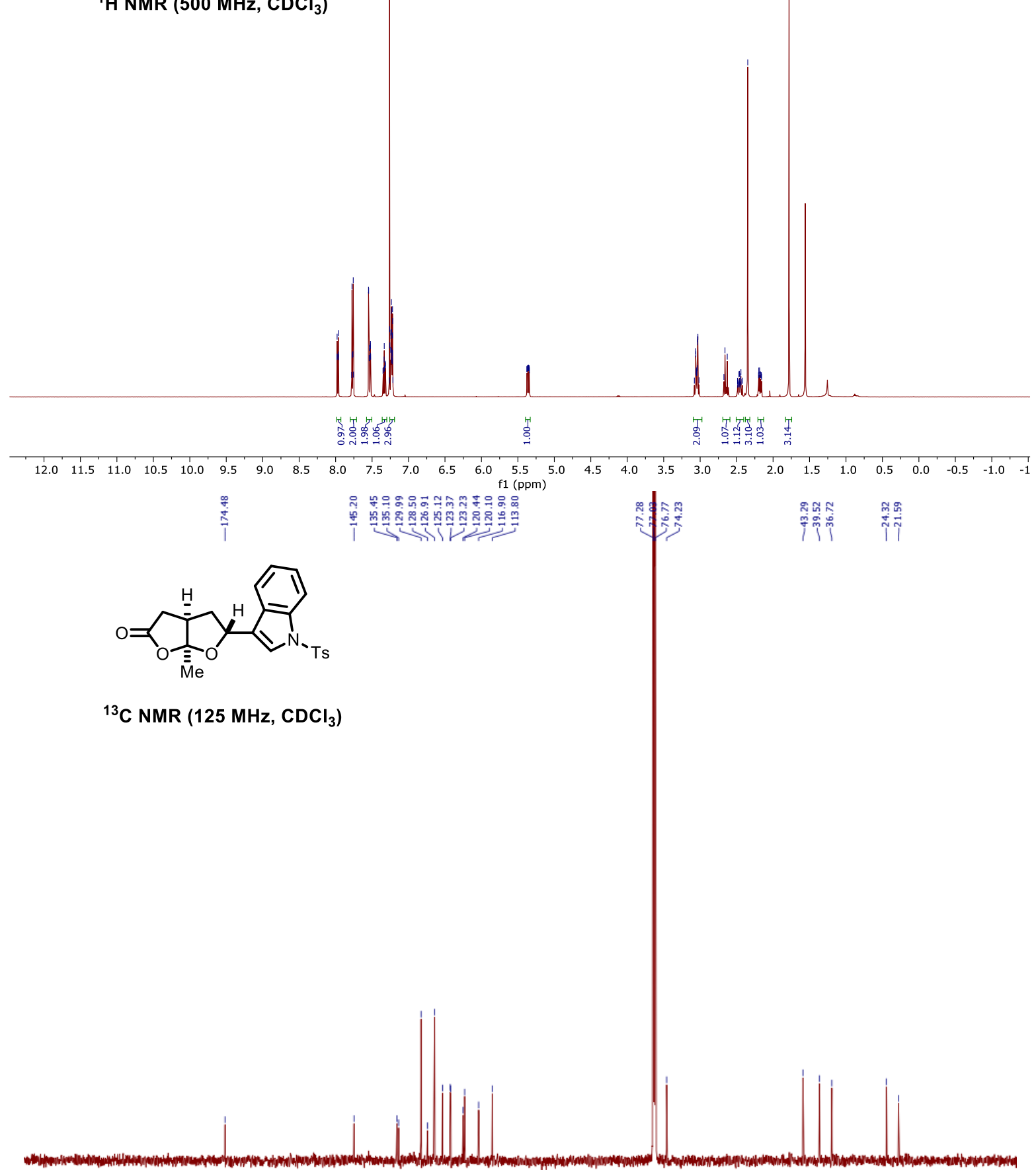

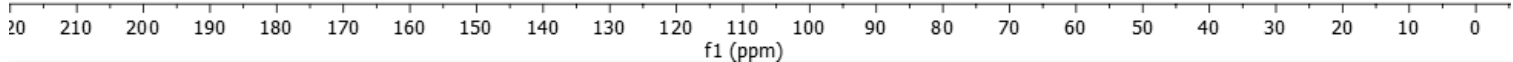


<smiles>C[C@]12OC(=O)C[C@H]1C[C@H](c1ccco1)O2</smiles>

${ }^{1} \mathrm{H}$ NMR $\left(500 \mathrm{MHz}, \mathrm{CDCl}_{3}\right)$

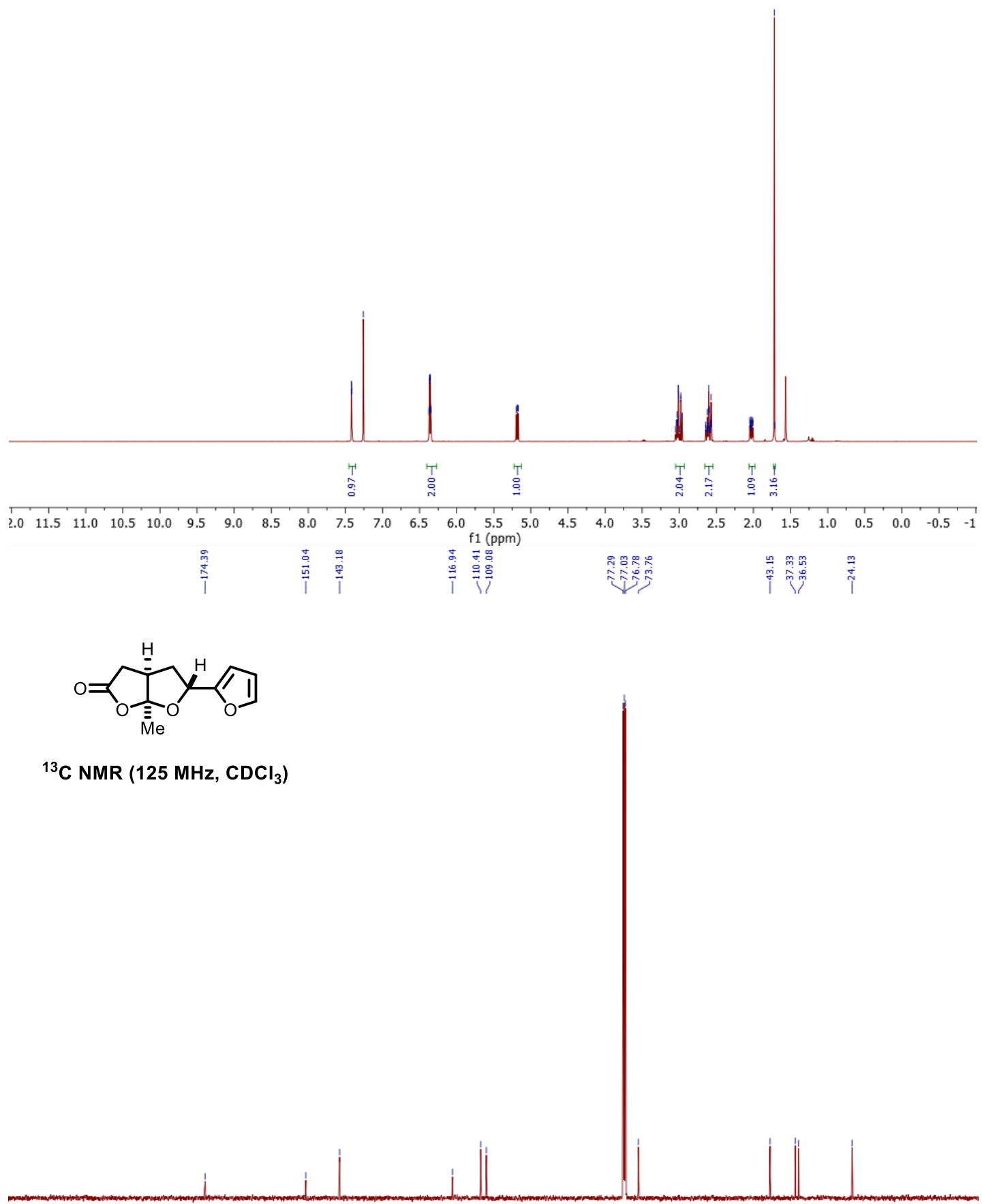

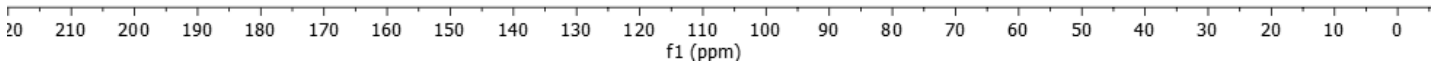


<smiles>C[C@]12OC(=O)C[C@H]1C[C@H](c1cccs1)O2</smiles>

${ }^{1} \mathrm{H}$ NMR $\left(500 \mathrm{MHz}, \mathrm{CDCl}_{3}\right)$

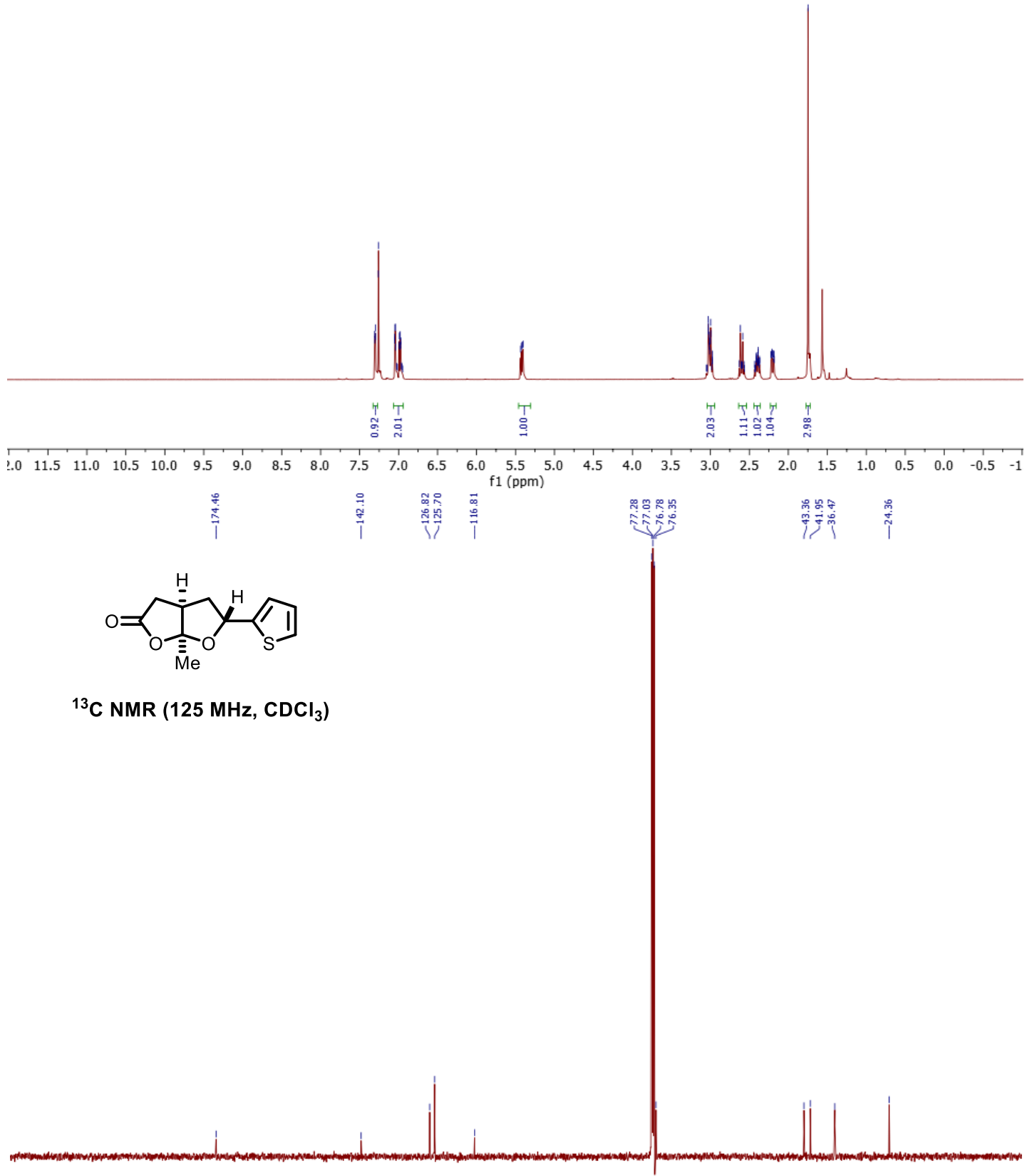

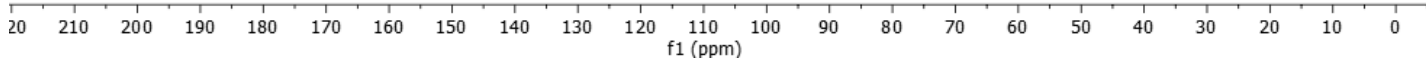




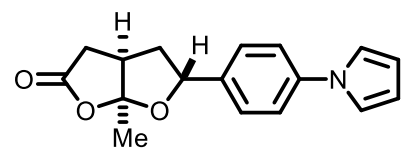

${ }^{1} \mathrm{H}$ NMR (500 MHz, $\left.\mathrm{CDCl}_{3}\right)$
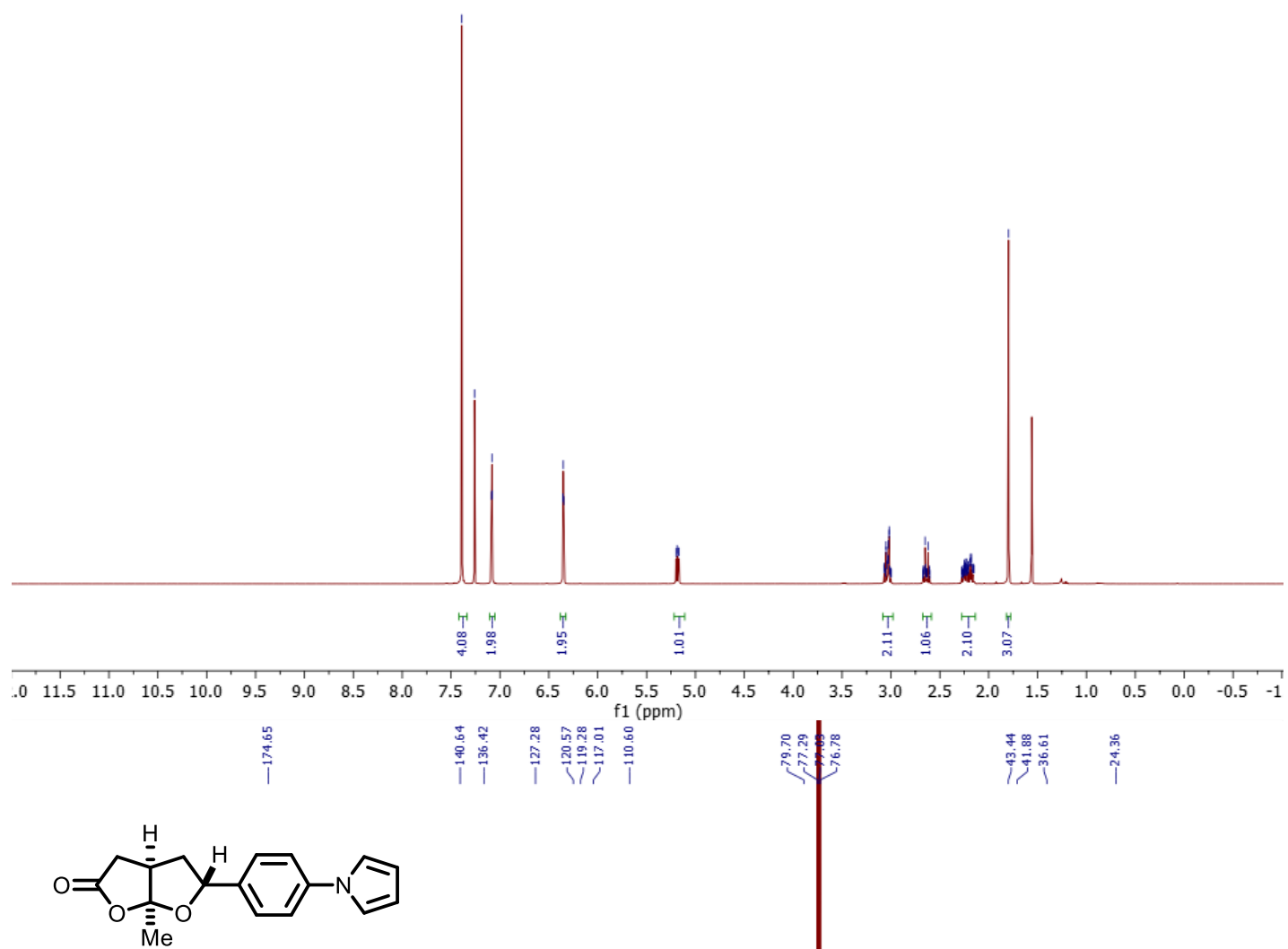

${ }^{13} \mathrm{C}$ NMR $\left(125 \mathrm{MHz}, \mathrm{CDCl}_{3}\right)$

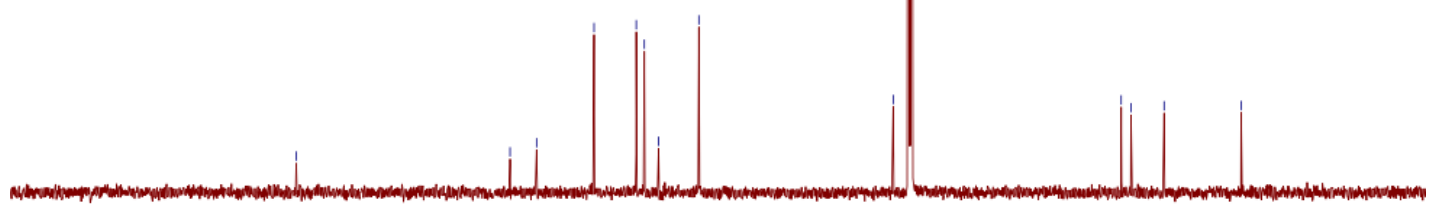

$\begin{array}{lllllllllllllllllllllll}20 & 210 & 200 & 190 & 180 & 170 & 160 & 150 & 140 & 130 & 120 & \begin{array}{c}110 \\ \mathrm{f} 1(\mathrm{ppm})\end{array} & 100 & 90 & 80 & 70 & 60 & 50 & 40 & 30 & 20 & 10 & 0\end{array}$ 


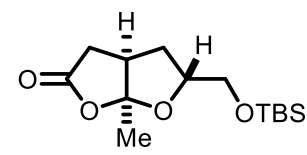

${ }^{1} \mathrm{H}$ NMR (500 MHz, $\left.\mathrm{CDCl}_{3}\right)$

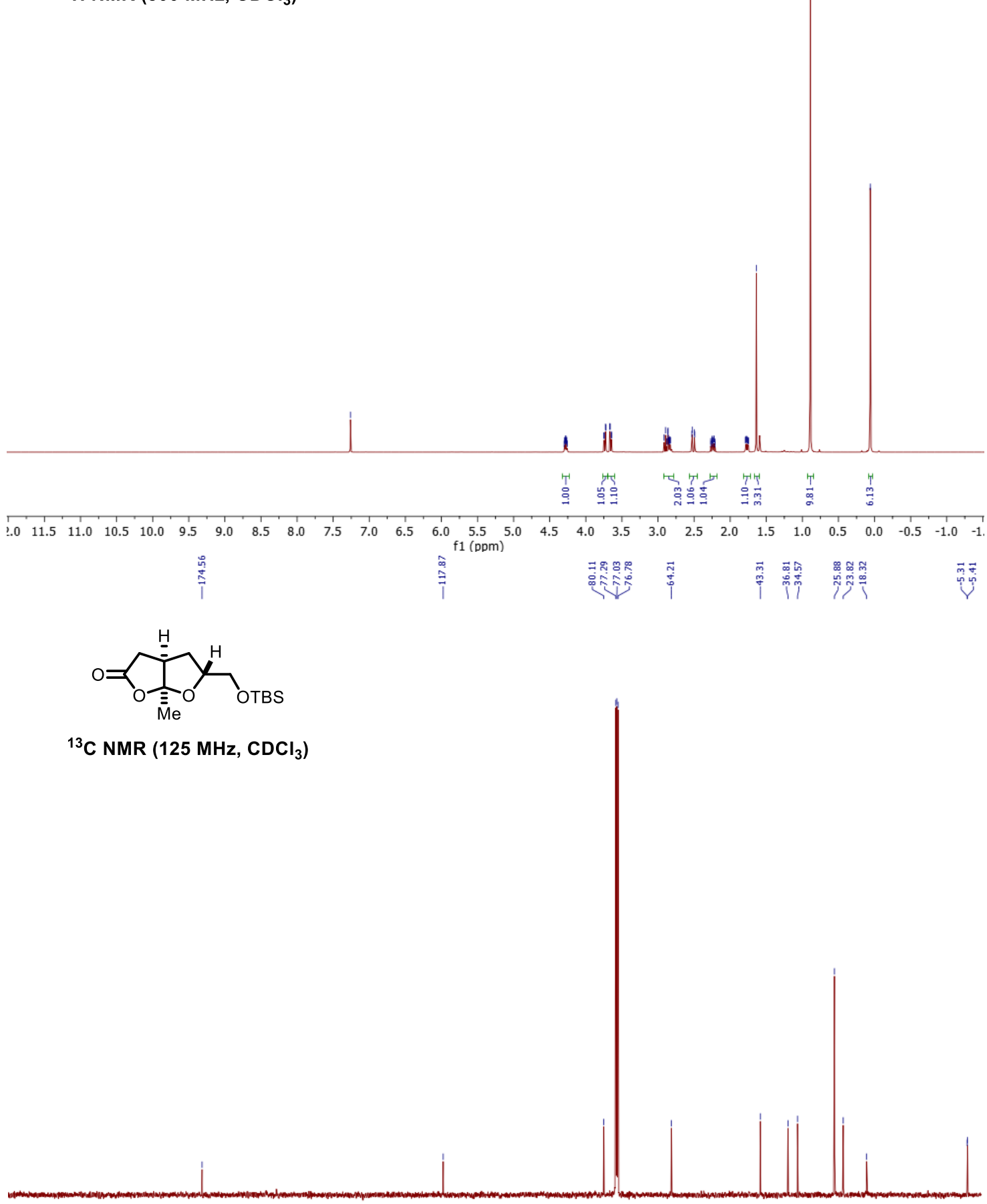

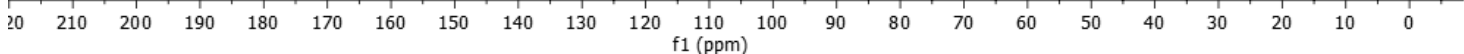




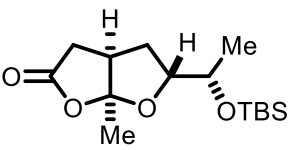

${ }^{1} \mathrm{H}$ NMR $\left(500 \mathrm{MHz}, \mathrm{CDCl}_{3}\right)$

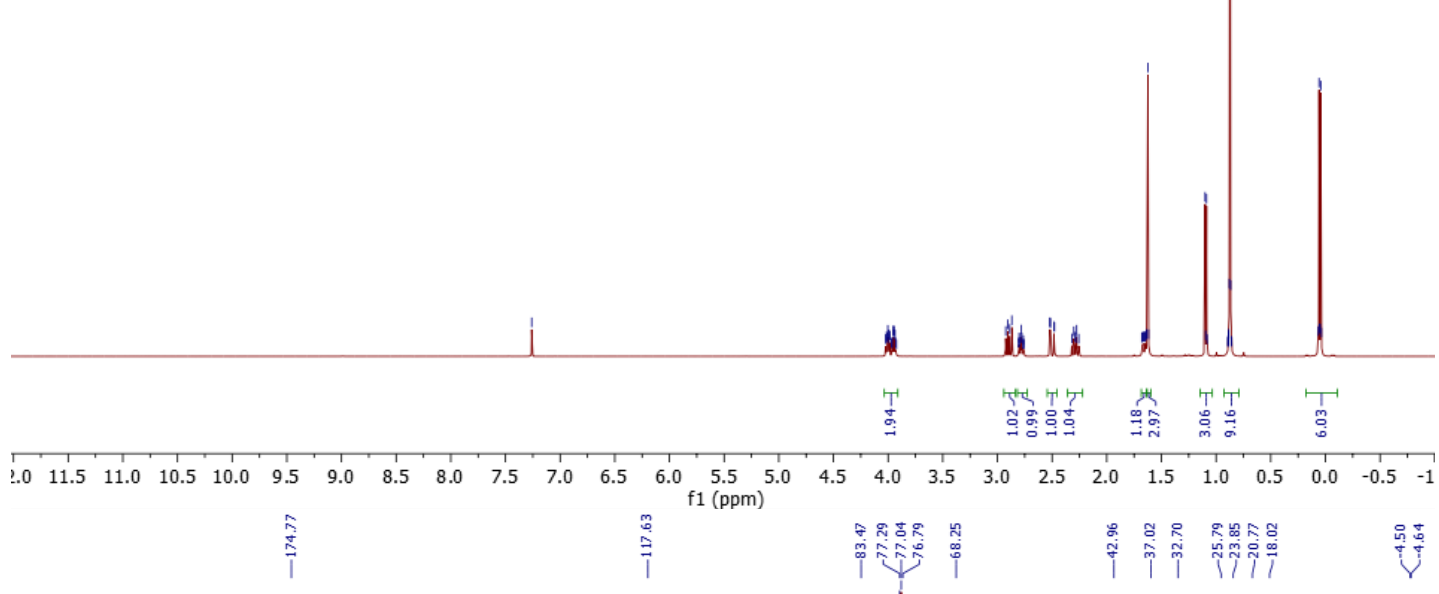

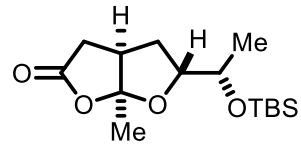

${ }^{13} \mathrm{C}$ NMR $\left(125 \mathrm{MHz}, \mathrm{CDCl}_{3}\right)$

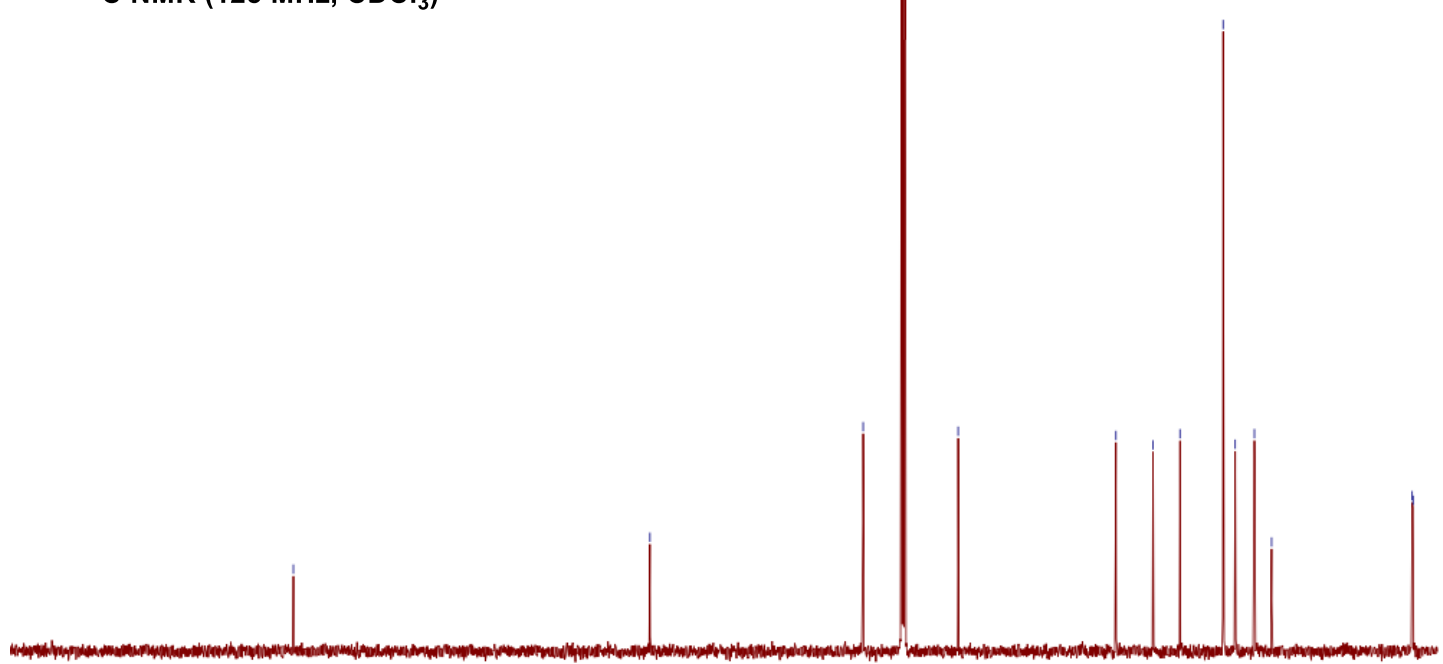

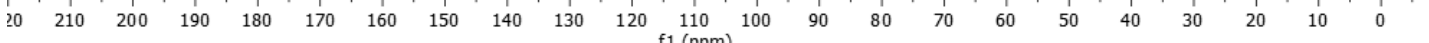




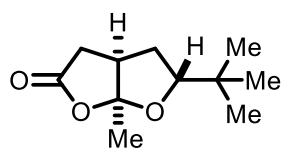

${ }^{1} \mathrm{H}$ NMR $\left(500 \mathrm{MHz}, \mathrm{CDCl}_{3}\right)$
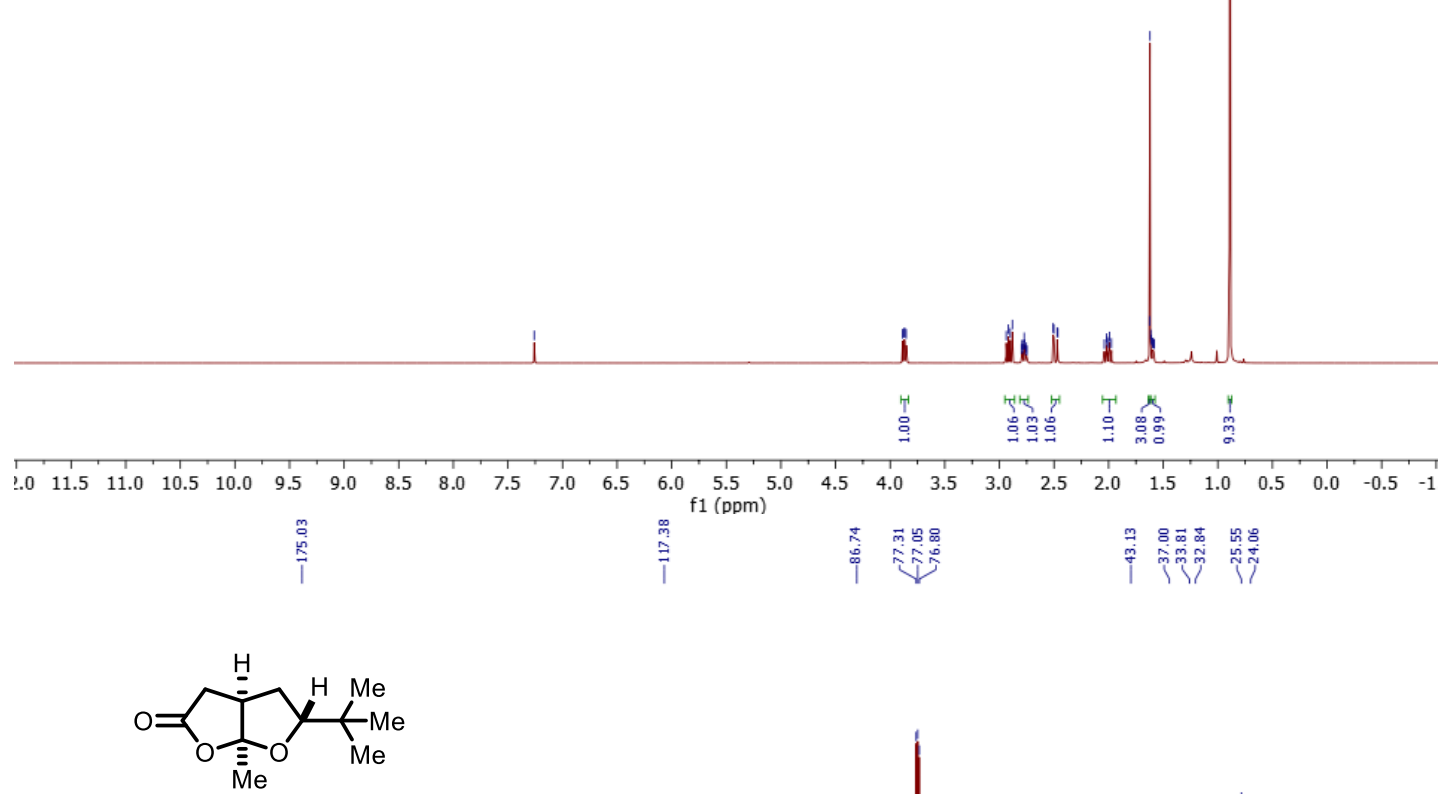

${ }^{13} \mathrm{C}$ NMR (125 $\left.\mathrm{MHz}, \mathrm{CDCl}_{3}\right)$

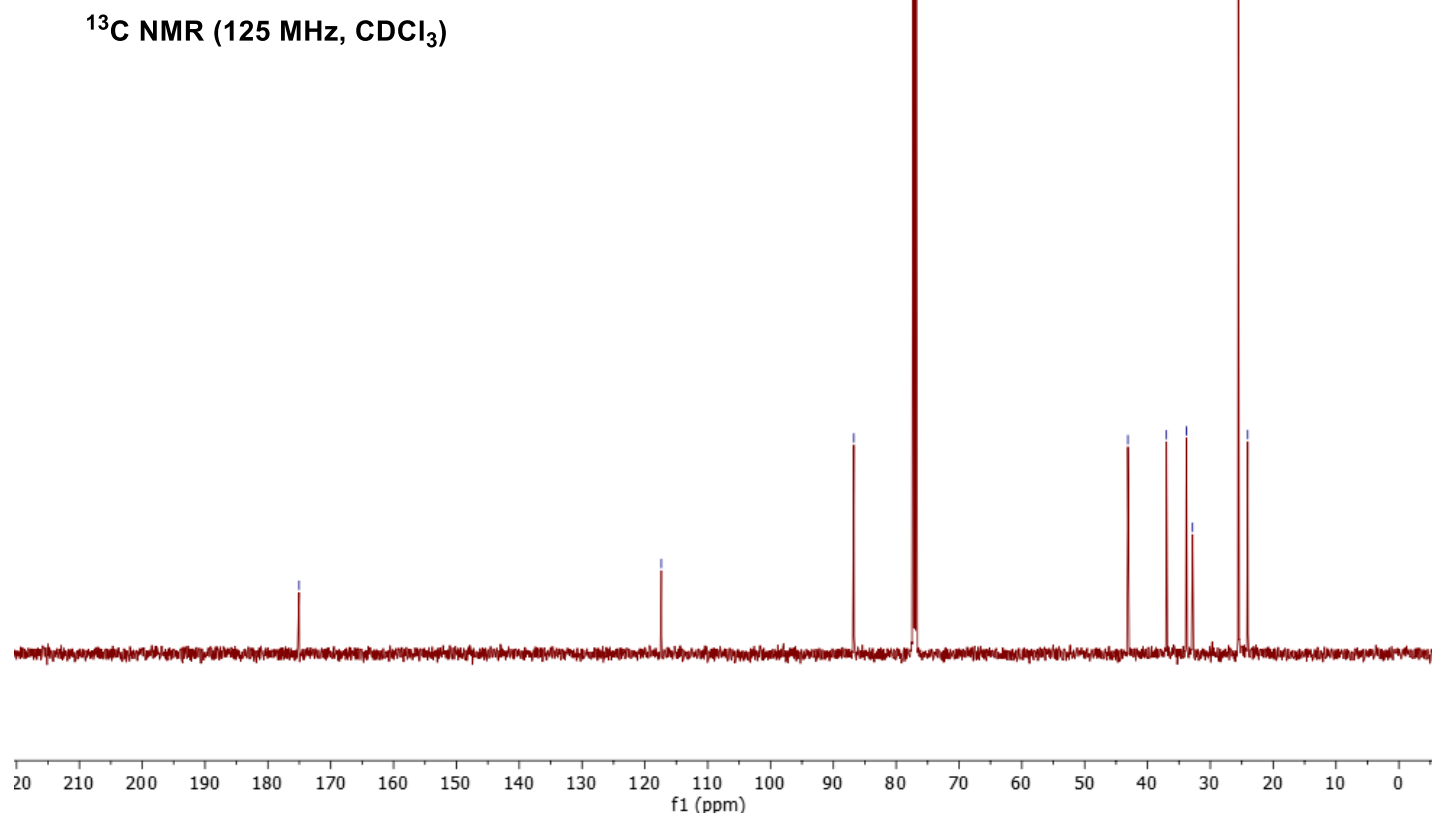




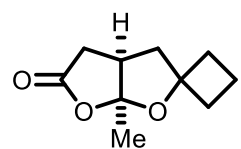

${ }^{1} \mathrm{H}$ NMR (500 MHz, $\mathrm{CDCl}_{3}$ )

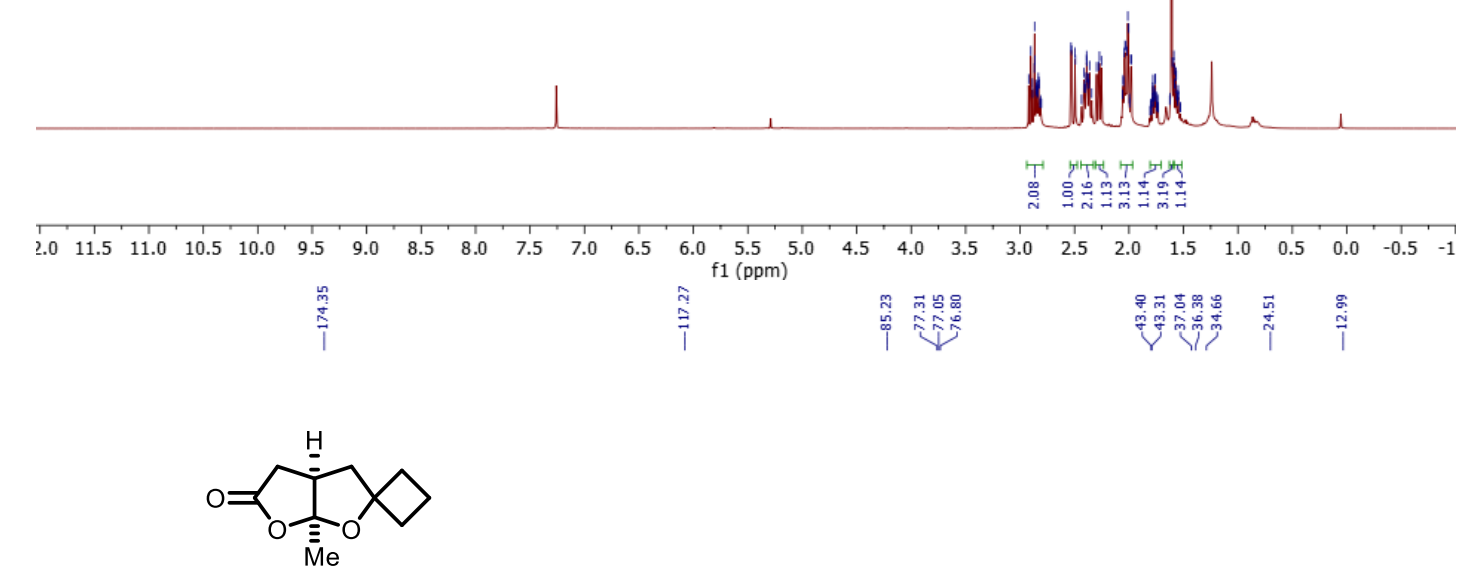

${ }^{13} \mathrm{C}$ NMR (125 MHz, $\mathrm{CDCl}_{3}$ )

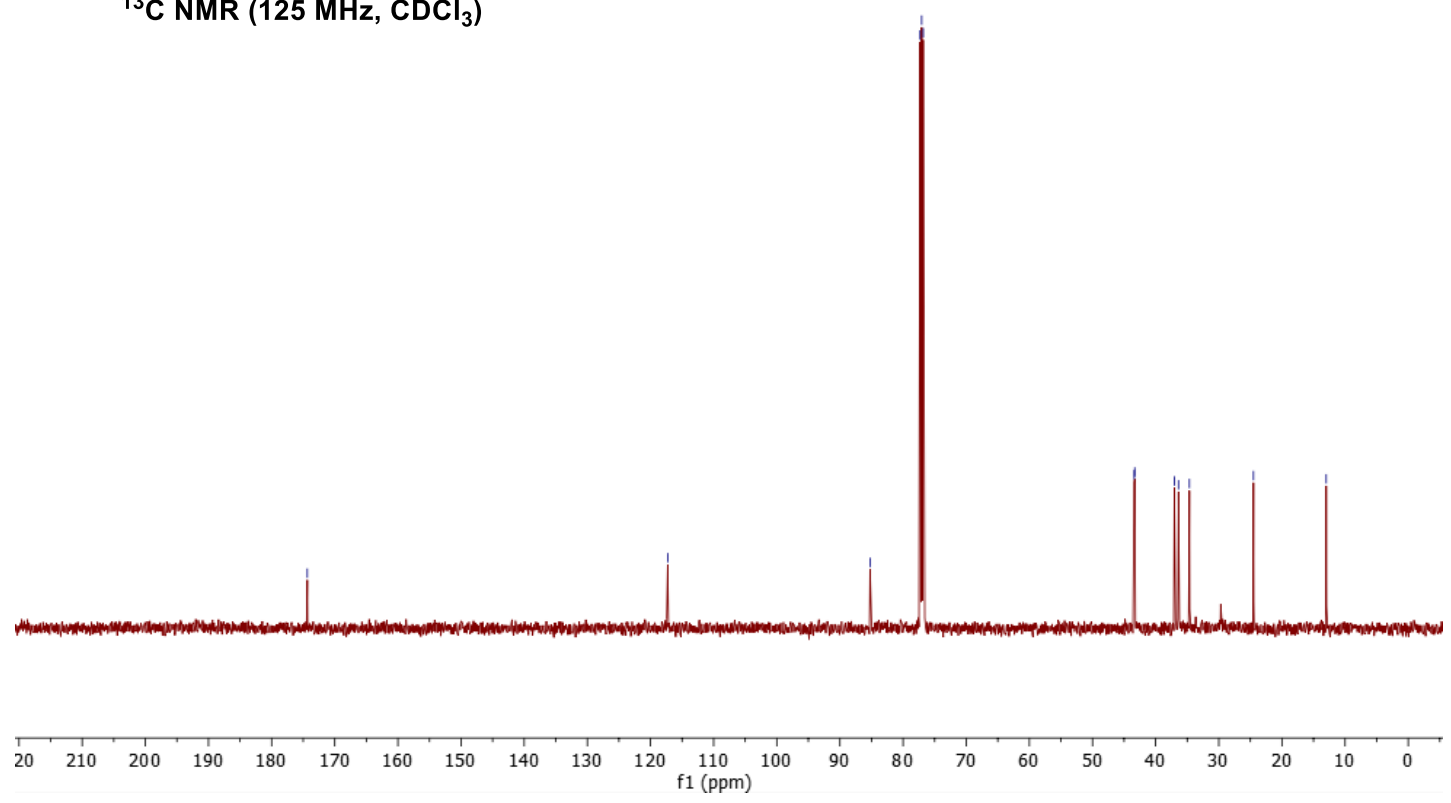




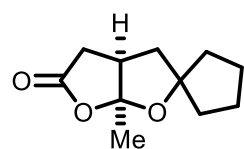

${ }^{1} \mathrm{H}$ NMR $\left(500 \mathrm{MHz}, \mathrm{CDCl}_{3}\right)$

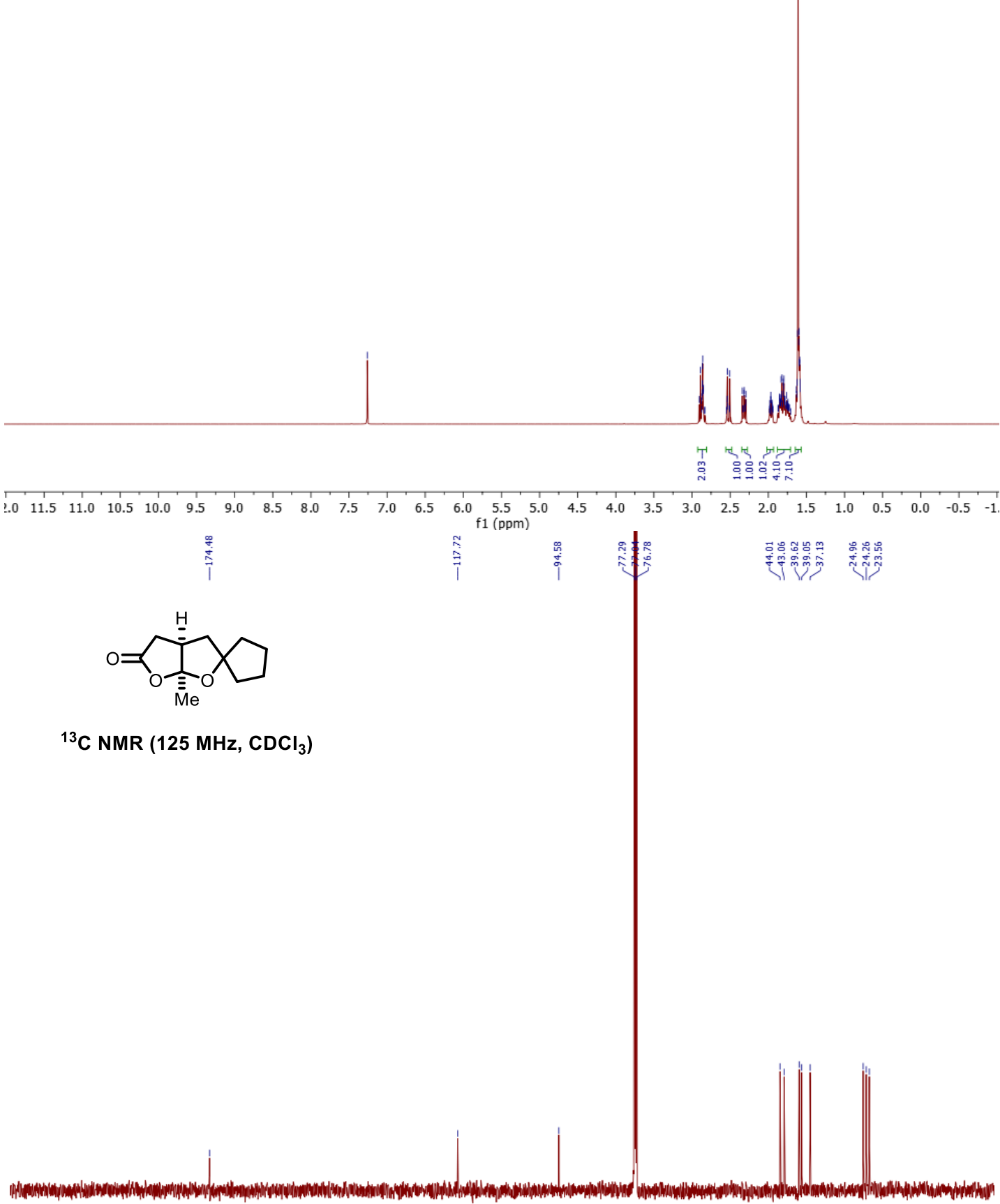

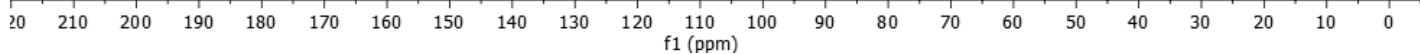




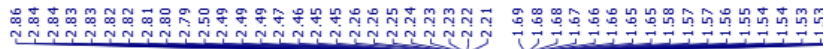

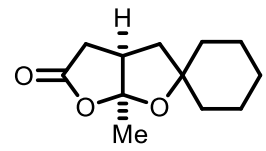

${ }^{1} \mathrm{H}$ NMR (500 MHz, $\left.\mathrm{CDCl}_{3}\right)$

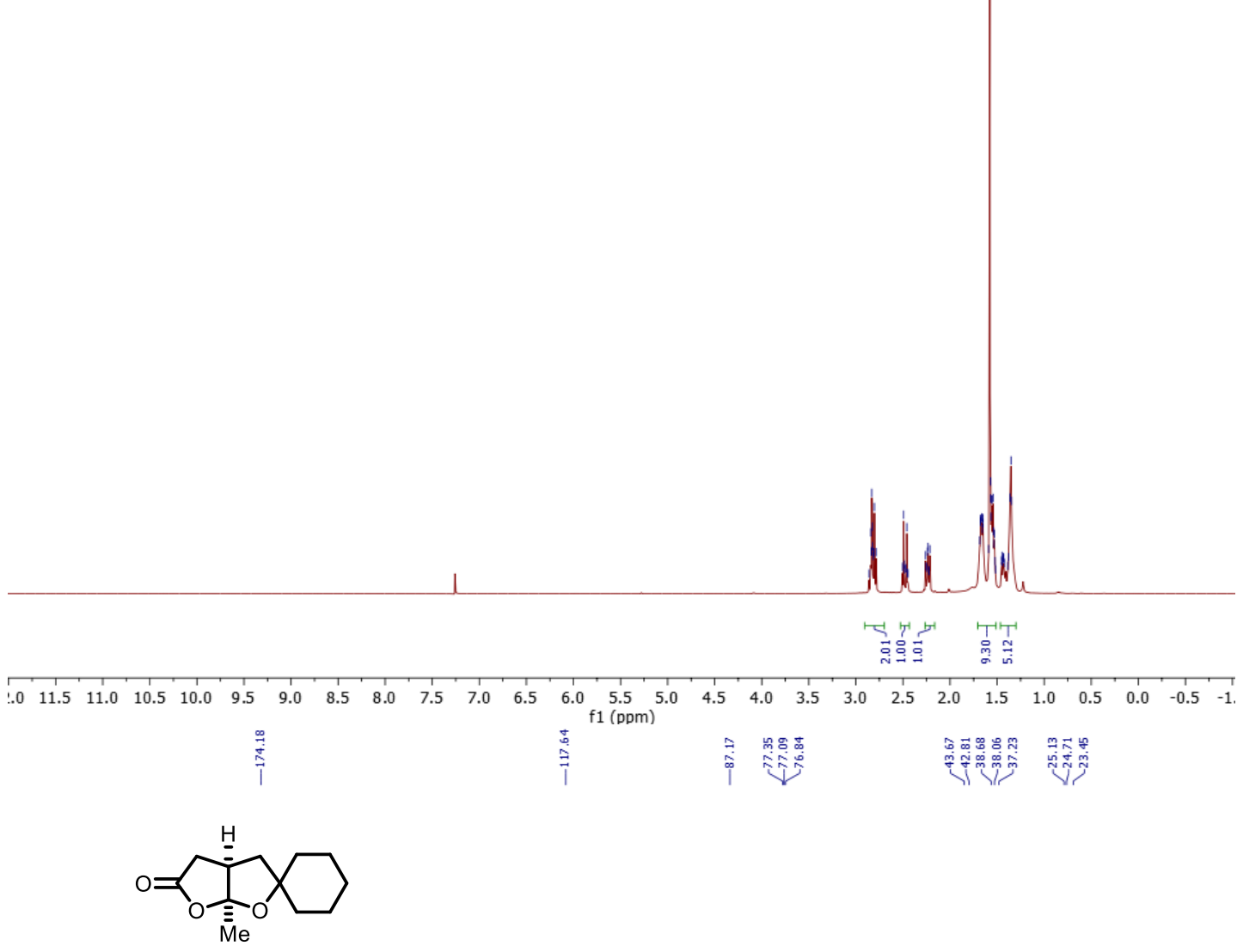

${ }^{13} \mathrm{C}$ NMR $\left(125 \mathrm{MHz}, \mathrm{CDCl}_{3}\right)$

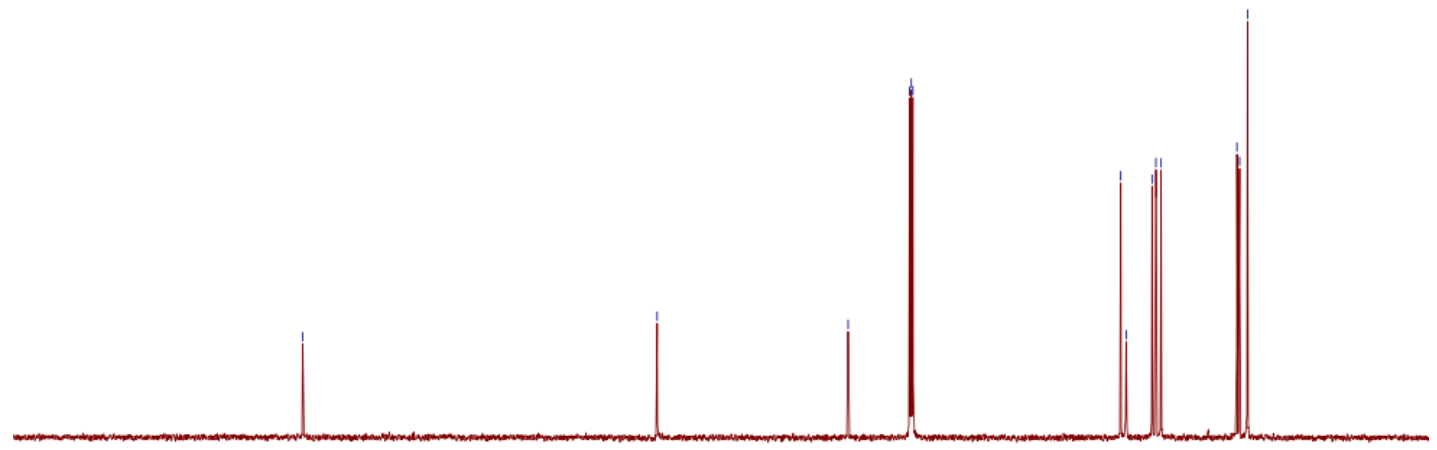

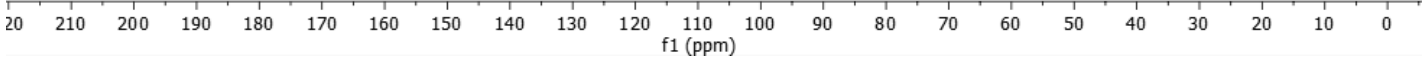




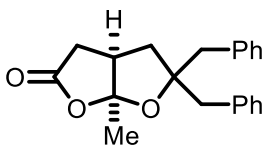

${ }^{1} \mathrm{H} \mathrm{NMR}\left(500 \mathrm{MHz}, \mathrm{CDCl}_{3}\right)$

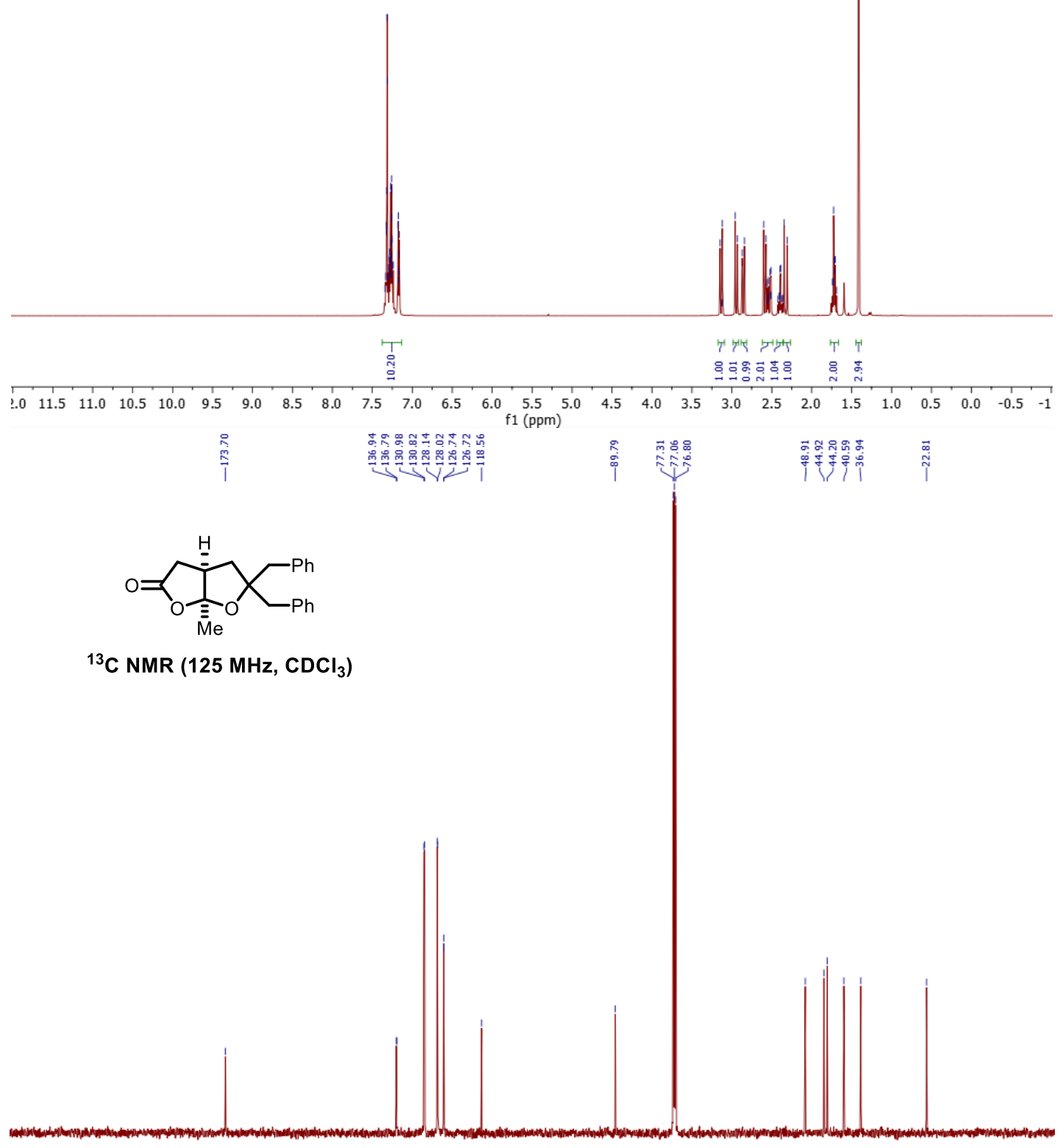

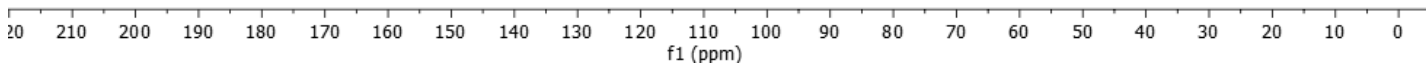


<smiles>CCCCC[C@]12OC(=O)C[C@H]1CC(c1ccccc1)O2</smiles>

${ }^{1} \mathrm{H}$ NMR (500 MHz, $\mathrm{CDCl}_{3}$ )

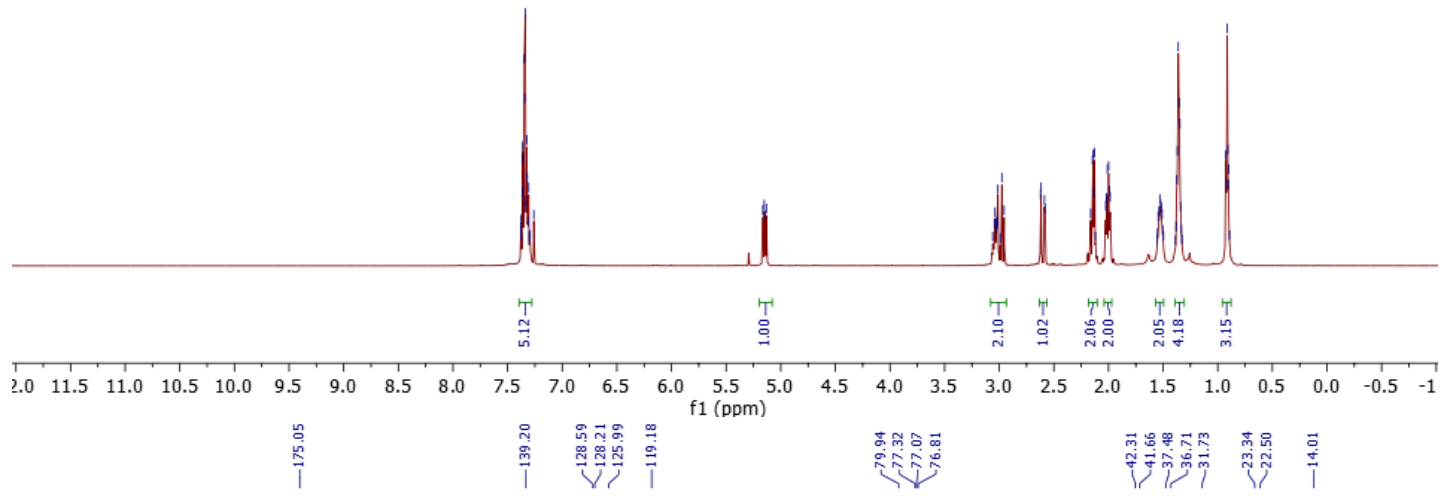<smiles>CCCCC[C@]12OC(=O)C[C@H]1CC(c1ccccc1)O2</smiles>

${ }^{13} \mathrm{C}$ NMR (125 MHz, $\mathrm{CDCl}_{3}$ )

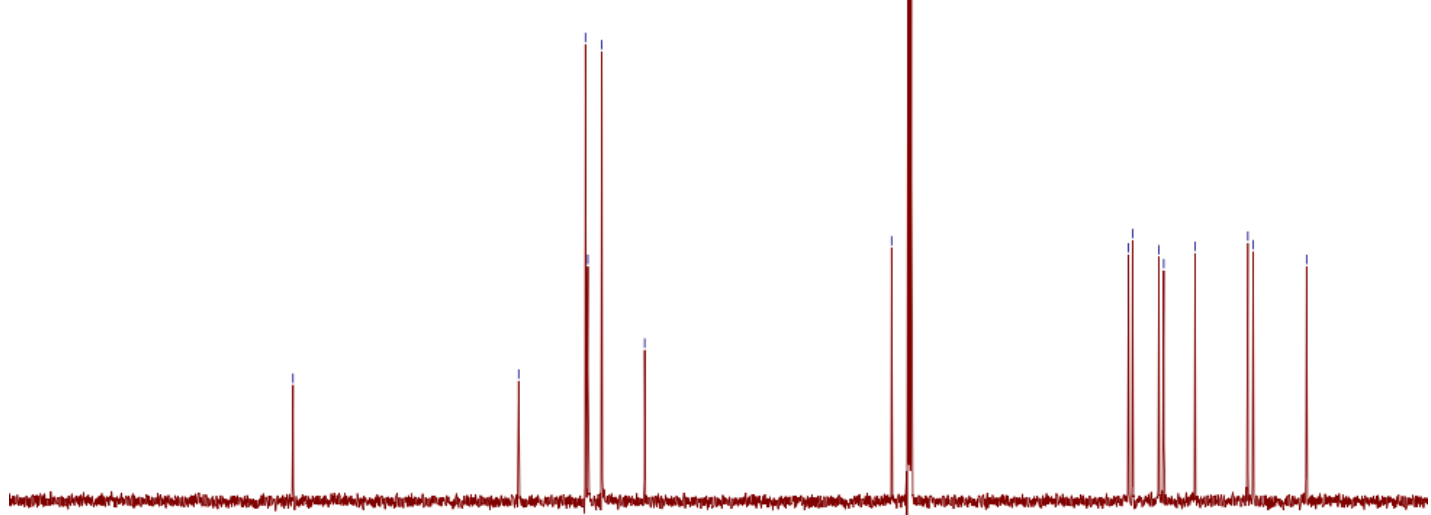

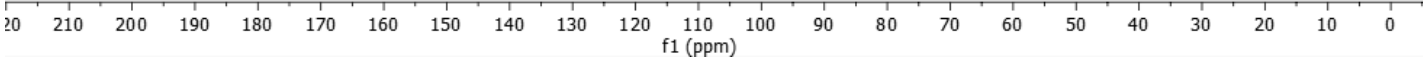




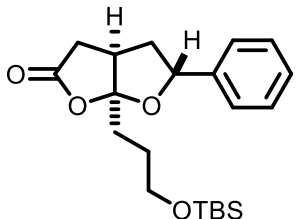

${ }^{1} \mathrm{H}$ NMR $\left(500 \mathrm{MHz}, \mathrm{CDCl}_{3}\right)$

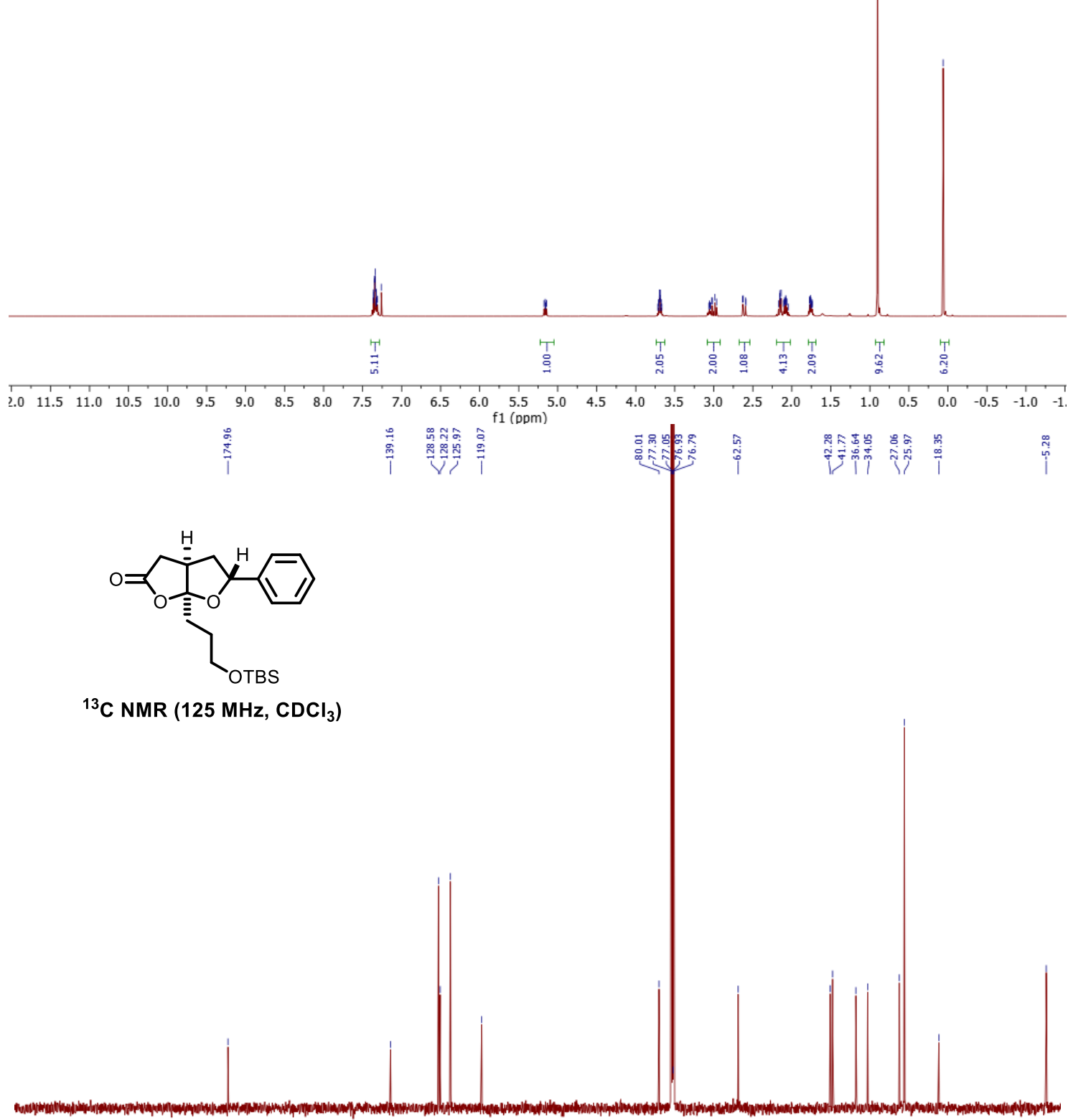

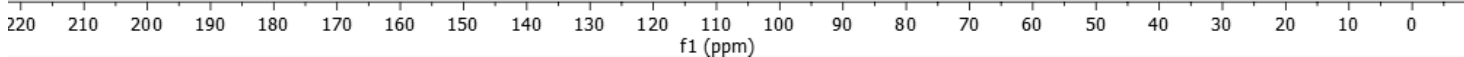


<smiles>O=C1C[C@H]2CC(c3ccccc3)(O2)OC1(C1CCCCC1)C1CCCCC1</smiles>

${ }^{1} \mathrm{H}$ NMR (500 $\left.\mathrm{MHz}, \mathrm{CDCl}_{3}\right)$

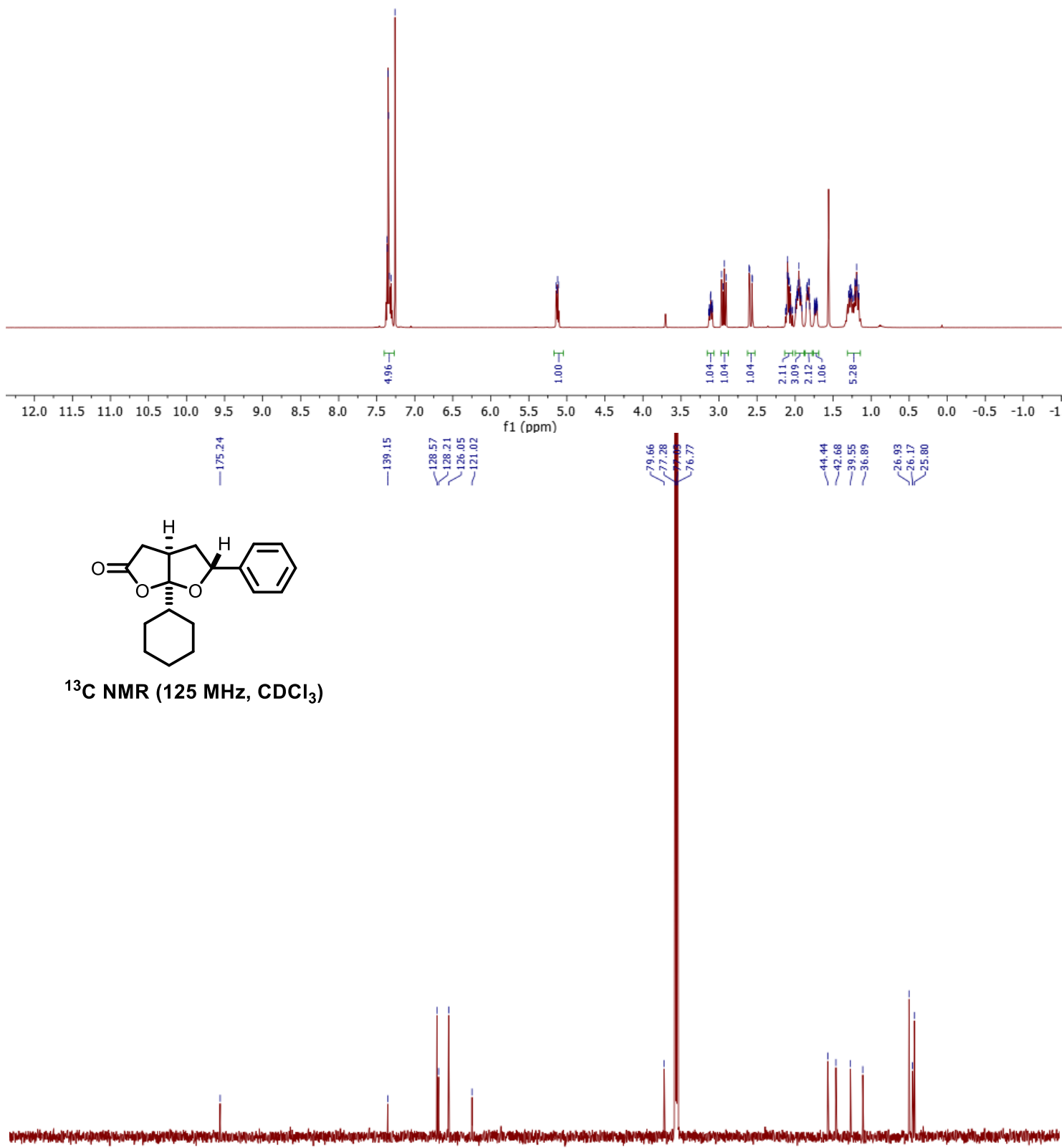

\begin{tabular}{lllllllllllllllllllllll}
\hline & 210 & 200 & 190 & 180 & 170 & 160 & 150 & 140 & 130 & 120 & 110 & 100 & 90 & 80 & 70 & 60 & 50 & 40 & 30 & 20 & 10 & 0
\end{tabular} 
<smiles>O=C1C[C@H]2CC(c3ccccc3)(O2)O[C@@]1(Cc1ccccc1)c1ccccc1</smiles>

${ }^{1} \mathrm{H}$ NMR $\left(500 \mathrm{MHz}, \mathrm{CDCl}_{3}\right)$

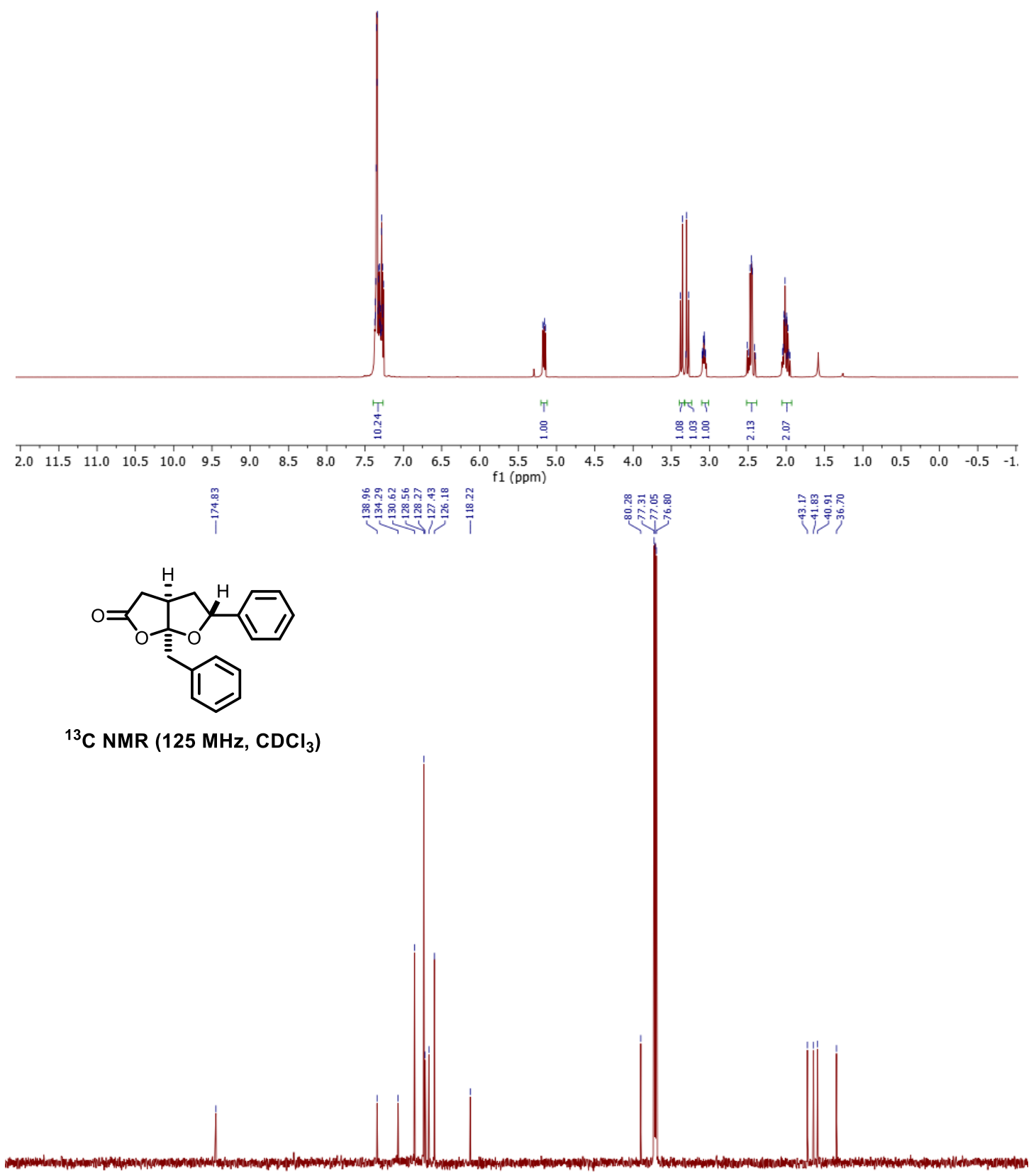

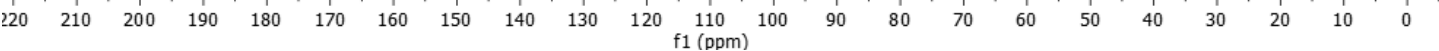




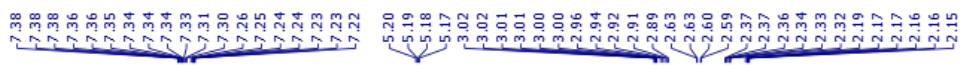
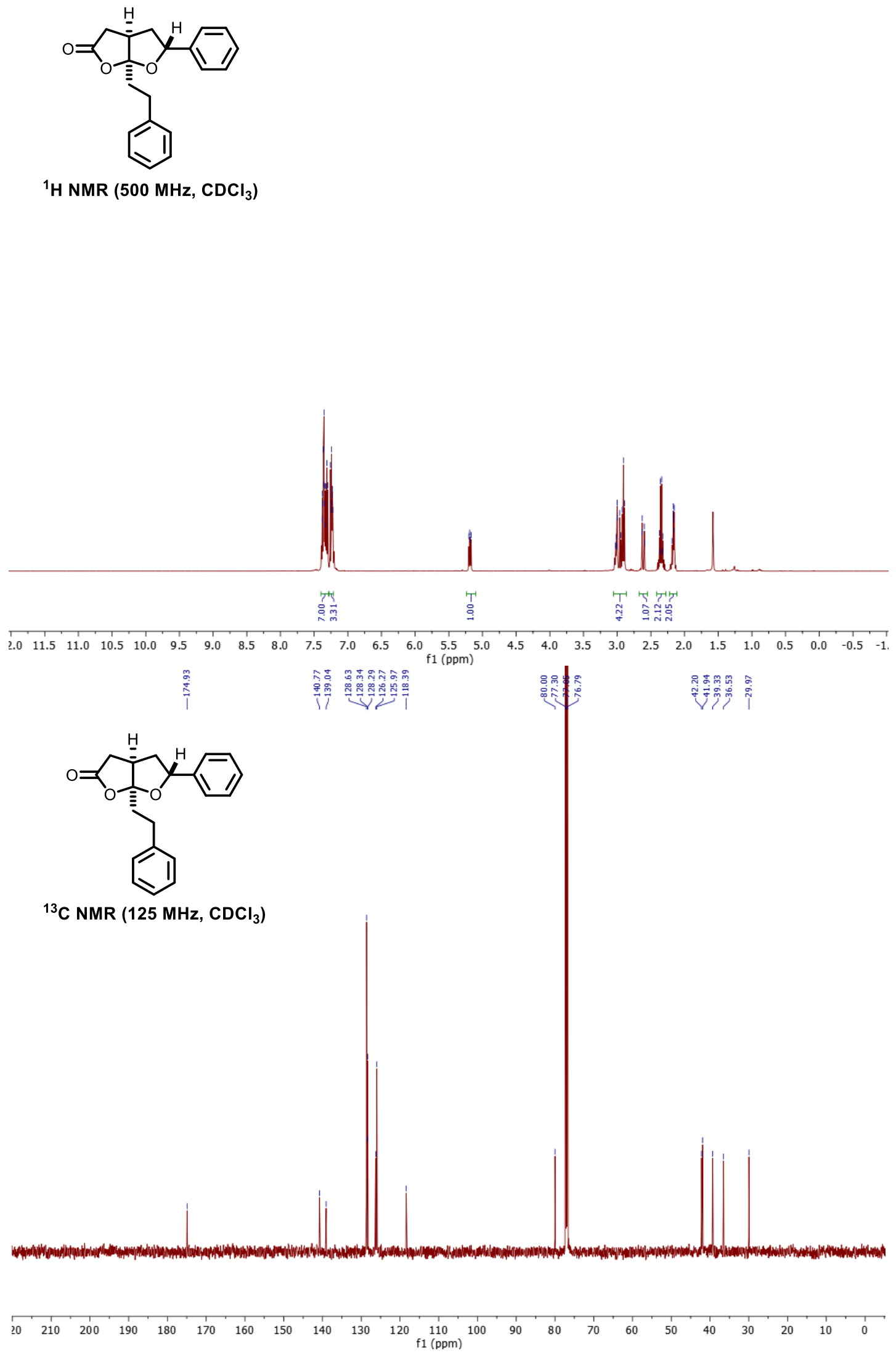

S90 


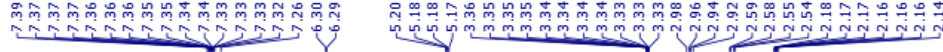<smiles>O=C1C[C@H]2C[C@@H](c3ccccc3)O[C@H]2O1</smiles>

${ }^{1} \mathrm{H}$ NMR $\left(500 \mathrm{MHz}, \mathrm{CDCl}_{3}\right)$
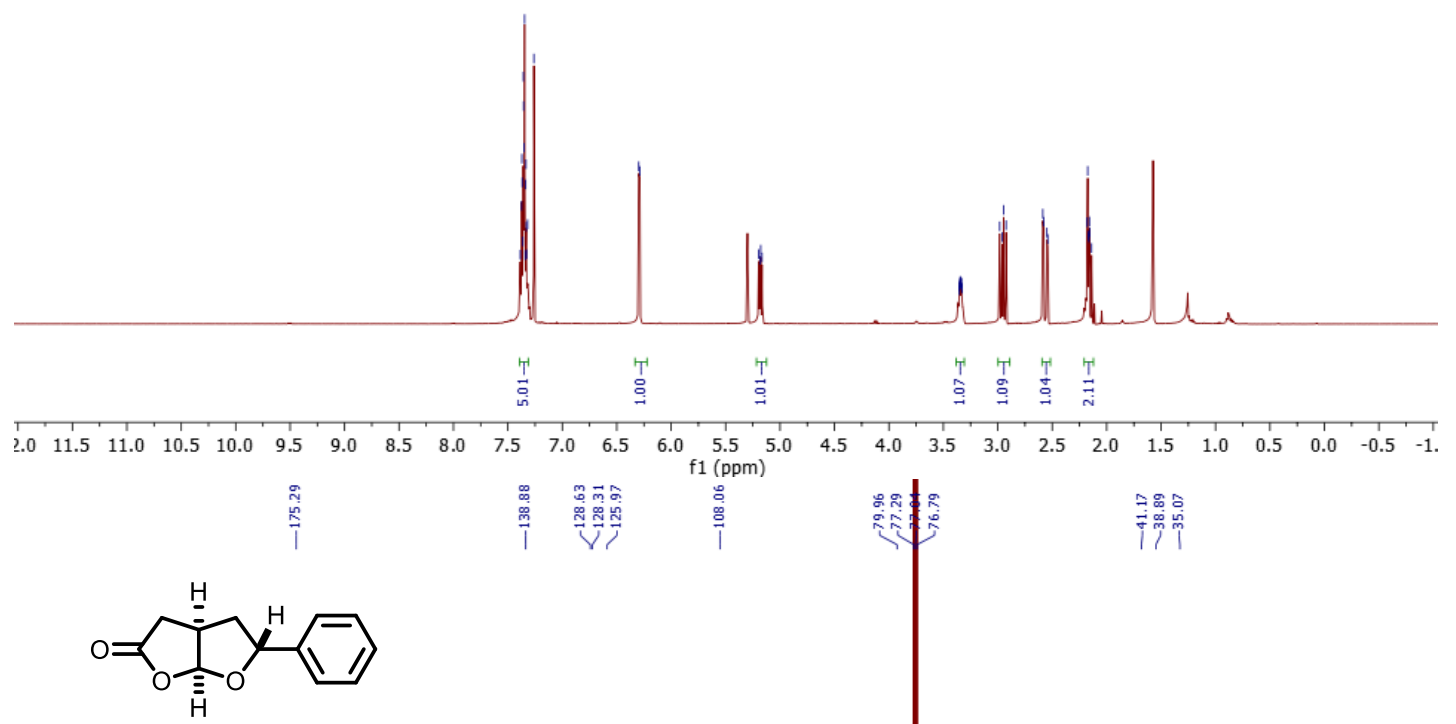

${ }^{13} \mathrm{C}$ NMR $\left(125 \mathrm{MHz}, \mathrm{CDCl}_{3}\right)$

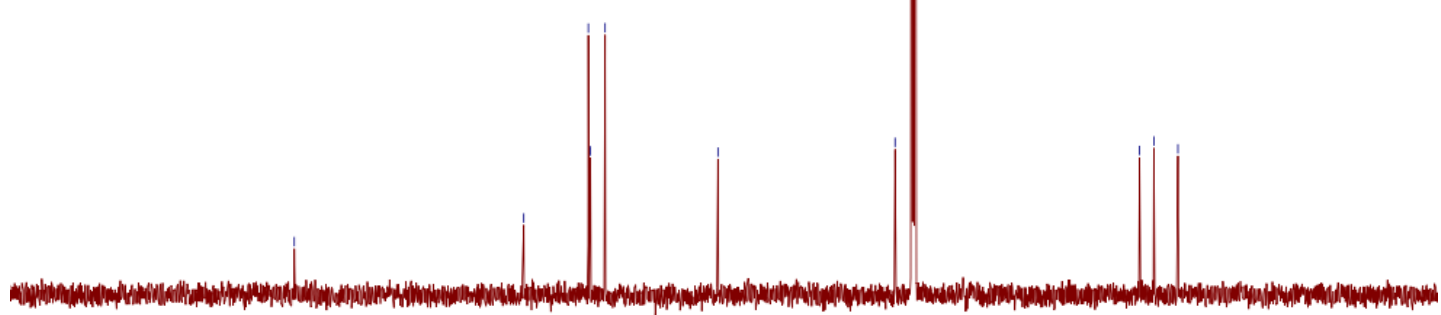

\begin{tabular}{lllllllllllllllllllllllll}
\hline 20 & 210 & 200 & 190 & 180 & 170 & 160 & 150 & 140 & 130 & 120 & 110 & 100 & 90 & 80 & 70 & 60 & 50 & 40 & 30 & 20 & 10 & 0
\end{tabular} 


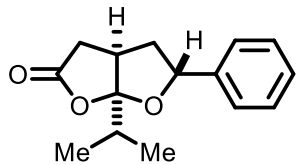

${ }^{1} \mathrm{H}$ NMR $\left(500 \mathrm{MHz}, \mathrm{CDCl}_{3}\right)$
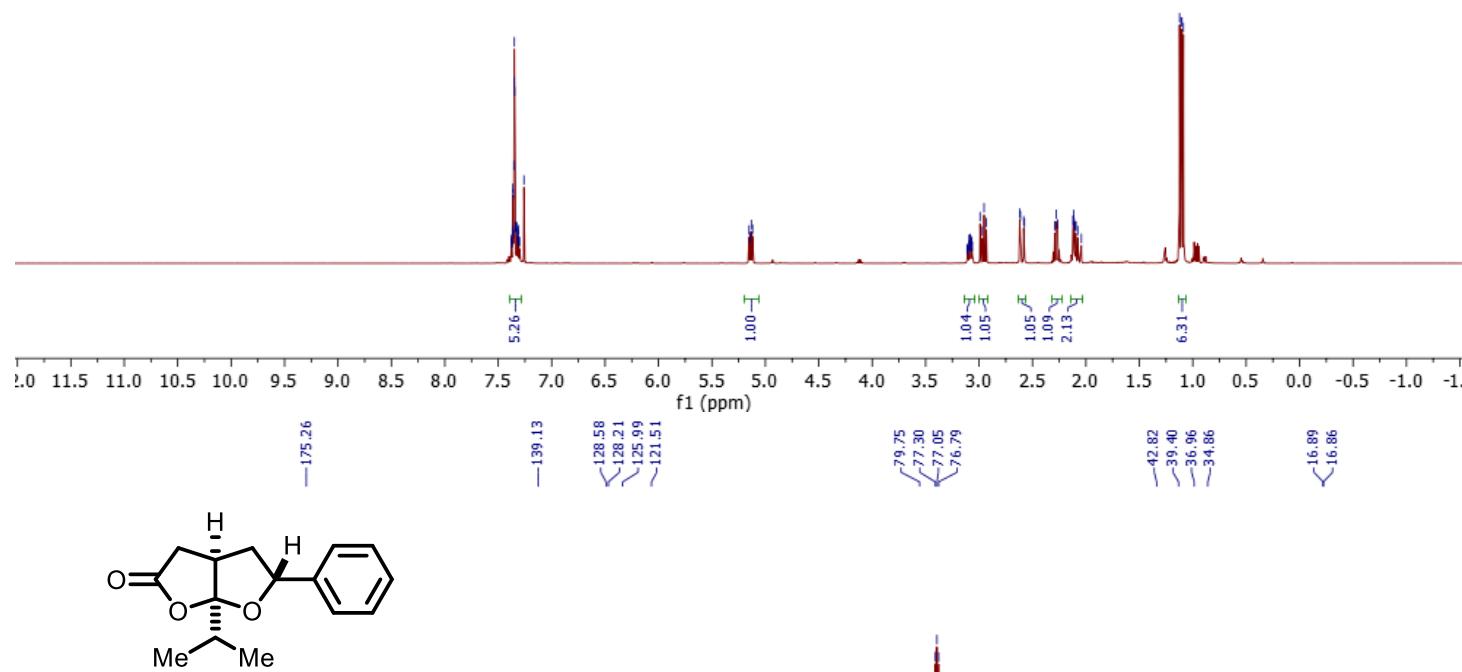

${ }^{13} \mathrm{C}$ NMR (125 MHz, $\left.\mathrm{CDCl}_{3}\right)$

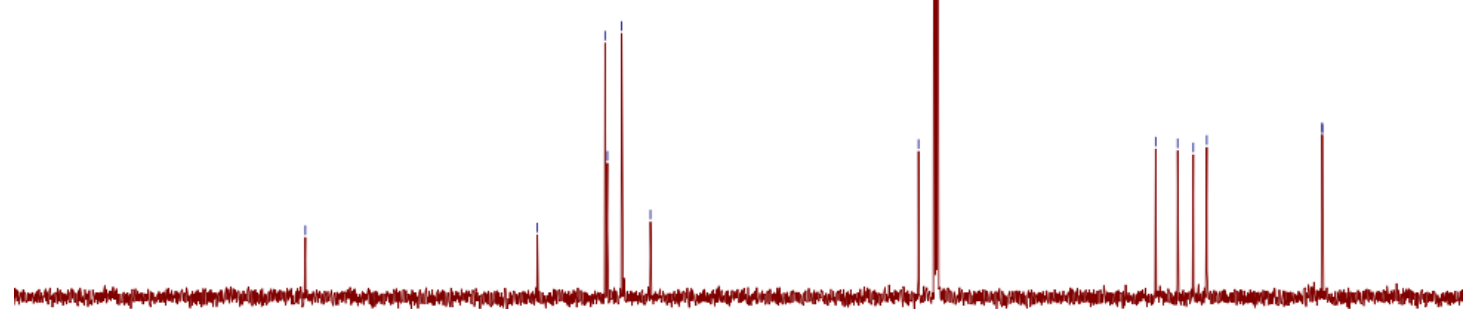

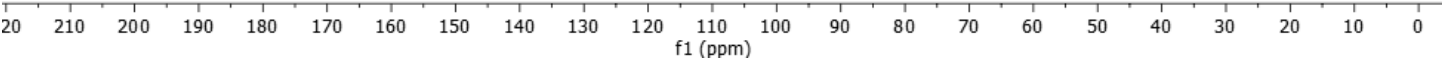



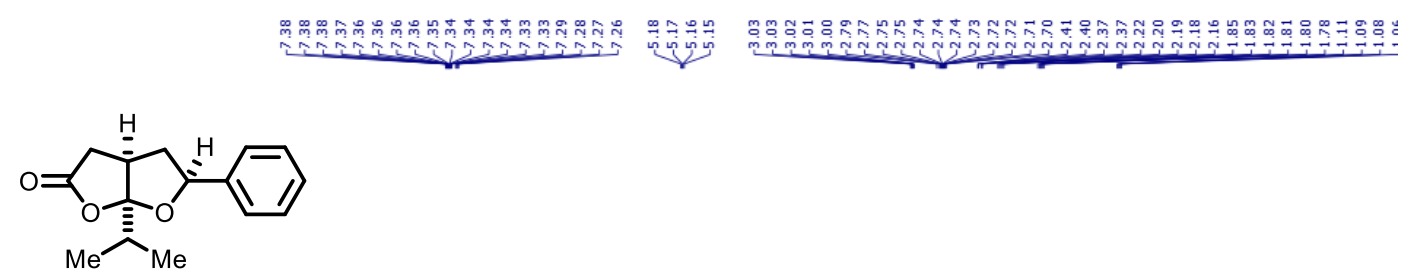

${ }^{1} \mathrm{H}$ NMR (500 MHz, $\left.\mathrm{CDCl}_{3}\right)$

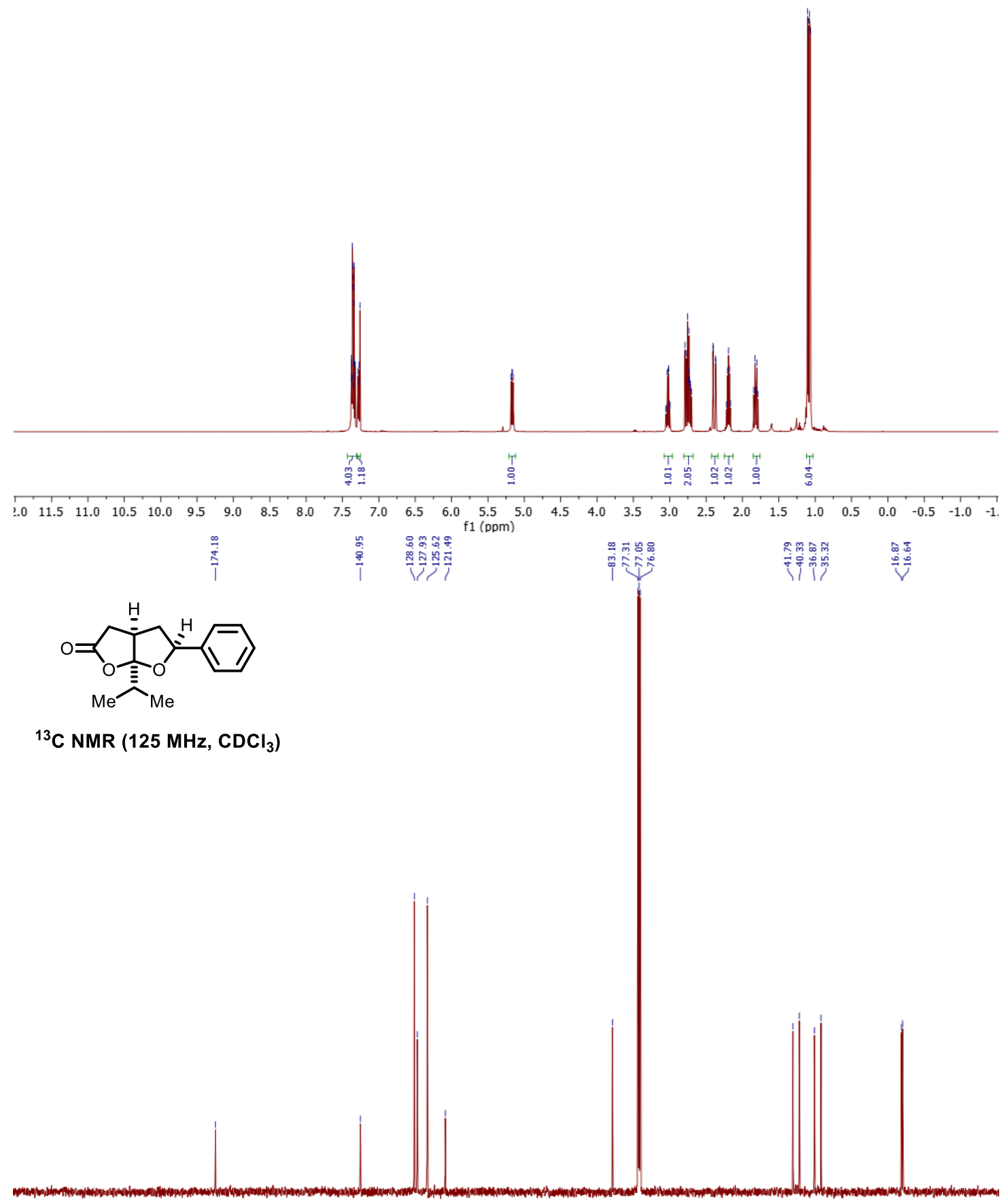

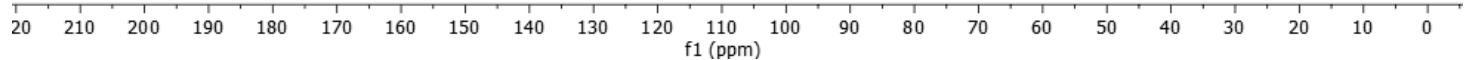




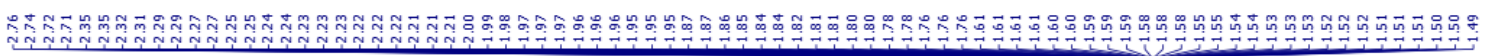

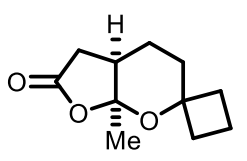

${ }^{1} \mathrm{H}$ NMR (500 MHz, $\left.\mathrm{CDCl}_{3}\right)$
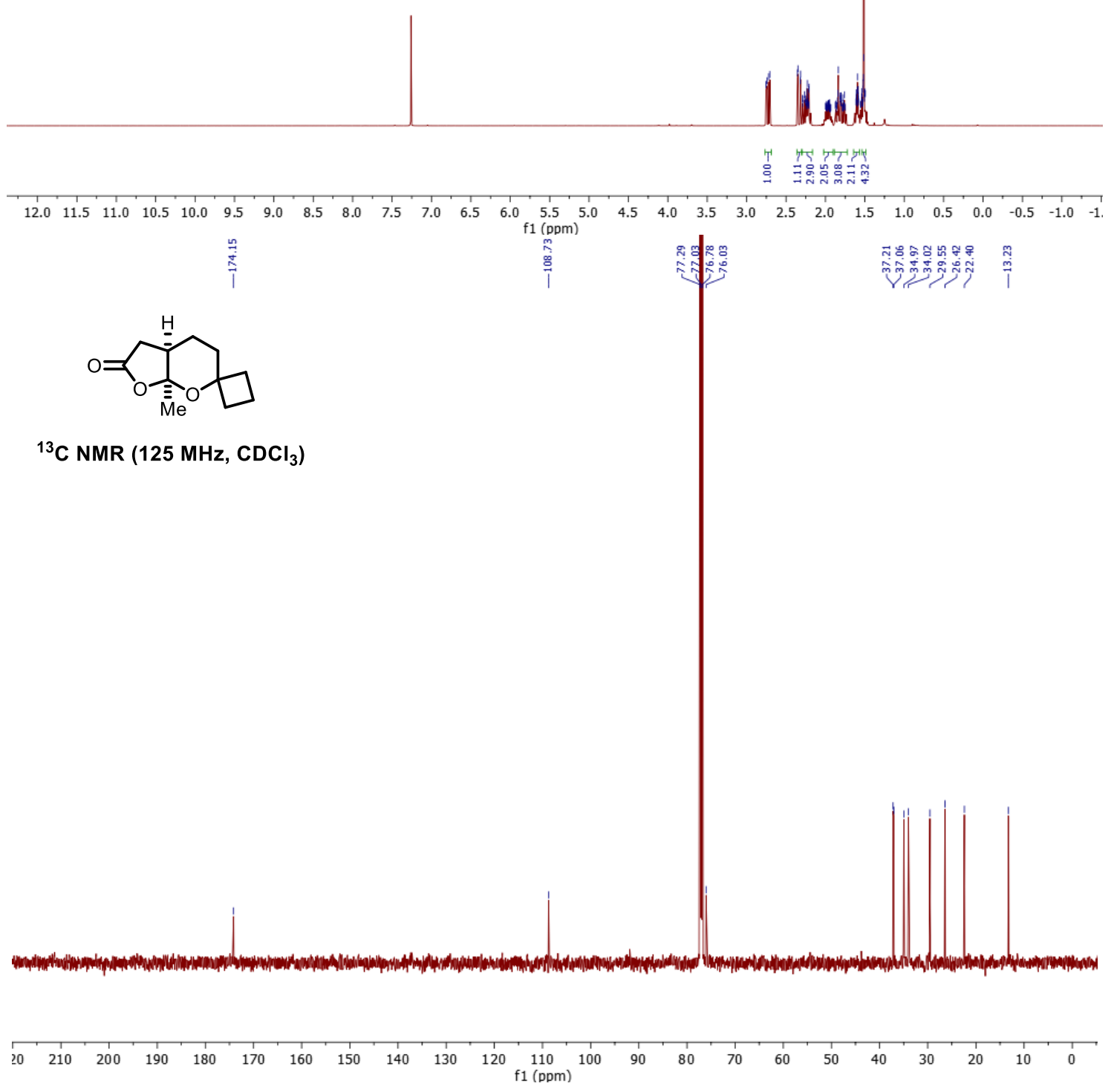

S94 
<smiles>C[C@]12OC(=O)C[C@H]1CCC1(CCCC1)O2</smiles>

${ }^{1} \mathrm{H}$ NMR (500 MHz, $\mathrm{CDCl}_{3}$ )
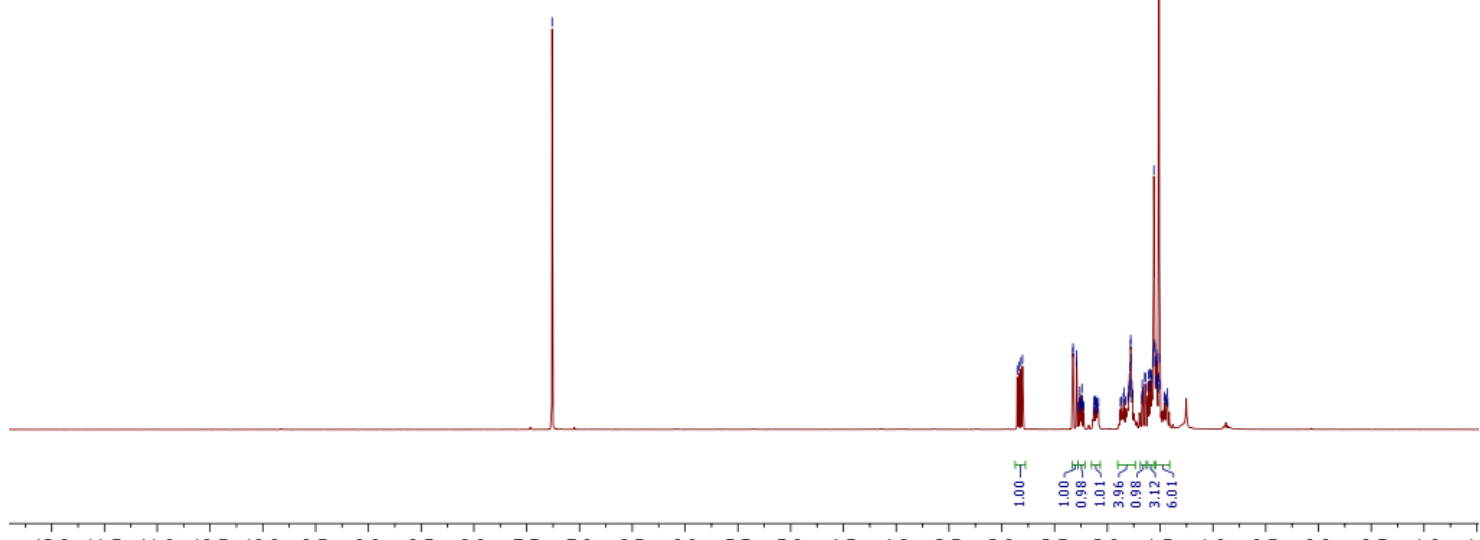
$\begin{array}{llllllllllllllllllllllllllllllll}12.0 & 11.5 & 11.0 & 10.5 & 10.0 & 9.5 & 9.0 & 8.5 & 8.0 & 7.5 & 7.0 & 6.5 & 6.0 & 5.5 & 5.0 & 4.5 & 4.0 & 3.5 & 3.0 & 2.5 & 2.0 & 1.5 & 1.0 & 0.5 & 0.0 & -0.5 & -1.0 & -1 .\end{array}$ I<smiles>C[C@]12OC(=O)C[C@H]1CCC1(CCCC1)O2</smiles>

${ }^{13} \mathrm{C}$ NMR $\left(125 \mathrm{MHz}, \mathrm{CDCl}_{3}\right)$

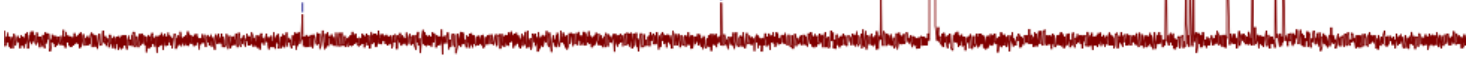

$\begin{array}{lllllllllllllllllllllllllll}20 & 210 & 200 & 190 & 180 & 170 & 160 & 150 & 140 & 130 & 120 & \begin{array}{c}110 \\ \mathrm{f} 1(\mathrm{ppm})\end{array} & 100 & 90 & 80 & 70 & 60 & 50 & 40 & 30 & 20 & 10 & 0\end{array}$ 
<smiles>C[C@]12OC(=O)C[C@H]1CCC1(CCCCC1)O2</smiles>

${ }^{1} \mathrm{H}$ NMR (500 MHz, $\left.\mathrm{CDCl}_{3}\right)$
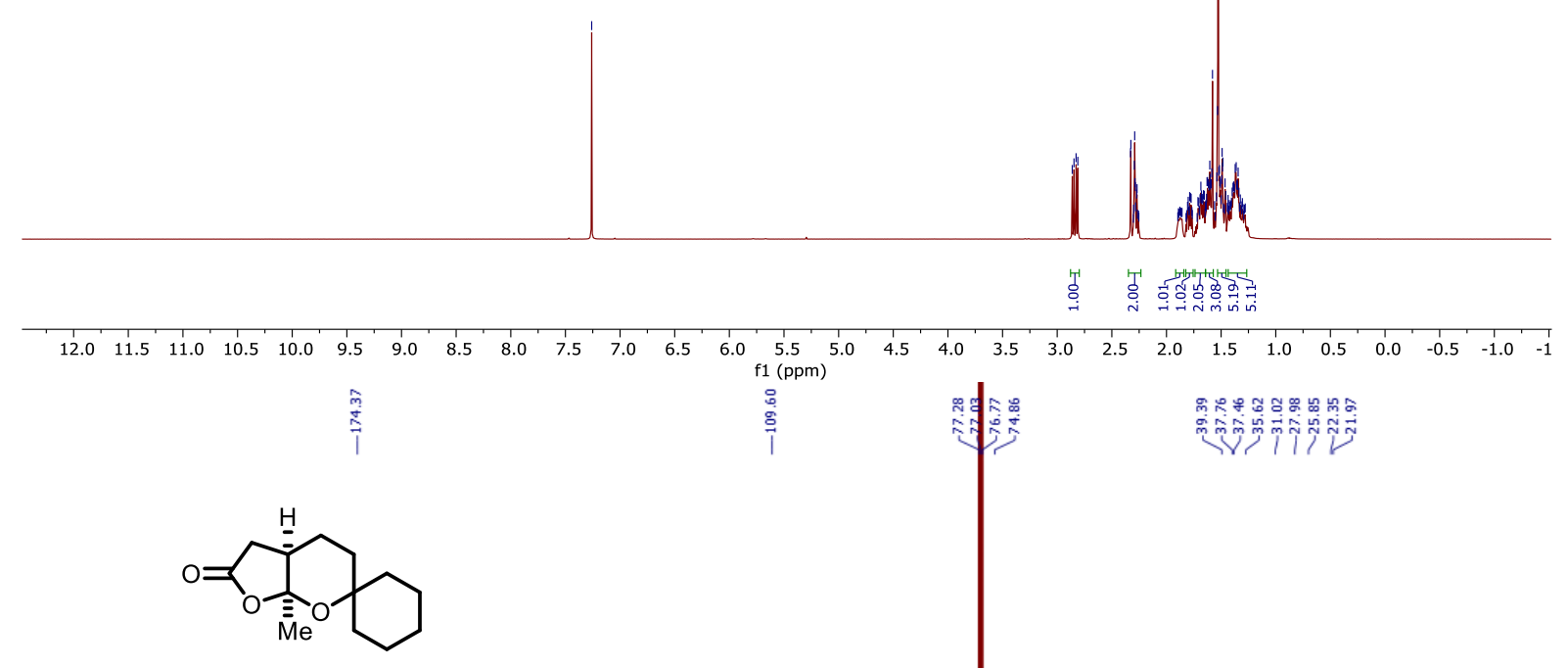

${ }^{13} \mathrm{C} \mathrm{NMR}\left(125 \mathrm{MHz}, \mathrm{CDCl}_{3}\right)$
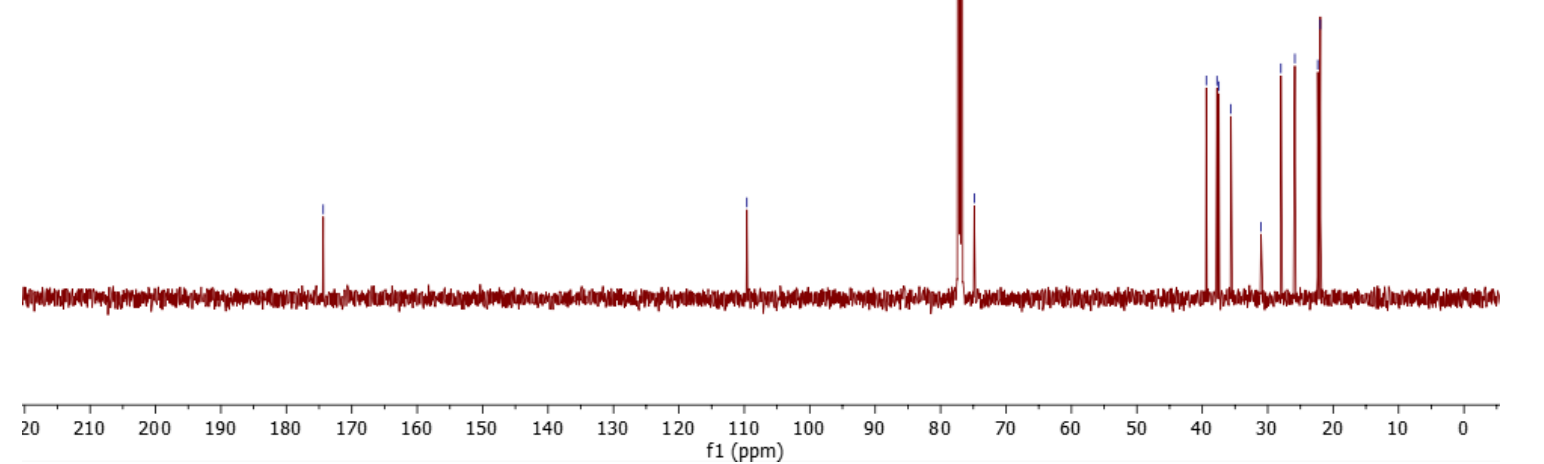
<smiles>C[C@]12OC(=O)C[C@H]1CC[C@H](c1ccccc1)O2</smiles>

${ }^{1} \mathrm{H}$ NMR (500 MHz, $\mathrm{CDCl}_{3}$ )

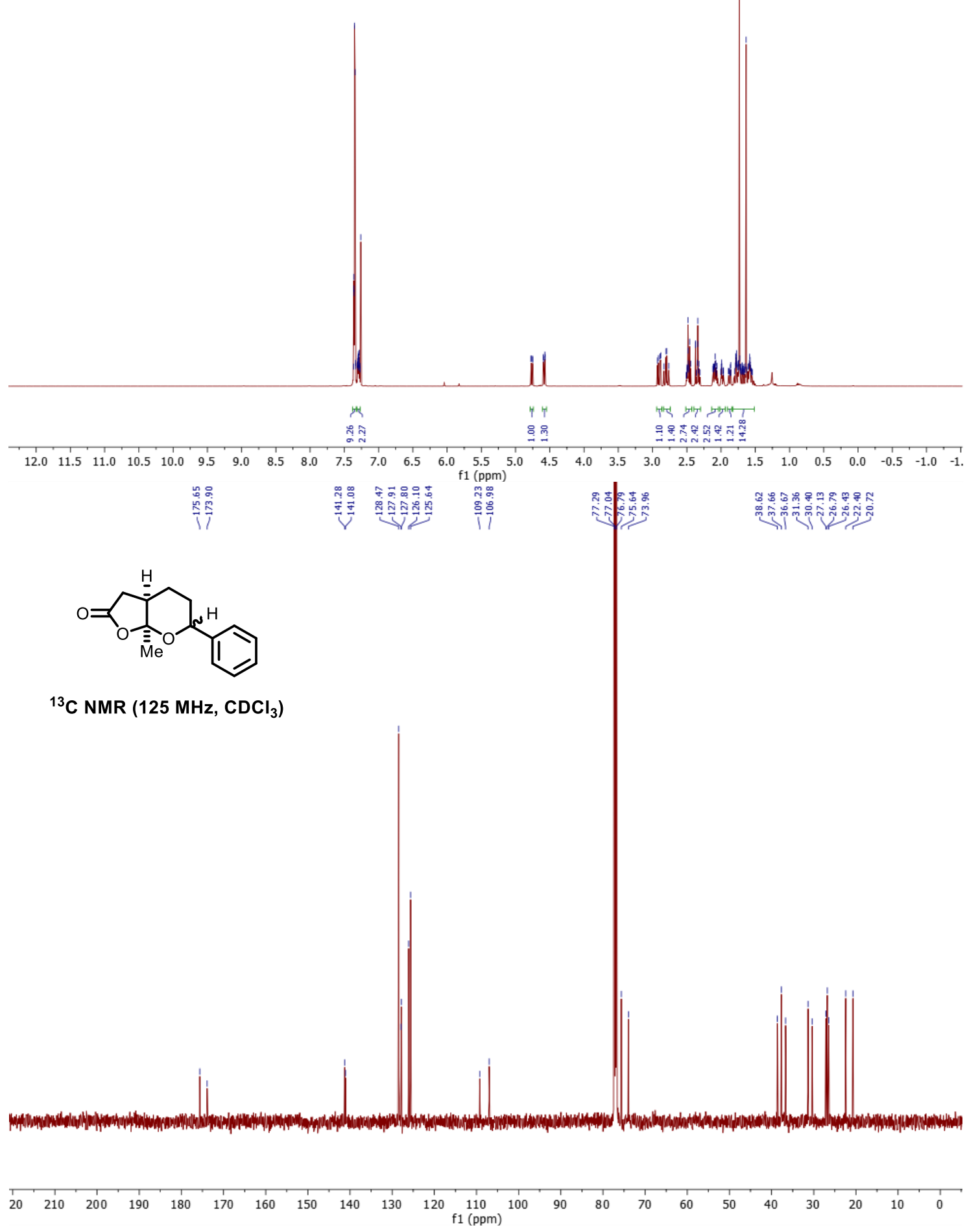




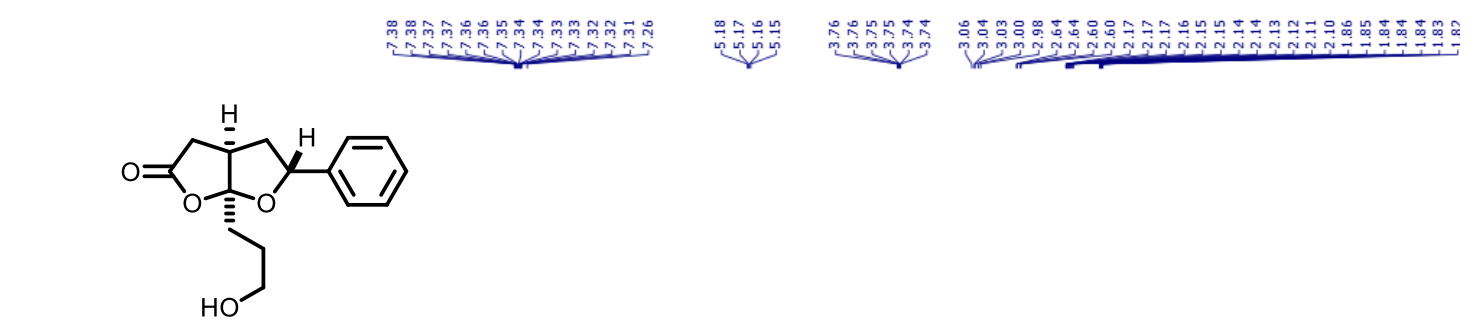

${ }^{1} \mathrm{H}$ NMR $\left(500 \mathrm{MHz}, \mathrm{CDCl}_{3}\right)$
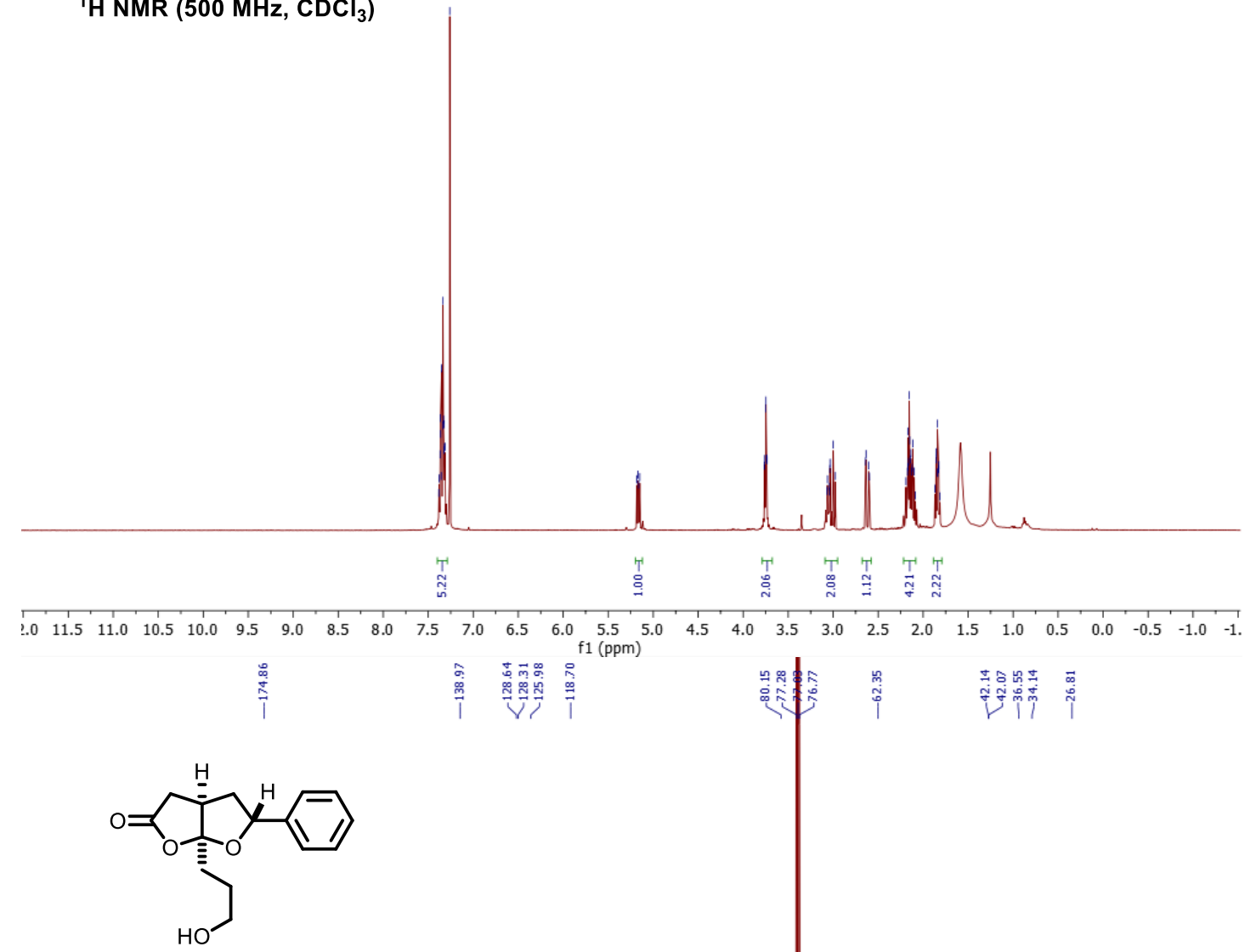

${ }^{13} \mathrm{C}$ NMR (125 MHz, $\left.\mathrm{CDCl}_{3}\right)$

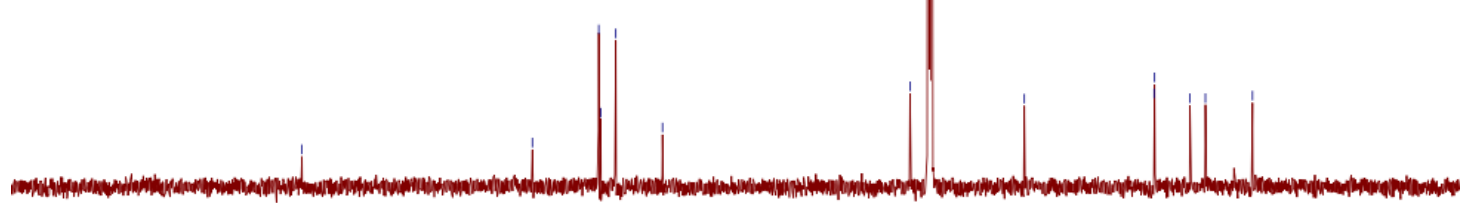

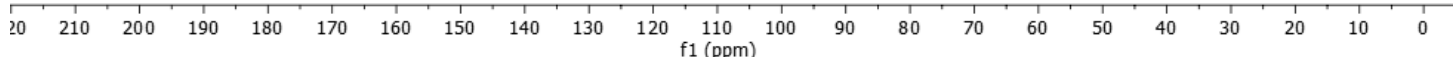




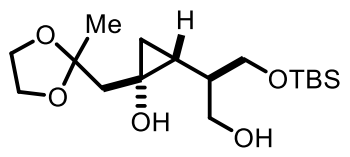

${ }^{1} \mathrm{H}$ NMR (500 MHz, $\left.\mathrm{CDCl}_{3}\right)$

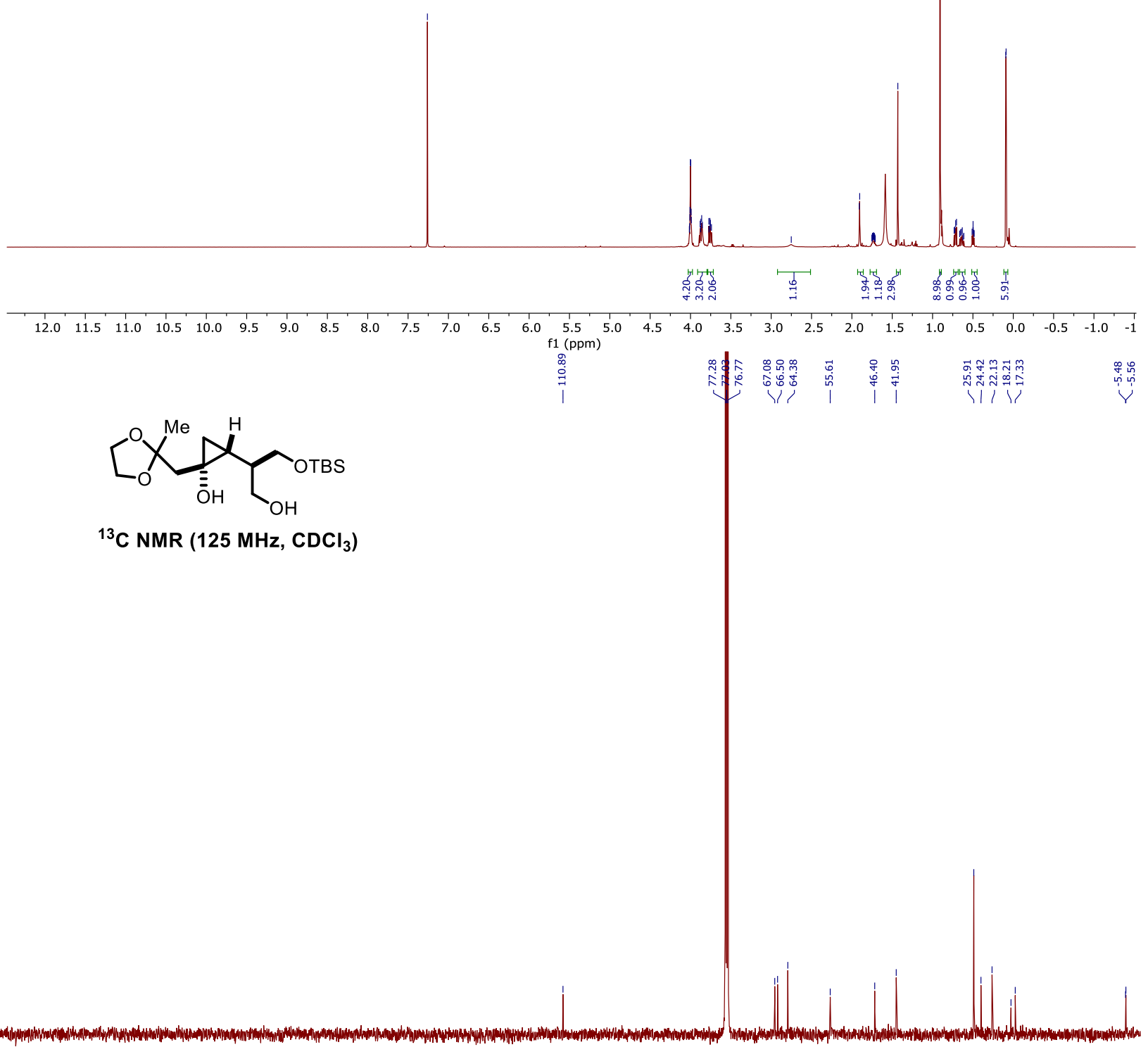

m.

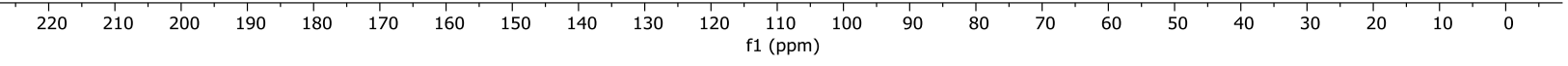




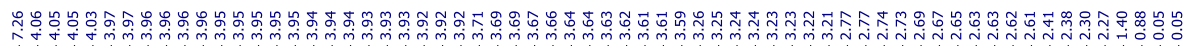

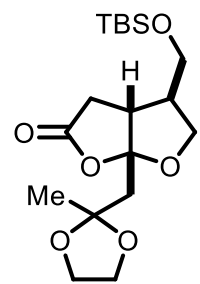

${ }^{1} \mathrm{H}$ NMR $\left(500 \mathrm{MHz}, \mathrm{CDCl}_{3}\right)$

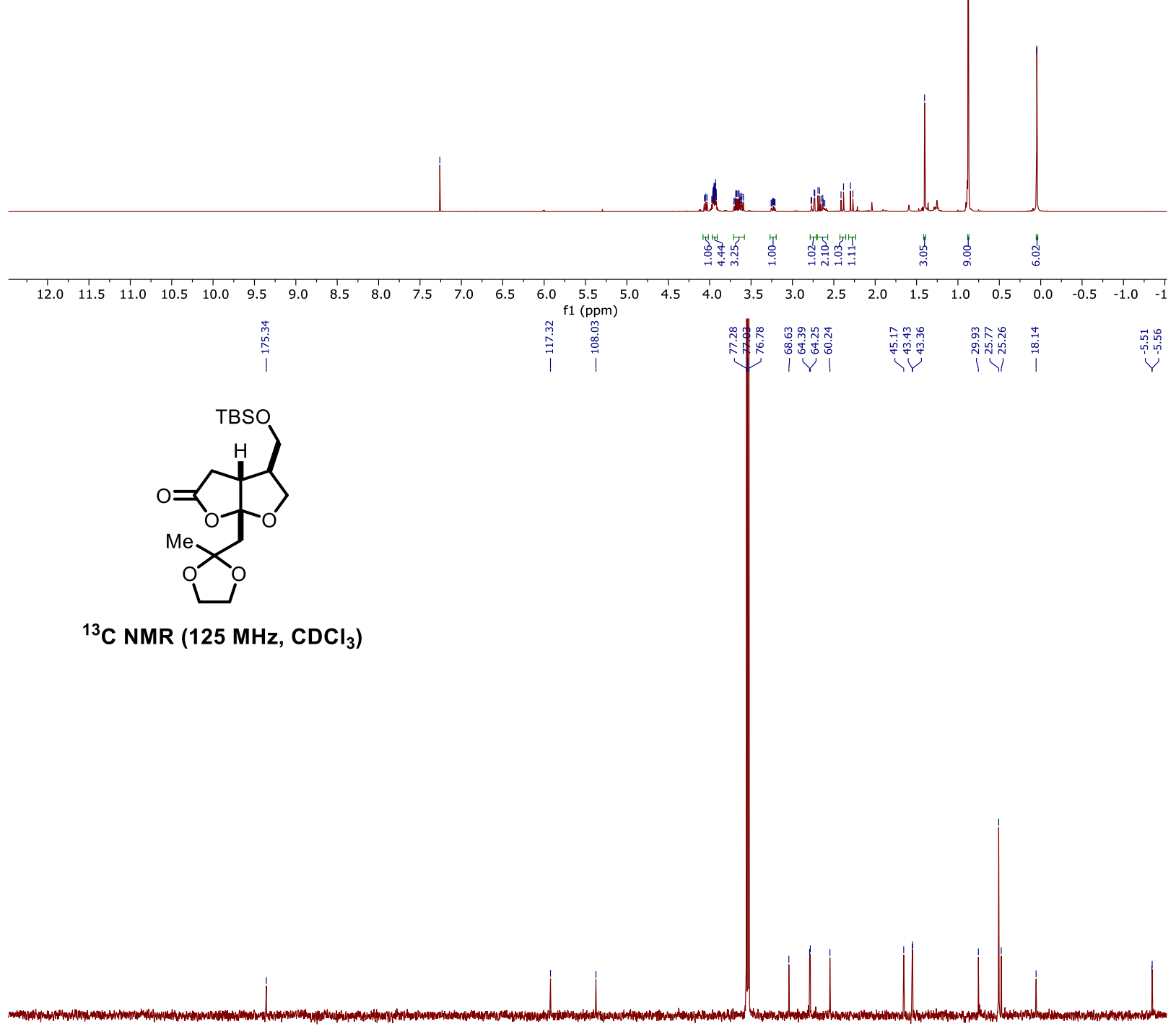

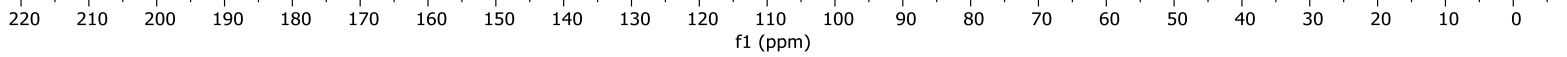



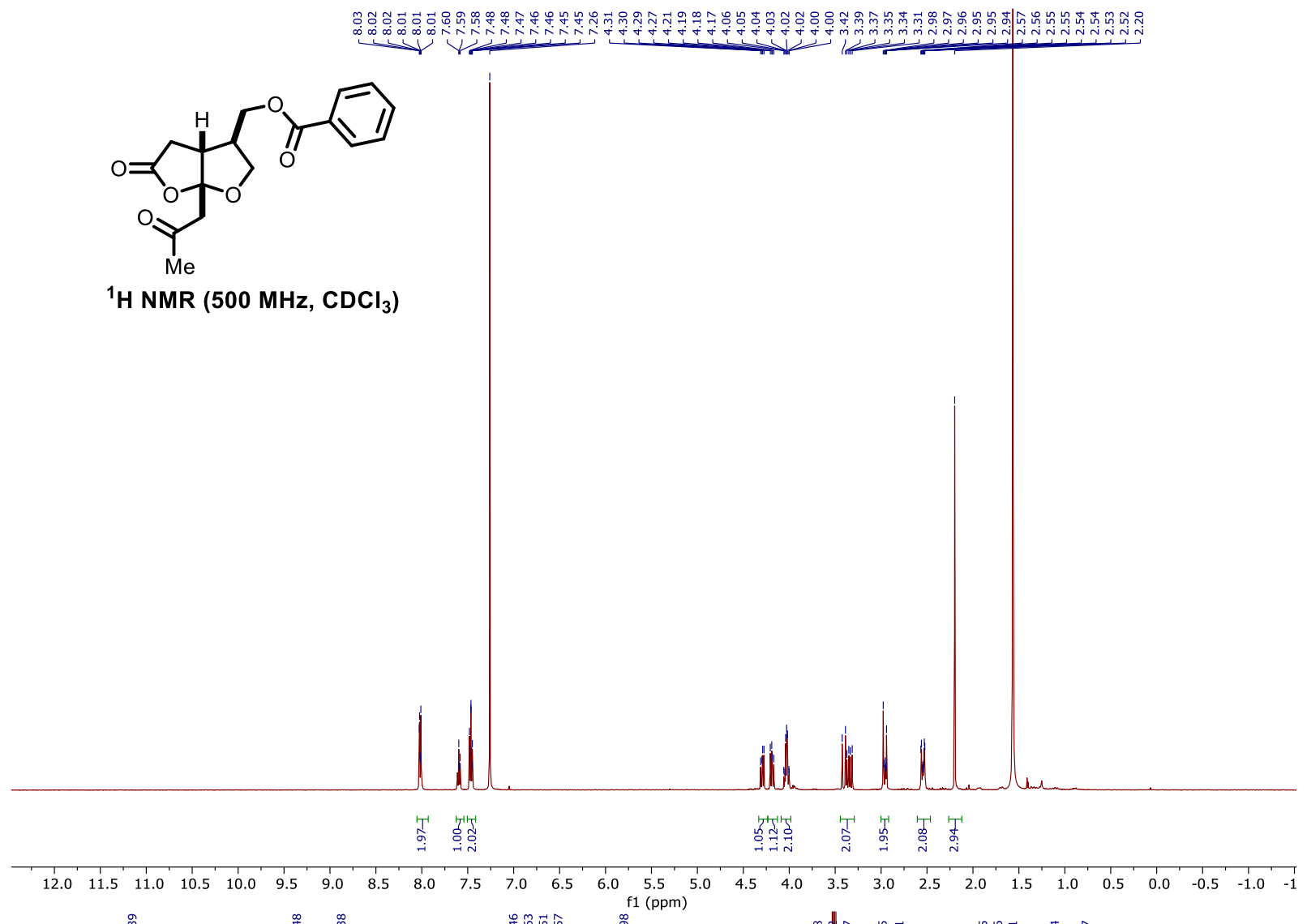

i

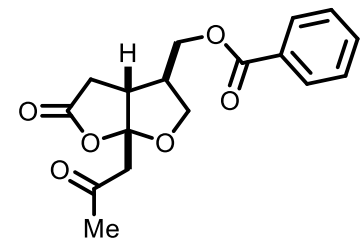

${ }^{13} \mathrm{C}$ NMR (125 MHz, $\left.\mathrm{CDCl}_{3}\right)$
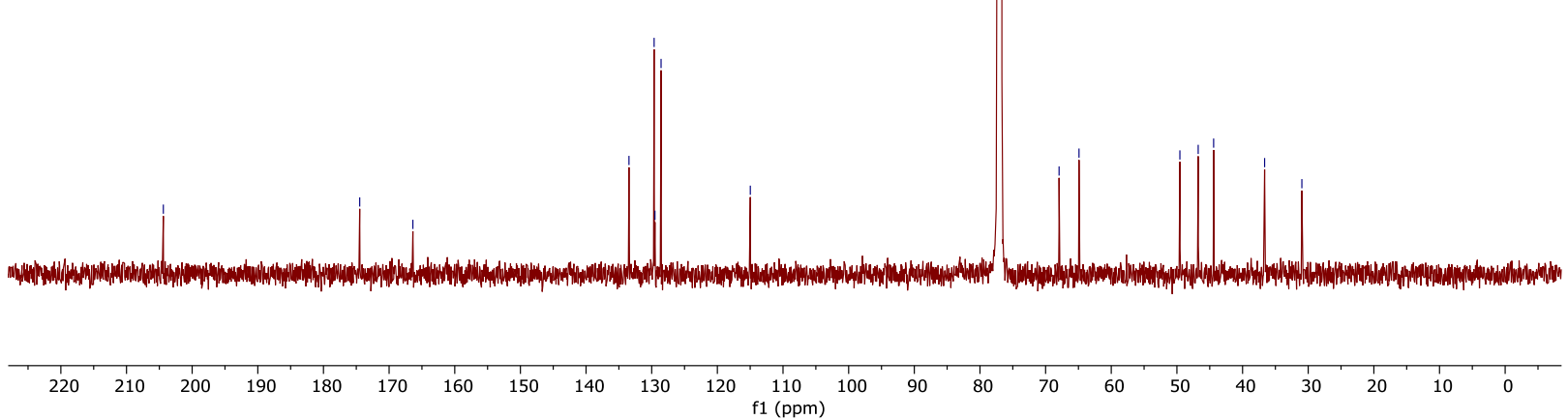


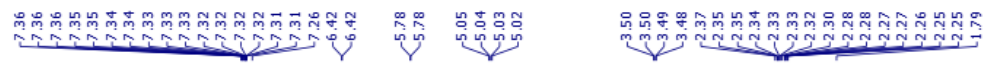

$O=\underbrace{\overline{\bar{M} e}}_{0} Y_{P h}^{H}$

${ }^{1} \mathrm{H}$ NMR $\left(500 \mathrm{MHz}, \mathrm{CDCl}_{3}\right)$
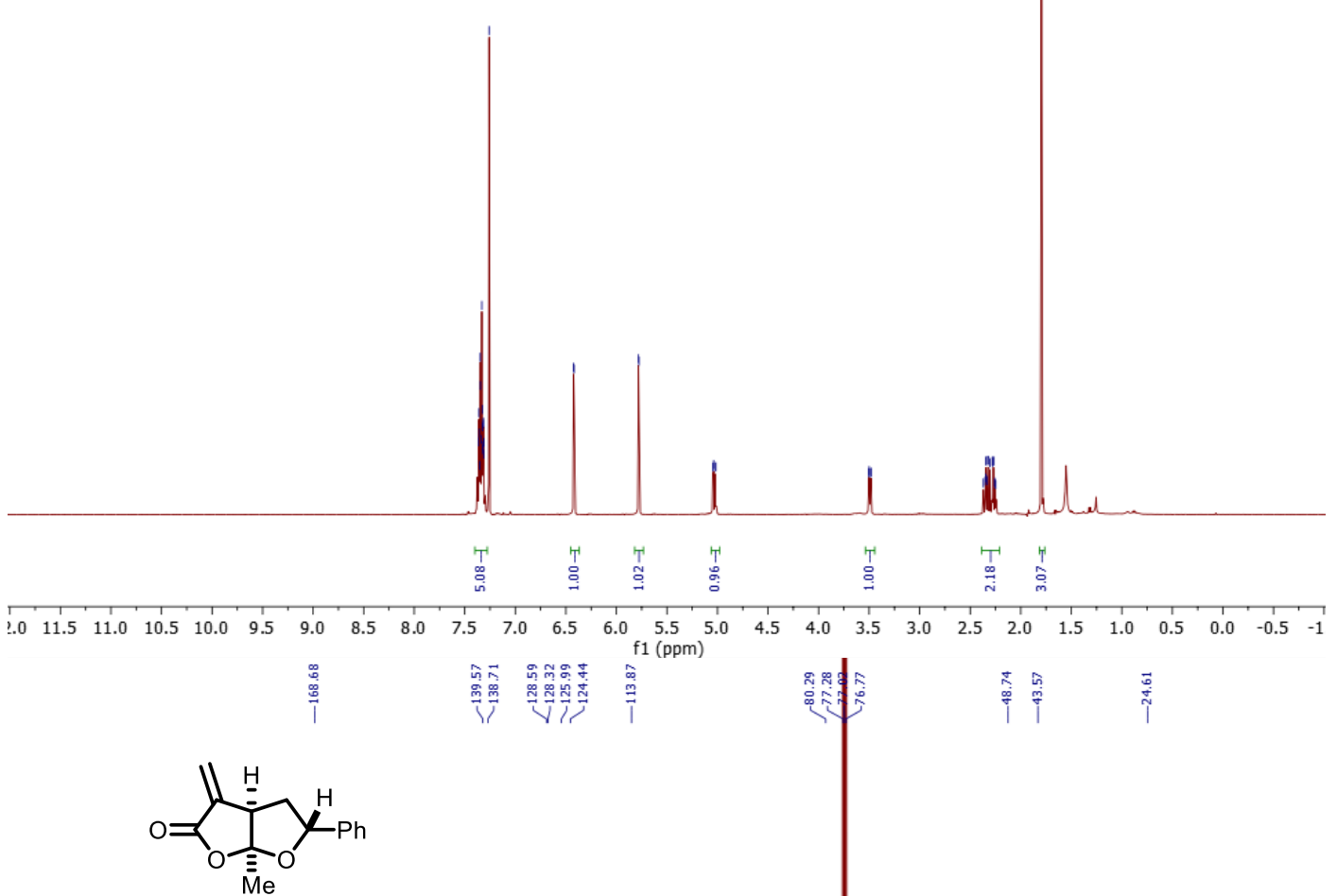

${ }^{13} \mathrm{C}$ NMR (125 MHz, $\mathrm{CDCl}_{3}$ )
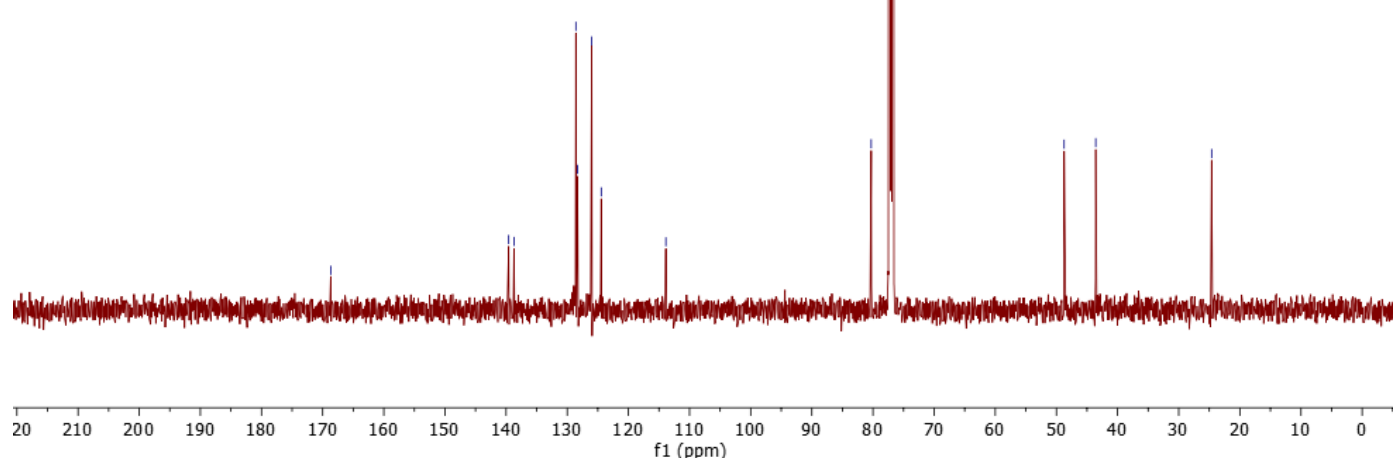

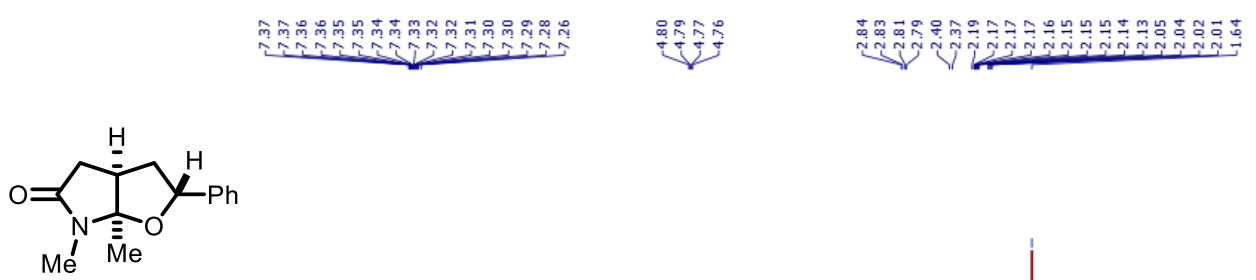

${ }^{1} \mathrm{H}$ NMR $\left(500 \mathrm{MHz}, \mathrm{CDCl}_{3}\right)$
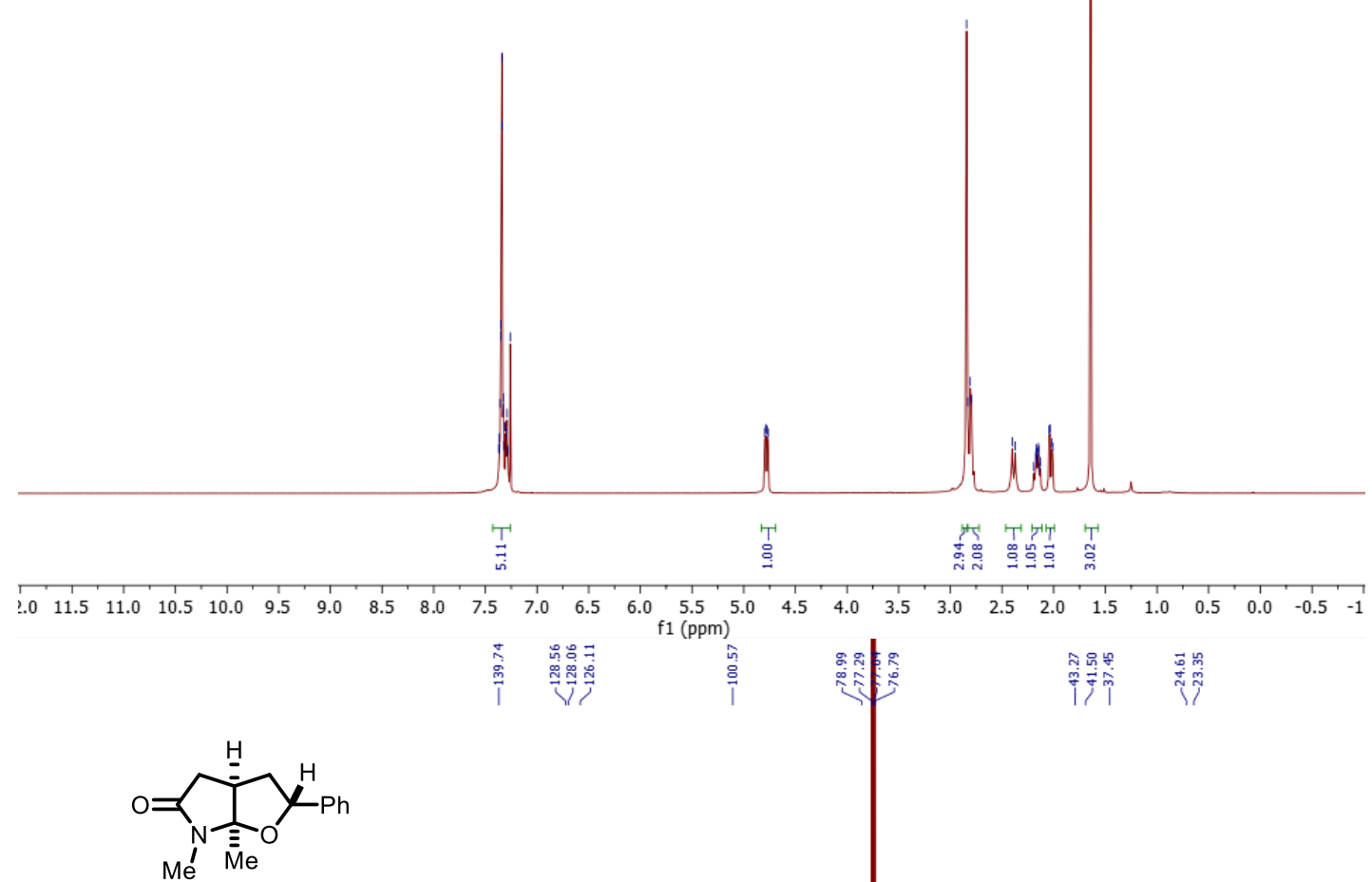

${ }^{13} \mathrm{C}$ NMR (125 MHz, $\left.\mathrm{CDCl}_{3}\right)$

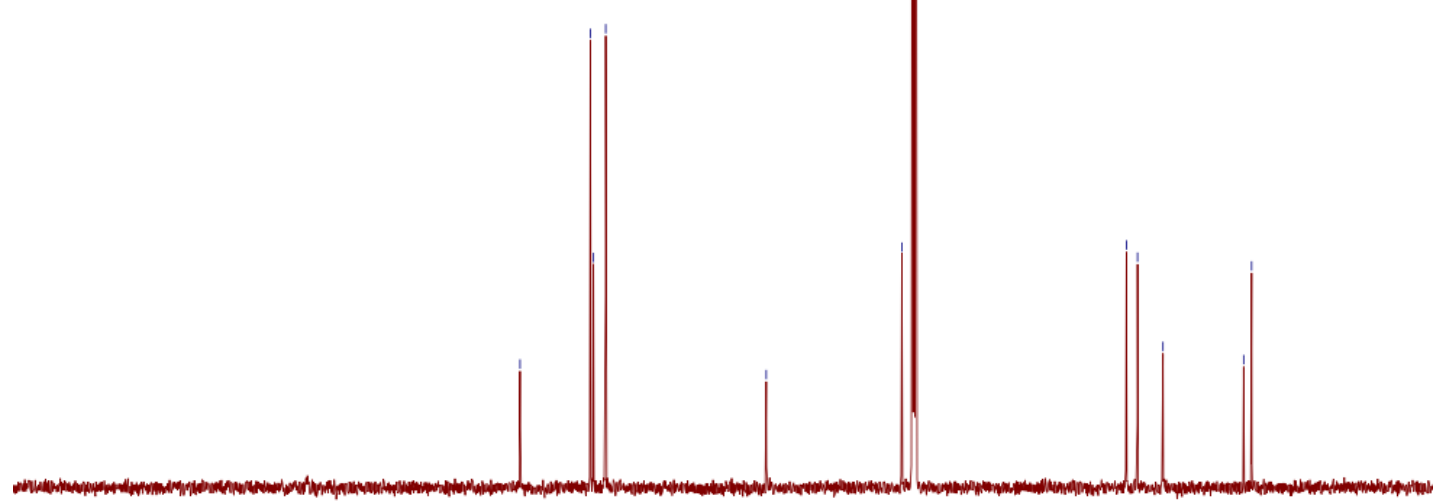

$\begin{array}{lllllllllllllllllllllll}20 & 210 & 200 & 190 & 180 & 170 & 160 & 150 & 140 & 130 & 120 & \begin{array}{c}110 \\ \mathrm{f} 1(\mathrm{ppm})\end{array} & 100 & 90 & 80 & 70 & 60 & 50 & 40 & 30 & 20 & 10 & 0\end{array}$ 


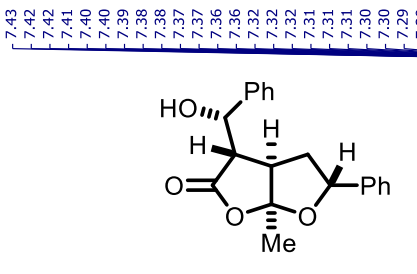

major diastereomer

${ }^{1} \mathrm{H}$ NMR (500 MHz, $\mathrm{CDCl}_{3}$ )

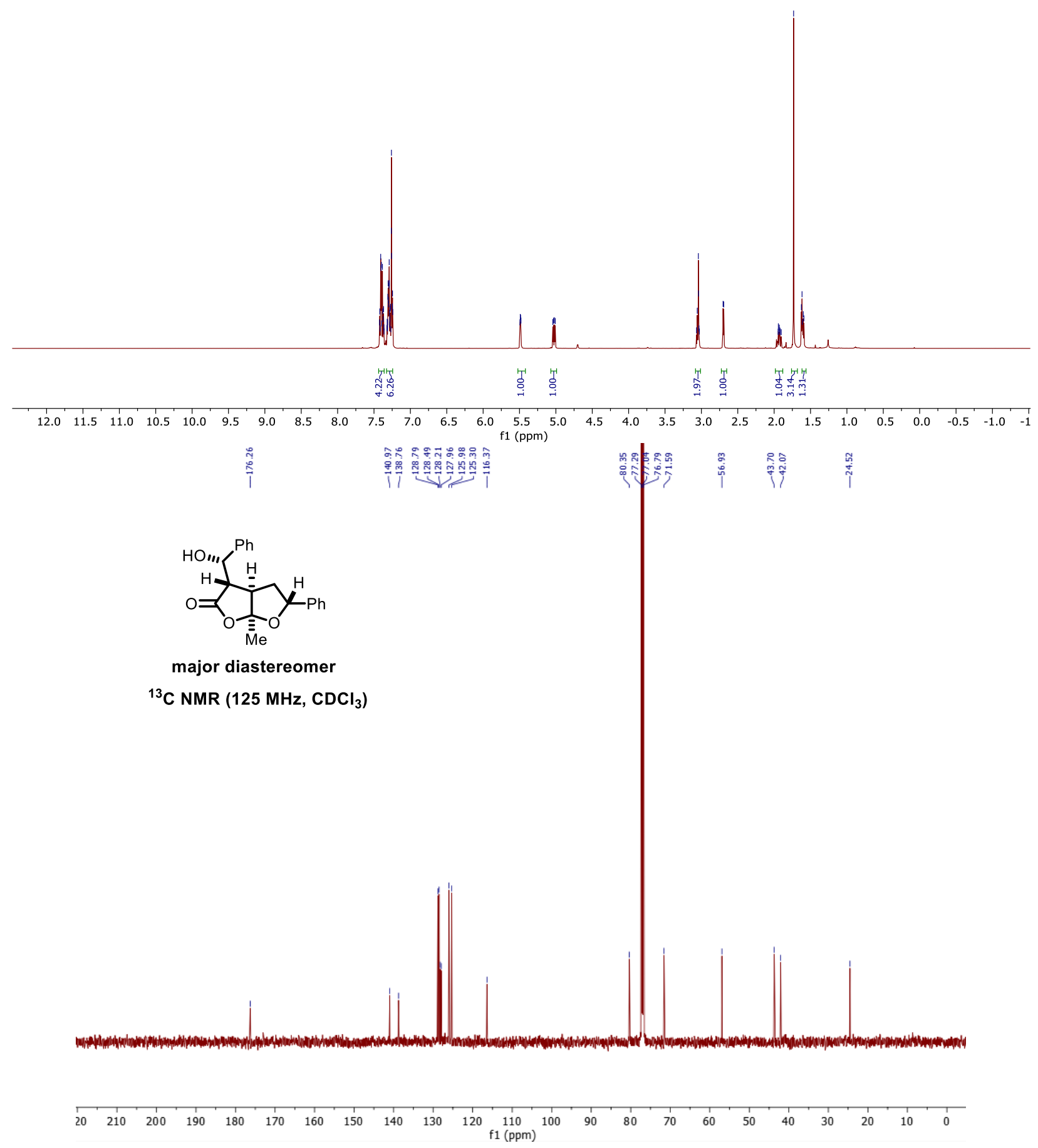




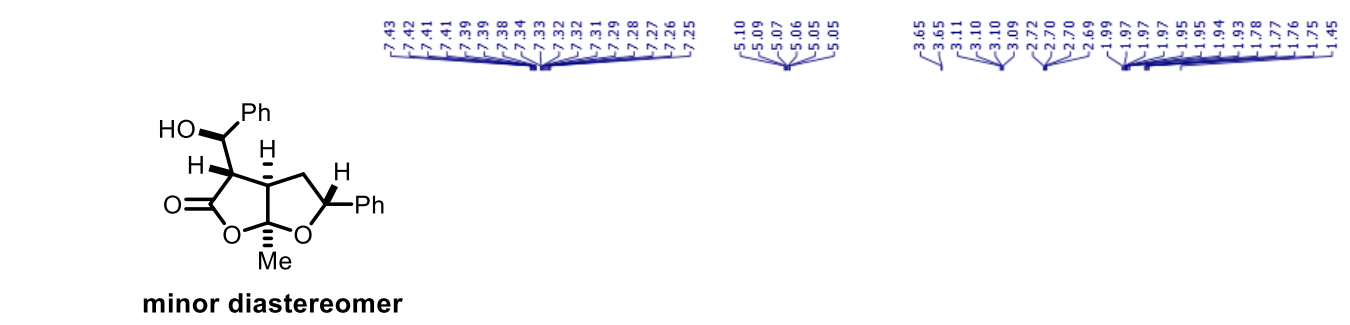

minor diastereomer
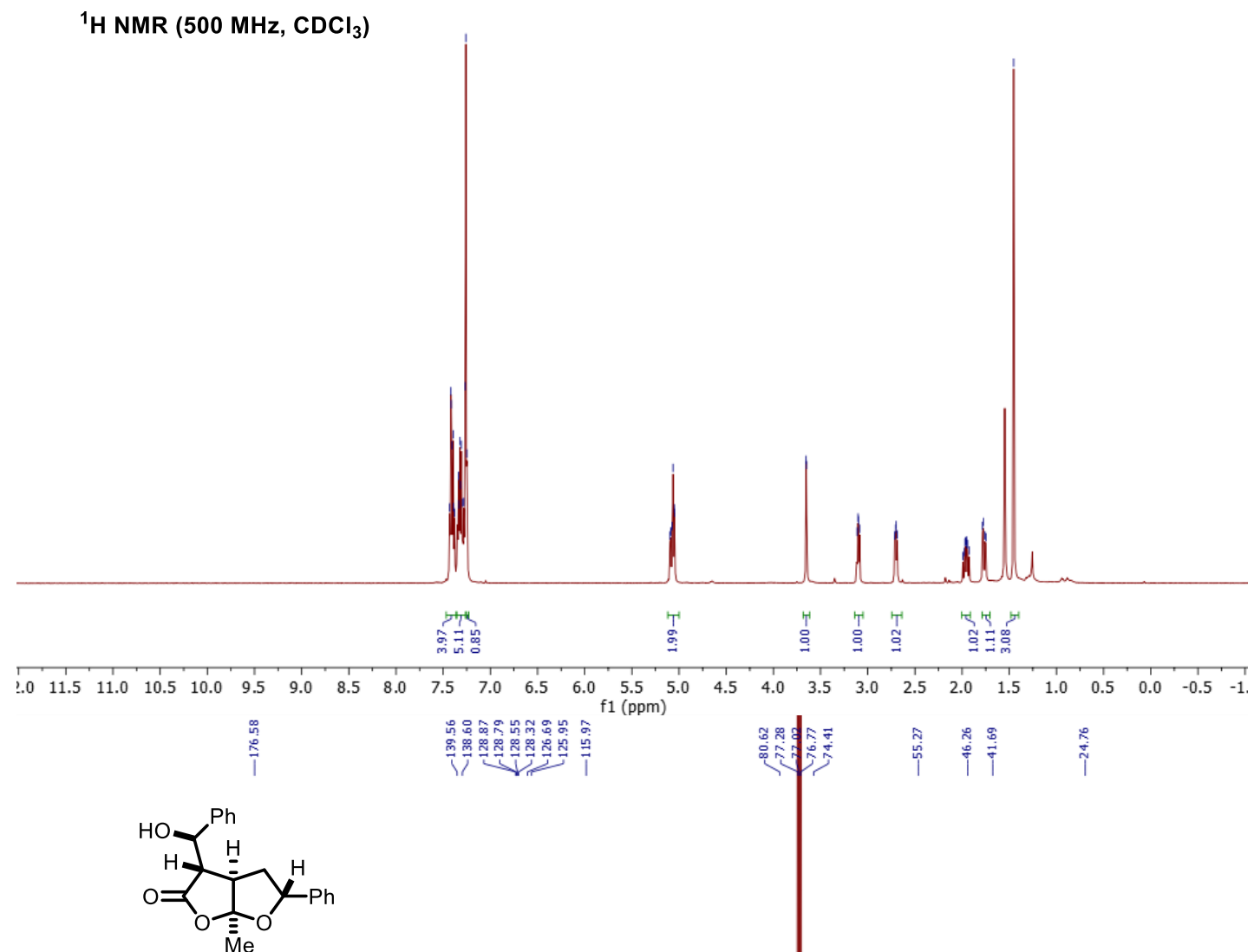

minor diastereomer

${ }^{13} \mathrm{C}$ NMR (125 MHz, $\left.\mathrm{CDCl}_{3}\right)$

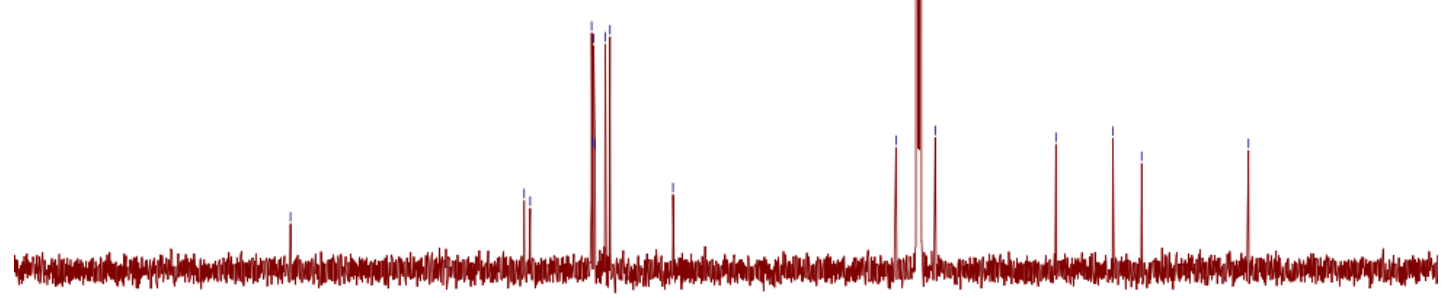

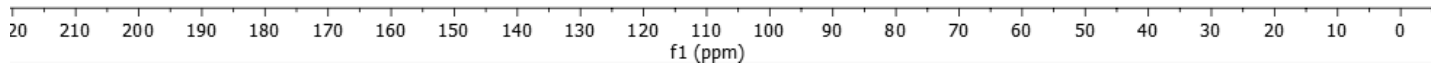




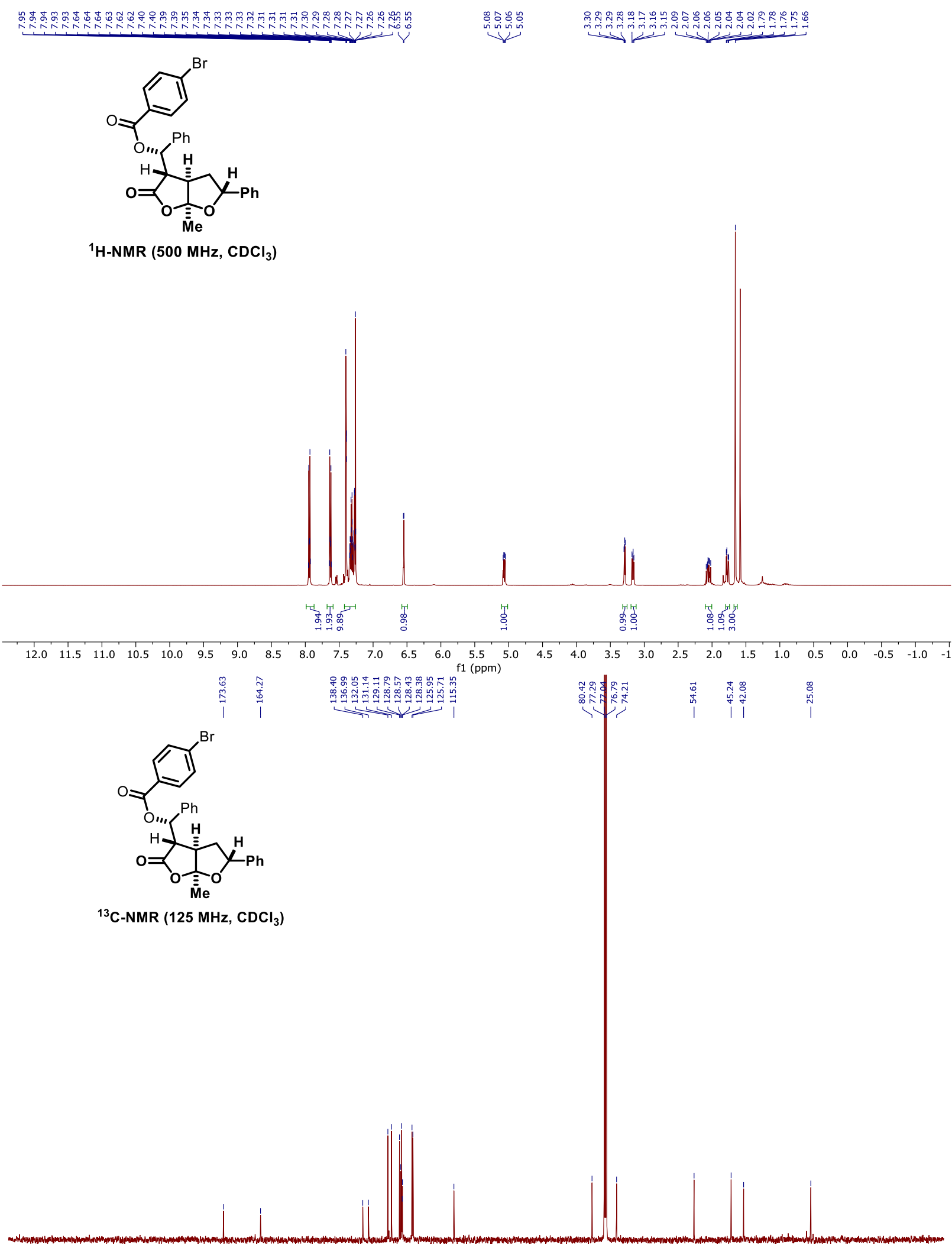

$\begin{array}{lllllllllllllllllllllllllll}220 & 210 & 200 & 190 & 180 & 170 & 160 & 150 & 140 & 130 & 120 & \begin{array}{c}110 \\ \mathrm{f} 1(\mathrm{ppm})\end{array} & 100 & 90 & 80 & 70 & 60 & 50 & 40 & 30 & 20 & 10 & 0\end{array}$ 


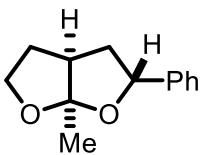

${ }^{1} \mathrm{H}$ NMR (500 MHz, $\mathrm{CDCl}_{3}$ )

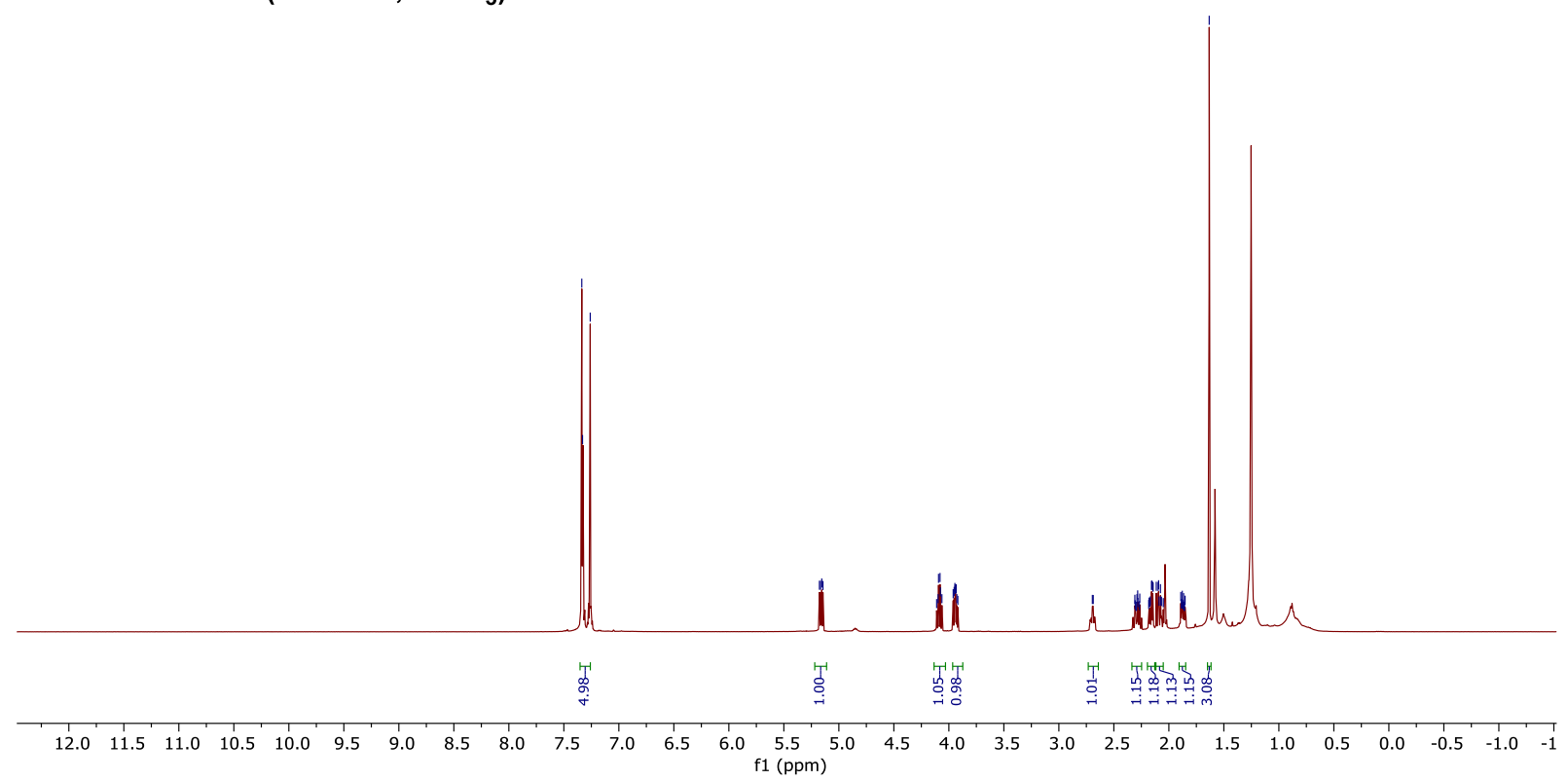




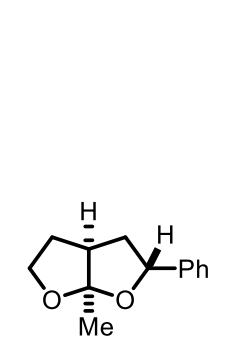

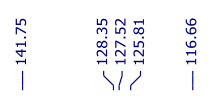

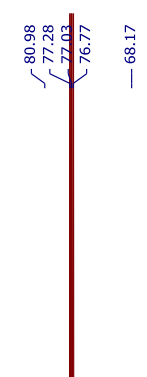

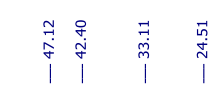

${ }^{13} \mathrm{C} \mathrm{NMR}\left(125 \mathrm{MHz}, \mathrm{CDCl}_{3}\right)$
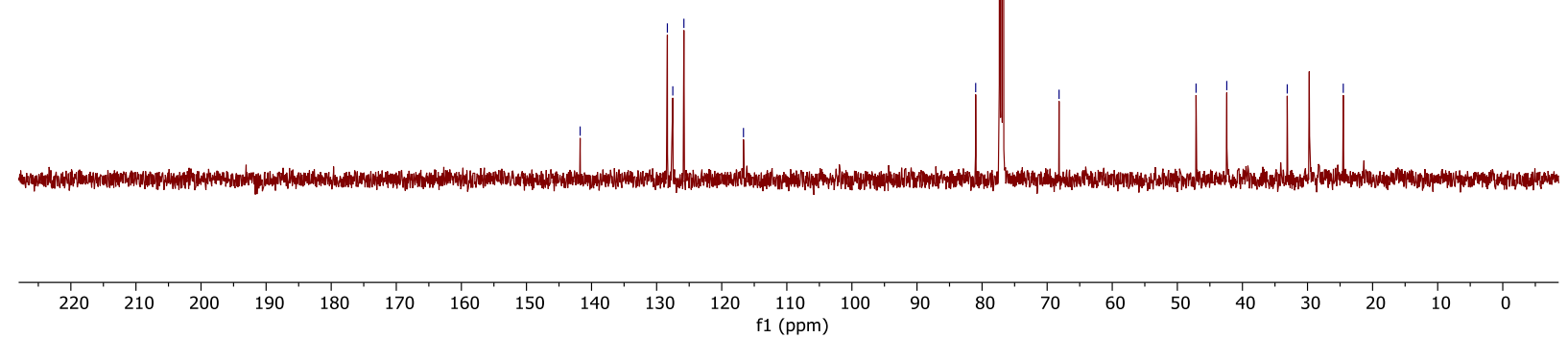


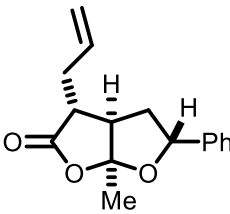

${ }^{1} \mathrm{H}$ NMR $\left(500 \mathrm{MHz}, \mathrm{CDCl}_{3}\right)$
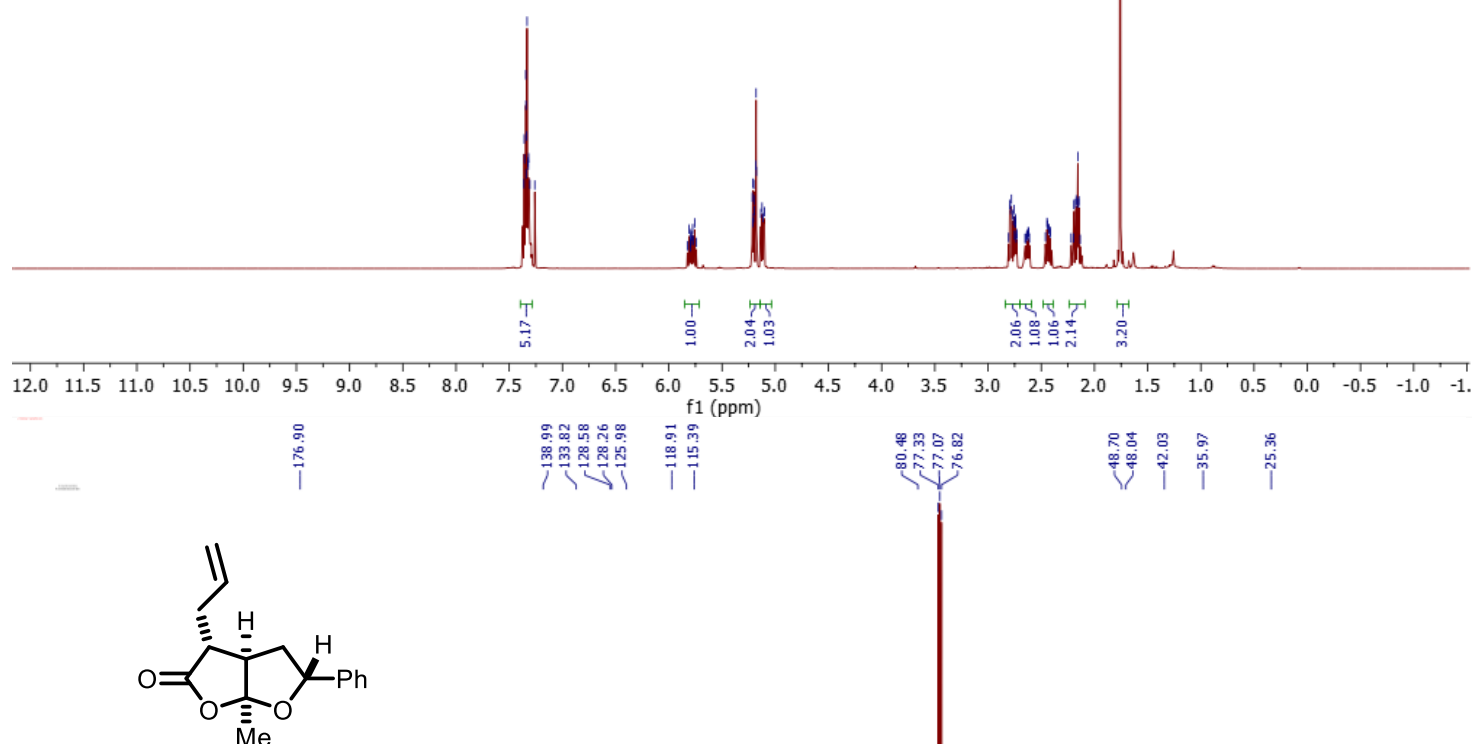

${ }^{13} \mathrm{C}$ NMR $\left(125 \mathrm{MHz}, \mathrm{CDCl}_{3}\right)$

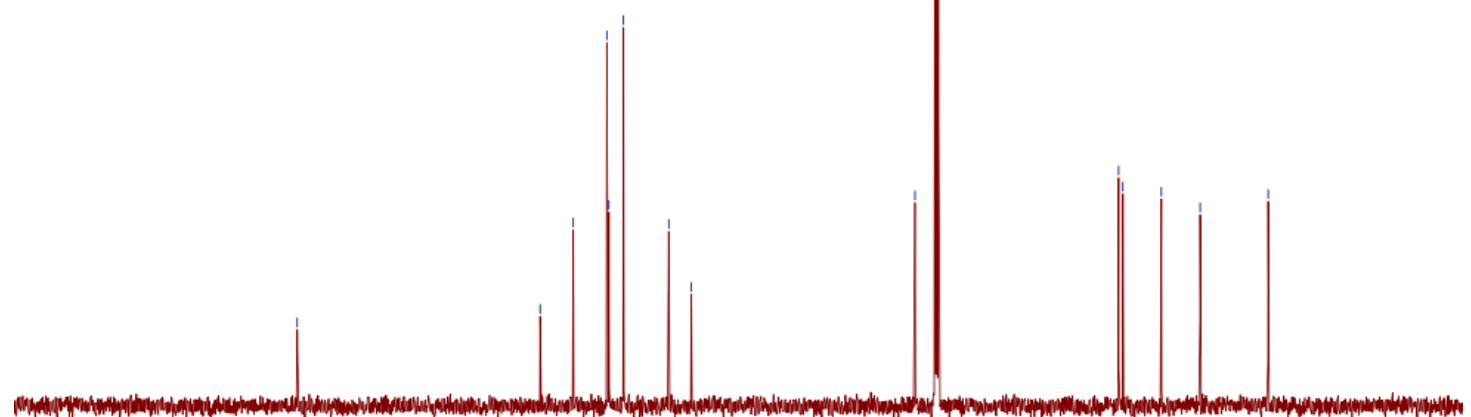

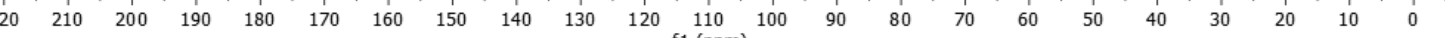




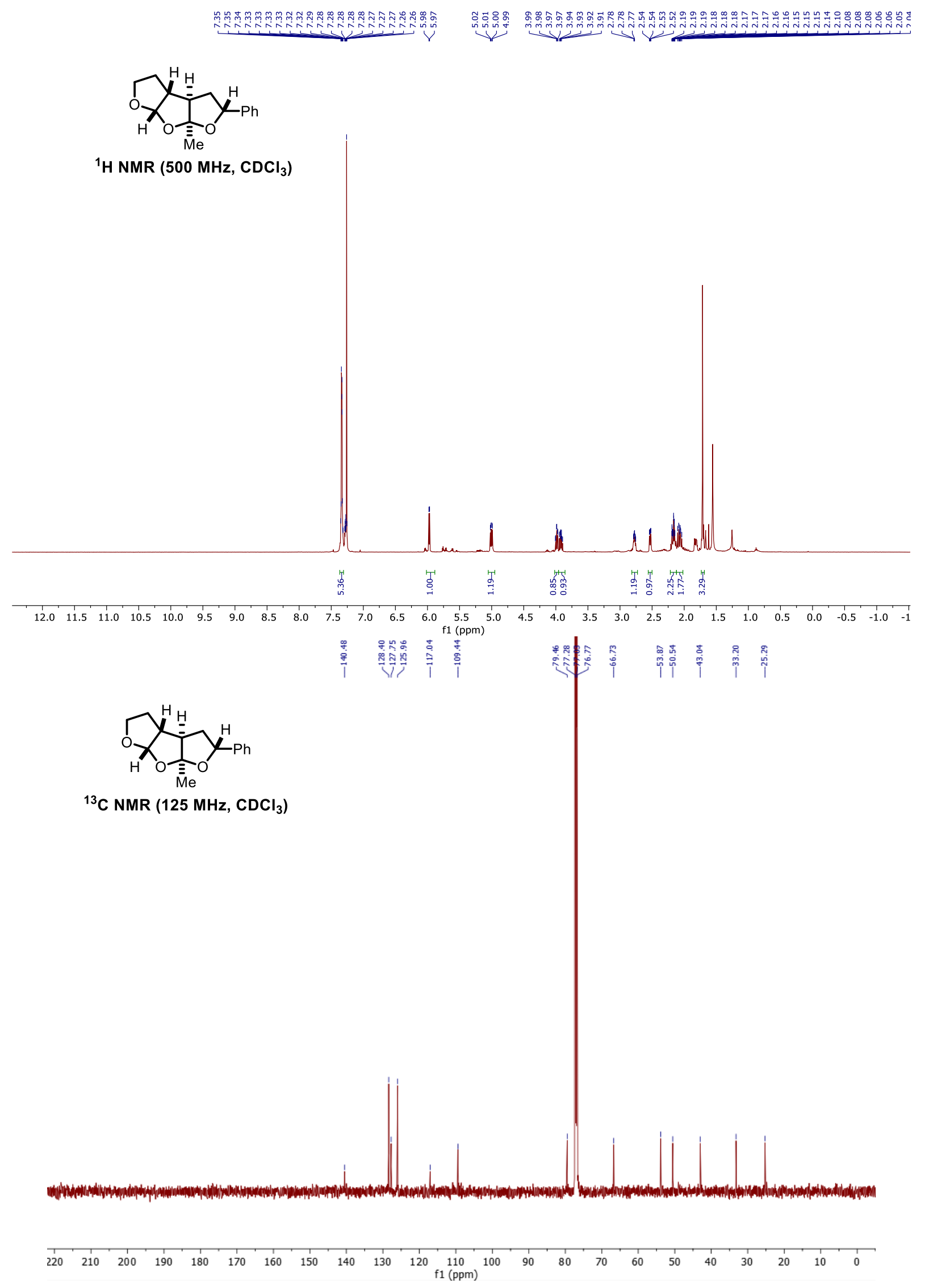




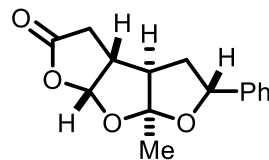

${ }^{1} \mathrm{H}$ NMR (500 MHz, $\left.\mathrm{CDCl}_{3}\right)$

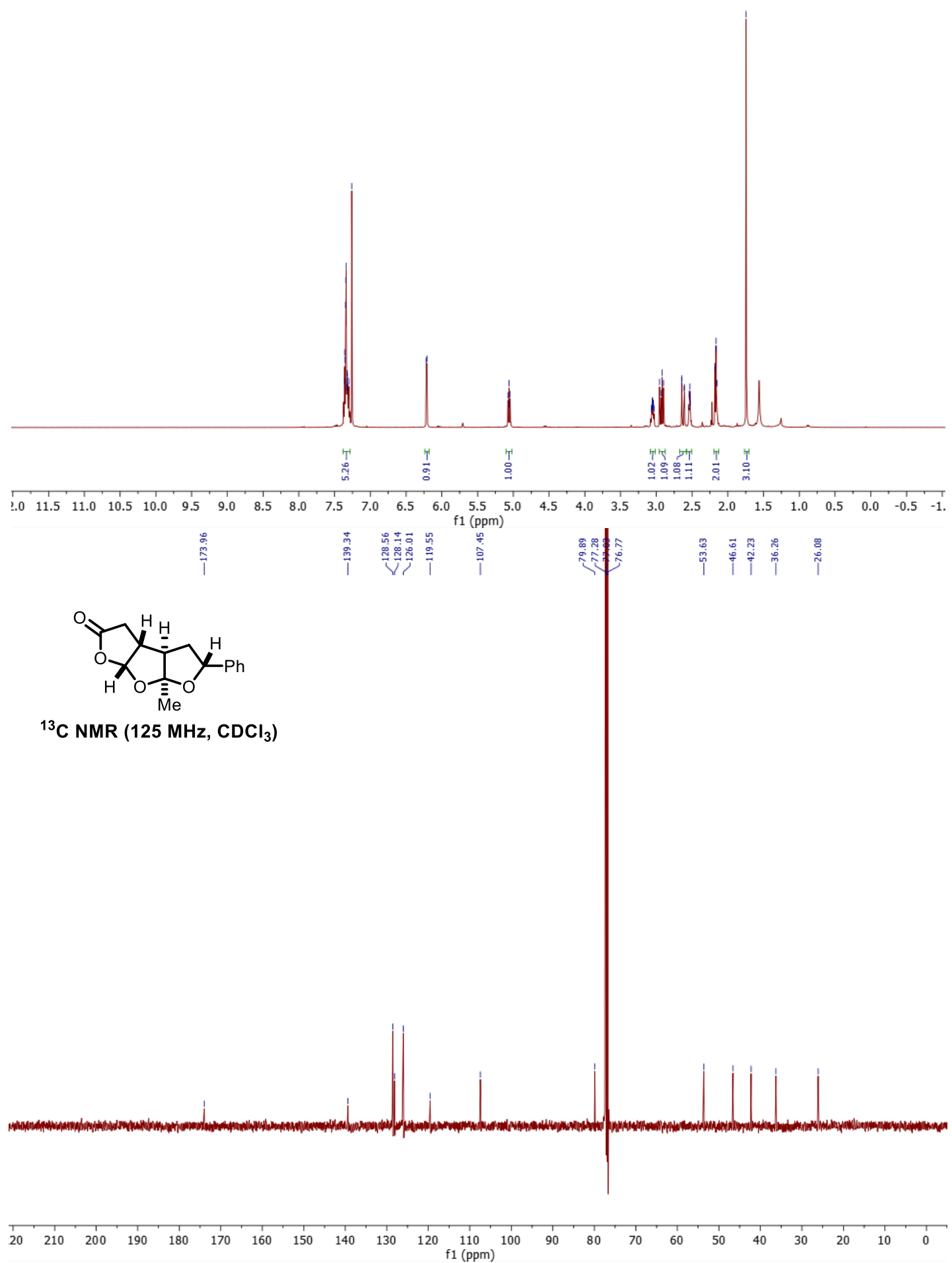

